Ameisen als Schlüsseltiere in einem Grasland

This work is licensed under the Creative Commons License 2.0 "by-nd", allowing you to download, distribute and print the document in a few copies for private or educational use, given that the document stays unchanged and the creator is mentioned. You are not allowed to sell copies of the free version.

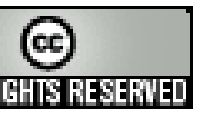


erschienen als Band 1 in der Reihe „Biodiversity and Ecology Series“des Göttingen Centre for Biodiversity and Ecology

Universitätsverlag Göttingen 2006 
Christian Platner

\section{Ameisen als Schlüsseltiere in einem Grasland}

Studien zu ihrer Bedeutung für die

Tiergemeinschaft, das Nahrungsnetz und das Ökosystem

Biodiversity and Ecology Series:

Band 1

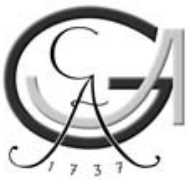

Universitätsverlag Göttingen

2006 


\section{Bibliographische Information der Deutschen Nationalbibliothek}

Die Deutsche Nationalbibliothek verzeichnet diese Publikation in der

Deutschen Nationalbibliographie; detaillierte bibliographische Daten sind im Internet über $<$ http://dnb.ddb.de> abrufbar.

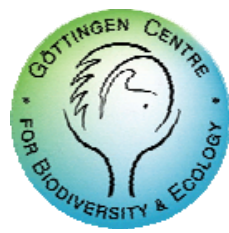

Editor PD Dr. Frank Thomas

Göttingen Centre for Biodiversity and Ecology,

Georg-August-Universität Göttingen, www.biodiversitaet.gwdg.de

Anschrift des Autors:

Dr. Christian Platner

Johann-Friedrich-Blumenbach-Institut für Zoologie und Anthropologie Abteilung Ökologie

Berliner Str. 28

37073 Göttingen

Dieses Buch ist auch als freie Onlineversion über die Homepage des Verlags sowie über den OPAC der Niedersächsischen Staats- und Universitätsbibliothek (http://www.sub.unigoettingen.de) erreichbar und darf gelesen, heruntergeladen sowie als Privatkopie ausgedruckt werden. Es ist nicht gestattet, Kopien oder gedruckte Fassungen der freien Onlineversion zu veräußern.

Titelbild: Mögliche Wechselwirkungen zwischen Mikroklima, Ameisen, Boden, Pflanzen und anderen Organismen (Maulwurf „Krtecek“ Zdenek Miler, Grafik Christian Platner)

Umschlagentwurf: Margo Bargheer

Dieses Buch wurde auf FSC-zertifiziertem Papier gedruckt.

FSC (Forest Stewardship Council) ist eine gemeinnützige und unabhängige

Organisation zur Förderung verantwortungsvoller Waldwirtschaft.

(C) 2006 Universitätsverlag Göttingen

ISSN -1863-3935

ISBN-10 3-938616-05-9

ISBN-13 978-3-938616-05-5 



\section{Inhalt}

1. Einleitung __ 1

2. Untersuchungsgebiet___ 7

2.1. Naturraum und Topographie ___ 7

2.2. Klima und Witterung___ 9

2.3. Geologie und Boden _ 10

2.4. Landnutzung und Vegetation___ 11

3. Methodik__ 17

3.1. Quantitative Erfassung der Bodenfauna___ 17

3.2. Bestimmung der Tiere und ihrer Biomasse___ 19

3.3. Chemische und bodenbiologische Analysen___ 19

3.4. Pflanzenwachstum und Mikrokosmen __ 20

3.5. ${ }^{15} \mathrm{~N}$ und ${ }^{13} \mathrm{C}$-Analysen ___ 20

3.6. Statistische Analysen___ 21

I. Umwelteinflüsse und Tiergemeinschaften

4. Verbreitungsmuster und Diversität von Ameisen, Spinnen und anderen Bodentieren in einem Gradienten vom Mischwald zum Trockenrasen auf Kalkgestein __ 23

4.1. Einleitung ___ 23

4.2. Methodik __ 25

Raster 25

Probenahme und Erfassung ergänzender Daten ___ 26

Mikroklimatische Messungen und Analyse der Baumkronenstruktur

Statistische Auswertungen __ 28

4.3. Ergebnisse___ 30

4.3.1. Biodiversität _ 30

Zikaden 30

Spinnen —31 
Ameisen

4.3.2. Verteilung der Tiergruppen in Abhängigkeit von der Umwelt 33

Wald-Wiese-Gradient __ 33

4.3.3. Räumliche Verteilung der Tiergruppen __ 39

4.3.4. Verteilung der Arten in Abhängigkeit von der Umwelt ___ 45

Pflanzen __ 45

Zikaden__ 46

Spinnen__ 46

Ameisen _ 47

4.4. Diskussion__ 50

4.4.1. Diversität der Ameisenfauna___ 50

Erfassungsmethoden $\quad 50$

Ameisen _ 50

4.4.2. Räumliche Beziehungen zur übrigen Tierwelt und zur Umwelt53

4.4.3. Interaktionen mit Saprophagen, Phytophagen und Zoophagen?

5. Einfluss des Mikroklimas: Auswirkungen experimenteller

Veränderungen auf die Bodenmakrofauna einer trockenen

Glatthaferwiese 57

5.1. Einleitung 57

5.2. Methodik 59

5.3. Ergebnisse 62

5.3.1. Auswirkungen auf den Boden 62

Abiotische Bedingungen__ 62

Mikroflora _ 65

5.3.2. Auswirkungen auf die Bodentiere___ 65

Mikrofauna: Nematoda___ 65

Mesofauna _ 65

Saprophage und Pantophage ___ 67

Phytophage und Pflanzen__ 69

Zoophage 71

5.3.3. Zeitliche Unterschiede in der Wirkung auf die

Bodentiergemeinschaft

5.3.4. Auswirkungen auf die Ameisengemeinschaft___ 76

5.4. Diskussion 77 


\section{Stellung und Funktion der Ameisen im Nahrungsnetz}

6. Nahrungsbiologie und Ermittlung der trophischen Ebene durch Analyse von stabilen Isotopen

6.1. Einleitung 81

6.2. Methodik 83

6.2.1. Nahrungsnetzanalyse 83

6.2.2. Laborversuch 84

6.3. Ergebnisse 86

6.3.1. Nahrungsnetzanalyse 86

Einordnung der Ameisen in das Nahrungsnetz 86

Vergleich verschiedener Ameisenarten und ihrer

Entwicklungsstadien 88

6.3.2. Laborversuch 90

Boden 90

Buschbohnen 91

Blattläuse 93

Ameisen 94

6.4. Diskussion 95

7. Interaktionen mit Spinnen und Phytophagen auf Halbtrockenrasen: Intragilden-Prädation, Konkurrenz und Trophobiose 99

7.1. Einleitung 99

7.2. Methodik 100

Versuchsaufbau 100

Beprobung 102

Auswertung 103

Ameisenausschluss auf Bäumen und Sträuchern 103

7.3. Ergebnisse 104

Ameisen 104

Spinnen 105

Reaktion anderer Arthropodengruppen 107

Geschlossene Parzellen 108

Ameisenausschluss auf Bäumen und Sträuchern 108

7.4. Diskussion 110 
III. Beeinflussung des Bodens und Wirkungen im Ökosystem

8. Biotische und abiotische Bodenveränderungen und deren bottomup Wirkung auf das Wachstum verschiedener Pflanzen 113

8.1. Einleitung 113

8.2. Methodik 114

Freilanduntersuchungen 114

Laborversuch 115

8.3. Ergebnisse 116

Bodenveränderungen 116

Auswirkungen auf andere Bodenbewohner 118 Laborversuch 120

8.4. Diskussion 121

9. Interaktionen mit der Bodenfauna und Effekte im unterirdischen

Nahrungsnetz 123

9.1. Einleitung 123

9.2. Methodik 124

9.3. Ergebnisse 126

9.4. Diskussion 132

10. Diskussion \& Schlussfolgerungen 135

11. Zusammenfassung 143

I. Umwelteinflüsse und Tiergemeinschaften 143

II. Stellung und Funktion der Ameisen im Nahrungsnetz 144 III. Beeinflussung des Bodens und Wirkungen im Ökosystem 145

12. Literatur 147

Danksagung 164 Anhang 166

A: Araneida 166

B: Trockengewicht einiger Spinnenarten 168

C: Zikadenarten auf Wiese 1 168

Lebenslauf 170 
Für ROSA, JOHANNA und PAULINA

Geb hin zur Ameise, du Fauler, sieb ihr Tun an, damit du klug werdest. Obgleich sie kein Oberhaupt, keinen Vorsteher und Gebieter hat, bereitet sie doch im Sommer ibr Brot, sammelt in der Erntezeit ibre Nabrung ein.

Sprüche 6, 6-8 



\section{Einleitung}

Ameisen gehören neben den Menschen weltweit zu den vorherrschenden Landorganismen und beeinflussen in besonderem Maße das Leben zahlloser anderer Pflanzen und Tiere (Hölldobler \& Wilson 1990). Vermutlich wird mehr als 50\% der weltweiten Insektenbiomasse von Ameisen und anderen sozialen Insekten gebildet (Hölldobler \& Wilson 1995). Ihre Sozialität ermöglicht eine effektive Nutzung vorhandener Ressourcen; durch Kooperation und Kommunikation können sie ihre Umwelt in hohem Maße kontrollieren und verändern (Brian 1983, Hölldobler \& Wilson 1990). So gehören Ameisen in vielen Habitaten zu den dominanten Arthropoden über und unter der Bodenoberfläche, wodurch sie eine zentrale Stellung in vielen biologischen Gemeinschaften einnehmen. Das Bindeglied zwischen der Gemeinschaft der Organismen und dem eher prozessorientierten Ökosystem-Modell wird durch ein Netzwerk aus Interaktionen und Stoffflüssen zwischen den einzelnen Bestandteilen gebildet. Ameisenkolonien haben im Vergleich zu vielen anderen Wirbellosen sehr hohe Metabolismusraten und Biomassezuwächse (Pêtal 1978), wodurch sie einen erheblichen Einfluss innerhalb dieses Nahrungsnetzes haben können (z.B. Letourneau \& Dyer 1998), und somit auch als Modellorganismen für die Untersuchung und das tiefere Verständnis von ökologischen Zusammenhängen sehr geeignet erscheinen.

Die Grundlage für ein biologisches Konzept eines Ökosystems bildet die Differenzierung in trophische Kompartimente, die über Stoffflüsse miteinander in Verbindung stehen (Schaefer 1996a); damit stellt das komplexe Gefüge der Nahrungsbeziehungen der entsprechenden Lebensgemeinschaft eine entscheidende 
Grundlage für die Stoffkreisläufe und Energieflüsse in ökologischen Systemen dar. Mechanismen für die Struktur solcher Nahrungsnetze werden heute intensiv diskutiert (z.B. Pimm et al. 1991, Polis 1994, 1998, Polis \& Winemiller 1996). Neben Produktivität (bottom-up-Wirkung) und Feinddruck (top-down-Effekt) spielen viele weitere Einflussgrößen wie indirekte Effekte oder nicht-trophische Interaktionen eine Rolle. Eine wichtige Funktion haben dabei sogenannte Schlüsselarten (keystone species, Paine 1969, Krebs 1985), durch deren Wegfallen sich das System stark verändern würde. Die entscheidenden Beziehungen dabei werden meist trophisch definiert. Für Organismen, die ihren Lebensraum gestalten, verändern und erhalten, führten Jones et al. (1994) den Begriff „Ecosystem Engineers“ ein; für Ameisen nennen sie als Beispiele für solches ecosystem engineering das Verändern der Bodenstruktur, das Schaffen von Mikrohabitaten und die Beeinflussung der Vegetation.

Ameisen haben ihrerseits aber auch recht hohe Ansprüche an ihre Umwelt, besonders an die Temperatur: die meisten Arten werden erst über $10^{\circ} \mathrm{C}$ aktiv, ihre Larven sind auf Temperaturen über $20^{\circ} \mathrm{C}$ angewiesen (Hölldobler \& Wilson 1990). In wärmeren Klimazonen lassen sich entsprechend eine größere Artenvielfalt und häufig ein sehr starker ökologischer Einfluss der Ameisen finden; doch auch in gemäßigtem Klima gehören sie, in erster Linie am Boden, zu den wichtigsten Zoophagen (z.B. Jeanne 1979); ihre Populationsdichte bleibt auch hier, trotz niedrigerer Artenzahl in geeigneten Biotopen auf sehr hohem Niveau (Pisarski 1978). Durch konstruktive Thermoregulation (Unter-Stein- und Hügelnester) und Trophobiosen (Symbiose mit Blattläusen und anderen Phytophagen, die eine gleichbleibend hohe Nahrungsversorgung sichern kann) eroberten sie auch in Mitteleuropa fast alle Ökosysteme und spielen in vielen eine bedeutende Rolle (z.B. Seifert 1996).

Ziel dieser Untersuchung ist es, über die Funktion von Ameisen als Schlüsseltierarten bzw. Ökosystem-Ingenieure und die zugrundeliegenden Bedingungen und Einflusswege detailliertere Aufschlüsse zu erhalten. Ameisen verändern nicht nur ihre Umwelt sondern sind selbst an diverse Umweltbedingungen gebunden, insbesondere an geeignetes Mikroklima, Nahrung und Nistmöglichkeiten. Durch die Kombination von Zoophagie und Trophobiose und den hohen Energieumsatz sollten Ameisen eine zentrale Stellung im Nahrungsnetz einnehmen. Ameisen können das Gefüge der Interaktionen im Ökosystem sowohl von der Basis her durch Modulation der Ressourcenverfügbarkeit ('bottom-up'-Wirkungen) als auch von der Spitze her durch Fraßdruck auf ihre Beutetiere ('top-down'-Kontrolle) beeinflussen. Forschungsansatz sind dabei zwei zentrale Hypothesen:

1. Klima und Konkurrenz stellen die wichtigsten verteilungsbestimmenden Faktoren für Ameisen dar. Bei ausreichender Konstanz geeigneter Umweltbedingungen besetzen Ameisen als soziale Insekten das „ökologische Zentrum“ und drängen solitäre Arten an die Peripherie.

2. Ameisen wirken über mindestens zwei grundsätzlich verschiedene Wege auf das Nahrungsnetz ein: als „soil engineers“ von der Basis her, wo insbesondere hügelbauende Arten die räumliche Vielfältigkeit und damit die Biodiversität för- 
dern, und als Prädatoren und über die Trophobiose von der Spitze her, wobei die omnivoren Ameisen direkt auf verschiedene trophische Ebenen zugreifen.

Die Überprüfung dieser Hypothesen auf den verschiedenen Ebenen des angesprochenen Ökosystemmodells ergibt folgende Dreiteilung dieser Arbeit:

I. Umwelteinflüsse und die Zusammensetzung der Tiergemeinschaft.

II. Stellung und Funktion der Ameisen im Nahrungsnetz.

III. Beeinflussung des Bodens durch Ameisen und ihre Wirkungen im Ökosystem.

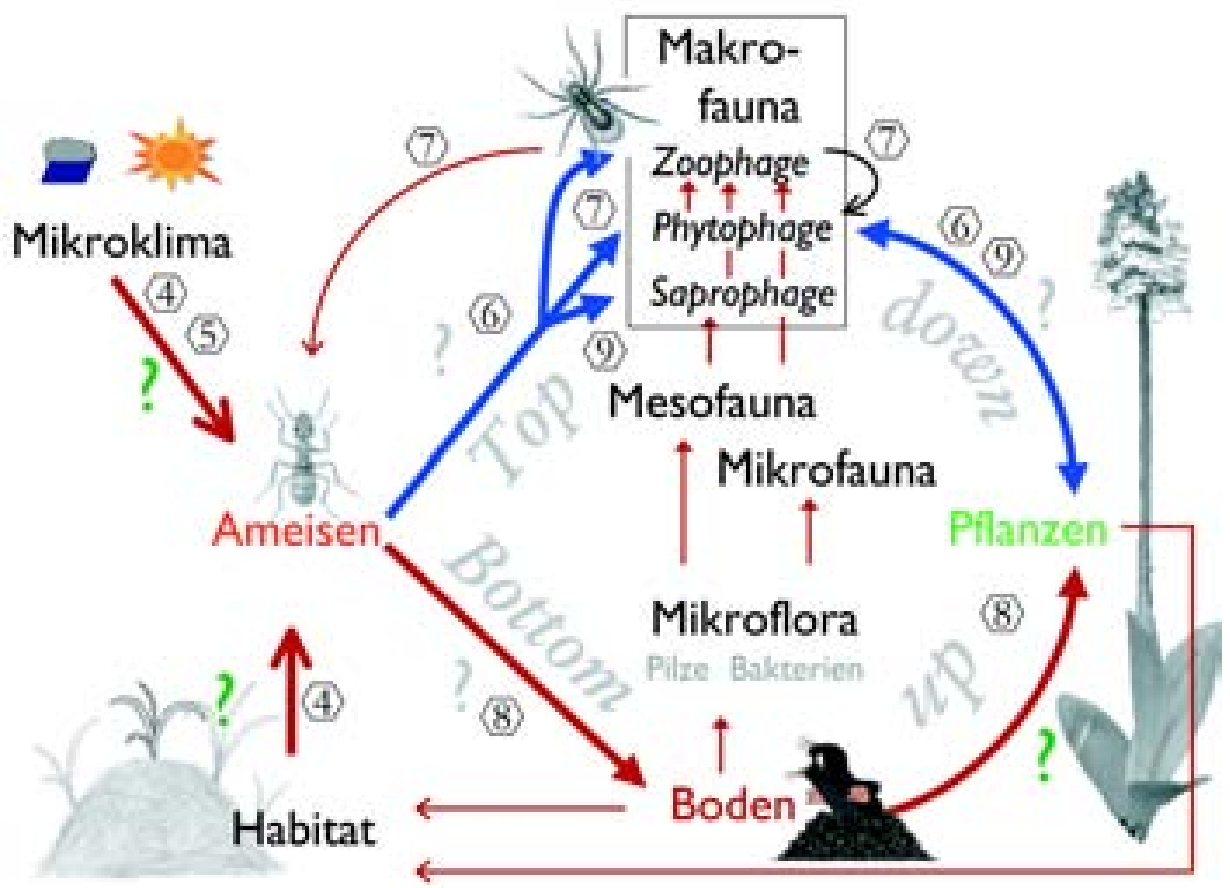

Abb. 1: Mögliche Wechselwirkungen zwischen Mikroklima, Ameisen, Boden, Pflanzen und anderen Organismen (Ziffern kennzeichnen die folgenden Kapitel, in denen die jeweiligen Effekte untersucht werden; Maulwurf-Zeichnung „Krtecek“"von Zdenek Miler)

Die Kenntnis der Diversität der Biozönose ist die Grundlage für die Beurteilung ihrer steuernden Wirkung auf Ökosystemfunktionen. Die Überprüfung und das tiefere Verständnis der aus Beobachtungen gewonnenen Erkenntnisse ist jedoch nur durch Experimente möglich.

Für jeden Teil der Fragestellung (vgl. Abb. 1) wurden deshalb deskriptive Untersuchungen zur Erfassung der Biodiversität mit Experimenten kombiniert, die zur Klärung folgender Fragen dienen sollten:

I. 1. Wie besiedeln Ameisen eine Fläche an der Schnittstelle verschiedener Lebensräume? Lassen sich Sukzessionsprozesse erkennen? Welche Korrelationen bestehen dabei mit der Dichte anderer Gruppen der Makrofauna und mit wichtigen Umweltfaktoren? 
I. 2. Welchen Einfluss haben einzelne Faktoren des (Mikro-)Klimas auf die verschiedenen Gilden der Bodentiergemeinschaft? Können klimatische Veränderungen die steuernde Wirkung auf Ökosystemprozesse verschieben?

II. 1. Welche relative Stellung nehmen verschiedene dominante Ameisenarten mit unterschiedlicher Lebens- und Ernährungsweise im Nahrungsnetz ein? Führt eine Veränderung des Nahrungsangebotes zu Verschiebungen in der Nutzung von Pflanzenläusen?

II. 2. Wie interagieren Ameisen mit anderen wichtigen Prädatoren? Bewirken sie eine top-down Kontrolle anderer trophischer Gruppen?

III. 1. Welche Funktion haben Ameisen als Regulatoren von Pflanzenwachstum über bottom-up Veränderungen in der Rhizosphäre?

III. 2. Haben Ameisen eine modulierende Wirkung auf das Pflanzenwachstum auch über top-down Effekte auf andere Bodentiere?

Auf einem Halbtrockenrasen wurde das Verteilungsmuster der Ameisenarten und weiterer wichtiger Bodentiere in einem Gradientengefüge verschiedener Sukzessionsstufen und kleinräumiger Habitatvariationen in Abhängigkeit von den jeweiligen Umweltbedingungen raumbezogen erfasst. Im Hinblick auf korrelative Beziehungen der Tiergruppen und gemeinschaftsstrukturierende Habitateigenschaften wurden diese Verteilungsmuster mit multivariaten Analyse- und Ordinationsverfahren ausgewertet (Kapitel 4).

Insbesondere auf Wiesen, auf denen ein erheblicher Anteil der Primärproduktion in den Aufbau unterirdischer Biomasse investiert wird, kommt der Bodenfauna eine entsprechend wichtige Rolle im Ökosystem zu (Spedding 1971, Curry 1994). In diesem Rahmen hat die quantitative Erfassung der epi- und endogäischen Makrofauna eine hohe Bedeutung. Für diese Untersuchung wurde in großem Maße die relativ aufwändige Hitzeextraktion der Tiere aus Bodensäulen mit der KEMPSON-Methode angewandt, da sie bei der ökologischen Untersuchung solcher kleinräumig extrem reichhaltig strukturierten Lebensräume, wie z.B. einer Brachwiese, im Vergleich zu anderen Methoden deutlich zuverlässigere Daten der Individuendichten für die Streu- und obere Bodenschicht liefert.

Besonders intensiv wurde der Einfluss des Mikroklimas als vermutlich wichtigster abiotischer Umweltfaktor auf die Ameisen und die Bodentiergemeinschaft durch experimentelle Manipulation der Faktoren Sonneneinstrahlung und Niederschlag auf einer benachbarten Brachwiese untersucht (Kapitel 5).

Die Funktion von Ameisen als Schlüsseltierarten im Nahrungsnetz wurde ebenfalls deskriptiv und experimentell untersucht. Eine vergleichende Einordnung verschiedener dominanter Ameisenarten in trophische Ebenen des Nahrungsnetzes eines Halbtrockenrasens war durch Analyse der natürlichen Gehalte der stabilen Isotope ${ }^{13} \mathrm{C}$ und ${ }^{15} \mathrm{~N}$ möglich. Zur Untersuchung der durch Kombination von Trophobiose und Prädation einzigartigen Nahrungsbiologie der Ameisen auch im Labor wurde ein Mikrokosmossystem entwickelt und getestet (Kapitel 6). 
Die zweite wichtige Prädatorengruppe im Grasland, die Spinnen, verwirklichen als solitäre Arten mit rein zoophager Ernährung eine von den sozialen Insekten grundsätzlich verschiedene Lebensstrategie. Die Interaktionen dieser beiden räuberischen Tiergruppen durch Intragilden-Prädation, Störung und Konkurrenz und die Auswirkungen beider Gilden auf die übrige Makrofauna, insbesondere auf Phytophage, wurden durch einen faktoriellen Ausschlussversuch im Freiland untersucht (Kapitel 7).

Die zentrale Stellung der Ameisen im Nahrungsnetz diente als Grundlage für die Untersuchung ihrer Funktion als Regulatoren von Pflanzenwachstum. Der bottom-up Einfluss von Ameisen durch Bodenveränderungen in der Rhizosphäre und die top-down Wirkungen durch direkte Interaktionen auf die Diversität der übrigen Bodenfauna und die Wuchsleistungen verschiedener Pflanzenarten wurden im Labor (Kapitel 8) und im Freiland (Kapitel 9) mit Mikrokosmosexperimenten genauer analysiert.

Die durchgeführten Untersuchungen und Experimente erlauben am Beispiel der Ameisen als einer Schlüsseltiergruppe ein tieferes Verständnis des Zusammenspiels multitrophischer und nicht-trophischer Interaktionen. 


\section{Untersuchungsgebiet}

\subsection{Naturraum und Topographie}

Die untersuchten Flächen liegen zwischen 180 und $200 \mathrm{~m}$ ü. NN auf einem Muschelkalkhang $3 \mathrm{~km}$ nordwestlich von Witzenhausen in Freudenthal (Abb. 2). Der Ortsteil liegt in einer typischen Mittelgebirgslandschaft im nordöstlichen Teil Hessens im Naturraum „Unteres Werratal“ zwischen Kaufunger Wald und Sandwald. Diese zwei bewaldeten Buntsandstein-Plateaus bilden mit Höhen von 400 bis 477 $\mathrm{m}$ ü. NN die natürliche Abgrenzung zum restlichen Osthessischen Bergland und dem Kasseler Becken im Süden und zum Sollingvorland und der Leine-Senke im Norden (und teilweise auch die Landesgrenze zu Niedersachsen). Die Wiesen 1 \& 2 liegen unmittelbar neben den Meridianen $9^{\circ} 50^{\prime}$ östlicher Länge von Greenwich und $51^{\circ} 22^{\prime}$ nördlicher Breite am südwestlichen Waldrand vor dem Großen Mittelberge; Wiese 3 liegt am südlichen Waldrand vor dem Kleinen Mittelberg. Beide Berge sind wie der benachbarte Badenstein Muschelkalkkuppen, die durch einen Grabenbruch, durch den heute auch die Werra fließt, unter das Niveau der umliegenden Buntsandsteinberge gesunken sind. 


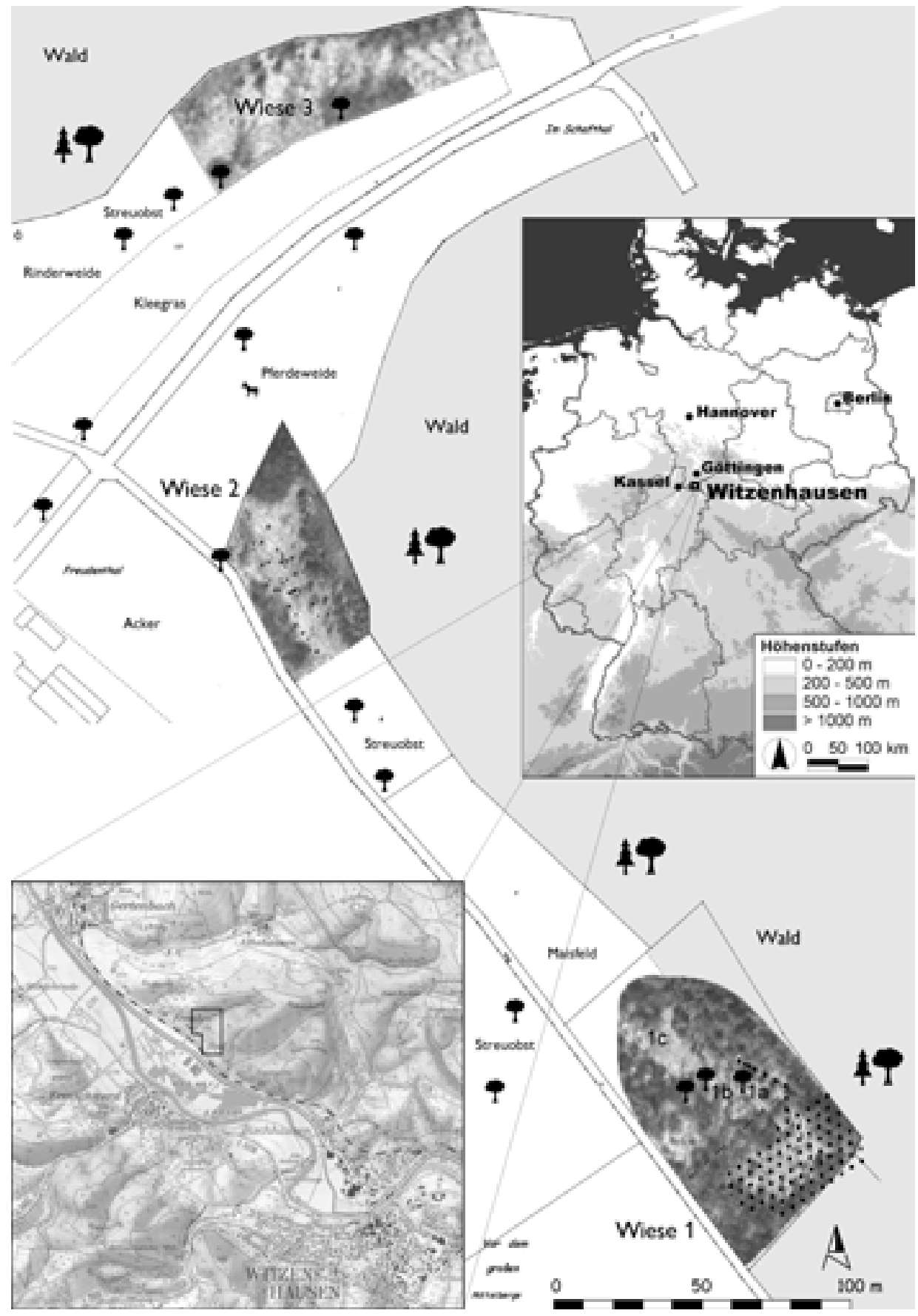

Abb 2: Lage der drei Versuchsflächen im Untersuchungsgebiet Witzenhausen-Freudenthal (Kartenund Luftbildausschnitte aus HVRKF 1999, 2003, Stadt Witzenhausen 2002, Sübrig 2004) 


\subsection{Klima und Witterung}

Das Witzenhäuser Werratal und die beiderseits begleitenden Höhen bis in ca. 350 m Höhe zeichnen sich bei mittleren Jahresniederschlägen von ca. $650 \mathrm{~mm}$ durch mildes, sommerwarmes Klima unter atlantischem Einfluss aus. In der HauptWachstumsperiode (Mai-Juli) betragen die mittleren Niederschläge $210 \mathrm{~mm}$ (Stein 1996).

Abgeschirmt durch die Höhenzüge des Kaufunger Waldes ist das untere Werratal relativ warm und trocken. Bei westlicher Hauptwindrichtung und mittleren Windgeschwindigkeiten von 3,2 bis $3,4 \mathrm{~m} / \mathrm{s}$ liegt die Jahresmitteltemperatur bei etwa $9{ }^{\circ} \mathrm{C}$ (im Januar $0^{\circ} \mathrm{C}$, im Juli $18{ }^{\circ} \mathrm{C}$ ). Insbesondere die zur Werra hin abfallenden Südhänge der Kalkkuppen liegen sehr wärmebegünstigt.

Pro Jahr erreichen jeden Quadratmeter etwa $975 \mathrm{kWh}$ als mittlere Jahressumme der Globalstrahlung. Die jährliche Verdunstung beträgt bei etwa 1500 Stunden Sonnenscheindauer durchschnittlich 575 l/m² (Stein 1996, HLUG 2003).

Während des Untersuchungszeitraums war die Witterung eher wärmer und feuchter als im langjährigen Mittel. Da der Betrieb der beiden nächstgelegenen Wetterstationen in Witzenhausen sowohl vom Deutschen Wetterdienst als auch von der Universität Kassel- Witzenhausen kurz vor bzw. nach Beginn meiner Untersuchungen eingestellt wurde, habe ich den Witterungsverlauf aus Tagesmittelwerten der Klimastation des Deutschen Wetterdienstes in Kassel (DWD 2003) berechnet und bei fehlenden Werten durch Messungen vom Versuchshof der Universität in Neu-Eichenberg-Hebenshausen (Meierbreite, 247 m, Fricke 2003) ergänzt (Abb. 3).

Die Klimadaten beider Stationen korrelierten in den Vorjahren sehr eng und hoch signifikant mit denen aus Witzenhausen. Die etwas höheren Niederschläge an der Station in Kassel erscheinen für das Untersuchungsgebiet in Freudenthal sowohl von der Meereshöhe als auch von der Lage am Westhang des Badensteins her eher repräsentativ zu sein; die Lufttemperaturen in Kassel korrelierten besonders gut und tagesgenau mit eigenen Messungen auf den Flächen, die nur über einen kürzeren Zeitraum möglich waren (Kapitel 4 \& 5). 


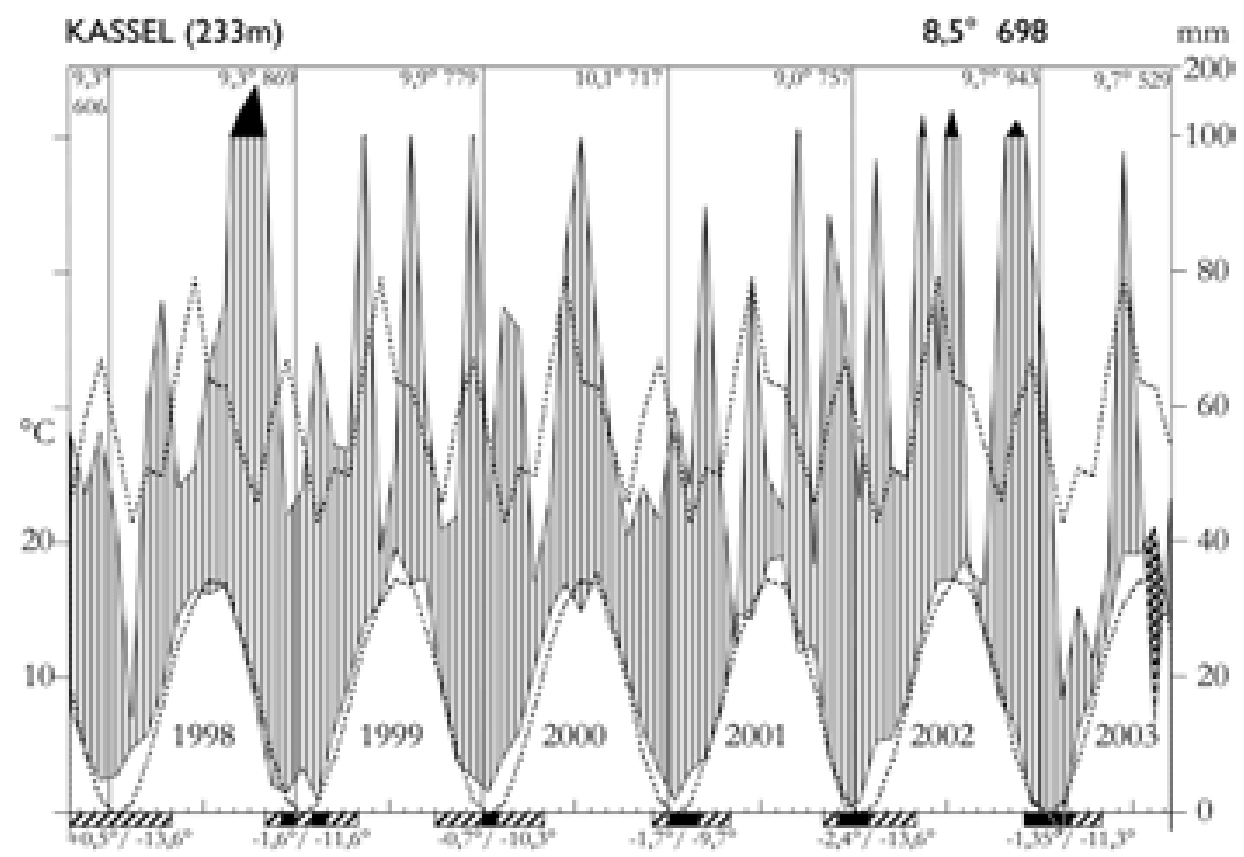

Abb. 3: Monatliche Mittelwerte der Lufttemperatur $\left[{ }^{\circ} \mathrm{C}\right]$ und Summen der Niederschläge [mm] von Oktober 1997 bis September 2003, DWD 2003, dargestellt als Klimadiagramm nach Walter (1990): vertikal schraffiert: relativ humide Jahreszeit, punktiert: Dürremonate, schwar:: perbumide Monate (Niederschlag > 100mm), gestrichelte Kurven: Normalwerte der Periode 1961-1990; über den Kurven für jedes Jahr Angabe der mittleren Jahrestemperatur und Niederschlagssumme, unter der Abszisse für jeden Winter das mittlere tägliche Minimum des kältesten Monats und das absolute Temperaturminimum (schwarzer Balken: kalte Jahreszeit, Monate mit mittl. Tagesmin. $<0^{\circ} \mathrm{C}$; schräg schraffierter Balken: Spät- oder Frühfröste, Monate mit abs. Min. $<0^{\circ} \mathrm{C}$ ).

\subsection{Geologie und Boden}

Das Ausgangsgestein der untersuchten Flächen und des oberhalb am Hang liegenden Waldes ist Unterer und Oberer Wellenkalk. Am flacher werdenden Hang unterhalb schließt sich bunter, lehmig-sandiger Mergel aus dem Oberen Buntsandstein an, der meist durch Löß und geschiebefreien Lehm aus dem Dilivium überlagert ist. In der Einsenkung zwischen Mittel- und Robelsberg („Schafthal“) liegt eine Deltabildung mit Schuttkegeln aus dem Alluvium.

Der Bodentyp ist eine Pararendzina (Abb. 4), die in den oberen Hangbereichen sehr flachgründig $(2-5 \mathrm{~cm})$ ausgebildet ist, an den unteren Hängen aber an Tiefe zunimmt. Dadurch sind auch deutliche Gradienten der Stickstoff-, Kohlenstoffund Kalkgehalte ausgeprägt. Der Gehalt an verfügbarem Stickstoff nimmt an allen drei Wiesen nach unten hin zu und beträgt in den oberen $5 \mathrm{~cm}$ des Mineral- 
bodens im Frühjahr durchschnittlich ca. $20 \mu \mathrm{g}$ N Ammonium und $12 \mu \mathrm{g}$ N Nitrat pro Gramm Trockengewicht. Im Sommer ist dieser Stickstoff fast komplett in der Vegetation gebunden. Durch den hohen Carbonatgehalt von durchschnittlich etwa $20 \%$ liegen auch die pH-Werte überall im neutralen Bereich zwischen 6,5 und 7,8. Genauere Analysen des Bodens auf allen drei untersuchten Wiesen sind im Ergebnisteil (Kapitel 8) dokumentiert.

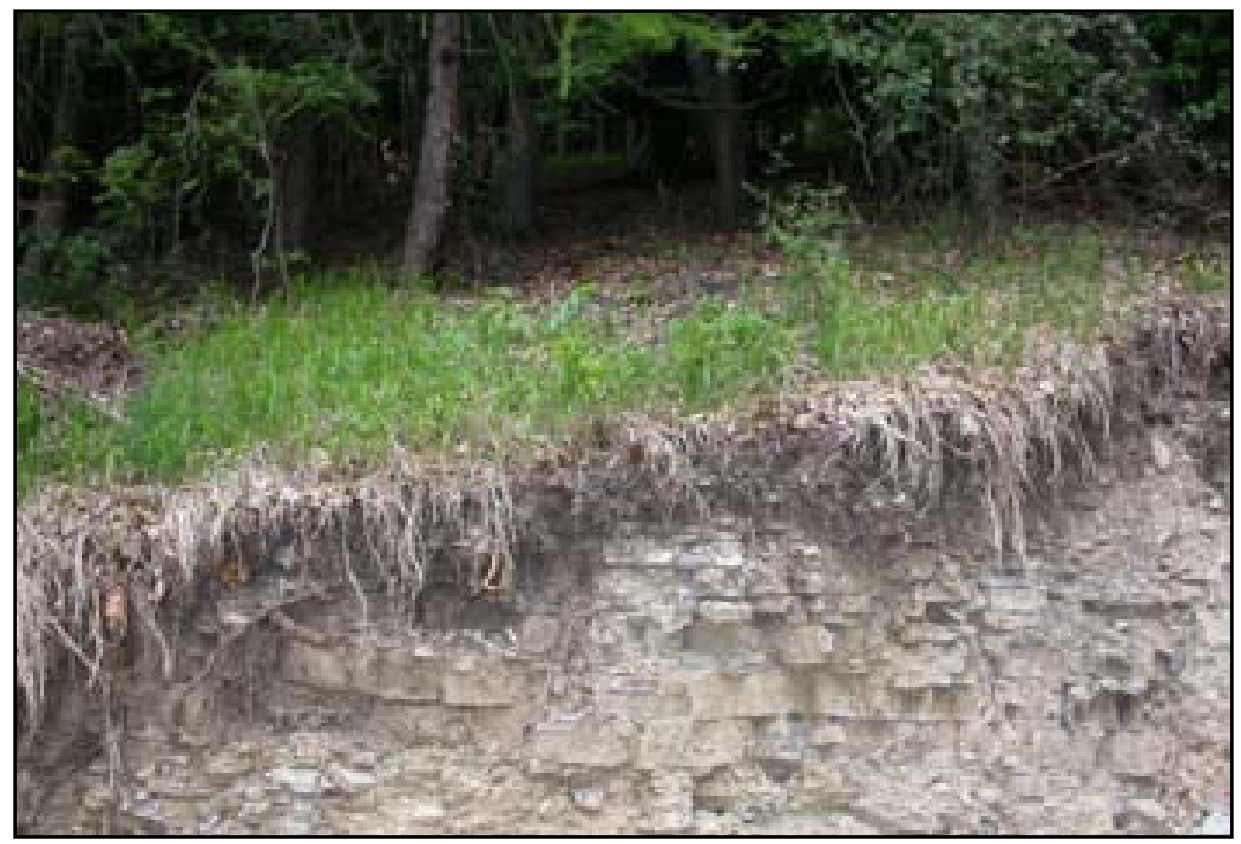

Abb. 4: Bodenprofil im Wald oberhalb von Wiese 1

\subsection{Landnutzung und Vegetation}

Der Badenstein ist aufgrund seiner besonderen Vegetation mit zahlreichen alten Eiben als flächenhaftes Naturdenkmal ausgewiesen. Durch Bahnlinie und Bundesstraße vom Untersuchungsgebiet getrennt, liegt am Werraufer das Naturschutzgebiet Freudenthal mit einigen Feuchtbiotopen; westlich vom Ortsteil Ermschwerd am gegenüberliegenden Werraufer „Auf dem Heegen“ finden wir einen ebenfalls unter Naturschutz stehenden Trockenrasen auf Muschelkalk.

Das Untersuchungsgebiet ist wie der ganze Naturraum historisch durch Weinund später Kirschanbau geprägt. Die bis vor etwa fünfzehn Jahren als Rinderweiden genutzten ehemaligen Streuobstwiesen an den Waldrändern liegen heute brach und bilden im unteren Hangbereich typische trockene Glatthaferwiesen, die nach oben zum Waldrand hin teilweise in Kalk-Halbtrockenrasen übergehen. Alle drei untersuchten Wiesen werden am Oberhang von einem Buchen-Kiefern- 
Mischwald mit eingestreuten Eiben und Lärchen begrenzt (Abb. 5). Der Wald gehört zum Schloss Berlepsch, wird im Auftrag des Grafen beförstert und ist komplett als Teil des FFH-Gebiets Werra- und Wehretal ausgewiesen (HLUG 2003).

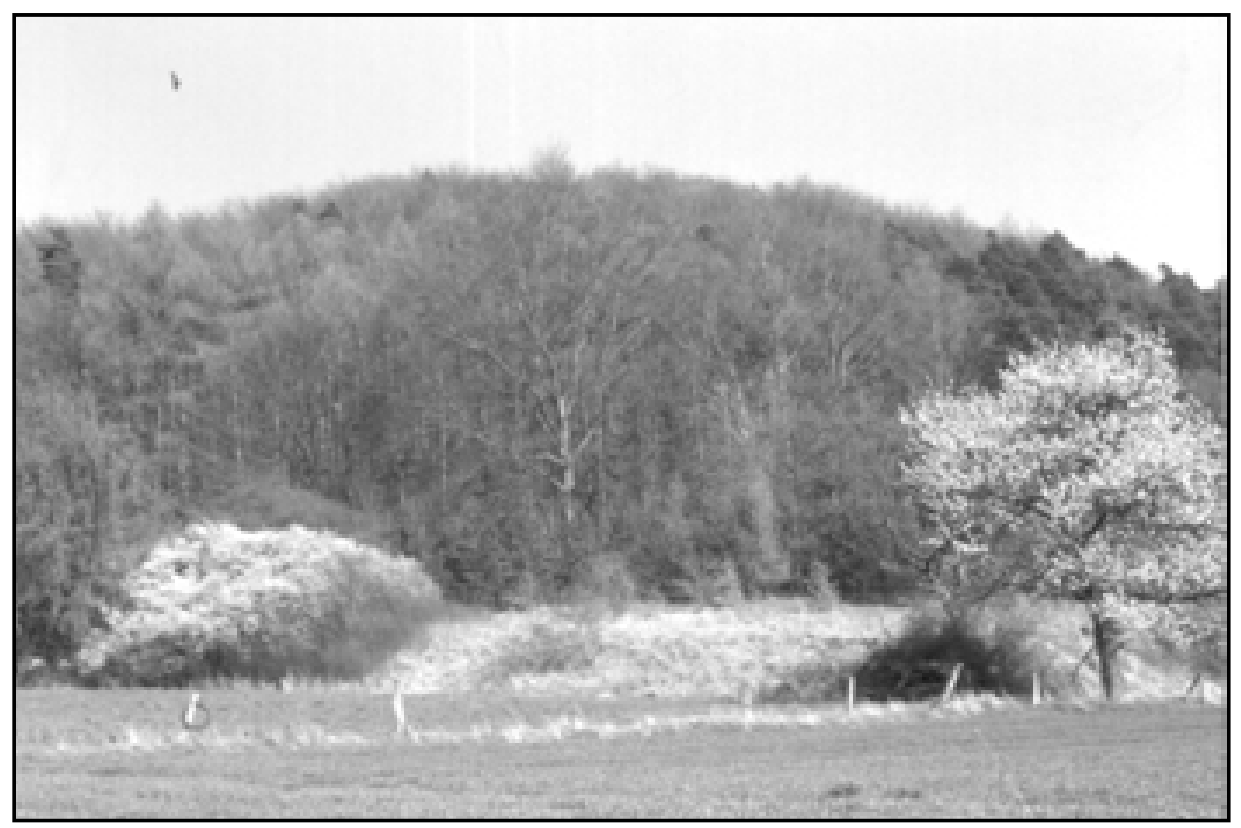

Abb. 5: Waldrand oberhalb des Brachwiesengürtels (Wiese 2 von Westen am 2. 4. 2002)

Wiese 1 grenzt im Nord- und Südosten an den Wald; an den Grundstücksgrenzen sind noch alte Kalksteinwälle erkennbar. Der nordöstliche Waldrand wird meist direkt von den Ästen der Buchen und Kiefern gebildet. Am südöstlichen Rand bildet eine Reihe Haselnuss-Sträucher die ehemalige Abgrenzung zum Wald; mittlerweile ist kräftiger Kiefern- und Hartriegel-Jungwuchs (bis etwa $10 \mathrm{~m}$ hoch) vorgelagert, so dass ein vielfältiger, fließender Übergang von der verbliebenen Offenfläche über verschiedene Gebüsche in den Wald entstanden ist.

Die Offenfläche ist im oberen Bereich etwa zur Hälfte ein artenreicher subozeanischer Kalk-Halbtrockenrasen (Mesobromion) mit eingestreuten Wacholderund Rosensträuchern und einem kleinen Bestand an Purpurknabenkraut. Die untere Hälfte ist eine teilweise schon stärker verbuschte, trockene Glatthaferwiese mit vielen Erdhügelnestern der Gelben Wiesenameise (Tab. 1).

Südwestlich am Hangfuß bildet ein Hain aus Hartriegel, Weißdorn, Feldahorn und alten Kirschen die Grenze zum Weg und den jenseits liegenden noch bewirtschafteten Äckern und Obstwiesen. Wiese 1 wird im Nordwesten durch ein dichtes Schlehe-Weißdorn-Gebüsch von der benachbarten Streuobstwiese 1b getrennt. 


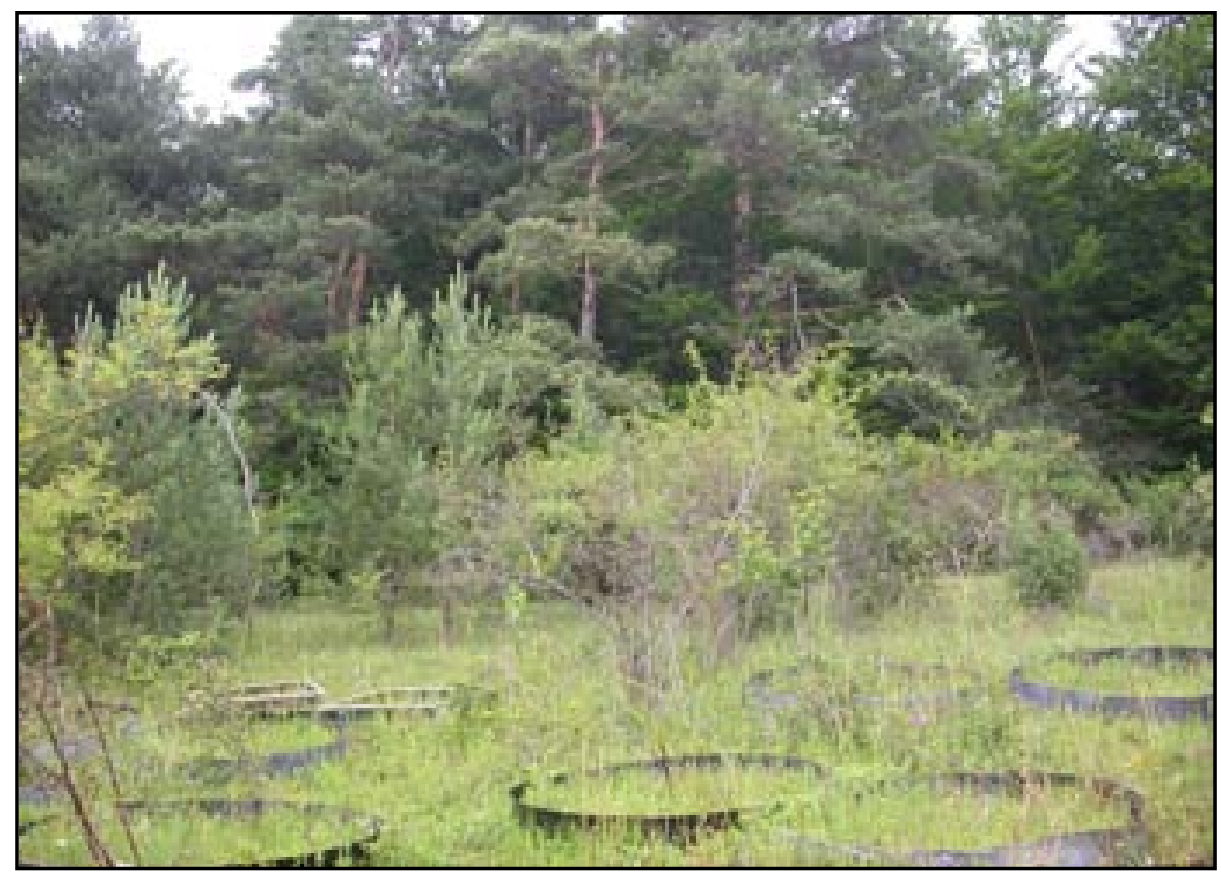

Abb. 6: Oberer Teil von Wiese 1 mit nordöstlich angrenzendem Rotbuchen-Waldkiefern-Bestand; vorne sind einige Parzellen des Prädatoren-Ausschluss-Versuchs erkennbar (28. 06. 2003).

Tabelle 1: Liste der Pflanzenarten auf Wiese 1, sortiert nach funktionellen Gruppen und den Werten der 1. Achse einer Hauptkomponentenanalyse für die Hangabschnitte 1-4 des Halbtrockenrasen-Glatthaferwiesen-Gradienten (vgl. Kap. 4.3.4; 1-5: Blöcke im Ausschlussversuch (Kap. 7), mittlerer Deckungsgrad in \% (Vegetationsaufnahme vom 5.7.2003, Sanders 2004) bzw. mittlere Biomassen aus je 5 Kempsonproben; O: Oberhang \& Waldrand, U: Unterhang \& Hain).

\begin{tabular}{|c|c|c|c|c|c|c|c|}
\hline Pflanzenart & $\backslash$ & Hangabschnitt $\mathrm{O}$ & 1 & 2 & & & $5 \quad \mathrm{U}$ \\
\hline
\end{tabular}

Gräser u.a. Monocotyledonae

Orchis purpurea W. Hudson 1762

$\mathrm{X}$

Elymus (=Agrypyron) repens (Linnaeus) Gould 1947

Poa pratensis Linnaeus 1753

Allium spec. Linnaeus 1753

Trisetum flavescens (Linnaeus) Palisot de Beauvois 1812

Bromus erectus W. Hudson 1762

Arrhenatherus elatius (Linnaeus) J. S. Presl \& C. B. Presl 1819

Brachipodium pinnatum (Linnaeus) Palisot de Beauvois 1812

$\begin{array}{cccccc}2 & & & & \\ 7 & 2 & 2 & & 4 & \\ 1 & & 2 & & 4 & \\ 1 & 3 & 4 & 2 & 2 & \\ 2 & & & 5 & & \\ & 2 & 5 & 5 & 2 & \\ & 1 & 5 & 30 & & \\ & & & & & \mathrm{X} \\ 152 & 127 & 115 & 188 & 146 & \\ 27 & 44 & 32 & 104 & 97 & \\ \end{array}$

Helictotrichon (=Avena) pubescens (W. Hudson) Pilger 1938

Biomasse Gräser 3. Mai $1998\left[\mathrm{~g} / \mathrm{m}^{2}\right]$

Biomasse Gräser 9. September $2003\left[\mathrm{~g} / \mathrm{m}^{2}\right]$ 
Tab. 1 - Fortsetzung

Pflanzenart

$\begin{array}{lllllll}\text { Hangabschnitt } \mathrm{O} & 1 & 2 & 3 & 4 & 5 & \mathrm{U}\end{array}$

\section{Kräuter}

Primula veris Linnaeus 1753

Thymus pulegoides Linnaeus 1753

Sanguisorba minor Scopoli 1772

Cirsium arvensis (Linnaeus) Scopoli 1772

Fragaria viridis Weston 1771

Bupleurum falcatum Linnaeus 1753

Euphorbia cyparissias Linnaeus 1753

Silene vulgaris (Moench) Garcke 1869

Knautia arvensis (Linnaeus) T. Coulter 1823

Leucanthemun vulgare Monnet de Lamarck 1779

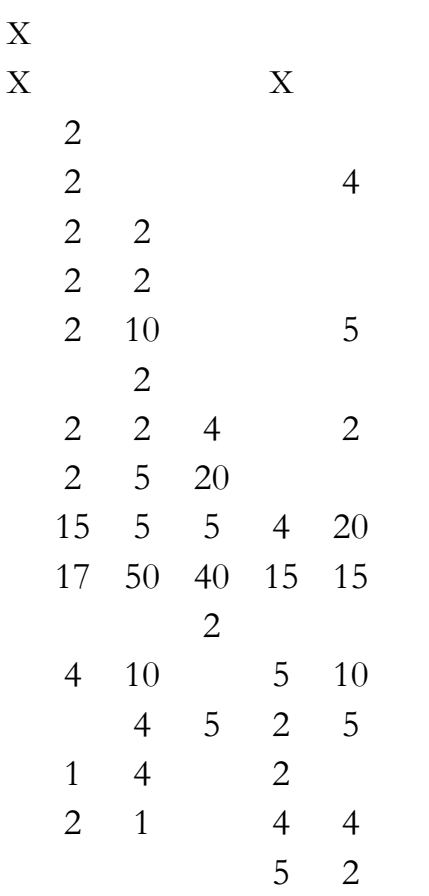

Agrimonia eupatoria Linnaeus 1753

Clinopodium vulgare Linnaeus 1753

Tanacetum vulgaris Linnaeus 1753

Viola hirta Linnaeus 1753

Anthriscus sylvestris (Linnaeus) G. F. Hoffmann 1814

Centaurea jacea Linnaeus 1753

Hypericum perforatum Linnaeus 1753

Galium album P. Miller 1768

\section{ren}

\section{Leguminosen}

Lotus corniculatus Linnaeus 1753

Medicago lupulina Linnaeus 1753

Astragalus spec. Linnaeus 1753

$\begin{array}{lll}6 & 1 & 5\end{array}$

$\begin{array}{lllll}10 & 4 & 10 & 20 & 10\end{array}$

Biomasse Kräuter 3. Mai 1998 [g/m²]

$\begin{array}{lllll}9,6 & 10, & 9,7 & 6,5 & 9,3\end{array}$

Biomasse Kräuter 9. September 2003 [g/m²]

$\begin{array}{lllll}64 & 86 & 89 & 88 & 118\end{array}$

Pflanzendichte (Deckung *Höhe [m] ges. Krautschicht)

$\begin{array}{lllll}42 & 85 & 102 & 114 & 102\end{array}$

Streuanteil

Biomasse Moose 3.Mai 1998 [g/m²]

\begin{tabular}{ccccc}
0 & 10 & 50 & 60 & 60 \\
\hline 135 & 146 & 119 & 36 & 28
\end{tabular}

Biomasse Moose 9. September $2003\left[\mathrm{~g} / \mathrm{m}^{2}\right]$

211173181150205

Deckungsgrad Moose

$\begin{array}{lllll}10 & 60 & 70 & 40 & 60\end{array}$

Anteil blanker Boden

$\begin{array}{lllll}30 & 0 & 5 & 0 & 0\end{array}$

\section{Gehölze}

Taxus baccata Linnaeus 1753 \& Larix decidua P. Miller 1768 X

Fagus sylvatica Linnaeus 1753

Juniperus communis Linnaeus 1753

Pinus sylvestris Linnaeus 1753

$\mathrm{X}$ 
Tab. 1 - Fortsetzung

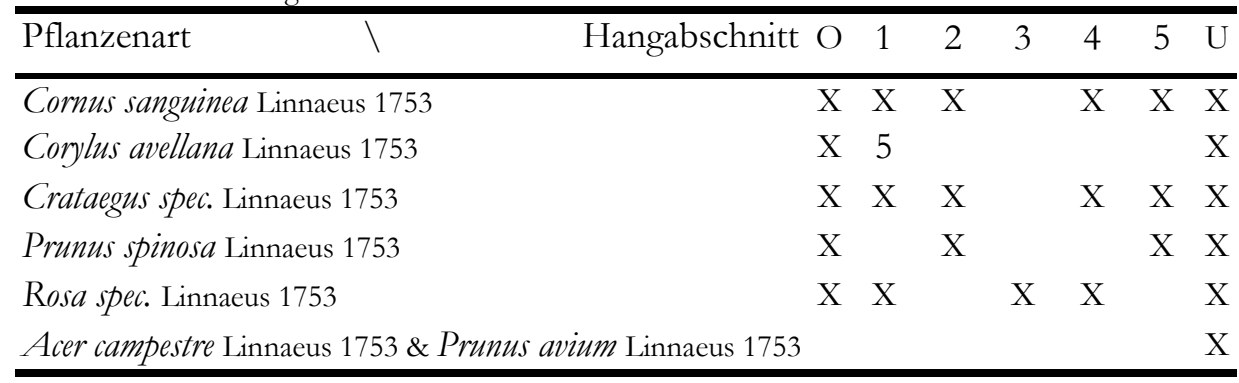

Auf Wiese 1 wurde die Rasteruntersuchung des Wald-Wiese-Gradienten (Kapitel 4), die Nahrungsnetzanalyse (Kapitel 6), das Prädatoren-Ausschluss-experiment (Abb. 6; Kapitel 7) und im unteren Bereich ein Teil der Nestboden-untersuchungen und des Freiland-Mikrokosmenversuchs (Kapitel 8 \& 9) durchgeführt.

Zwischen Wald und Weg reihen sich in nordwestlicher Richtung verschiedene Brachflächen aneinander; die der Wiese 1 benachbarte Fläche lässt sich in die Teilflächen 1a am Waldrand, 1b im unteren brachliegenden Streuobstbereich und 1c auf einem schmaleren Wiesenstreifen an einem sonnigen Kiefernjungwuchsrand unterteilen (Abb. 2). Diese kleineren Teilflächen wurden bei den Nahrungsnetzstudien (Kapitel 6 \& 7) ergänzend zu Wiese 1 untersucht, da sie bei ansonsten sehr ähnlichen Klima-, Vegetations- und Bodenbedingungen durch den Bestand an alten Kirschbäumen und zwei Kolonien von Formica pratensis Retzius interessante Vergleiche ermöglichten. Am Unterhang von Fläche 1c grenzt eine Wildfütterungstelle an, nordwestlich davon ein Wildacker, der seit etwa 15 Jahren durchgängig mit Mais und Erbsen bepflanzt wurde (Abb. 2: „Maisfeld“).

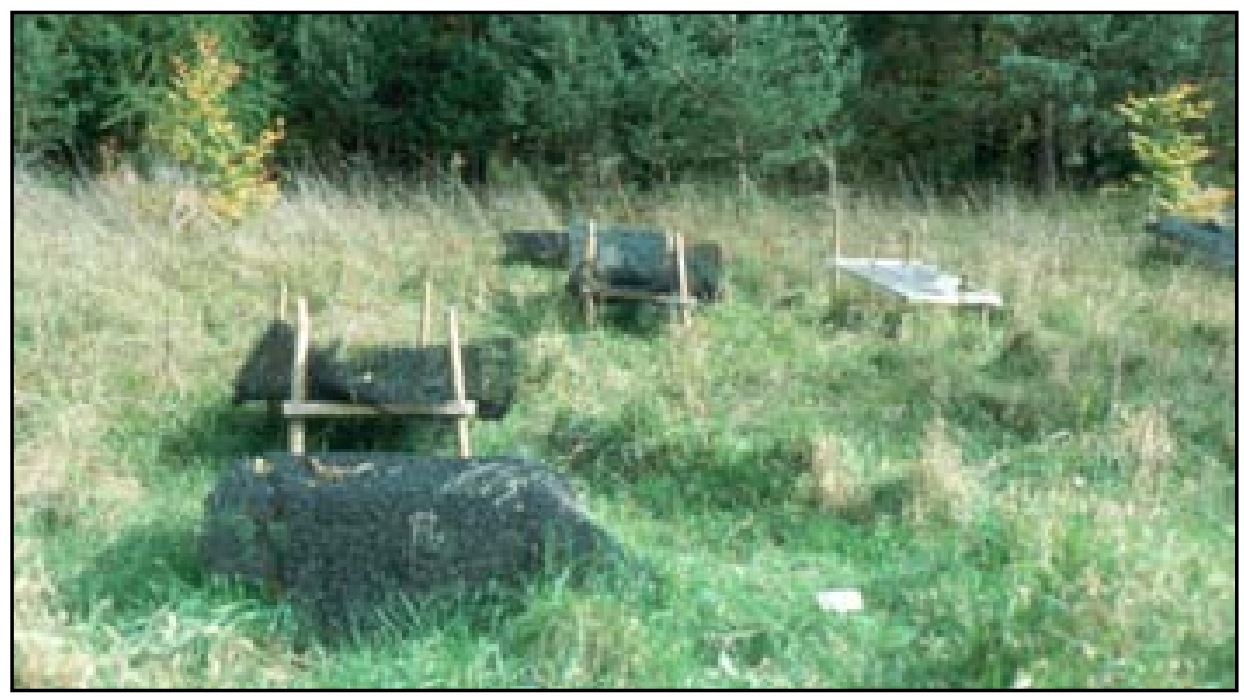

Abb. 7: Wiese 2 mit einigen Versuchs-Parzellen der Mikroklimamanipulation; im Hintergrund der Waldrand mit vordringendem Kiefern- und Buchen-Jungwuchs (18. 10. 1998). 
Durch eine weitere Streuobstwiese vom Maisfeld getrennt, bildet Wiese 2 den Abschluss der brachliegenden südwest-exponierten Hang-Grundstücke. Der Großteil der Fläche ist eine blumenreiche trockene Glatthaferwiese mit einer hohen Nestdichte der Gelben Wiesenameise Lasius flavus (F.), die hier die Einrichtung einer genügend großen Anzahl Versuchsparzellen zur Mikroklima-Manipulation ermöglichte (Abb. 7; Kapitel 5). Durch fehlende Mahd und Beweidung breitet sich auch hier der Jungwuchs der Waldbäume langsam auf die Wiese aus. Der Trockenrasen-Charakter am Oberhang vorm Waldrand ist deutlich schwächer ausgeprägt als auf Wiese 1. Am Unterhang dominiert teilweise Flaumhafer die Wiese, die hier durch eine Böschung und teilweise eine Hecke aus Esche, Kirsche und Weißdorn vom Weg und einer benachbarten Weide getrennt wird.

Jenseits einer kleinen Senke, die den Anfang des „Schafthals“ bildet, liegt am Hang des Kleinen Mittelbergs die Wiese 3. Der untersuchte Abschnitt ist südostexponiert, weniger flachgründig und hat einen deutlich niedrigeren Kalkgehalt als die beiden anderen untersuchten Wiesen. Teilweise stehen hier noch hochstämmigen Süß-Kirschbäume (Abb. 8). Ein vom Glatthafer dominierter Bereich diente bei der Nestbodenuntersuchung und dem Freiland-Mikrokosmenversuch neben den unteren Hangbereichen der Wiesen 1 \& 2 als dritte Vergleichsfläche. Die östlich und westlich davon gelegenen Bereiche sind stärker geneigt und haben deutlichen Trockenrasencharakter. Die zum Werratal exponierten angrenzenden Hänge bilden artenreiche aber stark verbuschte Trockenrasen aus. Ein Jahr nach Abschluss meiner Untersuchungen wurde die Wiese 3 nach mehrjähriger Pause wieder von Jungrindern beweidet (Abb. 61, S. 140).

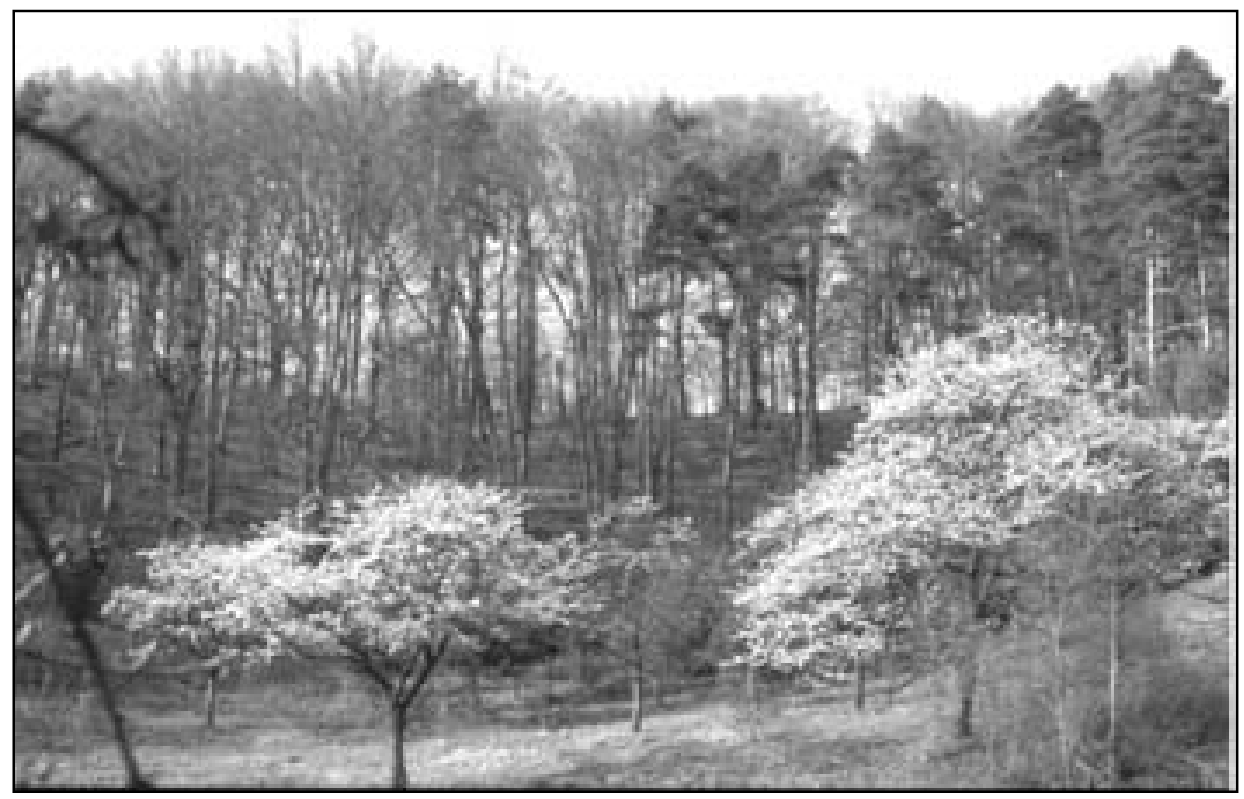

Abb. 8: Der flachere Bereich der nördlich im Untersuchungsgebiet gelegenen Wiese 3 diente bei den bodenbiologischen Untersuchungen und Experimenten als Vergleichsfläche (22. 04. 2002). 


\section{Methodik}

In diesem Kapitel werden nur versuchsübergreifende Methoden beschrieben; die Erläuterungen der einzelnen Versuchsanlagen und jeweiligen experimentellen Details folgen direkt in den jeweiligen Kapiteln.

\subsection{Quantitative Erfassung der Bodenfauna}

Die Makro- und Mesofauna wurde aus Bodenproben mit einer von Schauermann (1982) modifizierten Kempson- bzw. MacFadyen-Apparatur (MacFadyen 1961, Kempson et al. 1963), die Nematoden mit einer O'Connor-Naßextraktionsapparatur ausgetrieben (Heitkamp \& Schauermann 1982, Alphei 1995). Die Probenahme erfolgte mit Stechzylindern von 21 oder $5 \mathrm{~cm}$ Durchmesser $(\approx 1 / 28 \mathrm{bzw}$. $1 / 509 \mathrm{~m}^{2}$ ), die bis etwa $10 \mathrm{~cm}$ Tiefe eingestochen wurden. Aus den $21 \mathrm{~cm} \varnothing$ Bohrkernen wurde die Streuschicht gemeinsam mit den oberen $5 \mathrm{~cm}$ des Mineralbodens entnommen, so dass der Gras- und Wurzelfilz nicht getrennt werden mussten. Die $5 \mathrm{~cm} \varnothing$ Bohrkerne wurden in drei Horizonte gegliedert: Vegetation \& Streuauflage, 0-5 cm und 5-10 cm Bodentiefe; die Proben aus den Ameisennestern hatten praktisch keine Streuauflage, so dass diese Proben nur in zwei Horizonte geteilt wurden.

Das zeitliche Regime des Temperaturgradienten in der Kempson-Apparatur wurde in Anpassung an die Bedingungen des Trockenrasens gegenüber den Empfehlungen für Waldböden von Schauermann (1982) etwas beschleunigt; schon 
während der ersten 144 Stunden der Extraktion wurde der Gradient um täglich 5 ${ }^{\circ} \mathrm{C}$ erhöht. Dieser immer noch relativ langsam ansteigende Temperaturgradient gibt einerseits auch langsamen Bodentieren die Gelegenheit, die Bodensäule in Richtung der kühl-feuchten Luft über dem Auffangbehälter zu verlassen, lässt aber andererseits den wärmeliebenderen Ameisen auch genügend Zeit, den Boden kräftig umzugraben, was insbesondere bei Proben aus Nesthügeln mit lockerem Substrat und vielen Arbeiterinnen zu erheblichen Verunreinigungen der Probe führte.

Für die Nematodenerfassung wurden jeweils die oberen $5 \mathrm{~cm}$ der Bodensäulen aus drei Einstichen mit dem $5 \mathrm{~cm} \varnothing$ Bohrer im Labor gesiebt (4 mm) und zu einer Mischprobe vereinigt, um die Probenvarianz zu verringern. Von dieser Mischprobe wurden aus $15 \mathrm{~g}$ Frischgewicht mit einer Trichterextraktion nach dem Baermann-Prinzip die Nematoden in der O'Connor-Apparatur ausgetrieben.

Die Erfassung der epigäischen Fauna erfolgte zusätzlich quantitativ durch Absaugen definierter Flächen $\left(1 / 28 \mathrm{~m}^{2}\right.$ - Aussaugen eines zuvor aufgesetzten Stechzylinders) mit einem modifizierten Laub-Sauger (Stihl SH 85, Stihl AG, Waiblingen; 0,8 kW; $625 \mathrm{~m}^{3}$ Luftdurchsatz/h; Käschernetz im $10 \mathrm{~cm} \varnothing$ Saugrohr) und qualitativ durch Beobachtungen, Hand- und Käscherfänge und Bodenfallen.

Die extrahierten Tiere wurden, wie die Tiere aus den Bodenfallen oder den Handaufsammlungen, zur weiteren Aufbewahrung entweder aus der jeweiligen Fixierlösung in $70 \%$ iges Ethanol überführt oder, um die Gehalte der stabilen Kohlenstoff- und Stickstoff-Isotope unverändert zu lassen, direkt bei $-24{ }^{\circ} \mathrm{C}$ tiefgefroren (Tab. 2). Vor der Auszählung der Mesofauna wurden stark verschmutzte Proben mit Heptan gespült: die hydrophoben Oberflächen der Tiere bewirken ein Auftreiben bis zur Unterkante der organischen Phase; mineralische Partikel bleiben am Boden der wässrigen Phase. Nach Dekantieren wurden die Tiere wieder in Alkohol überführt.

Tabelle 2: Fixier- und Aufbewahrungslösungen bei den angewandten Methoden (Fixierlösungen mit etwas parfumfreiem Detergenz zur Verringerung der Oberflächenspannung)

\begin{tabular}{|c|c|c|}
\hline Methode & Fixierung & Aufbewahrung \\
\hline Hand- \& Saugfang & $\rightarrow$ & trocken bei $-24^{\circ} \mathrm{C}$ \\
\hline Kempson $\mathrm{H}_{2} \mathrm{O}$ & gesättigte Kochsalzlösung $\left(\mathrm{NaCl}_{\mathrm{aq}}\right)$ & trocken bei $-24^{\circ} \mathrm{C}$ \\
\hline $\begin{array}{l}\text { Kempson pKs \& } \\
\text { MacFadyen }\end{array}$ & $\begin{array}{l}\text { 40\%ige Trinitrophenol-Lösung (Pi- } \\
\text { krinsäure), }\end{array}$ & $70 \%$ (vol.) Ethanol \\
\hline O’Connor & Leitungswasser, z.T. kochend & $37 \%$ Formaldehyd \\
\hline Bodenfalle $\mathrm{H}_{2} \mathrm{O}$ & gesättigte Kochsalzlösung $\left(\mathrm{NaCl}_{\mathrm{aq}}\right)$ & trocken bei $-24^{\circ} \mathrm{C}$ \\
\hline Bodenfalle Gly & $\begin{array}{l}\text { 50\% \%ige Dihydroxydiethlether-Lösung } \\
\text { (Diethylenglykol) }\end{array}$ & $70 \%$ (vol.) Ethanol \\
\hline
\end{tabular}


Die Ameisen habe ich zusätzlich durch direkte Nestsuche und Köderschalen mit Honigwasser erfasst; die Nesterfassung wäre bei der reich strukturierten Streuschicht allerdings nur bei komplettem Umgraben quantitativ gewesen, worauf ich mit Rücksicht auf die geringe Flächengröße verzichtet habe. Mit den Köderschalen gelang für stark oberflächenaktive Ameisen-Arten eine brauchbare AktivitätsAbschätzung; für Arten mit versteckter Lebensweise sind allerdings nur die Kempson-Proben quantitativ zuverlässig, da Bodenfallen-Fänge nur qualitative und auch Saugproben allenfalls halb-quantitative Daten liefern.

\subsection{Bestimmung der Tiere und ihrer Biomasse}

Die Tiere wurden unter einem Stereobinokular aussortiert und, z.T. mit einem Mikroskop, auf Familien- bzw. Artniveau determiniert. Grundlage für die Bestimmung der Ordnungen und Familien war Schaefer (2000).

Für Insektenlarven wurden Chu (1949) und Peterson (1962), für Lumbricidae Graff (1953) und Sims \& Gerard (1985), für Isopoda Gruner (1966) und für Opilionida Martens (1978) hinzugezogen.

Die Ameisen wurden mit Seifert (1996), ergänzt durch Kutter (1977 \& 1978), Seifert (1983, 1988 \& 1991) und Czechowski et al. (2002), bestimmt.

Die Bestimmung adulter Spinnen erfolgte zu einem großen Teil zusammen mit Dirk Sanders und Alexander Sührig nach Heimer \& Nentwig (1991) und Roberts (1993 \& 1995), ergänzt mit Kronestedt (1990). Die Zikaden wurden von Ingke Rachor und Dr. Herbert Nickel determiniert.

Die Biomasse von Ameisen, Spinnen und Zikaden wurde artspezifisch jeweils an mehreren Individuen nach Trocknung $\left(60^{\circ} \mathrm{C}, 72 \mathrm{~h}\right)$ mit einer Feinwaage auf 10 $\mu \mathrm{g}$ genau gewogen.

\subsection{Chemische und bodenbiologische Analysen}

Zur Analyse von Bodenlösungen wurden Standardverfahren angewandt (Destillation des mineralischen Stickstoffs nach Kjeldahl, Kolorimetrie eines PosphatFarbkomplexes, pH-Werte in 0,01 mol/1 CaCl 2 -Lösung). Die Gehalte an Stickstoff und Kohlenstoff in Festproben (Bodenproben, Pflanzenteile) wurden nach Trocknung $\left(105^{\circ} \mathrm{C}, 2 \times 24\right.$ h), Homogenisierung in einer Schwingmühle und Einwaage von etwa 1000-2000 $\mu \mathrm{g}$ gaschromatographisch mit einem Carlo-ErbaElement-Analysator gemessen.

Bodendichte und Wassergehalt habe ich gravimetrisch $(24 \mathrm{~h}$ Trocknung bei $105^{\circ} \mathrm{C}$ ) bestimmt. Die Höhe der Streuschicht wurde direkt an den Bodensäulen gemessen.

Der Kalkgehalt $\left(\mathrm{CaCO}_{3}\right)$ wurde aus dem nach Salzsäurebehandlung entstandenen $\mathrm{CO}_{2}$-Volumen berechnet $(3 \mathrm{~g}$ TG Boden $+10 \mathrm{ml} \mathrm{25 \%} \mathrm{HCl}$ mit $7 \% \mathrm{Fe}$ - 
$\mathrm{SO}_{4} \bullet 7 \mathrm{H}_{2} \mathrm{O} ; \mathrm{Fe}^{2+}$ reduziert evtl. vorhandenes Mangandioxid, das sonst das Chlorid zu Chlor oxidieren könnte, welches auch die organische Substanz angreifen würde, Loeppert \& Suarez 1996). Die mittlere Standardabweichung der Eichkurven lag bei etwa 2,5\%. Zum Vergleich wurden aus mit Salzsäure ohne $\mathrm{Fe}^{2+}$-Zugabe behandelten Bodenproben die Gesamtkohlenstoff- und Stickstoff-Konzentrationen analysiert.

Die Messung der Basalatmung und Bestimmung der mikrobiellen Biomasse nach der Methode der Substrat-induzierten Respiration (SIR-Methode nach Anderson \& Domsch 1978) erfolgte in einer von Scheu (1992) modifizierten automatischen elektrolytischen $\mathrm{O}_{2}$-Mikrokompensationsanlage. Der Vorteil dieser Anlage besteht darin, dass die mikrobielle Biomasse und der Verlauf der Atmungskurven hier aus nur 1-2 g Boden bestimmt werden können. Die mikrobielle Biomasse wurde aus der Atmungsaktivität vor der Wachstumsphase (MIRR) nach dem von Beck et al. (1997) vorgeschlagenen Wert von $38 \mu \mathrm{g} \mathrm{C}_{\text {mic }}$ pro $\mu$ l verbrauchtem $\mathrm{O}_{2}$ pro Stunde berechnet. Die Ergosterolgehalte wurden nach alkoholischer Extraktion mit der HPLC gemessen (Djajakirana et al. 1996).

\subsection{Pflanzenwachstum und Mikrokosmen}

Als Laborsysteme wurden dynamische Mikrokosmen verwendet, die eine Drainage unter naturnahen Bedingungen erlauben (Wolters 1989, Scheu 1990). Zur gleichzeitigen Haltung von Ameisen wurden geeignete Versuchsgefäße neu entwickelt (Kap. 6). Im Freiland wurden Kunststoffröhren von $100 \mathrm{~mm} \varnothing$ und $200 \mathrm{~mm}$ Höhe mit vier 50x100mm großen Seitenöffnungen, die mit einer Gaze von ca. 5 $\mathrm{mm}$ Maschenweite verschlossen waren und den Ameisen und meisten anderen Bodentieren weitgehend ungehinderten Zutritt erlaubten, mit der Oberkante bündig zur Oberfläche eingegraben. Die jungen Pflänzchen wurden mit einem Drahtgitter vor Verbiss geschützt.

Die Pflanzenwurzeln aus den Mikrokosmen und aus Stechzylinderproben wurden über einer Siebkaskade ( $4 \mathrm{~mm}, 2 \mathrm{~mm}, 1 \mathrm{~mm}, 0,25 \mathrm{~mm}$ ) gewaschen und durch Handlese aussortiert.

\section{5. ${ }^{15} \mathrm{~N}$ und ${ }^{13} \mathrm{C}$-Analysen}

Die Pflanzen- und Tierproben wurden direkt gesammelt und bis zur Einwaage tiefgefroren. Die Isotopenverhältnisse in den Proben wurden extern im Kompetenzzentrum Stabile Isotope (KOSI) des Forschungszentrums Waldökosysteme der Universität Göttingen mit einem computergesteuerten gekoppelten System aus einem Carlo-Erba-Element-Analysator (NA 2500) und einem Gas-IsotopenMassenspektrometer (Finnigan Deltaplus) bestimmt, mit dem sowohl die Anteile der Kohlenstoff- wie auch der Stickstoff-Isotopen in einer Probe gemessen werden können (Reineking et al. 1993). Das Verhältnis des jeweils schwereren zum 
leichteren Isotop wird üblicherweise als Abweichung in Promille vom Isotopenverhältnis der jeweiligen Referenzsubstanz angegeben, für Kohlenstoff das $\mathrm{CaCO}_{3}$ des „Peedee Belemnite“ Gesteins, für Stickstoff der Luft-Stickstoff. Die mittlere Standardabweichung der internen Standards (Acetanilid) lag für Kohlenstoff und Stickstoff unter $0,1 \%$.

\subsection{Statistische Analysen}

Von allen Daten wurden die Residuen berechnet, mit dem Shapiro-Wilk-Test auf Normalverteilung getestet und gegen die Erwartungswerte (=GruppenMittelwerte) aufgetragen, um die Verteilung und Homogenität der Varianzen graphisch abschätzen zu können. Die bei Individuenzählungen von Tieren oft zu beobachtende Zunahme der Varianz mit steigenden Mittelwerten zeigt multiplikative oder exponentielle Zusammenhänge an. Besonders bei den Ameisendaten konnte ich diesen Effekt oft beobachten (Abb. 9). Durch die soziale Lebensweise ist die Erklärung besonders offensichtlich, eine Ameise kommt sozusagen selten allein. Durch logarithmische $(\log 10(x+1))$ oder Wurzeltransformation $\left((x+3 / 8)^{1 / 2}\right)$ konnte in fast allen Fällen Homogenität der Varianzen erreicht werden, die mit dem Fmax-Test (Köhler et al. 2002) oder dem Levene-Test statistisch abgesichert wurde.

Bei der optischen Analyse der Residuen fielen auch gleich Ausreißer auf, die auf Plausibilität und potentielle Fehler bei der Dateneingabe geprüft wurden.
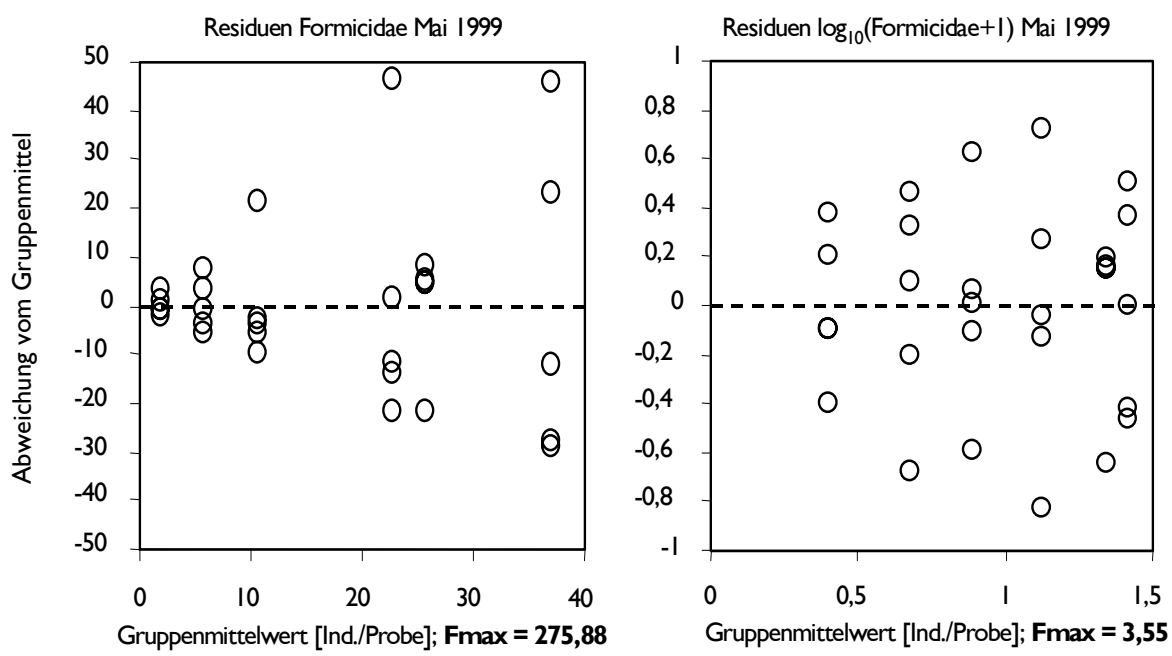

Abb. 9: Residuen der Ameisen-Individuenzablen des Klimaversuchs aus den Kempson-Proben vom Mai 1999 aufgetragen gegen die Erwartungswerte; links absolute, rechts log-transformierte Daten. 
Zur Überprüfung der experimentellen Effekte wurden Varianzanalysen durchgeführt, für Mittelwertvergleiche Tukey's HSD benutzt; die jeweiligen Modelle werden zusammen mit den Versuchsanlagen in den jeweiligen Teilkapiteln genauer angegeben.

Direkte Gradientenanalysen von zusammen erhobenen multivariaten Tier- und Umweltparameter-Datensätzen wurden mit Redundanz- (RDA) oder Kanonischen Korrespondenzanalysen (CCA) durchgeführt. Zuvor wurde mit einer „detrended“ (by segments) Korrespondenzanalyse (DCA, Hill \& Gauch 1980) die Gradientenlänge bestimmt; wenn der größte erfasste Gradient zwischen den Probenahmepunkten kleiner als zwei Standardabweichungen der reskalierten Gaußschen Glockenkurven der Arten war, also weniger als die Hälfte eines kompletten unimodalen Anstiegs und Abfalls umfasste, wurden die linearen Verfahren angewandt: Hauptkomponenten- (PCA) und Redundanzanalyse (ter Braak 1995).

Die statistischen Analysen wurden mit den Computerprogrammen SAS 8.1 (SAS Institute 2000), Statistica 6.0 (StatSoft 2001), Canoco 4.5 (ter Braak \& Smilauer 2002) und Excel 2000 (Microsoft 1999) durchgeführt. 


\section{Themenbereich I \\ Umwelteinflüsse und Tiergemeinschaften}

\section{Verteilungsmuster und Diversität von Ameisen, Spinnen und anderen Bodentieren in einem Gra- dienten vom Mischwald zum Trockenrasen auf Kalkgestein}

\subsection{Einleitung}

Die meisten Ameisenarten sind mehr oder weniger xerothermophil (Seifert 1996); entsprechend leben sie in Mitteleuropa in somnenexponierten Biotopen wie z.B. Trockenrasen oder lichten Wäldern in süd-exponierten Hanglagen. Buchenforste mit durchgehend geschlossener Kronendecke gehören dagegen zu den Ameisenärmsten Lebensräumen. Seifert (1996) charakterisiert beispielsweise 79 der 111 bisher in Deutschland festgestellten Ameisenarten wenigstens teilweise als Offenlandarten; die maximalen Biomassen der dominanten Arten können auf einem Hektar Wiese mit 110 bis $150 \mathrm{~kg}$ mehr als das Dreifache der Werte aus Optimallebensräumen der auffälligeren und sehr volkreichen Waldameisen aus der Formica rufa-Gruppe (ca. $40 \mathrm{~kg} / \mathrm{ha}$ ) erreichen. Ökologische Grundlagen sind bisher bei Waldameisen am umfassendsten erforscht (Übersichten z.B. bei Otto 1967, Gösswald 1990, Whittaker 1991, Way \& Khoo 1992); die Rolle von Ameisen in europäischen Grünland-Ökosystemen wurde vorwiegend in Polen und England intensiver bearbeitet (Übersichten z.B. bei Pêtal 1978, Woodell \& King 1991, Elmes 1991). 
Für Ameisen spielt in optimalen Lebensräumen die Konkurrenz um Raum in Form geeigneter Nistplätze (Strukturen, Mikroklima, Bodeneigenschaften) und als Territorium zur Nutzung der oft geklumpt verteilten Ressourcen eine entscheidende Rolle (Hölldobler \& Wilson 1990). Inter- und intraspezifische Konkurrenz bewirken oft eine Populationsstabilisierung der entsprechenden Arten (Pêtal 1981, Pontin 1961). Für Myrmica- und Lasius- Artenpaare beschrieben Brian (1983) bzw. Pontin (1961 \& 1963) die Ausbildung einer Dominanzhierarchie und das Ausweichen der unterlegenen Arten auf die Nutzung suboptimaler Ressourcen; Rosengren (1986) konnte in einem Freilandexperiment klar indirekte positive Effekte der dominanten Art Formica truncorum auf die subdominanten Arten Lasius flavus und Tetramorium spec. durch Verdrängung der intermediären Art Lasius niger, die ohne Anwesenheit von Formica $t$. mit den subdominanten Arten stark um die gleichen Ressourcen konkurrierte, feststellen.

Hügelbauende Arten haben in der Wahl ihres Nistplatzes größere Freiheit als Arten, die auf speziellere Nistplätze angewiesen sind, und reduzieren bei größerer Dichte die intraspezifische Konkurrenz neben Reduktion der Koloniegrößen vor allem durch eine regelmäßigere hexagonale Verteilung ihrer Nester (Waloff \& Blackith 1962, Elmes 1974).

Die räumliche Verteilung von Organismen, gerade von Schlüsselorganismen, ist wesentlich für das Verständnis von Gemeinschafts-Interaktionen, Mustern der Populationsdynamik und Ökosystemprozessen (Christ \& Wiens 1996). Ein großer Teil der Aktivitäten von Ameisen, besonders mögliche Bottom-up-Wirkungen, konzentrieren sich im Bereich der Nester, die meist über Jahre hinaus am selben Ort bleiben. Aufgrund dieser Immobilität eignen sich Ameisennester auch besonders für eine räumliche Auswertung (Walloff \& Blackith 1962, Christ \& Wiens 1996) sowie als Indikatoren für länger wirkende Umweltveränderungen (King 1981, Seifert 1998).

Die Verteilung der Ameisenarten auf verschiedene Biotope oder auf Mikrohabitate entlang von Gradienten wechselnder Umweltfaktoren wurde zwar schon häufiger untersucht (in Deutschland z.B. von Seifert 1982, Assing 1986, Bauschmann 1988), dennoch ist der faunistische Erfassungsstand für viele Regionen, darunter insbesondere Südniedersachsen und Nordhessen, noch sehr niedrig (Assing 1994, Rögener \& Pfau 1994, Bauschmann et al. 1996).

Um die räumliche Verteilung der Ameisen zusammen mit anderen Tieren, insbesondere der Bodenmakrofauna, und ihre Abhängigkeiten von verschiedenen Umweltfaktoren zu erfassen, habe ich im Untersuchungsgebiet eine Fläche mit vielen unterschiedlichen Habitatausprägungen und Sukzessionsstufen ausgewählt und mit einem räumlichen Raster mehrere dieser Gradienten abgedeckt. Folgende Fragen standen dabei im Zentrum:

1. Welche Ameisenarten leben dort unter welchen ökologischen Bedingungen und wie verteilen sie sich im Raum?

2. Welche Korrelationen bestehen zur übrigen Tiergemeinschaft, welche mikroklimatischen, pedologischen und biologischen Umweltfaktoren spielen dabei eine Rolle? 
3. Gibt es tatsächlich positive Interaktionen mit der Bodenfauna und negative mit anderen Prädatoren? Können dabei soziale Insekten Tiere mit solitärer Lebensform an den „ökologischen Rand“ drängen?

4. Welche Rolle spielen Sukzessionsprozesse und können daraus naturschutzfachliche Empfehlungen abgeleitet werden?

Die räumliche und multivariate Analyse erlaubt es, die sehr komplexen populationsökologischen Daten auf verschiedenen Skalenebenen zusammen mit relevanten Umweltparametern raumbezogen auszuwerten und damit ökologische Modelle im Freiland adäquater zu überprüfen.

\subsection{Methodik}

Raster

Auf einem brachliegenden Halbtrockenrasen an einem Mischwaldrand auf einem Südwest-exponierten Kalkhang im Werratal („Wiese 1“, Kap. 3) wurde im Frühjahr 1998 ein hexagonales 5 m-Raster angelegt. Beginnend an einer Kiefer am Waldrand wurde das Raster $10 \mathrm{~m}$ weit hangaufwärts, $10 \mathrm{~m}$ nördlich und $20 \mathrm{~m}$ südöstlich in den Wald hinein, $25 \mathrm{~m}$ weit südlich bis zum ehemaligen Waldrand etwa auf der Grenze des Grundstücks und $45 \mathrm{~m}$ weit den Hang hinunter komplett über den Halbtrockenrasen und eine trockene Glatthaferwiese hinweg bis in die umliegenden Gebüsche hinein eingemessen. Entlang von 8 Reihen, die in $240^{\circ}$ WestSüdwest-Richtung geradlinig den Hang hinunter liefen, wurden insgesamt 82 Untersuchungspunkte markiert (Abb. 10). Alle Rasterpunkte (bis auf den ersten an der willkürlich ausgewählten Kiefer) lagen - durch die jeweils genau $5 \mathrm{~m}$ Abstand zu den sechs benachbarten - rein zufällig in unterschiedlichen Mikroausprägungen der verschiedenen Habitate.

Zusätzlich wurde von der nördlichen Ecke des Halbtrockenrasens aus auf der Nachbarwiese („Wiese 1a“, Kap. 2.4.) ein linearer Gradient am Waldrand entlang angelegt, zunächst $15 \mathrm{~m}$ nach Norden, anschließend $20 \mathrm{~m}$ weit in $300^{\circ}$ WestNordwest-Richtung, bis zu einer Kolonie von Formica pratensis Retzius reichend. Jeweils im Abstand von 5 Metern und zusätzlich kurz vor und hinter einem der Nesthügel wurden zusammen 9 Probenahmepunkte markiert (Abb. 10).

19 Rasterpunkte lagen im Buchen-Kiefern-Mischwald, z.T. mit Hasel und Lärche (Abkürzung in den Ordinationen: Wald), 8 am südwestlichen oder westlichen Waldrand mit Abendsonne, 18 auf den noch offenen Bereichen des Halbtrockenrasens, 6 auf der Glatthaferwiese, 7 im Schlehdorngebüsch (Schlehe), 5 im Kiefernjungwuchs (Kiefernjw), 10 in Hartriegelbeständen, 6 im unteren Hain aus Feldahorn, Weißdorn und Kirsche (Hain), 5 unter einzeln stehenden Heckenrosen (Rosa) und weitere 5 im Mittagsschatten einzeln stehender Büsche oder junger Bäume (Busch). 


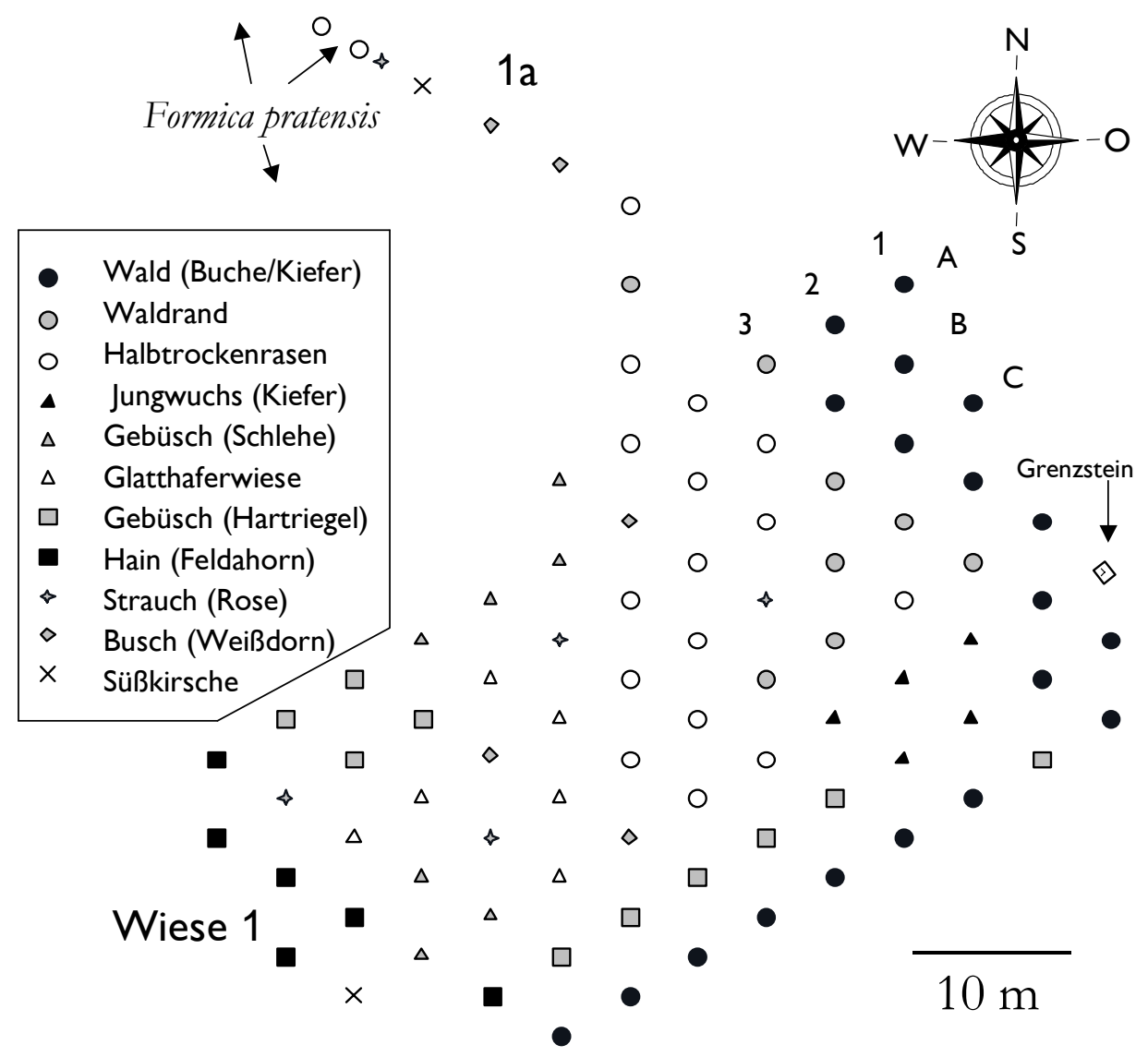

Abb. 10: Verteilung der Rasterpunkte auf Wiese 1; Legende der Symbole für die Habitattypen.

Probenabme und Erfassung ergänzender Daten

Am 3. und 4. Mai 1998 wurde an allen 91 eingemessenen Punkten jeweils eine Kempsonprobe genommen, der Lebensraumtyp bestimmt und Vegetationsstruktur und Beschattungsgrad abgeschätzt. Nachdem die Tiere ausgetrieben waren, wurde von jeder 72 Stunden bei $60{ }^{\circ} \mathrm{C}$ getrockneten Bodensäule die Pflanzenbiomasse und Streuauflage sortiert und gewogen. Die Erde wurde gesiebt und wie die ausgesiebten Steine gewogen. Von je einer Teilprobe wurden der pH-Wert (in $\mathrm{CaCl})$ und der Kalkgehalt $\left(\% \mathrm{CaCO}_{3}\right)$ gemessen.

Im folgenden Sommer habe ich mit Honigwasser-Ködern die Ameisenaktivität an verschiedenen Stellen der Fläche abgeschätzt und die Nester der angelockten Arbeiterinnen gesucht.

Im Sommer 1999 wurden 20 große Schraubgläser (5,5 cm ø mit Ringaufsatz) und 61 kleine Rollrandschnappdeckelgläser $(2,2 \mathrm{~cm} \mathrm{ø,} 50 \mathrm{ml})$ als Bodenfallen auf der Fläche eingegraben; die kleinen Fallen standen dabei direkt an ausgewählten 
Punkten des Rasters, die großen an repräsentativen Stellen der sieben wichtigsten Lebensraumtypen: Je 4 im Wald, auf dem Trockenrasen und auf der Brachwiese, je 2 am Waldrand, im Kiefernjungwuchs, Schlehengebüsch und Feldahorn-Hain. Der Wassergehalt der ausgestochenen Bodenproben wurde durch Trocknen und Wiegen bestimmt.

Von 1999 bis 2000 wurden auf der Fläche Nahrungsnetzanalysen (Kap. 6), bodenbiologische Untersuchungen und Experimente (Kap. 8 \& 9) und 2002 ein Ausschlussversuch (Kap. 7) durchgeführt, so dass weitere Arten und Daten gesammelt werden konnten, die allerdings nicht mit in die statistische Analyse eingehen.

\section{Mikroklima-Messungen und Analyse der Baumkronenstruktur}

Durch die mittlerweile zur Verfügung stehende bessere technische Ausrüstung konnten nochmals im Frühjahr 2002 Temperatur- und Luftfeuchte-Messungen mit elektronischen Dataloggern der Firma Hobo und im Sommer 2003 Digitalfotos der Rasterflächen und der sie überdeckenden Baumkronen bzw. Gebüsche gemacht werden. Die Datalogger standen an zwei Messperioden vom 3. bis 18. April an den 10 m-Rasterpunkten und vom 18. bis 22. April an weiteren Punkten des Rasters. Aus den Daten wurden Mittelwerte, Maxima und Minima berechnet.

Chemische Reaktionsgeschwindigkeiten steigen aber nicht linear mit der Temperatur, sondern hängen nach der Arrheniusgleichung exponentiell von dem Verhältnis der Aktivierungsenergie zur mittleren thermischen Translationsenergie ab. Wechselwarme Organismen sind in ihrer Lebensentfaltung ebenso direkt von der Temperatur abhängig. In Unkenntnis der relevanten Enzymreaktionen und ihrer Aktivierungsenergien ist die Van't Hoffsche Reaktions-GeschwindigkeitsTemperatur-Regel die beste Näherung, wonach eine Erwärmung um $10{ }^{\circ} \mathrm{C}$ eine Verdoppelung bis Verdreifachung der Geschwindigkeit bedeutet. Die Temperatur $\mathrm{T}$ in ${ }^{\circ} \mathrm{C}$ habe ich entsprechend einer vorsichtigen Näherung $2^{\mathrm{T} / 10}$-transformiert, gemittelt und rücktransformiert, um einen Mittelwert „RGT“ zu erhalten, der diese Zusammenhänge besser berücksichtigt.

Die Daten beider Messperioden wurden mit den Daten zweier Referenzpunkte rechnerisch aneinander angeglichen (lineare Umrechnung der in Kelvin transformierten Werte mit dem Verhältnisfaktor der Referenz-Periodenwerte zu den Werten des gesamten Messzeitraums).

Zur objektiven Messung des Beschattungsgrades bzw. der Baumkronendichte wurde die Farbtiefe von senkrecht nach oben aufgenommenen digitalen Fotos (Nikon Coolpix 4500 (1:2,6; 7,85 mm); HP Photo Smart 618 (1:2,4; 5,2 mm), Weitwinkel, $2272 \times 1704$ Pixel im JPEG-Dateiformat) mit dem Computerprogramm Paint Shop Pro 4.15 (Jasc Software 1999) auf 2 verringert und wieder auf 16 Mio. erhöht, um den Maximalwert der Lumineszenz ablesen zu können, der dem Weiß- bzw. Schwarzanteil der Bilder proportional ist. Bei nicht zu starker Bewölkung wurden alle aufgenommenen Vegetationsstrukturen sehr detailgetreu als schwarze Pixel von den weißen des durchscheinenden Himmels getrennt. Die Ergebnisse dieser Messung korrelierten sehr gut mit den fünf Jahre zuvor abge- 
schätzten Werten der Beschattung, was den Eindruck einer nur sehr langsam voranschreitenden Verbuschung bestätigte.

Für Ameisen wird die maximale Bodentemperatur als besonders relevante Kenngröße angesehen (Seifert 1986). In Anlehnung an Seifert habe ich die an unterschiedlichen, aber immer sonnigen Tagen nachmittags mit batteriebetriebenen Digitalthermometern $5 \mathrm{~cm}$ unter der Bodenoberfläche gemessenen Temperaturen auf Standardbedingungen umgerechnet. Tageszeitliche Abweichungen wurden durch regelmäßiges Ablesen von Thermometern an Referenzpunkten habitatspezifisch ausgeglichen.

Die Messungen an unterschiedlichen Tagen wurden mit den Daten der Klimastation des DWD in Kassel auf einen einheitlichen Maximalwert zurückgerechnet. Aus den Regressionsgraden von Messserien im Boden eingegrabener Datalogger (Kap. 5) mit verschiedenen Klima-Kenngrößen am gleichen Tag in Kassel konnten in Abhängigkeit von der Beschattung und Vegetationsdichte drei Rechenformeln erstellt werden, in denen das unterschiedliche Erwärmungs- und Abkühlungsverhalten verschiedener Habitattypen berücksichtigt wird (Tab. 3). Jedem Rasterpunkt wurden relative Anteile der drei extremen thermischen Verhaltensausprägungen zugeordnet, die aus dem Beschattungsgrad in der südlichen Hälfte des Himmel-Fotos und bei weniger als $35 \%$ Beschattung auch aus der Krautschichtbiomasse berechnet wurden.

Tabelle 3: Relative Anteile (je 1/12) der Klima-Kenngrößsen 3-Tages-Mittel ( $\left.T_{3 d M i t}\right)$, Tagesmittel (T $\left.T_{\text {Mit }}\right)$, Temperatur um 12 und $18 \mathrm{Ubr}$ (T12 brw. T18), Maximaltemperatur (TMax), Sonnenscheindauer (Son) und relativer Luftfeuchte $(r F)$ an den Regressionsformeln zur Berechnung der Standard-Maximal-Bodentemperatur

\begin{tabular}{l|ccccccc}
\hline & $\mathrm{T}_{3 \mathrm{dMit}}$ & $\mathrm{T}_{\mathrm{Mit}}$ & $\mathrm{T}_{12}$ & $\mathrm{~T}_{18}$ & $\mathrm{~T}_{\mathrm{Max}}$ & Son & $\mathrm{rF}$ \\
\hline Maximale Beschattung & 3 & 3 & 2 & & 1 & 2 & -1 \\
Wiese & 1 & & & 3 & 3 & 3 & -2 \\
Annähernd blanker Boden & & & 2 & 1 & 3 & 4 & -2 \\
\hline
\end{tabular}

\section{Statistische Auswertungen}

Mit den Deckungsgraden der Pflanzenarten im mittleren Abschnitt, den Individuenzahlen aller erfasster Tiergruppen und denen der Ameisenarten aus den Bodenproben - zusammen mit den aufgenommenen Umweltvariablen - wurden multivariate direkte Gradientenanalysen (Kap. 3.6) durchgeführt. Folgende Umweltvariablen gingen als erklärende Faktoren in die Analyse ein [Einheit] (Abkürzung in den Ordinationen):

- der Anteil der Steine in den oberen $5 \mathrm{~cm}$ des Bodens [gew \%] (Steine)

- Bodendichte $\left[\mathrm{kg} / \mathrm{l}=\mathrm{g} / \mathrm{cm}^{3}\right]$ (Dichte)

- Protonenkonzentration $\left[-\log _{10}\left[\mathrm{H}^{+}\right]\right](\mathrm{pH})$

- Kalkgehalt der oberen $5 \mathrm{~cm}$ des Bodens [gew \% $\mathrm{CaCO}_{3}$ ] (Kalk) 
- Bodentemperatur in $5 \mathrm{~cm}$ Tiefe, auf vergleichbare Maximalwerte interpoliert $\left[{ }^{\circ} \mathrm{C}\right]$ (Tbod)

- Thermisches Verhalten [relative Skala von -1 für langsamste Änderungen bei maximaler Beschattung und Windschutz über 0 für dichtbewachsene, rel. unbeschattete Glatthaferwiese bis +1 für offene Hangbereiche mit minimaler Vegetationsdecke und dementsprechend schnelleren und stärkeren Temperaturschwankungen] (Therm)

- Beschattungsgrad [\% Himmelsabdeckung in Südrichtung] (Schatten)

- Trockengewichte von Moos, Gras, Kraut, Buchenblättern, anderen Laubblättern (Hasel, Weißdorn usw.) und Kiefernnadeln (Moos, Gras, Kraut, Buche, Laub, Kiefer)

- Trockengewichte von holzigen Stängeln der Krautschicht, Holz, KiefernRinde und Kiefern- bzw. Lärchenzapfen $\left[\mathrm{g} / 346 \mathrm{~cm}^{2}\right]$ (Stängel, Holz, Rinde, Zapfen)

- Anzahl Sonderstrukturen [Anzahl/346 $\mathrm{cm}^{2}$ ] Haselnüsse, Buchensamen, Eicheln, Kirsch- und Hartriegelkerne (Nuss), Bucheckernhülsen (BuEcker) und Schneckenhäuser (Schnecke)

Jede Probenahmestelle wurde einem Habitattyp zugeordnet (s.o.). Von diesen nominalen Umweltfaktoren wurden die Zentroide entsprechend ihrer Korrelation mit den zuvor berechneten Arten- oder Arten-Umwelt-Achsen aufgetragen, ohne dass sie die Achsenbildung mit beeinflusst hätten (passive Variablen oder supplementary data). Zur gleichzeitigen Abschätzung der Heterogenität dieser Habitattypen sind die Ordinationspunkte der einzelnen Standorte (sample scores) in den Abbildungen entsprechend durch Symbole gekennzeichnet.

Durch automatische Vorwärts-Selektion der Umweltvariablen kann festgestellt werden, wie stark der Einfluss der einzelnen UV allein ist, in welcher Reihenfolge sie die jeweils maximale zusätzliche Varianz erklären und welche der Variablen signifikante Beiträge zum Modell liefern (Monte-Carlo-Test).

Eine weitere direkte Gradientenanalyse nur mit der räumlichen Lage der Probenahmepunkte, also ihren beiden x- und y-Koordinaten als erklärende Umweltvariablen, gibt zum einen an, welchen Anteil allein die räumliche Lage an der Varianz der Tiergemeinschaft hat, und ermöglicht zum anderen bei einer Auftragung der Ordinationswerte der Probenahmepunkte, die eine lineare Kombination der Umweltvariablen darstellen, eine Abschätzung, in welchen Bereichen des Rasters die Schwerpunkte der Habitattypen und der jeweiligen Tiergruppen liegen.

Zur statistischen Absicherung wurde jeweils ein Monte-Carlo-Test mit 999 nicht restriktiven Permutationen durchgeführt. Allerdings wäre ein rein zufälliges Permutieren der Proben bei hoher räumlicher Autokorrelation nicht angemessen. Die Güte der kanonischen Ordination der Siedlungsdichten der Tiere zusammen mit den Umweltfaktoren wurde, unabhängig von einer eventuell signifikanten Autokovarianz-Funktion, durch einen Monte-Carlo-Test mit restriktiven Permutationen für eine rasterförmige Versuchsanlage für das Kernraster überprüft. 


\subsection{Ergebnisse}

\subsubsection{Biodiversität}

Schon beim ersten Betreten der Flächen fällt die große Vielfalt auf: Karge, sonnenexponierte Bereiche wechseln ab mit dichtbewachsenen oder verbuschten Bereichen, viele Pflanzenarten prägen eine schon optisch vielfältige Struktur. Auch der säumende Gehölzgürtel enthält auffallend viele Arten. Selbst auf wenigen Quadratmetern im Übergangsbereich vom Halbtrockenrasen zur Glatthaferwiese konnten schon an einem Kartierungstermin etwa 30 Gefäßpflanzenarten nachgewiesen werden (Tab. 1, Kap. 2). Direkt auf der Fläche können auch ohne Berücksichtigung der Unterarten der extrem arten-/ hybridenreichen Gruppen mindestens 12 Baum- und Straucharten unterschieden werden.

Auch die Tierwelt erscheint auf Anhieb sehr formenreich und bunt - sogar im engeren Wortsinne, denn ich konnte auch viele farbenfrohe Schmetterlinge und Käfer beobachten, die andernorts deutlich seltener zu sehen sind. Häufiger zu sehen waren unter anderem Sandlaufkäfer (Cicindela campestris L.) und ihre Larven, Immenkäfer (Trichodes apiarius (L.)), Pinselkäfer (Trichius fasciatus (L.)), Kaisermantel (Argynnis paphia (L.)), Nagelfleck (Aglia tau (L.)), Widderchen (Zygaena spec.) und verschiedene Bläulinge (Lycaenidae). Ebenfalls unter Schutz stehen die Hornissen (Vespa crabro L.), die unter zwei der Beobachtungsbrettchen des Ausschlussversuchs nisteten, die Libellen, die offenbar aus den Feuchtbiotopen in der Nähe eingeflogen kamen (Enallagma cyathigerum (Charpentier), Anax imperator Leach u.a.), der Hirschkäfer (Lucanus cervus (L.)), den ich am benachbarten Badenstein gesichtet habe, und die Zauneidechsen (Lacerta agilis L.), Blindschleichen (Anguis fragilis L.) und Schlingnattern (Coronella austriaca Laurenti), die auf der Fläche leben. Viele dieser Arten sind typisch für wärmebegünstigte, waldreiche Habitatverbünde; dazu zählen auch die Wanzenfamilien Plataspidae mit Coptosoma scutellatum (Geoffroy) (Kugelwanze) und Phymatidae mit Phymata crassipes (F.).

\section{Zikaden}

Im mittleren Bereich der Wiese 1, der Übergangszone vom Halbtrockenrasen in die Glatthaferwiese, konnten mit 175 Saugfängen und 50 Bodenproben mindestens 24 Zikadenarten nachgewiesen werden (Rachor 2004). Auf den im Sommer 2002 zusammen etwa $7 \mathrm{~m}^{2}$ abgesaugter bzw. beprobter Fläche konnten nur 135 adulte Zikaden gefunden werden. Die meisten Jugendstadien lassen sich noch nicht sicher einer Art zuordnen, so dass mit dem Vorkommen von weiteren Arten gerechnet werden muss.

Die häufigste Art Adarrus multinotatus (Boheman) (Gemeine Zwenkenzirpe) ist typisch für Trockenrasen. Arboridia parvula (Boheman) (Beilblattzikade) und Asiraca clavicornis (F.) (Schaufelspornzikade) gelten als gefährdet, Anaceratagallia venosa (Geoffroy) (Kleedickkopfzikade) steht auf der Vorwarnliste (Nickel \& Remane 2002). Asiraca wurde nach hundert Jahren erstmals wieder in dieser Region am äußersten Rand ihres Verbreitungsareals nachgewiesen. 


\section{Spinnen}

Im untersuchten Gradienten wurden in den ausgewerteten 56 Bodenproben (je 1/28 $\mathrm{m}^{2}$ ), 40 Bodenfallen- (zus. 4 Wochen) und 211 Saugfängen (je $1 / 28 \mathrm{~m}^{2}$ ) zusammen 75 Arten aus 22 Familien gefunden (Sanders 2004). Der Schwerpunkt dieser Proben lag auch auf dem Kernbereich der Wiese 1. Bemerkenswert ist die hohe Dichte der als gefährdet angesehenen Tapezierspinne Atypus piceus Sulzer von bis zu $80 \mathrm{Ind} . / \mathrm{m}^{2}$. Auch die Baldachinspinnen Notioscopus sarcinatus O.P. Cambridge und Theonina cornix Simon und die Bodenspinne Habnia candida Simon werden in der Roten Liste für Deutschland als gefährdet (RL 3) und die Springspinne Heliophanus dampfi Schenkel als stark gefährdet (RL 2) eingestuft (Platen et al. 1998). Die mediterran verbreitete Wolfsspinne Arctosa personata L. Koch 1872 c.f. ist bisher noch nicht in Deutschland gefunden worden (Platen et al. 1995, Blick et al. 2002). Eine Auflistung aller nachgewiesener Spinnenarten findet sich in Anhang A.

38 der Spinnenarten jagen ohne Netz (vagant); in den offenen Habitatbereichen übertreffen sie mit der 1,5-fachen Individuenzahl und sogar 4- bis 6-fachen Biomasse deutlich die hypergäischen Netzspinnen. Die größten Biomassen aller Spinnen hatten die eher sessilen und direkt am bzw. im Boden lebenden Spinnenarten Atypus piceus und Aulonia albimana Walckenaer, die zwar Fangschläuche bzw. Netze bauen, sich in ihrer Lebensweise aber doch deutlich von den anderen Netzspinnen (v.a. Linyphiidae und Araneidae) unterscheiden. Von den rein vaganten Spinnen hatte auf der untersuchten Fläche die Wolfsspinnengattung Alopecosa die höchste Biomasse.

\section{Ameisen}

Insgesamt konnten 18 Ameisenarten mit Nestern direkt auf der Rasterfläche nachgewiesen werden (Tab. 4), in direkter Nähe weitere 5 Arten und 1 Weibchen von Myrmica ruginodis ohne Arbeiterin, was noch kein Nachweis einer erfolgreichen Koloniegründung ist. Kolonien der Roten und der Kahlrückigen Waldameise konnten nur etwas entfernter am benachbarten Badenstein, allerdings an vergleichbaren Waldrandhabitaten, gefunden werden.

Polyergus rufescens, die dulotisch lebende (sklavenhaltende) Amazonenameise, fand ich in einem flachen Hügel zusammen mit „versklavten“ Arbeiterinnen der Arten Formica fusca und Formica cunicularia im mittleren Bereich der Wiese 1 in unmittelbarer Nachbarschaft zu Nestern von Lasius flavus und Myrmica sabuleti. Polyergus gilt aufgrund ihrer Seltenheit, aber auch ihrer besonderen Lebensweise, die an Arten der Untergattung Serviformica gebunden ist, und aufgrund ihrer Habitatansprüche als stark gefährdet (Rote Liste 2, Seifert 1998). 
Tabelle 4: Liste der auf der Untersuchungsfläche und in ibrer unmittelbaren Umgebung nachgewiesenen Ameisenarten (zu Angaben des Fundorts siebe Abb. 2; obne Angabe: Wiese 1).

\begin{tabular}{lll}
\hline & \multicolumn{1}{c}{ Formicidae } & Fundort (o.A.: Wiese 1) \\
\hline Ponerinae: & Ponera coarctata (Latreille 1802) & \\
\hline Myrmicinae: & Myrmica sabuleti Meinert 1860 & \\
& Myrmica schencki Emery 1894 & Wiese 2 \\
& Myrmica rubra Linnaeus 1758 & Wiese 2, nur O \\
& Myrmica ruginodis Nylander 1846 \\
& Solenopsis fugax (Latreille 1798) \\
& Leptothorax interruptus (Schenck 1852) \\
& Leptothorax tuberointerruptus Forel 1915 \\
& Leptothorax nylanderi (Förster 1850) \\
& Leptothorax parvulus (Schenck 1852) \\
& Stenamma debile (Förster 1850) \\
& Tetramorium caespitum (Linnaeus 1758) \\
& Myrmecina graminicola (Latreille 1802)
\end{tabular}

Dolichoderinae : Tapinoma erraticum (Latreille 1798)

Formicinae : Camponotus ligniperda (Latreille 1802)

Wiese 2

Polyergus rufescens (Latreille 1798)

Lasius alienus (Förster 1850)

Lasius niger (Linnaeus 1758)

« Maisfeld », Wiese 2

Lasius flavus (Fabricius 1781)

Lasius fuliginosus (Latreille 1798)

Wald, etwas oberhalb

Formica fusca Linnaeus 1758

Formica cunicularia Latreille 1798

Formica cinerea Mayr 1853

Formica pratensis Retzius 1783

Fläche 1a / 1b / 1c

Formica rufa Linnaeus 1761

Badenstein

Formica polyctena Förster 1850

Badenstein 
Gefährdete Arten (RL 3) sind Formica cinerea, Leptothorax interruptus, Leptothorax tuberointerruptus, Myrmica schencki, Ponera coarctata und Solenopsis fugax. Auf der Vorwarnliste stehen Formica pratensis, Leptothorax parvulus, Myrmica sabuleti und Tapinoma erraticum. Allein auf der Wiese 1 mit ihren Nachbarflächen konnten $20 \%$ der in Deutschland heimischen Ameisenarten nachgewiesen werden, davon sind ein Drittel in ihrem Bestand in Deutschland gefährdet, mit den Arten auf der Vorwarnliste sogar die Hälfte.

\subsubsection{Verteilung der Tiergruppen in Abhängigkeit von der Umwelt}

\section{Wald-Wiese-Gradient}

Die Tiergruppen aus den Bodenproben zeigten schon auf Familienniveau deutliche Unterschiede in ihrer relativen Zusammensetzung zwischen den verschiedenen Ausprägungen der untersuchten Lebensräume. Allerdings ist der insgesamt erfasste Gradient der Variation relativ kurz, die erste Achse einer detrended Korrespondenzanalyse (DCA) deckt zwischen den beiden unterschiedlichsten Probenahmeorten die 1,57-fache Standardabweichung der Gaußkurve ab, so dass die Ordination mit einem linearen Verfahren besser geeignet erscheint (Tab. 5).

Der Eigenwert der ersten Achse einer Hauptkomponentenanalyse ist 0,275, d.h. 27,5\% der Variation der Abundanzdaten der Tiergruppen kann durch diese rein mathematisch errechnete Achse oder „Hauptkomponente“ erklärt werden. Eine Auftragung der ersten beiden Achsen erklärt knapp $40 \%$ der Variation (Abb. 11). Allein aufgrund der relativen Individuen-Abundanzen der Tiergruppen werden die waldartigen deutlich von den eher offenen Lebensräumen unterschieden. Ameisen und alle Gruppen der Schnabelkerfe, sowie Erdläufer, Spinnen, Weberknechte, Kurzflügelkäfer und Asseln haben die Schwerpunkte ihrer Verteilungen auf den sonnigeren Flächen, hingegen sind Pseudoskorpione, Schnurfüßer, andere Hautflügler, Zweiflügler-Larven und die saprophagen Käfer deutlich mit dem Wald assoziiert. Die in der Ordination zusammengefassten Coleoptera waren zum Großteil Rhizophagidae, Catopidae, Nitidulidae, Cryptophagidae und Ptiliidae.

Der nächst größere Anteil der Varianz (12,4\%) wird durch einen Gradienten von den wärmeren, am Oberhang vorm Waldrand liegenden, zu den eher kühleren und feuchteren Habitattypen am Unterhang erklärt. Hier sind es die Ameisen und übrigen Hautflügler, Käferlarven (v.a. Cantharidae und Staphyliniformia, die nicht sicher den Staphylinidae zugeordnet werden konnten), Wanzen und Regenwürmer auf der einen, der exponierteren, und die Asseln, Tausendfüßer (Glomeridae und Julidae), saprophagen Käfer, Röhrenschildläuse, Weberknechte und Kurzflügelkäfer auf der anderen, der überwiegend durch dichtere Gehölze geschützteren, Seite. 


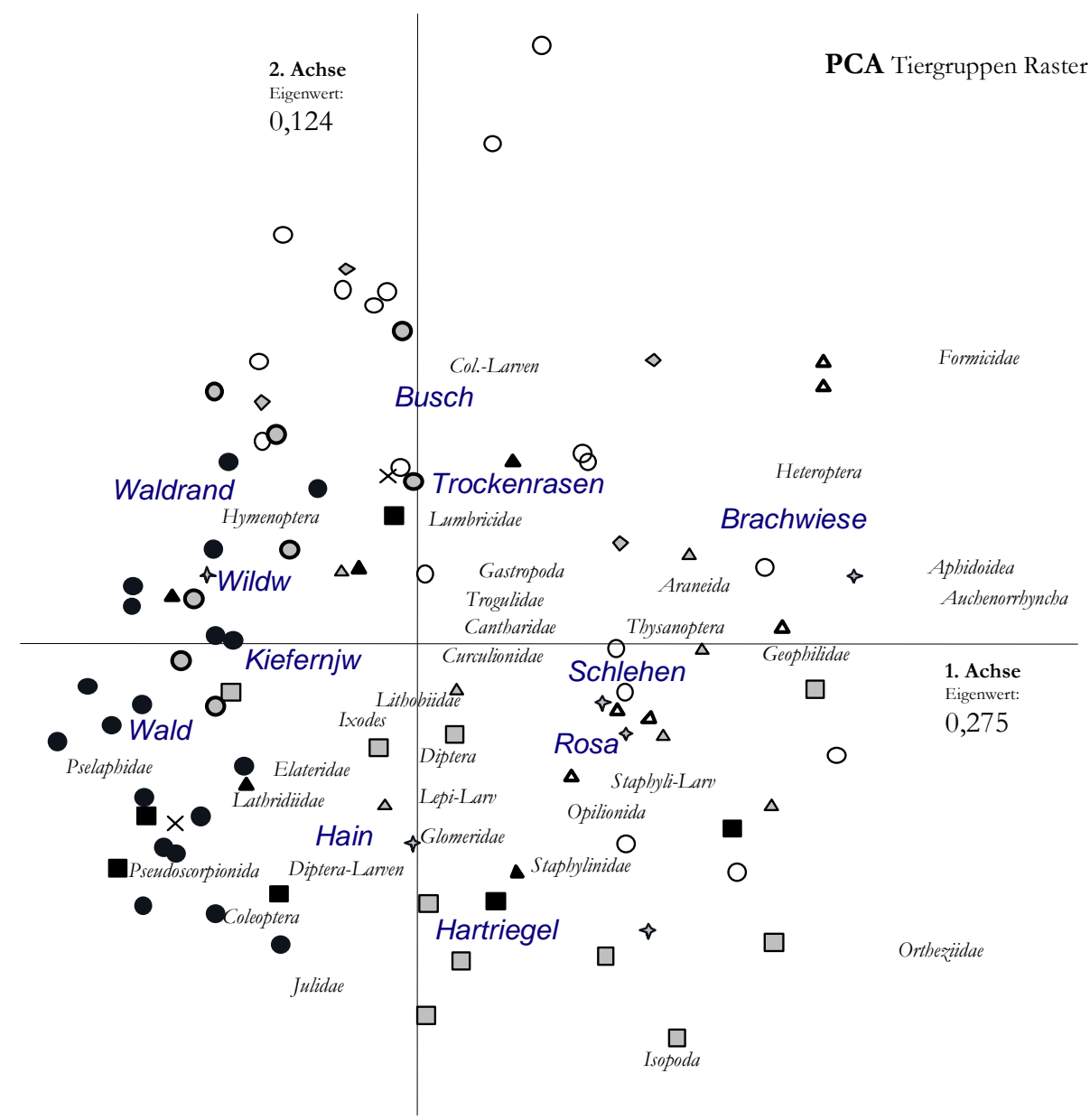

Abb. 11: Ordination der Siedlungsdichte verschiedener Tiertaxa und der Rasterpunkte entlang der beiden ersten Hauptkomponenten (Achsen der PCA). Die verschiedenen Habitattypen sind als passive Variablen aufgetragen; Legende der Symbole für die Rasterpunkte in Abb. 10, dunkle Symbole kennzeichnen beschattete Standorte; Coleoptera: weitere, überwiegend saprophage Käferfamilien; Elateridae: Schnellkeäfer-Larven; Staphyli-Larv: Kuraflügelkäfer-Larven; Col.-Larven: Larven anderer Käferfamilien; Lepi-Larv: Schmetterlingsraupen; Wildw: Wildwechsel; Kiefernjw: Jungwuchs Pinus sylvestris.

Werden die Umweltfaktoren als erklärende Variablen in die Ordination mit aufgenommen, sinkt der Eigenwert der ersten Achse auf 0,175 und derjenige der zweiten Achse auf 0,05 (Tab. 5), d.h. der Anteil der Tiergruppenvarianz, der durch die Auftragung der ersten beiden Achsen der RDA erklärt wird, sinkt auf 22,5\%. Das ist zwar nur knapp mehr als die Hälfte des Wertes der ersten beiden Hauptkomponenten, dennoch kennzeichnet gerade die erste Achse einen deutlichen Gradienten. Dieser Gradient trennt in erster Hinsicht die Wald- von den 
Wiesenhabitaten: Zur einen Seite hin (jetzt mit positiven Achsenwerten) liegen die Wald-Rasterpunkte mit den Pseudoskorpionen und Palpenkäfern und etwas intermediärer die übrigen saprophagen Käferfamilien, die Steinläufer und SchnurfüBer zusammen mit den Probenahmepunkten im Jungwuchs und am Waldrand. Positiv mit der ersten Achse korrelieren besonders die Umweltfaktoren Totholzmenge, Beschattung, die Menge an Buchenblättern, Kiefernnadeln und Rinde und die Zahl an Nüssen und Bucheckerschalen. Negativ mit dieser Achse korrelieren der $\mathrm{pH}$-Wert, die maximale Bodentemperatur, die Bodendichte, die thermischen Eigenschaften, der Kalkgehalt und die Menge an Moos, Gras und Stängeln. Mit den höchsten Werten dieser Umweltvariablen sind die Ameisen und alle Schnabelkerfe assoziiert. Zur Mitte hin folgen die Erdläufer, Fransenflügler und Spinnen mit dem Zentroid der Brachwiese. Intermediär werden der Trockenrasen und die verschiedenen Arten Gebüsche ordiniert.

Tabelle 5: Eigenwerte und Varianzaufklärungen (VA) der Artenvarianz. (DCA) bzw. der Arten-Umwelt-Relation (RDA) der ersten vier Achsen einer detrended Korrelationsanalyse (DCA), einer Hauptkomponentenanalyse (PCA) und von Redundanzanalysen (RDA) der Individuenzablen der Tiergruppen aus den Bodenproben der Rasteruntersuchung mit 20, 9 und 2 (den X,YKoordinaten) Umweltvariablen (UV). Die VA der Artenvarianz ist bei linearen Verfahren (PCA, $\mathrm{RDA}$ ) gleich dem Eigenwert der jeweiligen Achse. Bei DCA zusätzlich Angabe der Gradientenlänge (in SD), bei RDA der F-Werte und Signifikanzniveaus eines Monte-Carlo-Tests mit 999 nichtrestriktiven Permutationen (***: $p=0,001)$. $\mathrm{RD} A_{20}: \mathrm{RDA}$ mit allen $20 \mathrm{UV} ; \mathrm{RDA}$ : nur mit den 9 UV mit böherer Signifikanz. (Forward selection, Tab. 6); $R D A_{X Y}$ : nur mit den $X-Y$ Koordinaten als UV

\begin{tabular}{|c|c|c|c|c|c|c|c|c|}
\hline & \multicolumn{2}{|c|}{ 1. Achse } & \multicolumn{2}{|c|}{ 2. Achse } & \multicolumn{2}{|c|}{ 3. Achse } & \multicolumn{2}{|c|}{ 4. Achse } \\
\hline & Eig & VA (\%) & Eig & $\mathrm{VA}(\%)$ & Eig & $\mathrm{VA}(\%)$ & Eig & $\mathrm{VA}(\%)$ \\
\hline$\overline{\mathrm{DCA}}$ & 0,135 & 14,9 & 0,072 & 7,9 & 0,052 & 5,8 & 0,044 & 4,8 \\
\hline Grad.lg. & \multicolumn{2}{|c|}{1,573} & \multicolumn{2}{|c|}{1,241} & \multicolumn{2}{|c|}{1,124} & \multicolumn{2}{|c|}{1,187} \\
\hline PCA & \multicolumn{2}{|l|}{0,275} & \multicolumn{2}{|l|}{0,124} & \multicolumn{2}{|l|}{0,083} & \multicolumn{2}{|l|}{0,067} \\
\hline $\mathrm{RDA}_{20}$ & 0,175 & 45,5 & 0,050 & 13,1 & 0,044 & 11,4 & 0,024 & 6,2 \\
\hline Mte Carlo & \multicolumn{2}{|c|}{$14,9 * * *$} & \multicolumn{6}{|c|}{$2,19 * * *$} \\
\hline $\mathrm{RDA}_{9}$ & 0,165 & 55,2 & 0,039 & 13,2 & 0,036 & 12,1 & 0,017 & 5,8 \\
\hline Mte Carlo & \multicolumn{2}{|c|}{$15,99 * * *$} & \multicolumn{6}{|c|}{$3,83 * * *$} \\
\hline $\mathrm{RDA}_{\mathrm{XY}}$ & 0,097 & 82,1 & 0,021 & 17,9 & 0,215 & 0 & 0,11 & 0 \\
\hline Mte Carlo & \multicolumn{2}{|c|}{$9,42 * * *$} & & & \multicolumn{4}{|c|}{$5,88 * * *$} \\
\hline
\end{tabular}



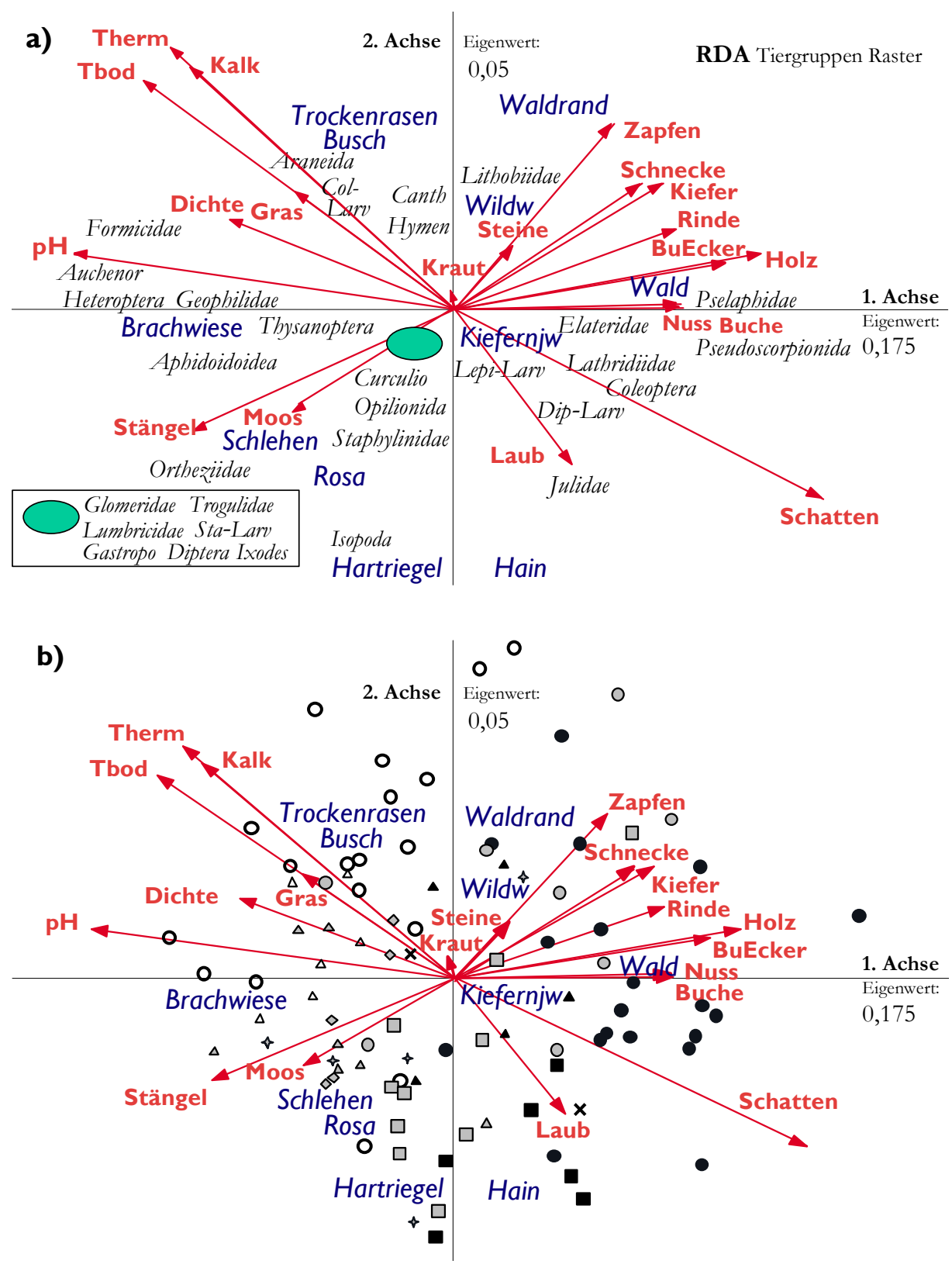

Abb. 12: Ordination der Siedlungsdichte verschiedener Tiertaxa (a) und der Rasterpunkte (b) entlang der beiden ersten Achsen einer Redundanzanalyse (RDA). Die verschiedenen Habitattypen sind als passive Variablen aufgetragen; Legende der Symbole für die Rasterpunkte in Abb. 10; Coleoptera: weitere, überwiegend saprophage Käferfamilien; Elateridae: Schnellkë̈fer-Larven; Col.-Larven: Larven anderer Käferfamilien; Lepi-Larv.: Schmetterlingsraupen; Sta-Larv: Kurzflügelkäfer-Larven; Auchenor: Zikaden; Hymen: Hautflügler obne Ameisen; Canth: Weichkëfer; Wildw: Wildwechsel; Kiefernjw: Jungwuchs Pinus sylvestris. 
Die zweite Achse, die nur einen geringen Anteil der Tiervarianz erklärt, korreliert positiv mit dem Steinanteil, der Menge an Kiefern- und Lärchenzapfen und der Temperatur und negativ mit der Menge an Haselnuss-, Feldahorn- und Weißdornlaub. Dieser Aspekt trennt nun auch Trockenrasen, Einzelbüsche und Waldrand sehr klar von Schlehen-, Rosen- und Hartriegelgebüsch und dem Feldahorn-Hain. Spinnen und die Larven von Weich- und anderen Käfern sind sehr eng mit den warmen und kalkhaltigen Trockenrasenflächen assoziiert, Steinläufer mit dem steinigen und zapfenreichen Waldrand. Die saprophagen Julidae korrelieren sehr eng mit der Menge an Nicht-Buchen-Laub und auch die Isopoda werden mit Hartriegel und Feldahorn zusammen ordiniert.

Saftkugler, Regenwürmer, Kurzflügelkäfer-Larven, adulte Diptera, Schnecken, Brettkanker und Zecken werden durch die ersten beiden Achsen praktisch überhaupt nicht voneinander getrennt; sie tendieren gemeinsam etwas zu den feuchteren Gebüschen hin.

Die hier nicht dargestellte dritte Achse korreliert mit dem Moosgewicht und Steinanteil und trennt den Kiefernjungwuchs und die Rosensträucher vom Feldahornhain und den Schlehdorngebüschen mit den Rüsselkäfern, Asseln, Regenwürmern und Weberknechten. Die Dichte der Ortheziidae korreliert auch hier noch deutlich mit dem Moosgewicht.

Insgesamt tragen der $\mathrm{pH}$-Wert und die Beschattung durch die Vegetation am meisten zur Varianzerklärung der Tiergruppen bei. Viele der Umweltfaktoren korrelieren miteinander, allerdings nur so schwach, dass der „variance inflation factor"،, der starke Multikollinerarität anzeigen würde, für alle Faktoren kleiner als 11 ist. Mit einer automatischen, aufbauenden (forward) Auswahl der Umweltfaktoren wird jeder zunächst allein getestet - die resultierenden kanonischen Eigenwerte sind als marginale Effekte in Tabelle 6 angegeben - um anschließend, beginnend mit dem Faktor, der den größten Varianzanteil erklärt, ein Modell aufzubauen. Die weiteren Faktoren werden jetzt in der Rangfolge ihrer zusätzlich erklärenden Varianz in das Modell mit aufgenommen. Wenn die Umweltfaktoren nicht komplett unabhängig sind, ist dabei die Reihenfolge eine andere als die der marginalen Effekte. Beispielsweise erklärt die maximale Bodentemperatur für sich genommen den dritt-größten Varianzanteil, da sie jedoch negativ mit der Beschattung korreliert (Produkt-Moment-Korrelations-Koeffizient: -0,85), wird sie erst später in das Modell aufgenommen; an dritter Stelle kommt in diesem Falle das Trockengewicht der Stängel (Odermennig, Margerite u.a.), das relativ unabhängig von den beiden anderen, bisher in das Modell aufgenommenen, Faktoren $\mathrm{pH}$ $(0,19)$ und Beschattung $(-0,33)$ ist. Dennoch erklärt das Modell bereits vor Hinzunahme der Stängelmenge $2 / 3$ ihres marginalen Effekts $\left(\lambda_{1}=0,06\right)$, die zusätzliche Eigenwertsteigerung $\left(\lambda_{A}\right)$ beträgt nur noch 0,02 (Tab. 6).

Die konditionalen Effekte („extra-fit-Anteile“) wurden mit einem Permutationstest auf ihre Signifikanz getestet (P, Tab. 6). Bei 20 solcher Tests steigt allerdings die Wahrscheinlichkeit, nur per Zufall signifikante Werte zu finden. Mit einer Bonferroni-Korrektur (sowohl nach Bonferroni-Fisher als auch nach Bonferroni- 
Holm) bleiben nur der $\mathrm{pH}$-Wert und der Beschattungsgrad auf dem $5 \%$ - Niveau signifikant.

Tabelle 6: Effekte der Umweltvariablen auf die Ordination der Tiergruppen jeweils einzeln (marginale Effekte mit den Eigenwerten $\lambda_{1}$ ) und als zusätzlicher Effekt zu den bereits in das Modell eingerechneten Faktoren (konditionale Effekte; mit zusätzlichem (extra-fit) kanonischem Eigenwert $\lambda_{A}$, F-Wert eines Monte-Carlo Permutationstest auf diesen zusätzlichen Effekt, Feblerwahrscheinlichkeit und Reihenfolge der Hinzunahme der Variablen in das Modell), berechnet mit automated forward selection. Abkürzungen: Kapitel 4.2.

\begin{tabular}{|c|c|c|c|c|c|}
\hline \multirow[b]{3}{*}{ Variable } & \multicolumn{5}{|c|}{ Effekte der Umweltfaktoren (automated forward selection) } \\
\hline & \multirow{2}{*}{$\begin{array}{c}\text { Marginale Ef- } \\
\text { fekte }\end{array}$} & \multicolumn{4}{|c|}{ Konditionale Effekte } \\
\hline & & LambdaA & $\mathrm{F}$ & $\mathrm{P}$ & Rang \\
\hline $\mathrm{PH}$ & 0,11 & 0,11 & 10,86 & 0,001 & 1 \\
\hline Schatten & 0,11 & 0,05 & 4,92 & 0,001 & 2 \\
\hline Tbod & 0,08 & 0,01 & 0,66 & 0,814 & 17 \\
\hline Therm & 0,08 & 0,02 & 2,12 & 0,015 & 7 \\
\hline Holz & 0,07 & 0 & 0,5 & 0,947 & 20 \\
\hline Kalk & 0,07 & 0,01 & 0,99 & 0,447 & 12 \\
\hline BuEcker & 0,06 & 0,01 & 0,91 & 0,52 & 14 \\
\hline Stängel & 0,06 & 0,02 & 2,85 & 0,006 & 3 \\
\hline Dichte & 0,05 & 0,02 & 2,07 & 0,021 & 6 \\
\hline Buche & 0,05 & 0,01 & 1,44 & 0,116 & 11 \\
\hline Nuss & 0,04 & 0,01 & 1,94 & 0,031 & 8 \\
\hline Kiefer & 0,04 & 0,01 & 1,56 & 0,103 & 10 \\
\hline Rinde & 0,04 & 0,01 & 0,89 & 0,552 & 15 \\
\hline Moos & 0,04 & 0,03 & 2,71 & 0,003 & 4 \\
\hline Schnecke & 0,03 & 0 & 0,73 & 0,741 & 18 \\
\hline Zapfen & 0,03 & 0,01 & 0,94 & 0,494 & 13 \\
\hline Gras & 0,03 & 0 & 0,75 & 0,719 & 16 \\
\hline Laub & 0,02 & 0,02 & 1,87 & 0,039 & 9 \\
\hline Steine & 0,02 & 0,02 & 2,27 & 0,018 & 5 \\
\hline Kraut & 0 & 0,01 & 0,59 & 0,856 & 19 \\
\hline
\end{tabular}

Werden die 11 deutlich nicht signifikanten Umweltvariablen aus dem Modell herausgenommen, sinkt der Eigenwert der ersten Achse auf 0,165 (RDA, Tab. 5), also erklärt sie nur 1\% der Artenvarianz weniger als diejenige des Modells mit allen 20 Faktoren. Auch die Ordination bleibt weitgehend identisch und wird deshalb nicht dargestellt. 


\subsubsection{Räumliche Verteilung der Tiergruppen}

Da nur für die bodennahen Straten systematisch Daten aufgenommen wurden, beschränkt sich die Verteilungsanalyse auf zwei Dimensionen, so dass hier im Prinzip nur die flächenhafte Verteilung betrachtet werden kann.

In einem ersten Schritt wurden nur die X-Y-Koordinaten jedes Rasterpunktes als erklärende Umweltvariablen in der Redundanzanalyse berücksichtigt, so dass jetzt allein die relative räumliche Lage die Tiergruppengradienten erklären kann. Es werden immer noch $11,8 \%$ der Tiervarianz durch die beiden ersten Achsen erklärt $\left(\mathrm{RDA}_{\mathrm{xy}}\right.$, Tab. 5), etwas mehr als die Hälfte der Anteile des Modells mit 20 Umweltvariablen und ohne die Koordinatenwerte. Die erste Achse korreliert eng mit den X-Werten, also dem Ost-West-Gradienten und zeichnet praktisch den Hangverlauf nach. Der Nord-Süd-Gradient (Y-Werte, eng korreliert mit der 2. Achse) erklärt nur noch $2 \%$ der Varianz der Siedlungsdichten der Tiergruppen.

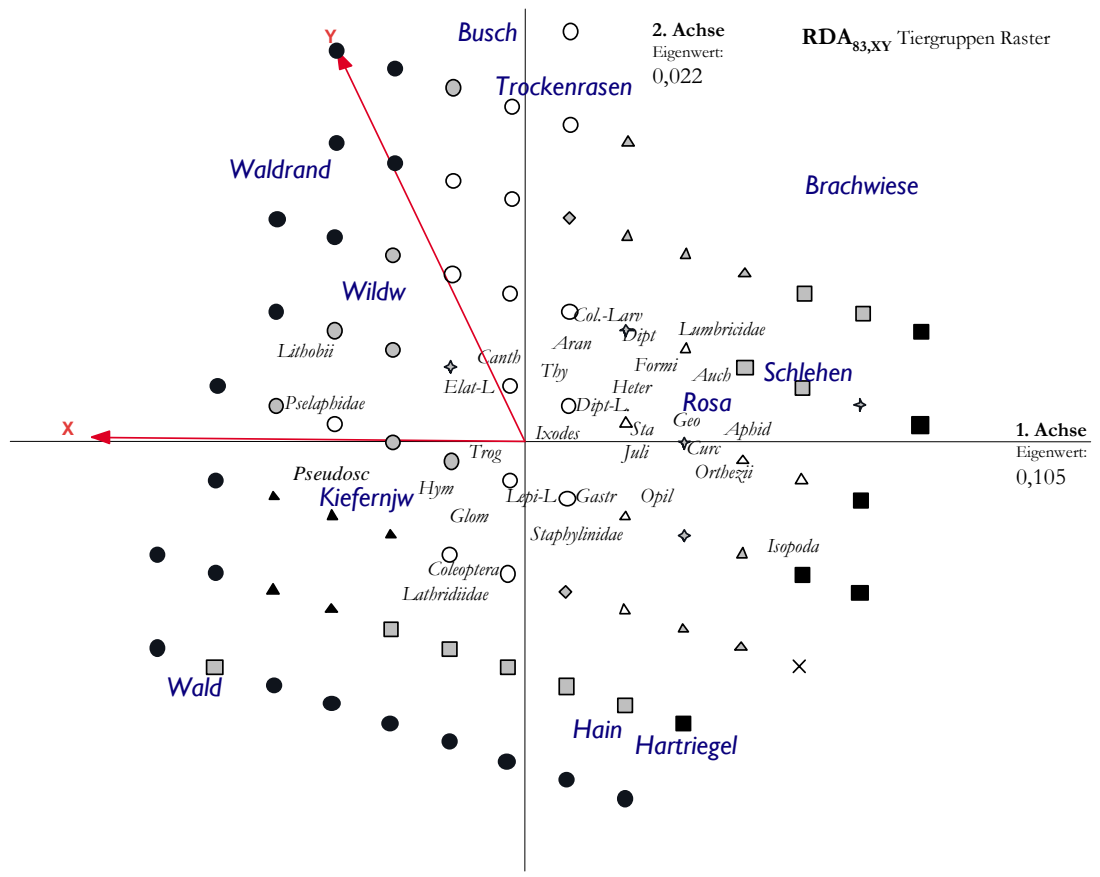

$A b b$. 13: Ordination der Siedlungsdicbte verscbiedener Tiertaxa und der Rasterpunkte zusammen mit den X-und Y-Koordinaten als erklärende Faktoren entlang der beiden ersten Acbsen einer Redundanzanalyse $\left(\mathrm{RD} A_{83, x y}\right)$. Die verscbiedenen Habitattypen sind als passive Variablen aufgetragen (Abkürzungen wie bei Abb.12); Legende der Symbole für die Rasterpunkte in Abb. 10; ausgeschriebene Namen der ordinierten Tiergruppen ergeben sich aus Abb. 12, nähere Erläuterungen im Text. 
Mit beiden Achsen sind die Umweltvariablen komplett erklärt (100 \% der ArtenUmwelt-Relation), so dass die dritte Achse jetzt den größtmöglichen Gradienten der verbliebenen Varianz abbilden kann und ähnlich wie die erste Achse der PCA, vor allem zwischen den Ameisen und Schnabelkerfen und den „Waldtieren“ Pseudoskorpione und saprophage Käfer trennt.

Nur für das Kernraster, also ohne den Transekt auf Fläche 1a, steigt der Eigenwert der ersten Achse um ein Prozent (Tab. 8), die erste Achse ist dann fast mit der X-Achse identisch (Abb. 13). Aus der Ordination der Tiergruppen werden trotz der Abweichung der Y-Achse von der 2. Achse auch die Tendenzen für lineare Trend-Oberflächen ersichtlich: eine zweidimensionale Regressionsfläche würde vom Ursprung der RDA-Achsen zu den Tiergruppen-Punkten hin ansteigen, da der Vektor für diese Punkte nach den kleinsten Abweichungsquadraten („,best fit") errechnet wurde.

Beispielsweise verläuft die lineare Trend-Oberfläche für die Spinnen-Siedlungsdichte von der Süd-Ost-Ecke zur Nord-West-Ecke des Rasters hin ansteigend. Die höchsten Dichten erreichen die Spinnen (im Mittel) auf der Glatthaferwiese vorm Feldahorn-Hain mit 330 Ind./ $\mathrm{m}^{2}$. Auch ein Vektor vom Ursprung der RDA-Achsen zum Spinnen-Ordinationspunkt zeigt in diese Richtung.

Die räumlichen Trends für alle Tiergruppen gemeinsam werden deutlich, wenn die Werte der ersten Hauptkomponente der Tiergemeinschaft (Abb. 11) für jeden Rasterpunkt entsprechend der Lage auf der Fläche dargestellt werden (Abb. 14).

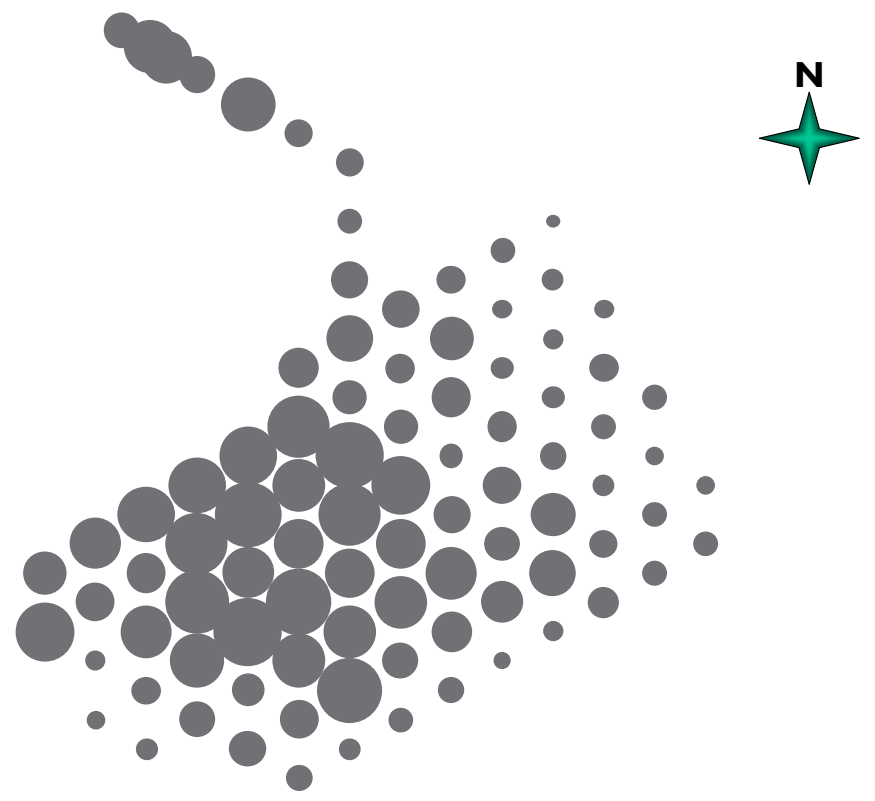

Abb. 14: Verteilung der Hauptkomponenten-Werte der Rasterpunkte einer PCA der Siedlungsdichten der Tiergruppen auf der Fläche: Die Kreisfläche ist dem Achsenwert proportional. 
Es wird deutlich, dass alle Rasterpunkte mit hohen Achsenwerten im Zentrum der Fläche und am Ende des Transekts liegen: auf der Glatthaferwiese und in ihren Übergangsbereichen zum Halbtrockenrasen bzw. in die Gebüsche. Die Tiergemeinschaft bildet in dieser Form ziemlich exakt die Vegetationsgegebenheiten ab. Innerhalb gleicher Habitattypen gibt es dabei nur geringe Schwankungen.

Auch die abiotischen Umweltbedingungen geben diesen zweidimensionalen Gradienten wieder; die einzelnen Habitatzonen werden aber entweder weniger deutlich getrennt, wie beim pH-Wert, der über den ganzen Hang hinweg gleichmäßig ansteigt und wieder abfällt (Abb. 15 a), oder sie werden deutlich kleinräumiger unterschieden als durch die Tiergemeinschaft, so wie beispielsweise durch den Beschattungsgrad (Abb.15d). Grundsätzlich trennen aber auch die abiotischen Variablen immer besonders deutlich die Wald- von den Wiesenhabitaten.
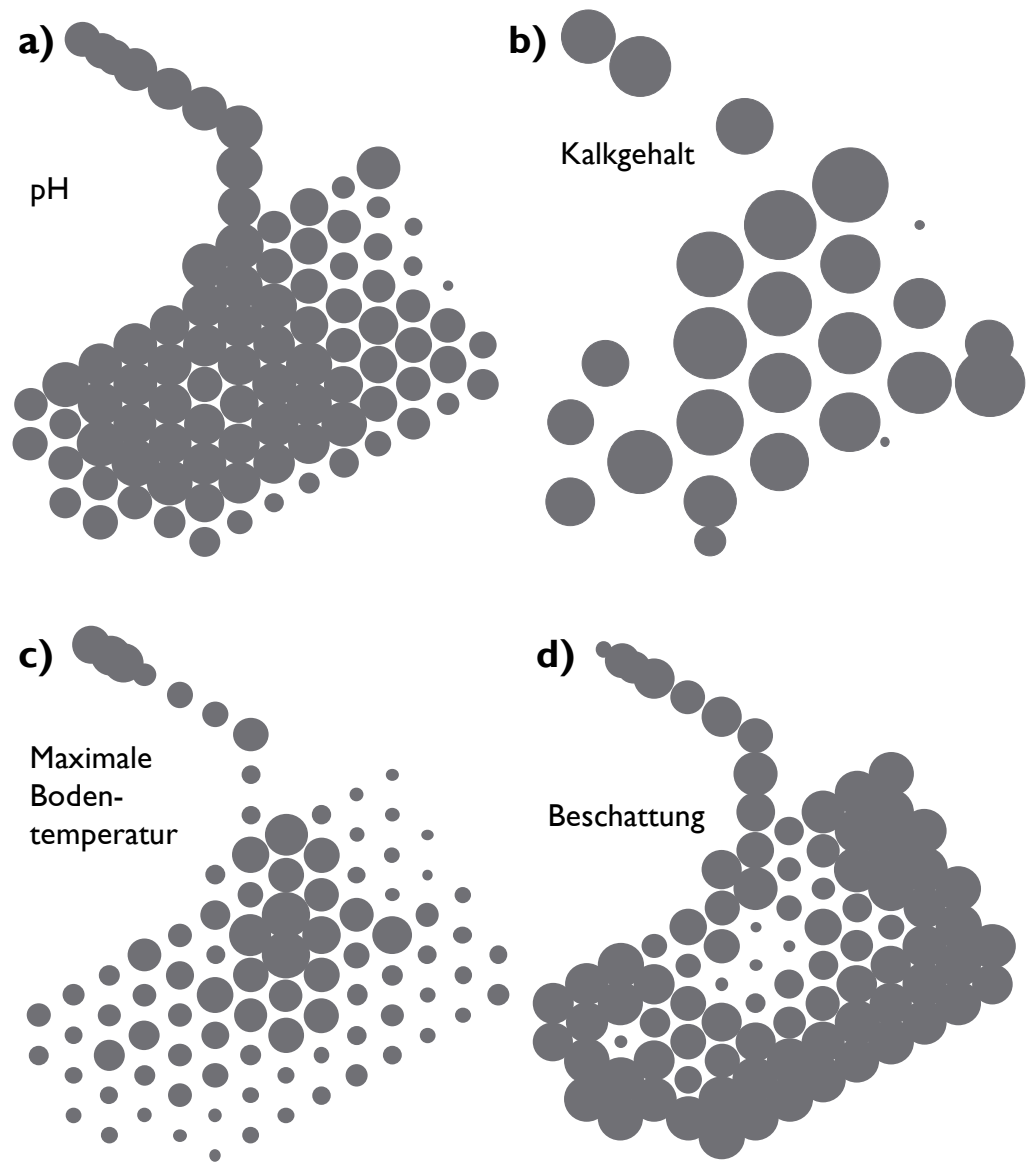

Abb. 15: Abiotische Umweltbedingungen auf der Untersuchungsfläche: $p H$-Wert (a), Kalkgehalt (b), Maximale Bodentemperatur (c) und Beschattungsgrad (d); die Fläche der einzelnen Kreise entspricht den relativen Verbältnissen der Absolutwerte zueinander. 
Dieser Hauptgradient zwischen Wald und Offenland ist gleichermaßen deutlich bei der räumlichen Verteilung der einzelnen Tiergruppen aus unterschiedlichen Gilden erkennbar. Innerhalb jeder Gilde mit verschiedenen Ernährungsformen gibt es unterschiedliche Verteilungen, aber auch gemeinsame Tendenzen. Regenwürmer waren auffallend gleichmäßig über die gesamte Fläche verteilt (Abb. 16 a) und erreichten ihre höchsten Dichten im unteren Hain. Schnurfüßer und Asseln waren auf den Trockenrasen-Flächen nur vereinzelt in niedrigen Dichten vertreten, sie waren vor allem am Unterhang in Hain und Schlehengebüsch zu finden. Die Moderkäfer (Abb. 16 d) kamen in geringen Siedlungsdichten vor allem im Wald und Hartriegel-Hasel-Gürtel vor. Insgesamt waren in den waldartigen Lebensraumtypen die Dichten saprophager Tiere deutlich höher als auf den offenen Flächen. Dabei wurden tiefgründigere Böden und das Laub von Feldahorn, Hasel und Weißdorn gegenüber dem von Buche bevorzugt.
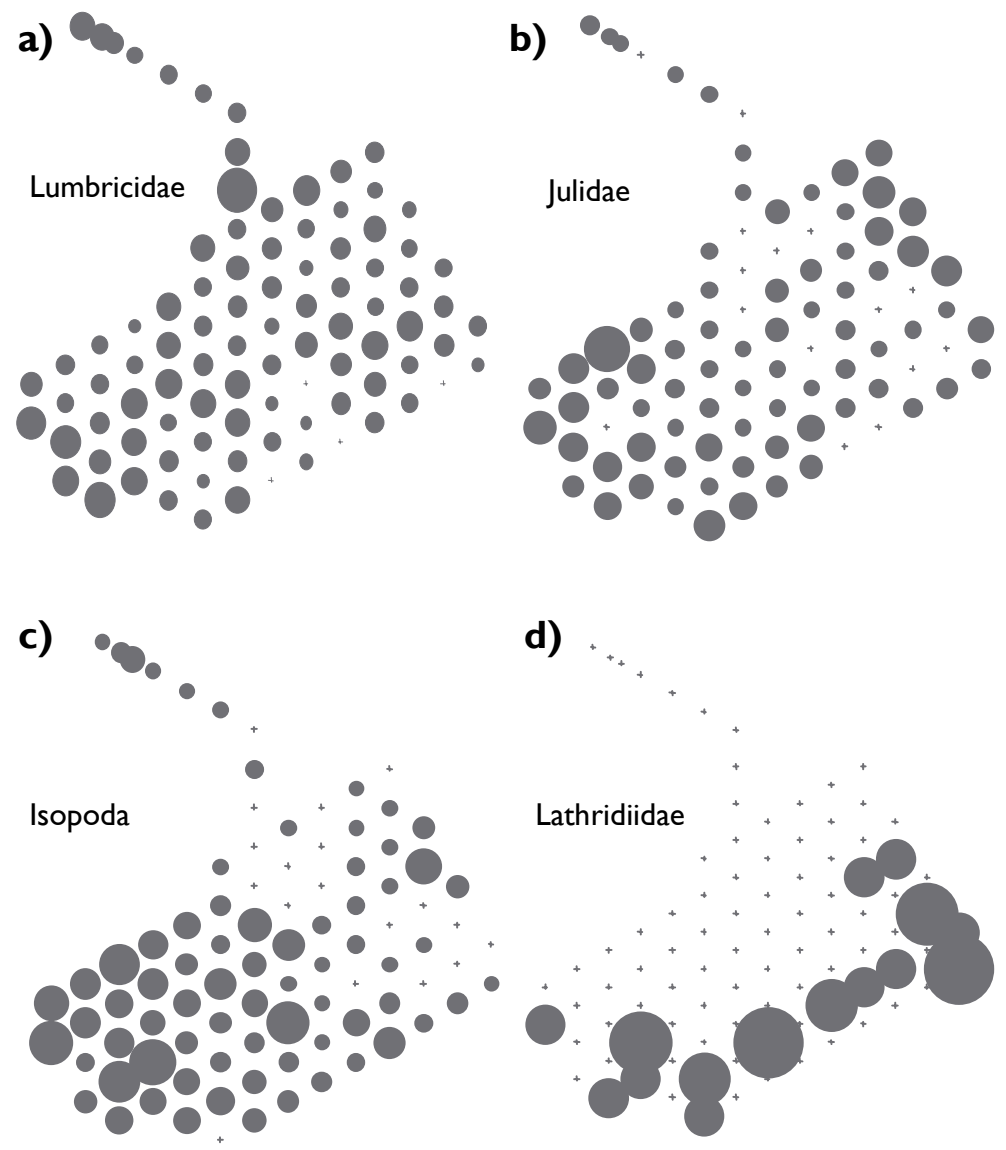

$A b b$. 16: Siedlungsdicbten der saprophagen Tiere auf der Untersucbungsfläche: Regenwürmer (a), Schnurfüßer (b), Asseln (c) und Moderkäfer (d); die Fläche der einzelnen Kreise entspricht den relativen Verbältnissen der Absolutwerte zueinander. 

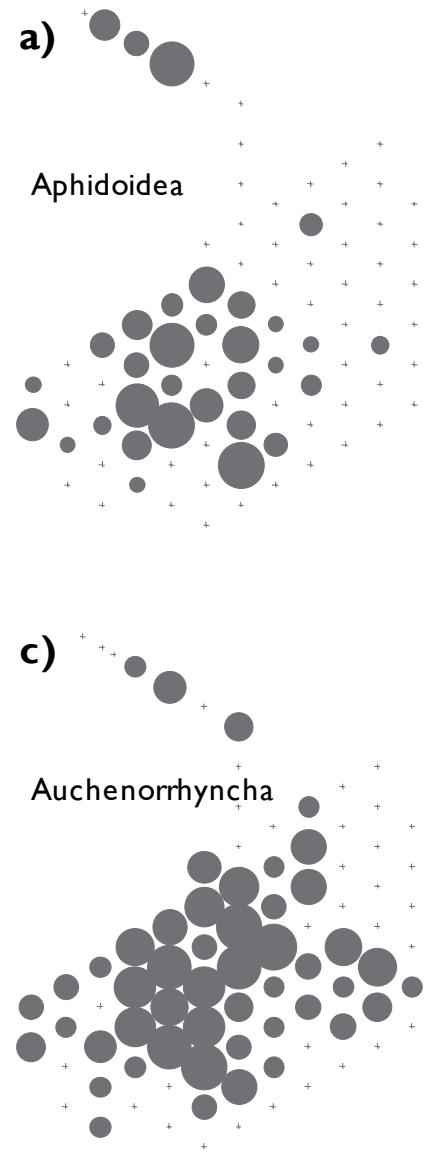
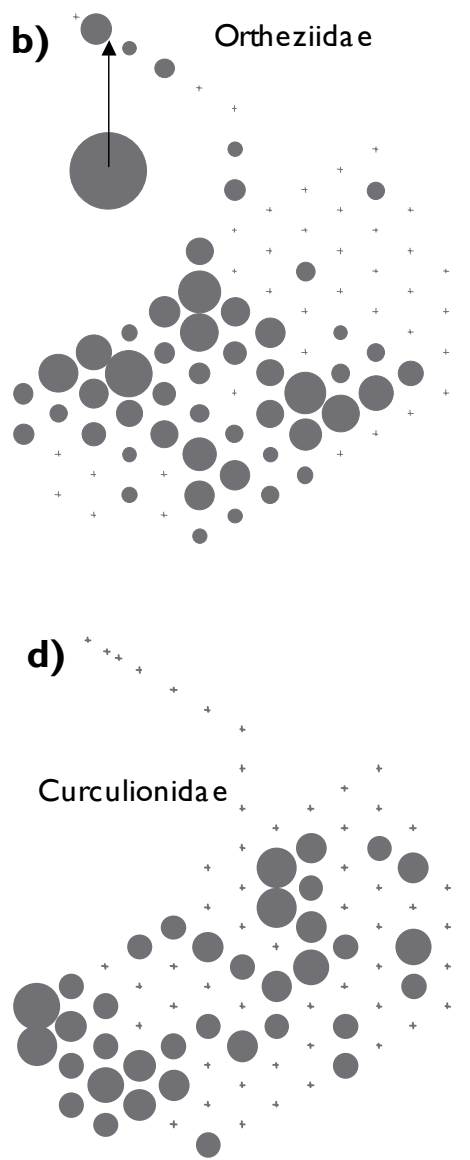

Abb. 17: Siedlungsdicbten der phytophagen Tiere auf der Untersuchungsfläche: Blattläuse (a), Röbrenscbildläuse (b), Zikaden (c) und Rüsselkäfer (d); die Fläche der einzelnen Kreise entspricht den relativen Verbältnissen der Absolutwerte zueinander.

Thysanoptera wurden nur in den Bodenproben eines schmalen Übergangsbereichs zwischen Glatthaferwiese und Halbtrockenrasen nachgewiesen. Pflanzenläuse und Zikaden wurden oberhalb dieses Übergangsbereiches, auf dem Trockenrasen und im Wald, ebenfalls nicht gefunden, unterhalb auf der Brachwiese hingegen sehr konstant und häufig. Die Zikaden hatten einen ganz deutlichen Schwerpunkt ihrer Siedlungsdichten in der Mitte der Fläche (Abb. 17 c), die Röhrenschildläuse erreichten auch in den umliegenden Gebüschen höhere Abundanzen. Die Blattläuse siedelten etwas fleckenhafter, ähnlich wie die Ameisen, über die Fläche verteilt. Allerdings waren die Ameisen in allen Bodenproben vertreten, nur ihre Individuenzahl schwankte beträchtlich. Auch andere Prädatoren, wie die Spinnen und Steinläufer, kamen auf der ganzen Fläche in fast allen Proben vor, hatten aber ebenfalls in spezifischen Bereichen deutlich höhere Dichten (Abb. 18 a, d). Die Dichte der Spinnen und Ameisen korreliert dabei deutlich mit den Achsenwerten 
der 1. Hauptkomponente (Abb. 14). Beide Tiergruppen erreichten ihre höchsten Dichten im Bereich der Brachwiese, auf Teilen des Trockenrasens und an wärmebegünstigten Wald- und Gebüschsäumen. In den Gebüschen nimmt die Spinnendichte ab, während in einigen Bereichen die Ameisen auch hier höhere Dichten erreichen. Die Kurzflügelkäfer haben gerade in solchen verbuschten Bereichen höhere Individuendichten als in den voll besonnten Bereichen; sie scheinen sogar den offenen Trockenrasenbereich komplett zu meiden.
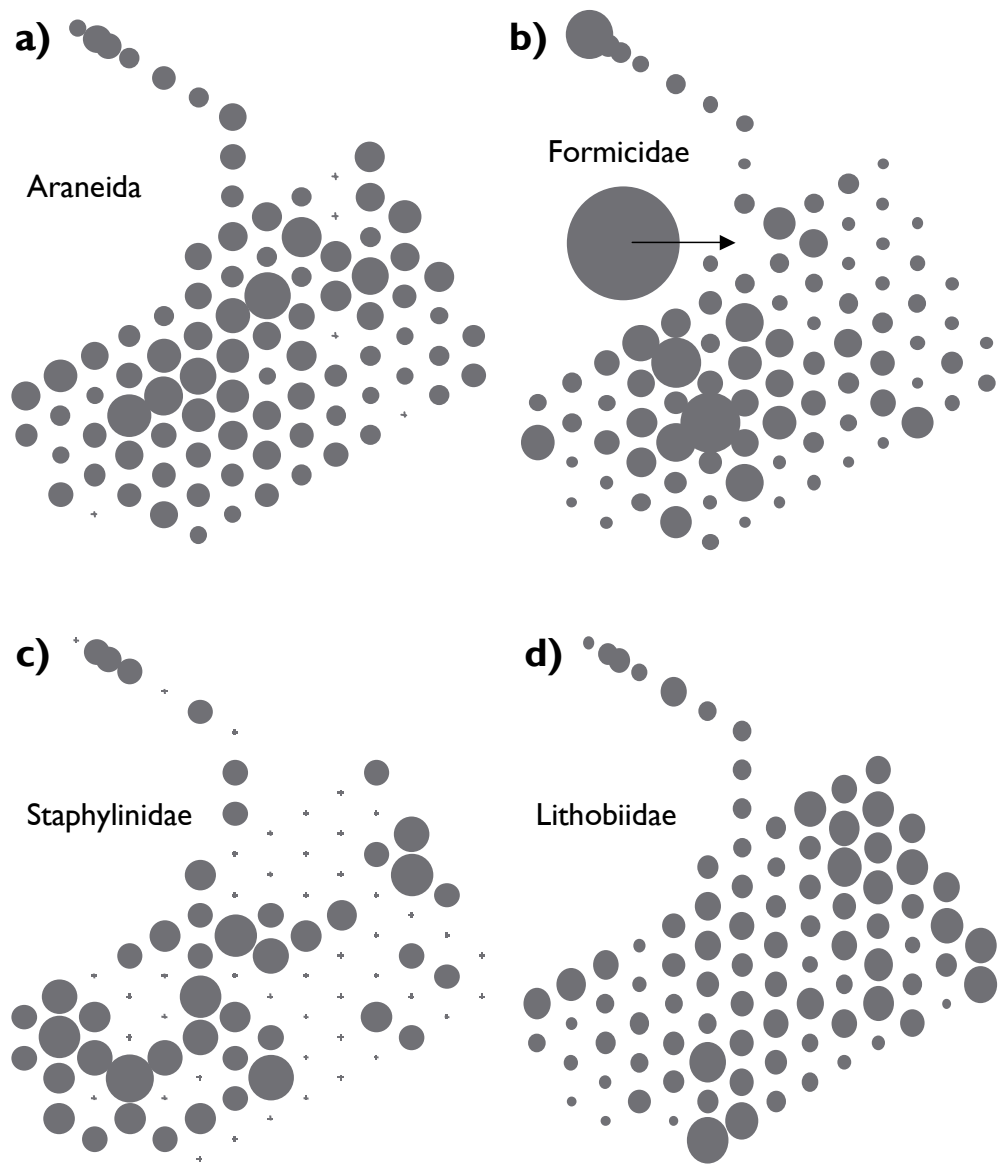

Abb. 18: Siedlungsdichten der zoophagen Tiere auf der Untersuchungsfläche: Spinnen (a), Ameisen (b), Kürafliügelkäfer (c) und Steinläufer (d); die Fläche der einzelnen Kreise entspricht den relativen Verbältnissen der Absolutwerte zueinander.

Die Steinläufer waren wie die Ameisen in jeder Probe zu finden, doch hatten sie im Unterschied zu den anderen häufigen Zoophagen im Wald viel höhere Dichten. Nur im Bereich der Haselstrauch-Reihe auf dem Steinwall, der am Oberhang im Wald die südliche Grenze der Wiese 1 bildet, hatten diese Hundertfüßer, wie auch die Erdläufer, sehr geringe Dichten. Die Geophilidae waren in allen Waldbe- 
reichen nur vereinzelt vertreten; sie kamen vorwiegend auf der Brachwiese und in den wärmeren Schlehengebüschen häufiger vor. Die für die Waldbereiche kennzeichnenden Zoophagen sind, wenn auch nur in geringen Gesamtdichten vorkommend, die Pseudoscorpionida und Pselaphidae; alle Individuen dieser Taxa, bis auf einen Palpenkäfer in einem Nest von Lasius alienus, wurden nur in den Wald- und dichten Gebüschzonen gefunden.

\subsubsection{Verteilung der Arten in Abhängigkeit von der Umwelt}

\section{Pflanzen}

Die Pflanzenarten in der Übergangszone vom Halbtrockenrasen zur Glatthaferwiese bilden einen deutlichen Gradienten aus, die Eigenwerte einer „detrended“ Korrelationsanalyse (DCA) betragen für die erste Achse 0,40 und für die zweite Achse 0,10. Die erste Achse erklärt $45 \%$ der Varianz der Deckungsgrade der einzelnen Arten. Die Hangzonen werden vom oberen Bereich her nacheinander in der gleichen Reihenfolge ordiniert, die ihrer räumlichen Lage entspricht; nur die unterste von 5 Zonen wird intermediär ordiniert, da hier einige Arten vorkommen, die sonst nur in den beiden oberen Abschnitten wachsen (vgl. Tab. 1, Kap. 2.3.). Der obere Teil wird stärker von Moosen, Kleinem Odermennig, ZypressenWolfsmilch, Großem Wiesenknopf, Ackerkratzdistel, Gemeinem Hornklee, Gemeiner Quecke, Aufrechter Trespe und dem Gemeinen Rispengras geprägt, der mittlere Bereich von Wiesen-Margerite und Wirbeldost und der untere Abschnitt von Wiesenlabkraut, Gemeiner Flockenblume, Tüpfel-Johanniskraut, Wiesenkerbel, Tragant, Fieder-Zwenke und Glatthafer. Im unteren Fünftel wird der Odermennig auch wieder vorherrschend; der Hopfenklee ist in allen Bereichen dominant.

Den höchsten Erklärungswert für die Varianz der Pflanzenarten haben einerseits der Steinanteil und die Luftfeuchte, die in diesem Bereich nach unten hin zunehmen, wobei der Steinanteil im unteren Fünftel wieder ansteigt, sowie andererseits die Beschattung und Streubedeckung im Frühjahr, die in der Mitte am geringsten sind. Moosgewicht, Bodendichte und Temperatur im Boden und in der Krautschicht, die nach unten hin geringer werden, und der Wassergehalt des Bodens, der parallel dazu zunimmt, sowie der in der Mitte ansteigende Kalkgehalt bzw. der dort leicht abnehmende $\mathrm{pH}$-Wert korrelieren jeweils stark mit einem der anderen Faktoren.

Im Frühjahr ist die Vegetation stärker durch Gräser geprägt, im Spätsommer überwiegen dann die Kräuter. Nur in Bereichen mit hohen Anteilen an Glatthafer und Fiederzwenke bleibt noch eine größere Menge an oberirdischer Grasbiomasse erhalten, die zu Teil einen dichten Filz aus vertrockneter Streu bildet, der nicht direkt dem Boden aufliegt und somit auch wesentlich länger der Zersetzung widersteht. 


\section{Zikaden}

In einer DCA der Siedlungsdichten der Zikaden auf einem Teil der Brachwiese (vgl. Kap. 7 und Rachor 2004) werden die Zentroide der Hangabschnitte sehr ähnlich zu ihrer relativen tatsächlichen Lage im Raum ordiniert, was auf eine starke räumliche Autokorrelation der Zikadengemeinschaft deutet. Ein starker Abfall der Eigenwerte der extrahierten Achsen durch Hinzunahme der Umweltvariablen Temperatur, Wassergehalt und oberirdischer Biomassen der Gräser, Kräuter und Moose in die Ordination zeigt einen schlechten Erklärungswert dieser Faktoren für die Varianz der Zikadenarten an (Tab. 7).

Hangabwärts - mit zunehmender Vegetationsdichte - wurden mehr Zikadenarten mit einer hohen Anzahl von Individuen gefunden, entsprechend trennt die erste Achse, die v.a. mit der Grasbiomasse korreliert, zusammen mit der zweiten Achse, die eng mit der Bodentemperatur und der Moosbiomasse korreliert, deutlich die Trockenrasenparzellen mit den Arten Arboridia parvula und Anaceratagallia venosa von den übrigen Parzellen und den übrigen Arten. Das andere Ende dieses Gradienten wird von den Parzellen des 4. Blocks (vgl. Tab. 1), die sehr eng beieinander ordiniert werden, geprägt.

\section{Spinnen}

Die Varianz von 30 Spinnenarten auf dem gleichen Teil der Brachwiese kann durch die erste Achse einer DCA nur zu 14,4\% erklärt werden, diese Achse hat einem Eigenwert von knapp 0,2 (Sanders 2004). In der kanonischen Ordination mit den gleichen Umweltvariablen wie bei der Zikaden-Ordination sinkt dieser Wert auf 0,15; die erste Achse der CCA erklärt noch 10,9\% der Artenvarianz und 38,9 \% der Arten-Umwelt-Relation (Tab. 7). Durch die erste Achse wird trotz des geringen Erklärungswerts ein sehr markanter Unterschied deutlich: alle Netzspinnenarten, die in dichter Vegetation deutlich häufiger waren, werden durch diese Achse von fast allen vaganten Spinnenarten getrennt. Gleichzeitig werden die Halbtrockenrasen-Bereiche mit hoher Bodentemperatur und Moosbiomasse deutlich von den Brachwiesen-Bereichen mit hoher Vegetationsdichte getrennt. Einzige Ausnahmen sind die Tapezierspinne Atypus piceus, die mit ihrem Fangschlauch ja auch ein Netz zum Beutefang nutzt, und die Kammspinne Zora sylvestris Kulczynski. Atypus ist zusammen mit mehreren Zelotes-Arten eine „Charakterart“ der offenen Vegetation, Zora mit Kugel- und Kieferspinnen eher typisch für eine geschlossene Vegetationsdecke.

Die zweite Achse korreliert mit dem Wassergehalt des Bodens und der Grasbiomasse. Sie trennt bei den vaganten Spinnen beispielsweise zwischen den Springspinnen Euophrys aequipes O.P. Cambridge in feuchteren Bereichen und Evarcha falcata Clerck in trockneren Bereichen mit weniger Gras. Bei den Netzspinnen trennt dieser Gradient zwischen der Radnetzspinne Mangora acalypha Walckenaer und den juvenilen Netzspinnen in Flächen mit viel Gras einerseits und verschiedenen adulten Baldachinnetz- und Kugelspinnen, wie z.B. zwei Tenuiphantes-Arten, in trockeneren Bereichen andererseits. 


\section{Ameisen}

Auf Artniveau zeigen die Ameisen sehr klare Unterschiede zwischen den verschiedenen Bereichen der untersuchten Fläche. Da es einige Proben gibt, in denen sehr viele Individuen der gleichen Art gefunden wurden, im Extremfall ein Nest von Solenopsis fugax im Wacholder-Orchideen-Bereich des Halbtrockenrasens mit 2035 Arbeiterinnen, 30 Larven und 1 Königin (A5 an der „Abzweigung“ des „Seitenarms“, vgl. Abb. 18 b), wurden die Individuenzahlen vor der Datenanalyse logarithmiert. Um Arten, die nur an wenigen Punkten gefunden wurden, nicht überzubewerten, wurden seltene Arten geringer gewichtet („downweighting of rare species“, ter Braak \& Smilauer 2002). Die Eigenwerte einer DCA für die Ameisen-Individuendichten im Mai waren mit 0,68 für die erste Achse und 0,56 für die zweite Achse vergleichsweise sehr hoch (Tab. 7). Auch bei einer Ordination der Ameisendichten im September von der gleichen Teilfläche der Brachwiese wie die analysierten Zikaden- und Spinnendaten bestätigt der Eigenwert der ersten Achse von 0,48 mit $40 \%$ erklärter Varianz die Güte des erfassten Gradienten. Bei Gradientenlängen von 3,6 bzw. 2,7 im Frühjahr und September ist für die Ameisenarten die Auswertung mit unimodalen Modellen sinnvoll.

Die erfassten Umweltfaktoren im September konnten diese deutlichen Unterschiede in der Ameisengemeinschaft allerdings nicht gut erklären, die Eigenwerte der ersten beiden Achsen sinken in der kanonischen Korrespondenzanalyse (CCA) auf 0,13 und 0,085.

Für den gesamtem Gradienten konnten die Umwelt-Daten die Tierdaten aus dem Frühjahr wesentlich besser erklären: der Eigenwert der ersten Achse sinkt von 0,68 auf 0,56, derjenige der zweiten von 0,56 auf 0,41 (Tab 7 b).

Auch für die Ameisenarten trennt die erste Achse deutlich den Wald von der Wiese. Am deutlichsten ist diese Trennung zwischen Buchenwald und Brachwiese, also Probenahmepunkten im Wald mit hohem Anteil an Buchenblättern und den Bereichen der Brachwiese mit hoher Bodentemperatur. Dazwischen werden die Schwerpunkte der Rasterpunkte unter Rosensträuchern, im Trockenrasen-Bereich, im Schlehengebüsch, unter einzelnen Bäumen oder Büschen und am Waldrand eingeordnet (Abb. 19).

Die zweite Achse korreliert mit Dichte, Kalkgehalt, Temperatur in der Krautschicht und dem Steinanteil im Boden. Insbesondere die Arten der Gattung Leptothorax werden durch die Ordination über einen großen Bereich verteilt, der ihr tatsächliches Vorkommen auf der Fläche gut wiedergibt: L. nylanderi wurde nur im Wald gefunden; L. parvulus in etwas lichteren Waldbereichen und am Waldrand, L. tuberointerruptus an waldrandnahen Halbtrockenrasen-Stellen und L. interruptus im Übergangsbereich von der Glatthaferwiese zum Halbtrockenrasen. Lasius flavus und Lasius alienus haben, auf die erste und zweite Achse bezogen, fast identische Verteilungsmuster und Lebensraum-Ansprüche. Zusammen mit Myrmica sabuleti stellen sie die wichtigsten Ameisenarten auf der Untersuchungsfläche dar. Alle drei Arten sind eher mit der Brachwiese korreliert. Für den Trockenrasen sind die „typischen“ Arten am ehesten Ponera coarctata, Tetramorium caespitum und Solenopsis fugax. 
Tabelle 7: Eigenwerte und Varianzaufklärungen (VA) der ersten vier Achsen je einer ,detrended" (DCA) und einer kanonischen Korrespondenzanalyse (CCA) der Individuenzablen der Tierarten aus Saugfängen (Auchenorrbyncha) bzw. Bodenproben (Araneida, Formicidae) von den offenen Bereichen auf Wiese 1 im September 2002 (a) bzw. vom Wald-Wiese-Gradienten im Mai 1998 (b). F-Werte und Signifikeanzniveaus je eines Monte-Carlo-Tests mit 999 nicht-restriketiven Permutationen ((*): $p<0,1 ; *: p<0.05 ;{ }^{* *}: p<0,01$; *** $\left.p=0,001\right)$.

a)

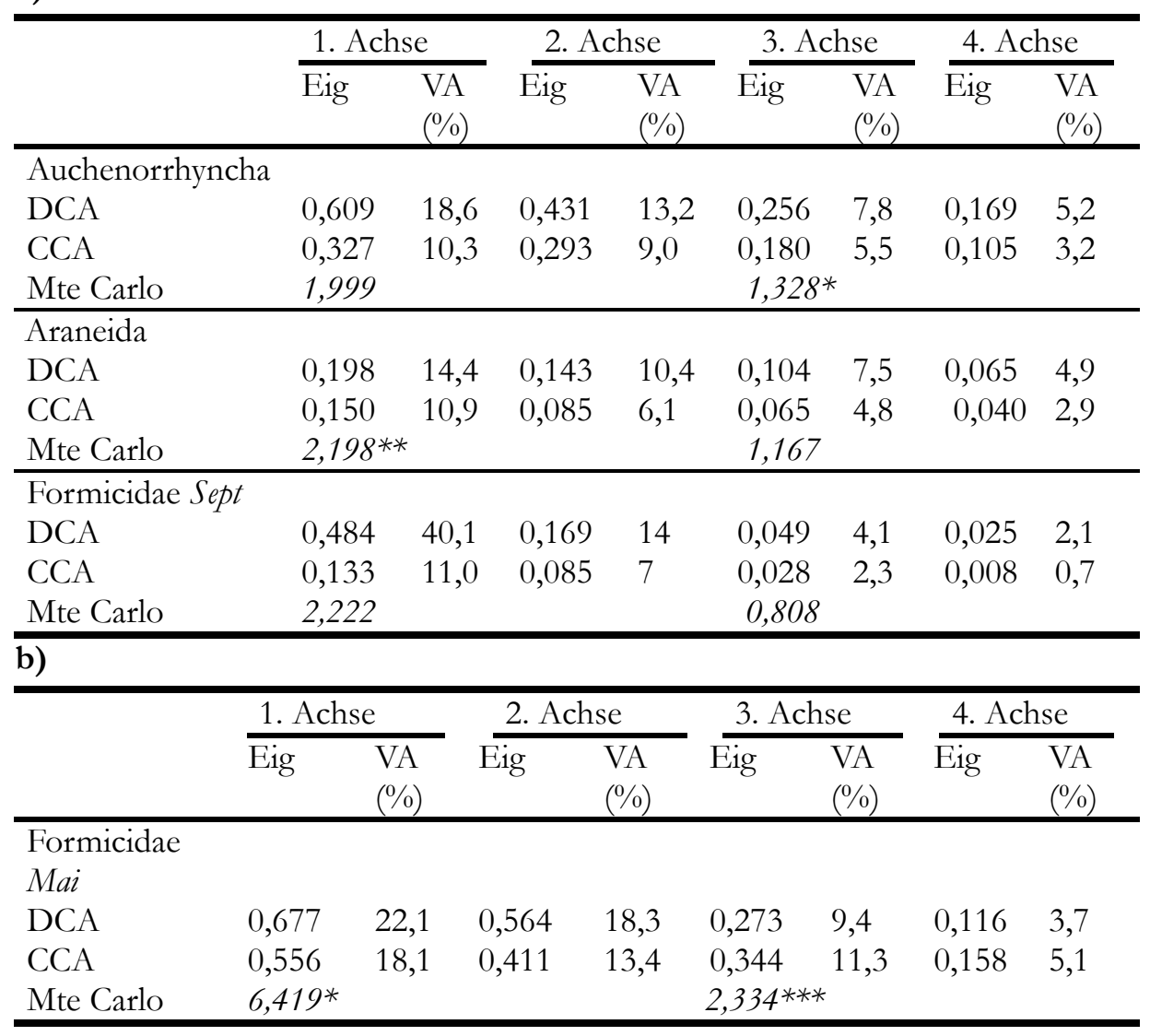




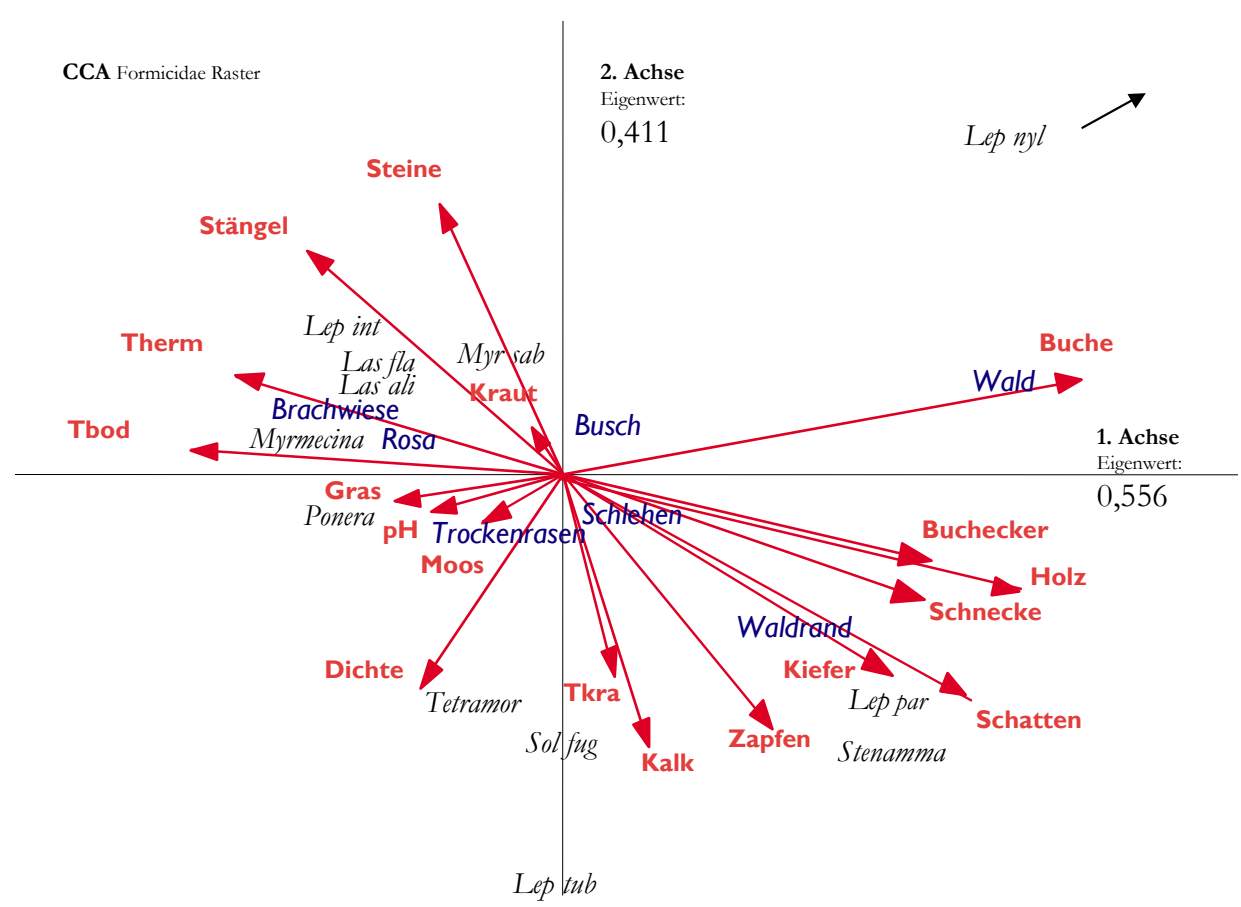

Abb. 19: Ordination der beiden ersten Achsen einer Kanonischen Korrespondenzanalyse (CCA) für die Arbeiterinnendichten der Ameisenarten im Mai 1998. Die verschiedenen Habitattypen sind als passive Variablen aufgetragen; Lep = Leptothorax, Las = Lasius, Myr= Myrmica, Sol= Solenopsis; Tkra = Mittlere Temperatur in der Krautschicht; Artnamen: Tabelle 4, übrige Umweltvariablen: Kap. 4.2. 


\subsection{Diskussion}

\subsubsection{Diversität der Ameisenfauna}

\section{Erfassungsmethoden}

Ein Vergleich von Saugfängen aus einem aufgesetztem Stechzylinder mit der Hitzeextraktion der anschließend ausgestochenen Bodensäule zeigt, dass durch das Saugen nur etwa $10 \%$ der Ameisen, $20 \%$ der Blattläuse, $40 \%$ der Spinnen, $50 \%$ der juvenilen Zikaden und etwa 5-0\% der Asseln, Diplopoda, Chilopoda und Ortheziidae erfasst werden konnten.

In einem Lebensraum mit hoher Vielfalt an Kleinstrukturen sind Saugfänge nicht zur Erfassung von Siedlungsdichten geeignet, da die meisten Tiere nicht aus der Streuschicht herausgesaugt werden können. Die dadurch bewirkte Fehlabschätzung ist zudem hochgradig von der Vegetation abhängig, so dass auch relative Vergleiche stark fehlerbelastet sind. Angesichts des Arbeitsaufwands beim Aussortieren von Proben mit hohem Streuanteil, erscheint der Einsatz des Saugers nur als Ergänzung zu quantitativen Bodenproben sinnvoll, z.B. zur flächenbezogenen Erfassung schnell auffliegender Tiere, die sogar aus der selben Probe wie die spätere Hitzeaustreibung möglich ist. Auch bei einem Zusatzversuch in Rosensträuchern und Baumkronen (vgl. Kap. 7) stellte der Sauger eine gute Ergänzung zum Klopfschirm dar.

\section{Ameisen}

Nun ist die quantitative Bewertung von sozialen Insekten besonders schwierig, bietet aber auch große Vorteile. Der Nachweis einer einzelnen Arbeiterin gibt schon sehr große Sicherheit, dass sich diese Art in unmittelbarer Umgebung erfolgreich etablieren konnte (Seifert 1998); das Problem der Unterscheidung rein zufälliger Einzelfunde von sicheren Nachweisen seltener Arten mit geringen Siedlungsdichten ist nur bei einzeln gefundenen Weibchen relevant. Andererseits ist eine adäquate Abschätzung der Siedlungsdichten bei sozialen Insekten nicht einfach, da die Individuendichte der Arbeiterinnen mit der Entfernung zum Nest exponentiell abnimmt und das zudem noch sehr unregelmäßig, da es bevorzugte Fouragierorte, z.B. Blattlauskolonien, und Wege dorthin, z.B. Ameisenstraßen, gibt. Selbst eine Erfassung der Gesamt-Nestdichte gibt wenig Information über die für andere Tiere und das Ökosystem relevante Biomasse und Aktivität der jeweiligen Ameisenart, da ja die Nester sehr unterschiedlich groß sein können.

Bodenproben bieten den Vorteil, dass sie rein zufällig aus der GesamtPopulation eine Stichprobe ziehen und von daher im Prinzip statistisch auswertbar sind (Köhler et al. 2002). Die starke Klumpung und Schiefe in der Verteilung sollte durch Transformation der Daten zumindest soweit reduzierbar sein, dass statistische Analysen mit gewisser Vorsicht auch zu realistischen Ergebnissen führen (Wilkensen et al. 1996). In der Praxis hat sich gezeigt, dass die Ameisen im Untersuchungsgebiet sehr gut mit Bodenproben der benutzten Größe zu erfassen sind 
und sich die Verteilungen in den Stichproben durch Logarithmieren trotz einiger zufälliger Probenahmen in Nestbereichen überraschend gut in normalverteilte Datensätze mit homogenen Varianzen transformieren lassen (Abb. 9).

Von allen Arten bis auf jene der Gattungen Polyergus und Formica wurden in den Bodenproben regelmäßig Arbeiterinnen gefunden. Unter insgesamt 268 Bodenproben von den Wiesen 1 und 2 gab es nur acht auf Wiese 2, in denen nicht wenigstens eine Arbeiterin gefunden wurde; sieben davon waren aus künstlich beschatteten Parzellen.

Parallel zu dieser Untersuchung wurde in Lebensräumen mit geringer Ameisenpopulationsdichte im Solling eine groß angelegte Waldökosystem-Studie zum Vergleich von Reinbeständen von Rotbuche und Fichte mit Mischbeständen dieser beiden Baumarten durchgeführt (Rothländer et al. 2001, 2003, Sührig et al. 2001). In 90 Kempsonproben aus über 120-jährigen, schon aufgelichteten, Buchen-, Fichten- und Mischbeständen wurden nur vereinzelte, in 90 weiteren Kempsonproben aus 80- bis 120-jährigen Buchen-, Fichten- und Mischbeständen wurden überhaupt keine Arbeiterinnen gefunden. Dennoch gab es auf zwei dieser untersuchten Flächen mit besonders aufgelockerter Kronenschicht und diverser Krautschicht relativ hohe Ameisen-Aktivitätsdichten, wie Bodenfallenfänge zur gleichen Zeit bestätigten: In einem Fichtenbestand im Forstamt Dassel und einem Mischbestand im Forstamt Fürstenberg habe ich dabei überwiegend die Art Myrmica ruginodis Nylander 1846 und regelmäßig, aber vereinzelt die Arten Lasius platythorax Seifert 1991 und Camponotus herculeanus (Linnaeus 1758) feststellen können. Offensichtlich besteht auch hier eine deutliche Abhängigkeit von der Vielfalt an Mikrostrukturen und der Sonneneinstrahlung, die zum Boden gelangt, denn beide Bestände waren schon stark aufgelichtet und hatten eine reich entwickelte Krautschicht (Pfister et al. 2001).

Ohne direkte Nestsuche hätte ich aber z. B. die Amazonenameise Polyergus nicht finden können, denn Arten mit so geringer Nestdichte und schnellen, sich stark optisch orientierenden Arbeiterinnen werden auch nur mit sehr geringer Wahrscheinlichkeit mit einer Bodenprobe erfasst.

Die Beobachtungen an den Köderschalen waren zwar nur bedingt zur Nestsuche geeignet, da auch Krümel forttragende Arbeiterinnen im dichten Grasfilz nicht leicht zu verfolgen waren. Doch die aktuelle, relative Aktivität der einzelnen Arten an der Oberfläche konnte recht gut abgeschätzt werden. Dabei fiel auf, dass es auch thermophilen Ameisen zu heiß werden kann: An sonnigen Sommernachmittagen waren nur noch im Schatten der einzelnen Sträucher Ameisenaktivitäten zu beobachten. Auch für thermophile Arten hat eine reichhaltige VegetationsStruktur, die auch gelegentlich Schatten schafft, offensichtlich Vorteile. 


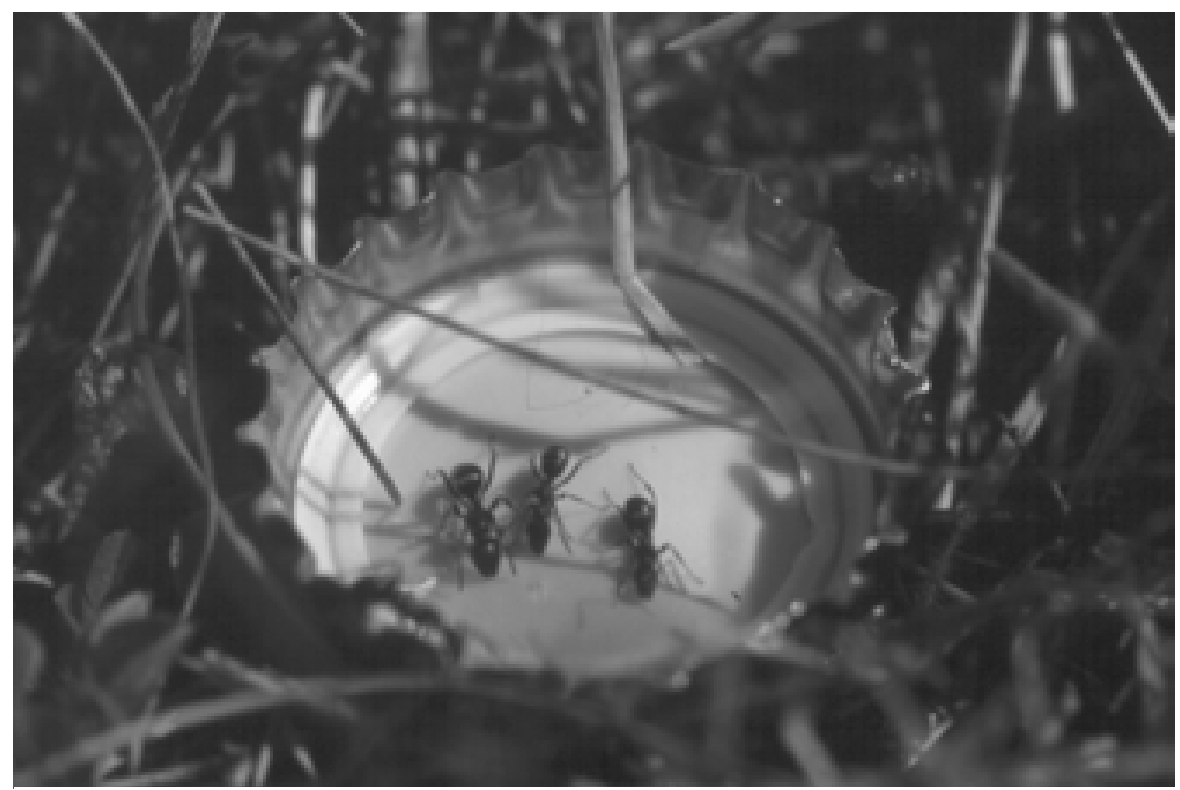

Abb. 20: Lasius alienus an Köderschale mit Honigwasser (Photo: Dirk Sanders)

Formica fusca und cunicularia konnte ich regelmäßig an den Köderschalen beobachten; dabei fiel das zurückhaltende Verhalten der Ameisen auf: sobald sich andere Arten der Nahrungsquelle näherten, zog sich die meist viel größere Formica wieder zurück. Gelegentlich bleiben die Tiere in der Nähe und warten einen günstigen Moment ab, um blitzschnell anderen Arten ihre Beute zu stehlen (Seifert 1996), doch ist das nur eine sehr lockere, gelegentliche Kleptobiose. Die stärker kleptobiotisch lebende Ameise Solenopsis fugax konnte ich nur in Bodenproben finden, ebenso wie Stenamma debile. Die ebenfalls - aus der menschlichen „Vogel"perspektive gesehen - sehr versteckt lebenden Arten Myrmecina graminicola, Ponera coarctata und Tapinoma erraticum wurden auch vereinzelt mit dem Sauger oder in Bodenfallen gefangen.

Prinzipiell fiel mir bei den Verhaltensbeobachtungen auf, dass die meisten Arten sich eher „aus dem Weg gingen“, also deutliches Konkurrenzvermeidungsverhalten zeigten. Auch die Verteidigung eines bestimmten Territoriums scheint einem ökonomischen Kosten-Nutzen-Prinzip zu folgen (Hölldobler \& Lumsden 1980). Holway et al. (1998) stellten an friedlichen Kolonienpaaren der Argentinischen Ameise Linepithema humile eine höhere Produktivität als an aggressiveren Paaren fest und deuten die Reduktion intraspezifischer Aggression als direkten Grund für den großen invasiven Erfolg dieser Ameisenart.

Die eigenen Beobachtungen der Artenzusammensetzung und -verteilung stimmen sehr gut mit den Beschreibungen von Seifert (1986, 1996) und Anderen (Assing 1986, Bauschmann 1988, Rögener \& Pfau 1994, Joger 1995, Sonnenburg 1996, Dauber \& Wolters 2000) zur Habitatwahl der Ameisenarten überein. Bis auf die Waldarten Leptothorax nylanderi und Stenamma debile und die euryöke Art Lasius 
flavus sind alle auf Wiese 1 gefundenen Arten als thermophil bekannt. Bei der Feindifferenzierung z.B. der Leptothorax-Arten sind die Schwerpunkte der Verteilung der einzelnen Arten in der Ordination (Abb. 19) auch sehr gut mit dem umfangreichen publiziertem Vorwissen in Einklang zu bringen. Auch das Fehlen weitverbreiteter Arten wie Myrmica scabrinodis passt dank der umfangreichen systematischen, morphologischen und ökologischen Arbeiten von Bernhard Seifert in das Gesamtbild: Auf trockenen Standorten ist die Wahrscheinlichkeit, Myrmica scabrinodis zu finden, nach seinen Erfahrungen nur sehr gering. Viele anderslautende Angaben führt er auf Verwechselungen mit der sehr ähnlichen Art Myrmica sabuleti zurück (Seifert 1986).

\subsubsection{Räumliche Beziehungen zur übrigen Tierwelt und zur Umwelt}

Enge Assoziationen zwischen Arten und Zentroiden der Habitattypen in der Hauptkomponentenanalyse, wie z.B. zwischen Regenwürmern und Trockenrasen (Abb. 11), müssen nicht immer bedeuten, dass das Taxon gerade diesen Lebensraumtyp bevorzugt. Die zweidimensionale Darstellung ist diejenige mit dem höchsten Gesamterklärungswert, es kann sehr gut möglich sein, dass durch eine weitere, nicht dargestellte Achse zwei assoziiert erscheinende Punkte wieder getrennt werden. Die Dichte der Regenwürmer war im Hain im Mittel mit 406 Ind. $/ \mathrm{m}^{2}$ deutlich über derjenigen auf dem Trockenrasen mit 233 Ind. $/ \mathrm{m}^{2}$. Dennoch hatten die Würmer auf dem Trockenrasen unerwartet eine deutlich höhere Dichte als im Wald (126 Ind. $/ \mathrm{m}^{2}$ ). Die hohen Mittelwerte der Individuendichte liegen an einem „hot spot“ am Rasterpunkt A7 im Wacholder-OrchideenAbschnitt des Halbtrockenrasens und an den hohen Dichten in den Übergangszonen zur Brachwiese. Aufgrund der nicht immer einfachen Zuordnung der Probenahmestellen zu den Lebensraumtypen ist diese Kategorie auch nur als passive Variable in die Analyse eingegangen.

Für räumliche statistische Verfahren ist der „Seitenarm“ am Raster sehr ungeeignet, bei der deskriptiven graphischen Analyse kann er jedoch wertvolle Zusatzinformationen enthalten. Beispielsweise lässt sich an der Verteilung vieler Variablen immer wieder eine Grenzlinie im Übergangsbereich der beiden Wiesentypen, etwa auf Höhe der „Abzweigung“ dieses Transekts beobachten, die sich auch auf der Nachbarfläche 1a wiederfindet (Abb. 15-18). Die Thysanoptera wurden fast nur in dieser „Grenzzone“ außerhalb des Waldes gefunden. Solche „Sprünge“ in räumlichen Verteilungen können historische Ursachen haben: etwa genau auf dieser Grenzzone verläuft eine Grundstücksunterteilung (vgl. Abb. 2); vermutlich wurden die beiden Teile noch vor der zurückliegenden Beweidung durch Rinder, die auf der ganzen Fläche stattfand (Müller, mündl. Mitteilung), unterschiedlich genutzt. Bei der Formica pratensis-Kolonie auf Fläche 1a ist etwa auf dieser Linie eine Terrassenstufe, etwas weiter nördlich sogar ein Mauer-, bzw. Steinwallrest erkennbar, der vermutlich noch aus der Weinbauzeit stammt. Der Boden hat ein „langes Gedächtnis“, manche Humusformen ändern sich über Jahrzehnte nur sehr wenig. Selbst nach 39 Jahren Maisanbau wurden auf einem Versuchsfeld bei Halle nur etwa 10-20 \% des schon vorher festgelegten Kohlen- 
stoffs im Boden ausgetauscht, wie an den Delta ${ }^{13} \mathrm{C}$-Werten erkennbar ist (Ludwig et al 2003). So bleibt bei dem deutlich mit den Tieren der Brachwiese korrelierendem $\mathrm{pH}$-Wert die Frage offen, ob dieser schon lange im unteren Bereich höher war, oder ob nicht erst die unterschiedliche aktuelle Entwicklung der Vegetation zu einer langsamen Verschiebung geführt hat. Im Bereich schon länger verbuschter Bereiche, wie dem Hain ist der pH-Wert jedenfalls schon erkennbar niedriger. Jüngere Verbuschung hat bisher weder den $\mathrm{pH}$-Wert ändern können noch die Siedlungsdichten der Ameisen. Dies könnte an einer langsamen Wirkung veränderter Umweltbedingungen auf die Ameisen liegen - im Sinne eines „Langzeitgedächtnisses" für den Standort (Steiner \& Schlick-Steiner 2002) - oder ein Effekt des aktiven Nestbaus hügelbauender Arten wie Lasius flavus sein, der einerseits diese gewisse Unabhängigkeit von kurzfristigen Veränderungen der Vegetation überhaupt erst ermöglicht, aber andererseits auch direkt wieder modulierend den Boden und das Pflanzenwachstum beeinflusst (King 1977b, 1981).

Die Signifikanzen der Monte-Carlo-Tests habe ich noch mal für das Kernraster, also ohne den Extra-Transekt, unter Berücksichtigung einer räumlichen Verbundenheit überprüft (Tab. 8). Die Ergebnisse legen nahe, dass die für die dargestellten Ordinationen gefundenen signifikanten Effekte auch unter Berücksichtigung der räumlichen Struktur realistisch sind.

Tabelle 8: Eigenwerte und Varianzaufklärungen der Arten-Umwelt-Relation (VA) der ersten vier Achsen je einer Hauptkomponentenanalyse (PCA) und einer Redundanzanalyse (RDA) mit den Individuenzablen der Tiergruppen aus den Bodenproben der Rasteruntersuchung für ein 7x9und ein 6x10-Kernraster $\left(\mathrm{RD} A_{63}, \mathrm{RD} A_{60}\right)$ und das ganze Raster ( $\mathrm{RD} A_{83}$, obne Seitenarm) mit 20 (RDA63, $\left.\mathrm{RD} A_{60}\right)$ bzw. 2 ( $\mathrm{RD} A_{83}$, den X,Y-Koordinaten) Umweltvariablen (UV). Der erklärte Anteil (VA) der Artenvarianz ist bei linearen Verfahren (PCA, RDA) gleich dem Eigenwert der jeweiligen Achse. F-Werte und Signifikanzniveaus je eines Monte-Carlo-Tests mit 999 restriktiven Permutationen (J) für rechteckige Raster $\left(\mathrm{RD} A_{63}, \mathrm{RD} A_{60}\right.$; toroidal shifts; (*): $p<0,1$; *: $p<0.05$; **: $p<0,01)$ bzw. mit nicht-restriktiven Permutationen ( $\mathrm{RD} A_{83}$; ***: $\left.p=0,001\right)$.

\begin{tabular}{|c|c|c|c|c|c|c|c|c|}
\hline & \multicolumn{2}{|c|}{ 1. Achse } & \multicolumn{2}{|c|}{ 2. Achse } & \multicolumn{2}{|c|}{ 3. Achse } & \multicolumn{2}{|c|}{ 4. Achse } \\
\hline & Eig & $\begin{array}{l}\mathrm{VA} \\
(\%)\end{array}$ & Eig & $\begin{array}{l}\mathrm{VA} \\
(\%)\end{array}$ & Eig & $\begin{array}{l}\mathrm{VA} \\
(\%)\end{array}$ & Eig & $\begin{array}{l}\text { VA } \\
(\%)\end{array}$ \\
\hline \multicolumn{9}{|l|}{$\mathrm{B} 1-\mathrm{H} 9(7 \times 9)$} \\
\hline $\mathrm{PCA}_{63}$ & 0,316 & & 0,122 & & 0,082 & & 0,065 & \\
\hline $\mathrm{RDA}_{63,20}$ & 0,226 & 47,8 & 0,057 & 12,1 & 0,039 & 8,4 & 0,031 & 6,5 \\
\hline Mte Carlo & \multicolumn{2}{|c|}{$12,25 *$} & \multicolumn{6}{|c|}{$1,88^{* *}$} \\
\hline \multicolumn{9}{|l|}{$\mathrm{B} 1-\mathrm{G} 10(6 \times 10)$} \\
\hline $\mathrm{PCA}_{60}$ & 0,3 & & 0,131 & & 0,098 & & 0,065 & \\
\hline $\mathrm{RDA}_{60,20}$ & 0,206 & 42,1 & 0,075 & 15,4 & 0,066 & 13,5 & 0,026 & 5,4 \\
\hline Mte Carlos & \multicolumn{2}{|c|}{10,13 (*) } & \multicolumn{6}{|c|}{$1,87^{*}$} \\
\hline \multicolumn{9}{|l|}{ A1 - H9 } \\
\hline $\mathrm{RDA}_{83, \mathrm{XY}}$ & 0,105 & 82,5 & 0,022 & 17,5 & 0,22 & 0 & 0,15 & 0 \\
\hline Mte Carlo & \multicolumn{2}{|c|}{$9,38 * * *$} & \multicolumn{6}{|c|}{$5,83 * * *$} \\
\hline
\end{tabular}


Auf weitergehende räumliche Analysen wird hier verzichtet, da die zusammenhängenden Bereiche gleicher Habitattypen zu klein für separate Auswertungen sind und eine Auswertung über die ganze Fläche eine sehr hohe Autokorrelation ergeben würde, die allein durch die Flecken gleicher Habitate zustande käme.

Beschattung hat einen eher negativen Effekt auf die Ameisen: im Wald und in dichteren Gebüschen kommen weniger Arten mit geringeren Siedlungsdichten vor. Die konkrete Ausprägung des Habitats kann die Ameisen stark beeinflussen. Die Ameisen zeigen tendenzielle Reaktionen auf die mikroklimatischen Faktoren Bodenwassergehalt (negativ), Lufttemperatur und relative Luftfeuchte (beide positiv), die Korrelationen sind aber nicht signifikant.

Die einzelnen Arten reagieren ja auch durchaus differenziert auf unterschiedliche biotische und abiotische Bedingungen. Entsprechend werden die Gradienten auf Artebene meist deutlicher als auf höherem taxonomischem Niveau, wie auch ein Vergleich der Eigenwerte der Ordinationen zeigt (Tab. 5 \& 7). Die Spinnenarten zeigen auf dem Gradientenausschnitt innerhalb der Brachwiese keine deutliche Gruppierung, im Gegensatz zu den Zikadenarten. Auf einer stärker funktionellen Ebene werden die Unterschiede aber auch bei den Spinnen deutlicher: Netzspinnen und vagante Jäger reagieren sehr distinkt auf die Struktur- und Mikroklimaunterschiede. Innerhalb dieses kleineren Gradienten tragen die erfassten Umweltvariablen auch für die Ameisenarten wenig zum Erklärungswert bei (Tab. 7 a); auf dem größeren Gradienten mit deutlicheren Habitatunterschieden korrelieren die abiotischen Umweltvariablen hingegen gut mit der Verteilung der Arten (Tab. 7 b). Die deutlichen Unterschiede in den Artengemeinschaften entlang der ganzen Reihe von Habitattypen beeinflussen dann trotz des gewissen Kompensationseffekts auch wieder die Dichten und Verteilungen der ganzen Tiergruppe. Insgesamt spiegelt die Ordination der Tiergruppen damit oft auch die Grundzüge der Artenverteilung wider, sofern die Gruppe insgesamt nicht indifferent reagiert und damit in das Zentrum ordiniert wird.

Das Habitat hat auf jeden Fall einen deutlichen Einfluss sowohl auf die Ameisen- als auch auf die gesamte Tiergemeinschaft, der aber in seiner Richtung stark von der konkreten Ausprägung abhängt; für Pflanzen und Ameisen bedeutet das, einfach formuliert, Bäume wirken, sobald sie älter werden, negativ, Gras und Moos positiv auf die Ameisen.

Aus Korrelationen können aber nicht ohne Weiteres Rückschlüsse auf Kausalitäten geschlossen werden, dazu bedarf es experimenteller Nachweise.

\subsubsection{Interaktionen mit Saprophagen, Phytophagen und Zoophagen?}

Bei separaten, einfachen Korrelationsanalysen der jeweils logarithmierten Dichten der Ameisen mit denen anderer Tiergruppen konnten sechs signifikante positive Beziehungen festgestellt werden.

Die Ameisen korrelieren mit den Spinnen sehr schwach positiv $\left(r^{2}=0,05\right.$; $\mathrm{p}=0,04)$ und mit den Erdläufern schwach positiv $\left(\mathrm{r}^{2}=0,12 ; \mathrm{p}<0,001\right)$; bei einer Korrektur des Signifikanzniveaus nach Bonferroni-Holm ist die Korrelation mit den Spinnen als einzige der sechs nicht mehr signifikant. 
Die vier übrigen positiven Beziehungen der Ameisen gab es zu den überwiegend phytophagen, pflanzensaftsaugenden Taxa der Hemiptera (**: $p<0,01$; $* * *: \mathrm{p}<0,001)$ :

- Wanzen

- Zikaden

- Blattläuse

- Röhrenschildläuse

$$
\begin{aligned}
& r^{2}=0,14 * * * \quad \text { (sehr viele Tingidae) } \\
& r^{2}=0,27 * * * \\
& r^{2}=0,27 * * * \\
& r^{2}=0,10^{* *}
\end{aligned}
$$

Aus den rein deskriptiven Daten kann zusammenfassend geschlossen werden, dass in einem Mosaik verschiedener Habitate die Ameisen insgesamt positiv mit Pflanzensaftsaugern korrelieren, mit einigen Zoophagen (Araneida, Geophilidae) schwach positiv und mit der saprophagen Makrofauna tendenziell auch eher positiv (Isopoda, Lumbricidae), wenn auch nicht signifikant. Die eher negativen Beziehungen zu Elateridae, Lithobiidae und Julidae sind nur in Tendenzen erkennbar.

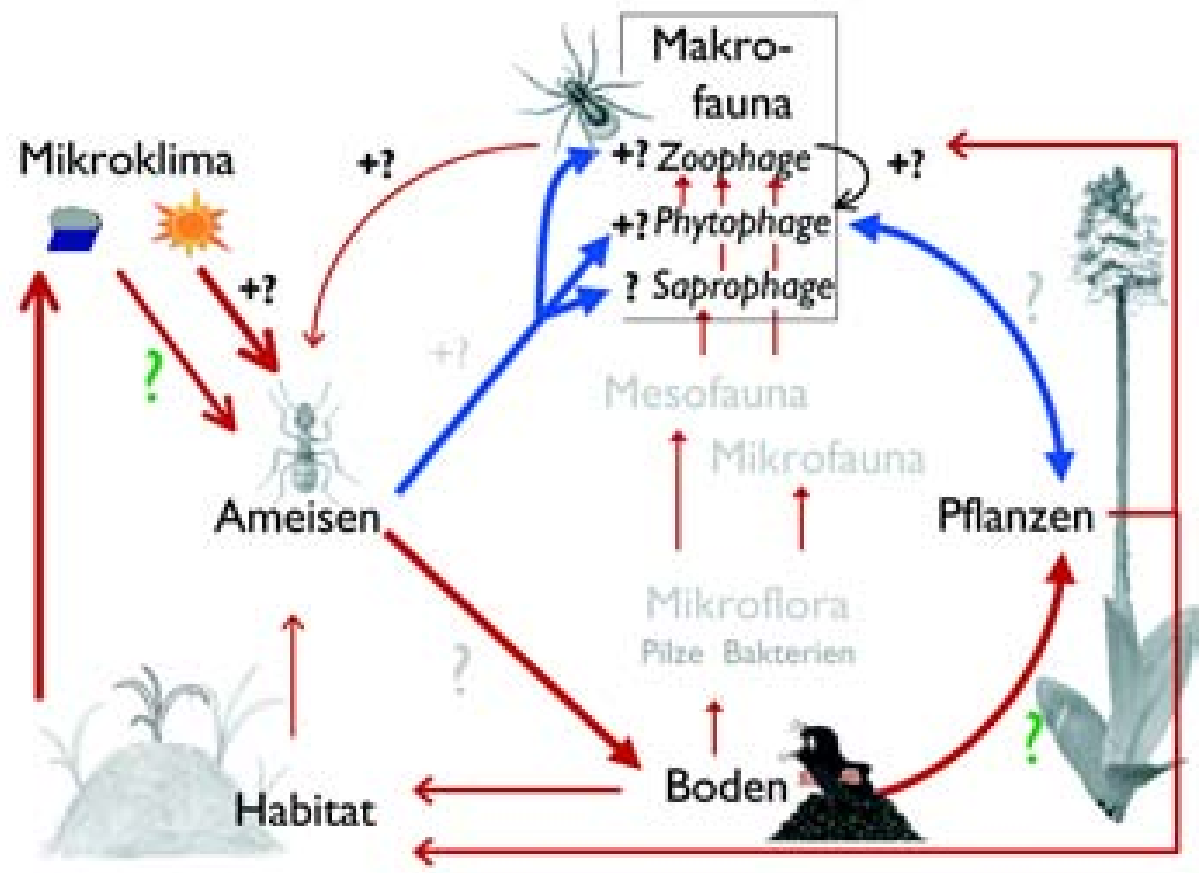

Abb. 21: Mögliche Wechselwirkungen zwischen Mikroklima, Ameisen und der Makrofauna aufgrund festgestellter Korrelationen in der Rasteruntersuchung 


\section{Einfluss des Mikroklimas: Auswirkungen expe- rimenteller Veränderungen auf die Bodenmakro- fauna einer trockenen Glatthaferwiese}

\subsection{Einleitung}

Die Klimafaktoren Sonneneinstrahlung und Niederschlag sind die wesentlichen Quellen der Ressourcen Licht, Wärme und Wasser, von deren Verfügbarkeit direkt oder indirekt alle Kompartimente und Stoffflüsse von Ökosystemen abhängen. Nur wenige Punkte in der Biosphäre haben aktuell das jeweils richtige Mikroklima für ihre Bewohner; Ameisen beispielsweise sind in ihrem Vorkommen und ihrer Aktivität auf bestimmte klimatische Bedingungen angewiesen und beeinflussen ihrerseits viele andere Tiergruppen, biologische Vorgänge und Bodeneigenschaften. Wie alle Insekten suchen auch fouragierende Ameisen unter den verfügbaren die für sie geeignetsten mikroklimatischen Bedingungen auf. Als soziale Insekten schaffen sie sich mit ihrem Nest allerdings auch einen Ort, der sie vor ungünstigen Bedingungen schützt und ein eigenes, möglichst günstiges Mikroklima bewahrt (Brian 1983). Andererseits wird ein Nest mit zunehmender Komplexität immobiler und muss dadurch über längere Zeiträume den äußeren Umweltbedingungen, die gerade an der Bodenoberfläche stark schwanken, trotzen können. Die Sonneneinstrahlung am Neststandort ist ein entscheidender Faktor in der Biologie vieler Ameisen, beispielsweise für die Verbreitung von Myrmica-Arten im gemäßigten Europa (Elmes \& Wardlaw 1982); selbst die eurytope Art Myrmica rubra benötigt für die Aufzucht von Geschlechtstieren eine Temperatur von knapp $20{ }^{\circ} \mathrm{C}$ 
(Brian 1973). Die Wahl des richtigen Nistplatzes, z.B. unter einem flachen Stein, kann für das Überleben und den Erfolg der Kolonie entscheidend sein.

Volkreiche Kolonien einiger Arten emanzipieren sich stärker durch die Anlage eines Nesthügels, der in besonderer Weise gleichbleibend gute mikroklimatische Bedingungen bietet (z.B. Kneitz 1970, Dlussky 1981). Doch auch die hügelbauende Wiesenameise Lasius flavus gründet ihre Kolonien vorwiegend an gut drainierten, südexponierten Standorten (Waloff \& Blackith 1962) und wird in ihrer Verteilung durch Beschattung limitiert (Pontin 1963). Dlussky (1981) berichtet, dass die Lasius flavus - Kolonie ihre Larven- und Puppenkammern bevorzugt auf der oberen Südseite ihres Hügels anlegt und ihn dort auch verstärkt ausbaut; auf der ungenutzten Nordseite siedeln öfters Myrmica-Arten. Durch aufwachsende hohe Vegetation beschattete und verlassene Nesthügel werden oft ganz von Myrmica spec. oder Lasius niger besiedelt (Woodell \& King 1991).

Andere für die Steuerung ökologischer Umsatzprozesse wichtige Tiere, wie die saprophagen Taxa Lumbricidae, Diplopoda und Isopoda und die pantophagen Gastropoda, bevorzugen im Unterschied zu den Ameisen eher feuchte und dunkle Orte und damit meist ganz andere ökoklimatische Bedingungen. Auch auf dem globalen Gradienten von temperierten zu tropischen Regionen, einem der offensichtlichsten und ältesten ökologischen Trends (z.B. Forster 1778), der in erster Linie durch Unterschiede von Sonneneinstrahlung und Niederschlägen geprägt wird (von Humboldt 1808, Hawkins 2004), lässt sich eine Verschiebung der Bedeutung der Boden-Ökosystemingenieure Regenwürmer und Ameisen gegeneinander erkennen: In temperierten Wäldern, Grasländern und Agrarsystemen nehmen sehr oft Lumbricidae die zentrale ,grabende“ Rolle der Bodengestalter ein, in den meisten tropischen Lebensräumen sind es eher die sozialen Insekten Ameisen und Termiten.

Globaler Klimawechsel ebenso wie lokale Suksessionsprozesse können die Diversität und Zusammensetzung natürlicher Gemeinschaften ändern, was insbesondere über den Boden direkt auf die „Dienstleistungsfähigkeit“ eines Ökosystems rückwirken kann. Trotz der enormen Bedeutung des Mikroklimas für die Verteilung von Ameisen und anderen Bodentieren wurden bisher vergleichsweise wenig Freilandexperimente zur Auswirkung von Klimaveränderungen durchgeführt.

Um die Abhängigkeit der Bodentiergemeinschaft vom Mikroklima und damit auch mögliche Folgen durch Klima- bzw. Biotopveränderungen unter möglichst natürlichen Bedingungen zu untersuchen, wurden auf einer wärmebegünstigten Glatthaferwiese die beiden wichtigsten klimatischen Faktoren, Sonneneinstrahlung und Niederschläge, also Energie- und Wassereintrag, experimentell durch Beschattung, Austrocknung bzw. zusätzliche Beregnung verändert.

Durch eine breite Analyse der Tiergemeinschaft und der Bodenbedingungen, sowohl relativ kurz nach Beginn der Manipulation als auch nach längerer Einwirkung, können Rückschlüsse auf die relative Bedeutung direkter Wirkungen des Mikroklimas und indirekter Effekte z.B. über Veränderungen der Habitatstruktur oder des Nahrungsangebots, gezogen werden. 
Für Ameisen zeigte sich schon zu Beginn neben der erwarteten deutlich positiven Wirkung der Sonneneinstrahlung eine ebenfalls positive Tendenz zusätzlicher Beregnung. Nach der Diskussion der ersten Ergebnisse auf einer internationalen Tagung in Budweis führte das zu neuen Detailhypothesen zur besonderen Auswirkung der Mikroklimaveränderungen innerhalb der Nesthügel von Ameisen: Bei zusätzlicher Beregnung stiegen tendenziell sowohl die Wassergehalte, die mikrobielle Biomasse als auch die Dichte der Ameisen außerhalb des Nesthügels an. Diese Zusammenhänge könnten ihre Ursache in ausgeglicheneren Temperaturverläufen im Nest haben, entweder durch die hohe Wärmekapazität des Wassers oder sogar durch die Metabolismuswärme der Mikroorganismen (Frouz 2000); die höhere mikrobielle Aktivität unter feuchteren Bedingungen könnte die nächtliche Temperaturabsenkung etwas abmildern. So könnten Ameisen-Kolonien und Mikroflora voneinander profitieren. In einem kleineren Zusatzversuch mit gleichzeitiger Messung der mikrobiellen Biomasse sowie der Temperatur und relativen Luftfeuchte im Boden wurde diese Hypothese im Sommer 2002 überprüft.

Die in beiden Versuchen zusätzlich ermöglichten bodenbiologischen Vergleiche zwischen Boden aus den Nesthügeln der Ameisen und von der umgebenden Wiese werden zusammen mit den weiteren, umfassenderen Bodenuntersuchungen im Kapitel 8 behandelt.

\subsection{Methodik}

Im Werratal bei Witzenhausen-Freudenthal wurden auf einer brachliegenden, südexponierten ehemaligen Rinderweide auf Kalkgestein („Wiese 2“, Kap. 2), auf der Hügelnester der gelben Wiesenameise (Lasius flavus) in hoher Dichte vorkommen, die Faktoren „Sonne“ und „Regen“ experimentell verändert: Die Sonneneinstrahlung wurde mit regendurchlässigen Schattendächern aus PE-Geflecht (Abb. 22) vermindert, die Niederschlagsmenge mit lichtdurchlässigen Regendächern aus Klarsichtfolie und später Plexiglas (Abb. 23) minimiert bzw. durch zusätzliches Beregnen mit demineralisiertem Wasser $\left(101 \times \mathrm{m}^{-2} \times 14 \mathrm{~d}^{-1}\right)$ auf etwa das 1,5fache der natürlichen Menge gesteigert.

Die sechs Varianten eines orthogonalen Versuchsdesigns mit dem zweistufigen Faktor „Sonne“ und dem dreistufigen Faktor „Regen“ wurden je fünf mal wiederholt. Dazu wurden im April 1998 auf der Versuchsfläche 30 Hügelnester von Lasius flavus gesucht und zusammen mit jeweils einem hangabwärts gelegenen Quadratmeter Wiese als Versuchsparzelle markiert bzw. durch Aufstellen der Dächer eingerichtet. Die jeweils gleiche Ausrichtung der Vergleichswiesenfläche zum Nesthügel verminderte die unkontrollierte Beeinflussung sowohl durch den Hügelschatten als auch durch Hangabflusswasser.

Die Varianten wurden jeweils innerhalb relativ homogener Bereiche der Gesamtfläche zufällig den einzelnen Parzellen zugeordnet (Blockanlage mit randomisierter Anordnung), die räumliche Anordnung der Parzellen innerhalb dieser Blöcke war durch die Lage vergleichbarer Nesthügel vorgegeben (Abb. 24). 


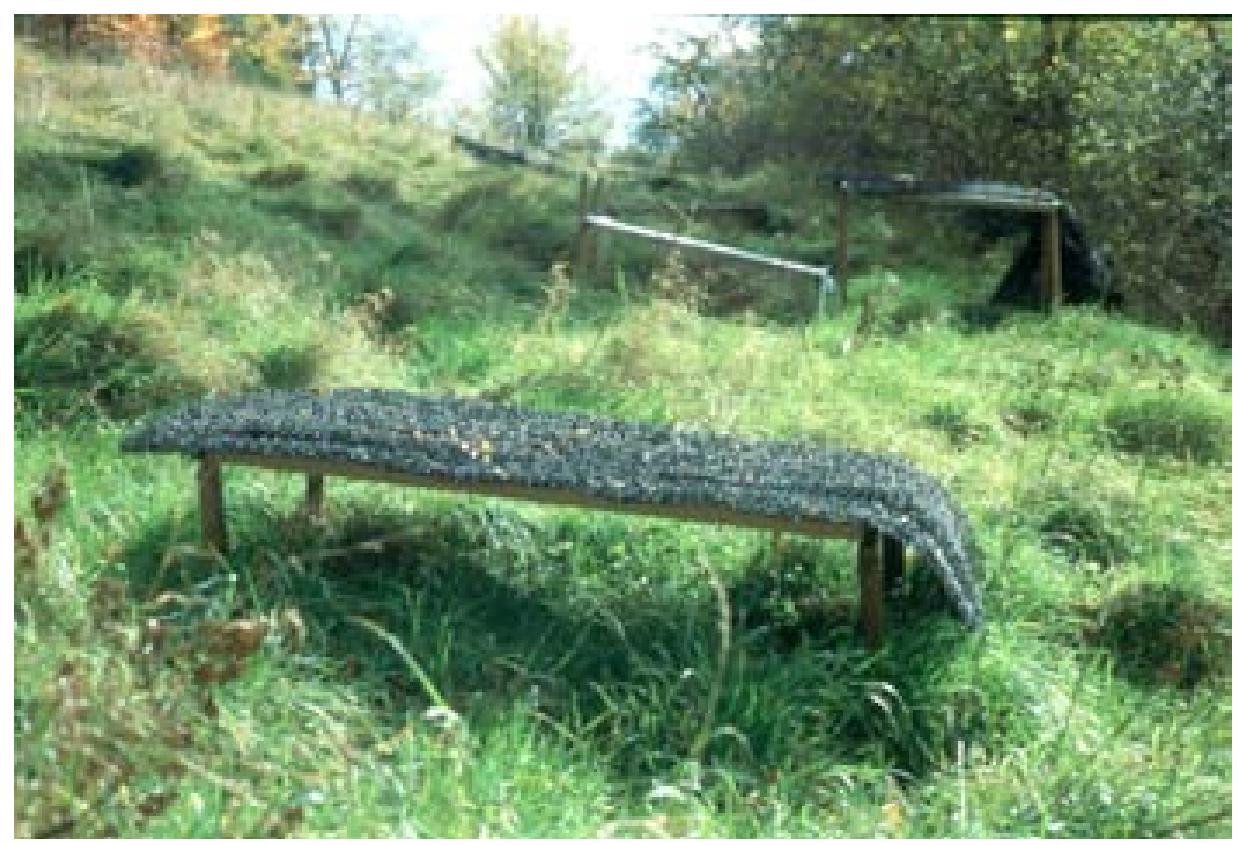

Abb. 22: Reduktion der Sonneneinstrablung mit einem regendurchlässigen Schattendach.

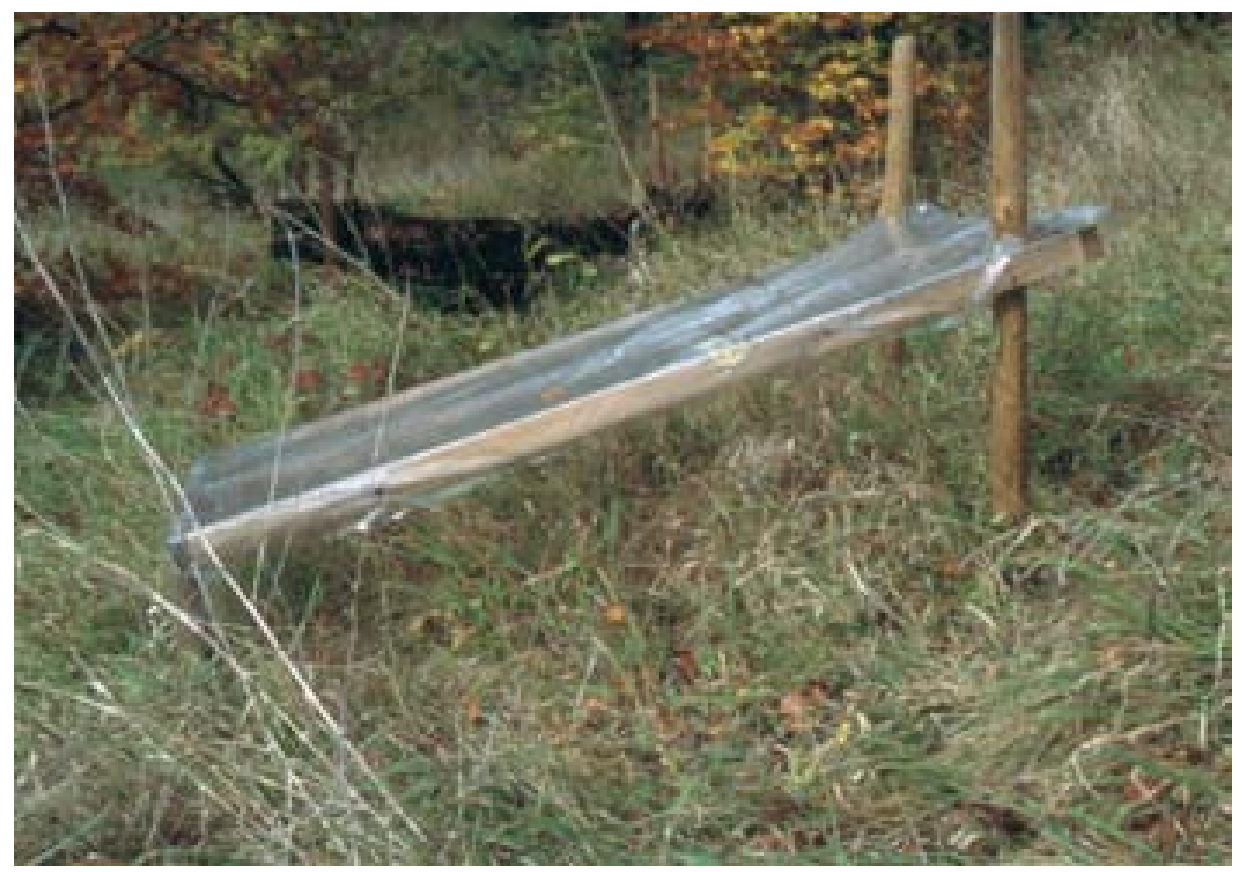

Abb. 23: Reduktion des Niederschlags mit einem lichtdurchlässigen Regendach 
Am 13. August 1998, 3. Mai 1999 und 26. April 2000, also ein Vierteljahr, ein Jahr und zwei Jahre nach Beginn der Manipulation, wurden die Tiergemeinschaft und wichtige Bodenparameter beprobt. Aus einer Bodenprobe vom Wiesenteil jeder Parzelle mit dem großen Bohrer (Kempson-Proben, Kap. 3.1.) wurde die Makrofauna durch Hitzeextraktion ausgetrieben; aus je drei Einstichen mit dem kleinen Bohrer wurden nach Sieben und Homogenisieren die Nematoden ausgetrieben und die mikrobielle Biomasse durch Messung der SIR (Kap. 3.3), der Kohlen- und Stickstoffgehalt gaschromatographisch und der Wassergehalt gravimetrisch (105 ${ }^{\circ} \mathrm{C}, 24 \mathrm{~h}$ ) bestimmt. Zusätzlich wurde an jedem Termin der Ameisen-Nesthügel randlich vorsichtig mit dem McFadyen-Bohrer angestochen, um den Zustand der Lasius flavus Kolonie abzuschätzen bzw. die Besiedlung durch andere Ameisenarten festzustellen.

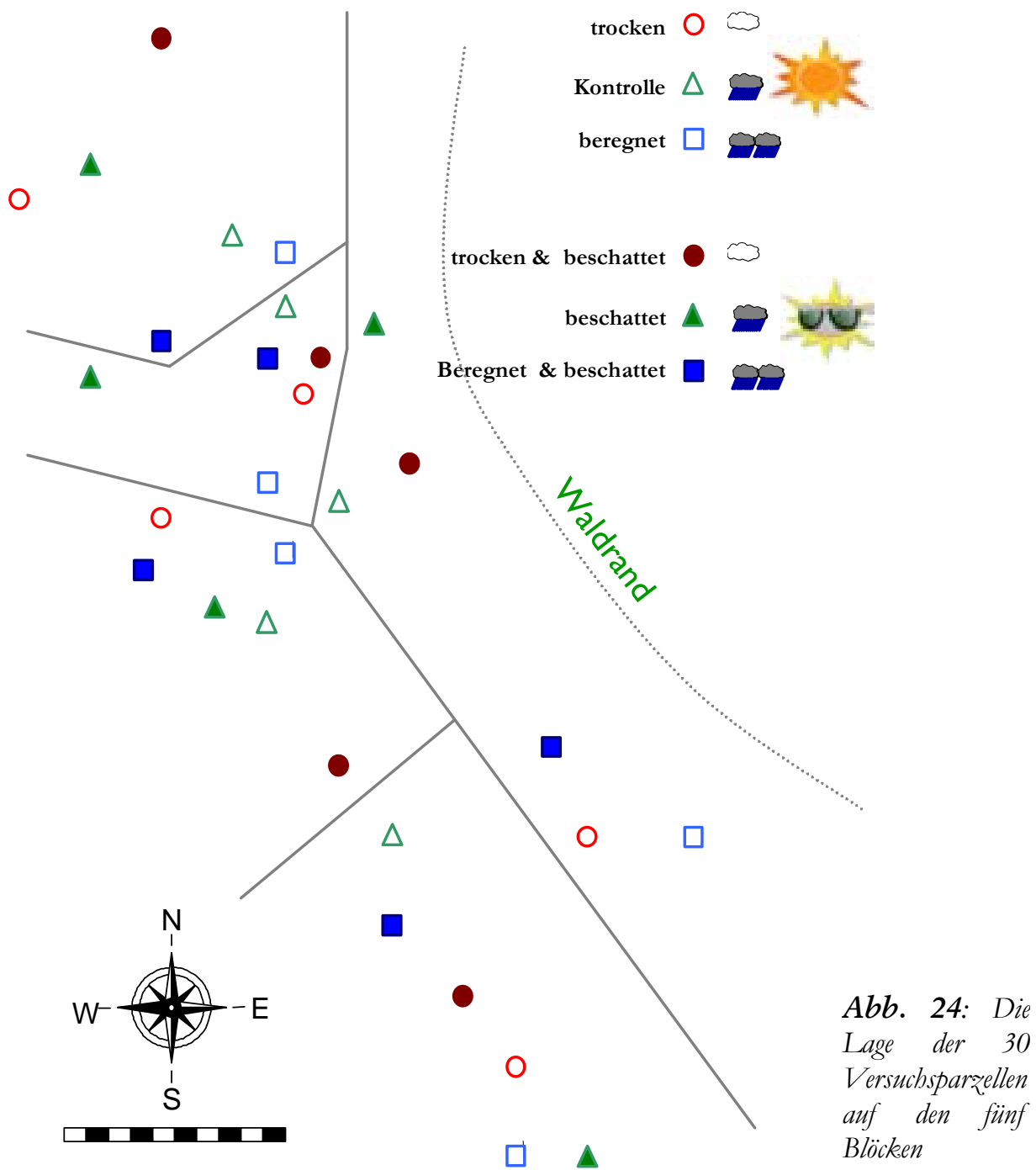




\subsection{Ergebnisse}

\subsubsection{Auswirkungen auf den Boden}

\section{Abiotische Bedingungen}

Die Wassergehalte des Bodens veränderten sich signifikant entsprechend der Behandlung sowohl innerhalb der Ameisennester (Abb. 26 c, Tab. 10) als auch auf den Wiesenparzellen (Abb. 25 a, Tab. 9). Die C- und N-Gehalte unterliegen deutlich räumlichen Gradienten, wobei der Faktor Sonne den C-Gehalt unter Berücksichtigung des Blockeffektes signifikant beeinflusst (Abb. 25, Tab. 9; besonnte Flächen: 4,53 g C/g TG Boden; beschattete Flächen: 4,07 g C/g TG Boden).

In den Ameisennestern wurde die Temperatur durch Beschattung signifikant herabgesetzt (Tab. 10). In den ausgetrockneten, beschatteten Nestern waren aber auch die Schwankungen deutlich geringer. Zusätzliche Beregnung erhöhte in der Sonne die Schwankungsbreite, reduzierte sie aber im Schatten.
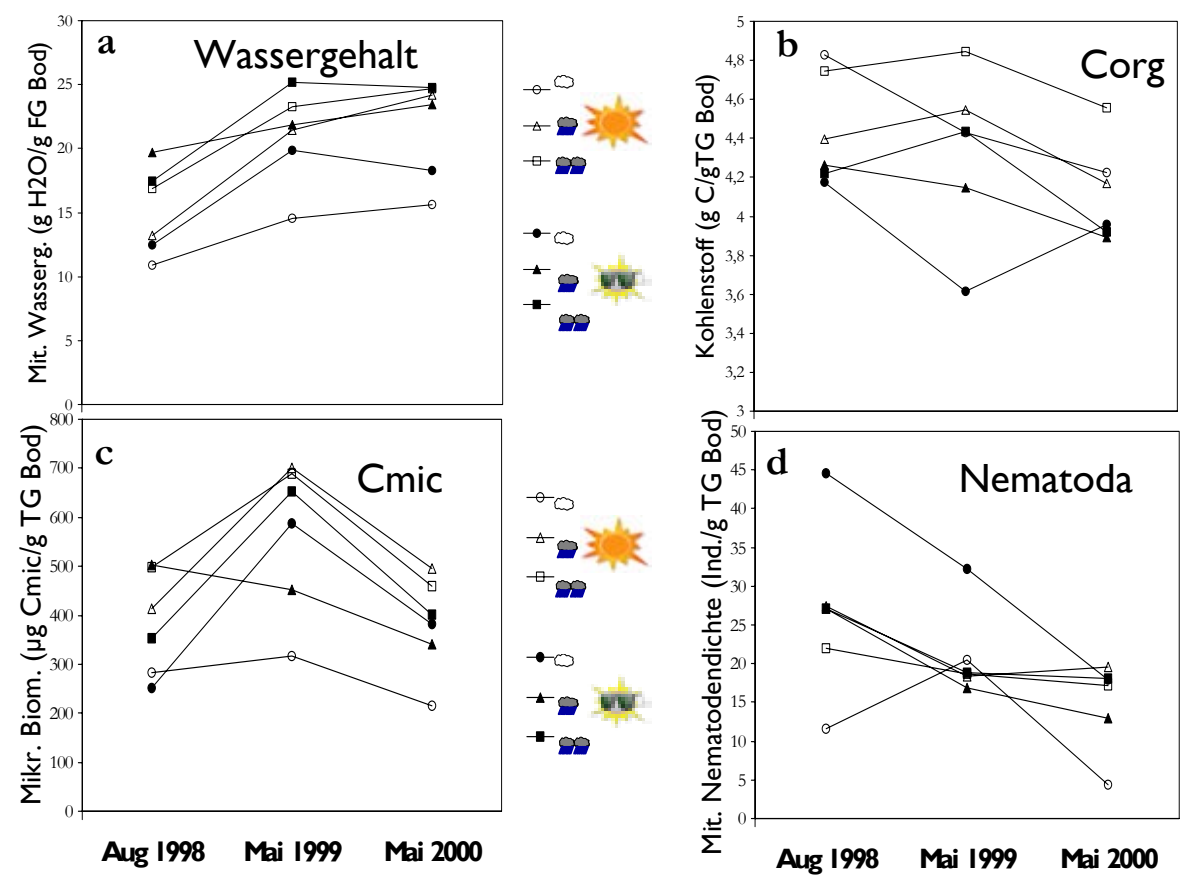

Abb. 25: Mittlerer Wassergehalt (a), Gesamt-Koblenstoffgehalt (b), mikrobielle Biomasse (c) und Nematodendichte (d) in $0-5 \mathrm{~cm}$ Bodentiefe auf den Wiesenparzellen; jeweils eine Mischprobe aus drei Einstichen MacFadyen; Symbole sind in Abb. 24 erklärt: dunkle stehen für Beschattung, je mehr Ecken, um so mehr Niederschlag. 
Tabelle 9: F-Werte und Wabrscheinlichkeiten für $\alpha$-Fehler (p) der Behandlungs-, Block- und Termineffekte und deren Interaktionen aus zweifaktoriellen Varianzanalysen für Messwiederholungen der Bodenanalysen und der Fadenwurmdichten von den Wiesenparzellen nach der Mikroklimamanipulation. FG: Freiheitsgrade (Effekt; Febler); innerbalb der Behandlungsstufen FWerte des multivariaten Pillai trace Tests; $p<0,05:$ F-Werte fett gesetot; $p>0,3$ nicht angegeben; $\log _{\text {: }}$ Werte log-transformiert $\left(\log _{10} X\right)$.

a) repeated measurement

\begin{tabular}{|c|c|c|c|c|c|c|c|c|c|}
\hline & \multirow[b]{2}{*}{$\mathrm{FG}$} & \multicolumn{2}{|c|}{ Wassergehalt } & \multicolumn{2}{|c|}{ Kohlenstofflog } & \multicolumn{2}{|c|}{$\mathrm{Cmic}^{\log }$} & \multicolumn{2}{|c|}{ Nematoda ${ }^{\log }$} \\
\hline & & $\mathrm{F}$ & $\mathrm{p}$ & $\mathrm{F}$ & $\mathrm{p}$ & $\mathrm{F}$ & $\mathrm{p}$ & $\mathrm{F}$ & $\mathrm{p}$ \\
\hline Regen & $2 ; 20$ & 38,30 & $<0,0001$ & 0,68 & & 4,68 & 0,0215 & 0,78 & \\
\hline Sonne & $1 ; 20$ & 6,26 & 0,0212 & 5,84 & 0,0253 & 0,00 & & 9,95 & 0,0050 \\
\hline $\mathrm{R} \times \mathrm{S}$ & 2,20 & 1,31 & 0,2927 & 0,25 & & 2,09 & 0,1494 & 13,02 & 0,0002 \\
\hline Block & $1 ; 20$ & 5,84 & 0,0028 & 2,86 & 0,0503 & 0,77 & & 0,34 & \\
\hline Zeit & $2 ; 19$ & 35,94 & $<0,0001$ & 18,83 & $<0,0001$ & 21,15 & $<0,0001$ & 15,40 & 0,0001 \\
\hline $\mathrm{Z} \times \mathrm{R}$ & $4 ; 40$ & 1,39 & 0,2547 & 1,76 & 0,1554 & 0,47 & & 3,44 & 0,0166 \\
\hline $\mathrm{Z} \times \mathrm{S}$ & $2 ; 19$ & 1,28 & & 0,23 & & 0,22 & & 2,66 & 0,0961 \\
\hline $\mathrm{Z} \times \mathrm{R} \times \mathrm{S}$ & $4 ; 40$ & 1,28 & 0,2925 & 1,55 & 0,2065 & 1,84 & 0,1408 & 1,50 & 0,2209 \\
\hline $\mathrm{Z} \times \mathrm{Bl}$ & $8 ; 40$ & 0,97 & & 1,12 & & 0,85 & & 0,52 & \\
\hline \multicolumn{10}{|c|}{ b) ANOVA August 1998} \\
\hline Modell & $9 ; 20$ & 3,51 & 0,0093 & 2,70 & 0,0308 & 1,18 & & 2,68 & 0,0318 \\
\hline Regen & $2 ; 20$ & 6,36 & 0,0073 & 0,28 & & 2,37 & 0,1189 & 0,38 & \\
\hline Sonne & $1 ; 20$ & 4,60 & 0,0444 & 5,03 & 0,0365 & 0,17 & & 9,12 & 0,0068 \\
\hline $\mathrm{R} \times \mathrm{S}$ & 2,20 & 1,84 & 0,1852 & 0,63 & & 0,52 & & 6,07 & 0,0087 \\
\hline Block & $4 ; 20$ & 2,64 & 0,0642 & 4,36 & 0,0107 & 1,17 & & 0,52 & \\
\hline \multicolumn{10}{|c|}{ c) ANOVA Mai 1999} \\
\hline Modell & $9 ; 20$ & 4,01 & 0,0047 & 1,51 & 0,2108 & 1,86 & 0,1190 & 5,49 & 0,0008 \\
\hline Regen & $2 ; 20$ & 10,59 & 0,0007 & 2,13 & 0,1445 & 2,98 & 0,0735 & 12,46 & 0,0003 \\
\hline Sonne & $1 ; 20$ & 1,21 & 0,2845 & 4,82 & 0,0402 & 0,08 & & 3,35 & 0,0823 \\
\hline $\mathrm{R} \times \mathrm{S}$ & 2,20 & 1,75 & 0,1997 & 0,42 & & 4,33 & 0,0274 & 6,20 & 0,0080 \\
\hline Block & $4 ; 20$ & 2,55 & 0,0713 & 0,92 & & 0,51 & & 2,18 & 0,1084 \\
\hline \multicolumn{10}{|c|}{ d) ANOVA Mai 2000} \\
\hline Modell & $9 ; 20$ & 8,10 & $<0,0001$ & 2,11 & 0,0783 & 3,62 & 0,0079 & 3,01 & 0,0192 \\
\hline Regen & $2 ; 20$ & 30,90 & $<0,0001$ & 0,36 & & 6,59 & 0,0063 & 4,44 & 0,0254 \\
\hline Sonne & $1 ; 20$ & 0,58 & & 4,09 & 0,0567 & 0,04 & & 2,98 & 0,0998 \\
\hline $\mathrm{R} \times \mathrm{S}$ & 2,20 & 1,39 & 0,2719 & 0,35 & & 8,06 & 0,0027 & 7,19 & 0,0044 \\
\hline Block & $4 ; 20$ & 1,94 & 0,1431 & 3,38 & 0,0289 & 0,82 & & 0,22 & \\
\hline
\end{tabular}


Tabelle 10: F-Werte und Wabrscheinlichkeiten für a-Fehler (p) der Behandlungs-, Block- und Termineffekte und deren Interaktionen aus zweifaktoriellen Varianzanalysen für Messwiederholungen der Bodenanalysen in den Nestbügeln der Gelben Wiesenameise. FG: Freiheitsgrade (Effekt; Fehler); innerbalb der Behandlungsstufen F-Werte des multivariaten Pillai trace Tests; $p<0,05$ : F-Werte fett gesetzt; $p>0,3$ nicht angegeben; V. Werte Wurzel-transformiert; Cmic: Mikrobielle Biomasse.

\begin{tabular}{|c|c|c|c|c|c|c|c|c|c|}
\hline & \multirow[b]{2}{*}{$\mathrm{FG}$} & \multicolumn{2}{|c|}{ Temperatur } & \multicolumn{2}{|c|}{ Wassergehalt } & \multicolumn{2}{|c|}{ Basalatmung ${ }^{\sqrt{ }}$} & \multicolumn{2}{|c|}{ Cmic } \\
\hline & & $\bar{F}$ & $\bar{p}$ & $\bar{F}$ & $\bar{p}$ & $\bar{F}$ & $\bar{p}$ & $\bar{F}$ & $\bar{p}$ \\
\hline Sonne & $1 ; 5$ & 29,06 & 0,0030 & 24,56 & 0,0077 & 0,03 & & 0,31 & \\
\hline Regen & $2 ; 5$ & 1,56 & 0,2980 & 14,98 & 0,0043 & 3,68 & 0,1041 & 0,85 & \\
\hline $\mathrm{R} \times \mathrm{S}$ & 2,5 & 1,05 & & 0,60 & & 2,88 & 0,1473 & 1,16 & \\
\hline Block & $1 ; 5$ & 4,81 & 0,0797 & 4,25 & 0,0942 & 2,52 & 0,1732 & 5,85 & 0,0602 \\
\hline Zeit & $2 ; 4$ & 868,67 & $<0,0001$ & 8,67 & 0,0351 & 6,73 & 0,0525 & 1,46 & \\
\hline $\mathrm{Z} \times \mathrm{S}$ & $2 ; 4$ & 2,41 & 0,2056 & 4,51 & 0,0945 & 2,26 & 0,2207 & 2,07 & 0,2413 \\
\hline $\mathrm{Z} \times \mathrm{R}$ & $4 ; 10$ & 1,14 & & 1,64 & 0,2387 & 0,79 & & 0,73 & \\
\hline $\mathrm{Z} \times \mathrm{S} \times \mathrm{R}$ & $4 ; 10$ & 0,53 & & 1,57 & 0,2562 & 0,44 & & 0,86 & \\
\hline $\mathrm{Z} \times \mathrm{B}$ & $2 ; 4$ & 2,41 & 0,2061 & 0,65 & & 2,59 & 0,1901 & 0,63 & \\
\hline
\end{tabular}

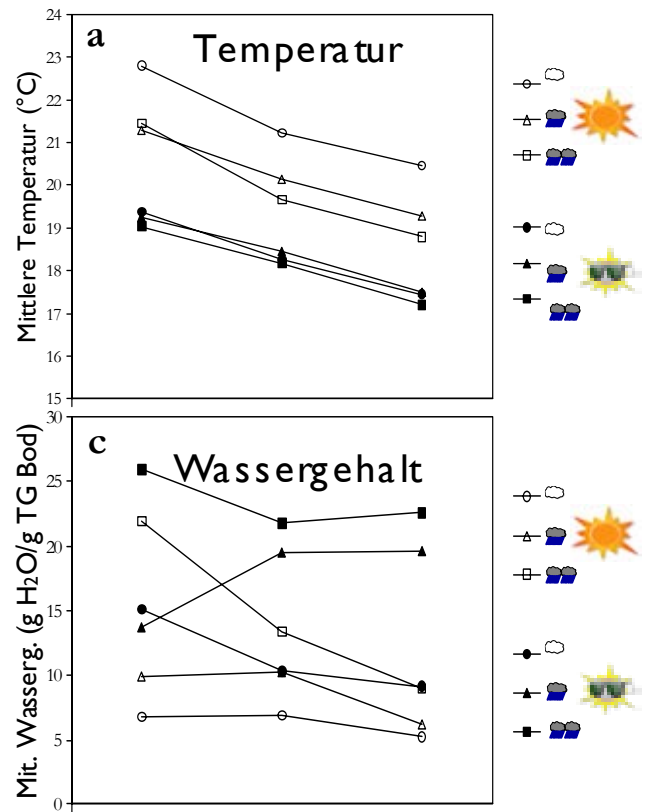

22. Aug. 31. Aug. 4. Sept.

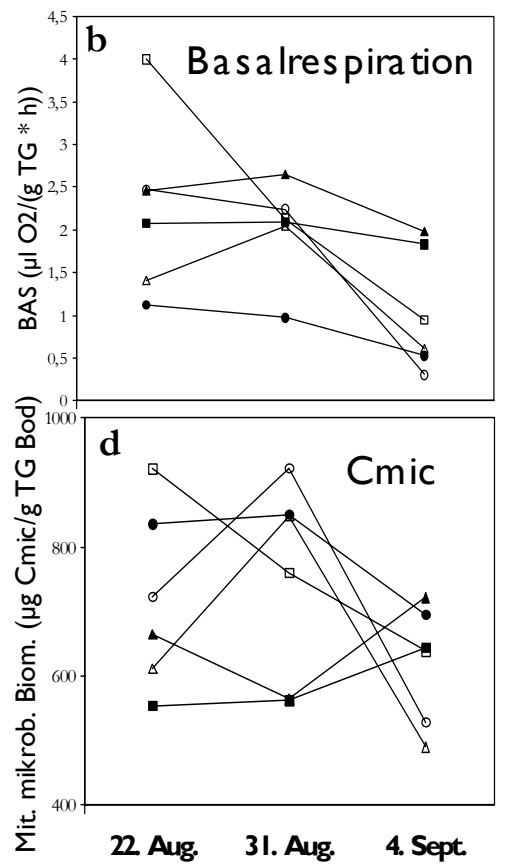

Abb. 26: Mittlere Temperatur in $5 \mathrm{~cm}$ Bodentiefe (a), Wassergehalt (c), Basalatmung (b) und mikrobielle Biomasse (d) im Sommer 2002 innerbalb der Nester von Lasius flavus unmittelbar, 9 und 13 Tage nach der letzten zusätzlichen Beregnung; Symbole sind in Abb. 24 erklärt: dunkle stehen für Beschattung, je mehr Ecken, um so mehr Niederschlag. 


\section{Mikroflora}

Der Faktor Regen hatte einen signifikanten Einfluss auf die Basalatmung des Bodens (Tab. 9), sie wird durch Niederschlagsminimierung und auch durch die zusätzliche Beregnung im Vergleich zur Kontrolle herabgesetzt. Die mikrobielle Biomasse wird vom Wassergehalt und der Menge der organischen Substanz signifikant beeinflusst. Wird der räumlich stark schwankende Corg-Gehalt als Kovariable berücksichtigt (ANCOVA), hat der Faktor Niederschlagsmenge auch einen signifikanten Einfluss auf die mikrobielle Biomasse.

\subsubsection{Auswirkungen auf die Bodentiere}

\section{Mikrofauna: Nematoda}

Die Dichten und Biomassen der Nematoden waren auf den besonnten Flächen ohne Niederschlag am geringsten. Auf den beschatteten trockenen Flächen waren die Dichten zu Beginn des Experiments am höchsten, nach zwei Jahren lagen sie immer noch auf dem Niveau der zusätzlich beregneten Flächen (Abb. 25 d). Insgesamt hat die Beschattung einen positiven Effekt auf die Fadenwürmer, auch wenn dieser nicht unabhängig von der Beregnung ist. Durch Beschattung stieg der Anteil der Bakterien- und Wurzelhaarfresser und mit dem Anteil der Kselektierten Gattungen auch der Maturity Index (Woltmann 2002).

\section{Mesofauna}

In den Nesthügeln hatte die Beschattung eine positive Wirkung auf die Springschwänze und die Hornmilben. Auf der Wiese war dieser Effekt nicht erkennbar (Abb. 27; Tittel, Platner \& Migge, unveröffentlicht).

Tabelle 11: F-Werte und Wabrscheinlichkeiten für $\alpha$-Fehler (p) der Behandlungseffekte und deren Interaktionen aus dreifaktoriellen Varianzanalysen (GLM, Typ III) der Mesofauna- und Ameisendichten von den Wiesenparzellen und aus den Nestern von Lasius flavus nach der Mikroklimamanipulation. $5 \mathrm{~cm} \varnothing$ Bohrkerne, Horizonte aufsummiert. FG: Freiheitsgrade (Effekt; Fehler); $p<0,05:$ F-Werte fett gesetzt; $p>0,3$ nicht angegeben; $V$ : Werte Quadratwurzel-

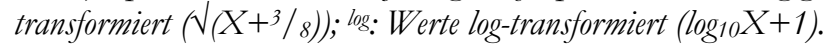

\begin{tabular}{|c|c|c|c|c|c|c|c|}
\hline & \multirow[b]{2}{*}{ FG } & \multicolumn{2}{|c|}{ Collembola $^{\sqrt{ }}$} & \multicolumn{2}{|c|}{ Acarina $\sqrt{ }$} & \multicolumn{2}{|c|}{ Formicidae $^{\log }$} \\
\hline & & $\bar{F}$ & $\overline{\mathrm{p}}$ & $\bar{F}$ & $\overline{\mathrm{p}}$ & $\bar{F}$ & $\bar{p}$ \\
\hline Modell & $11 ; 47$ & 7,00 & $<0,0001$ & 3,85 & 0,0006 & 9,09 & $<0,0001$ \\
\hline Regen & $2 ; 47$ & 2,66 & 0,0808 & 3,89 & 0,0273 & 1,23 & \\
\hline Sonne & $1 ; 47$ & 23,02 & $<0,0001$ & 6,87 & 0,0118 & 38,78 & $<0,0001$ \\
\hline Nest & $1 ; 47$ & 15,73 & 0,0002 & 0,95 & & 41,69 & $<0,0001$ \\
\hline Regen $\times$ Sonne & $2 ; 47$ & 4,04 & 0,0240 & 1,25 & 0,2947 & 1,20 & \\
\hline Regen $\times$ Nest & $2 ; 47$ & 1,39 & 0,2590 & 2,32 & 0,1099 & 1,35 & 0,2701 \\
\hline Sonne $\times$ Nest & $1 ; 47$ & 22,98 & $<0,0001$ & 14,48 & 0,0004 & 12,56 & 0,0009 \\
\hline $\mathrm{R} \times \mathrm{S} \times \mathrm{N}$ & $2 ; 47$ & 0,14 & & 2,55 & 0,0888 & 0,06 & \\
\hline
\end{tabular}


In den Kontrollvarianten war die Dichte der Collembola in den Nesthügeln von Lasius flavus deutlich niedriger als auf der Wiese; die Milben hingegen siedelten etwa in gleicher Zahl innerhalb und außerhalb der Erdnester.

Wenn infolge der Beschattung die Ameisenaktivität in den Hügeln auf das Niveau der umgebenden Wiese gesunken ist, bauen die Springschwänze hier sogar teilweise größere Populationen als im umgebenden Wiesenboden auf.

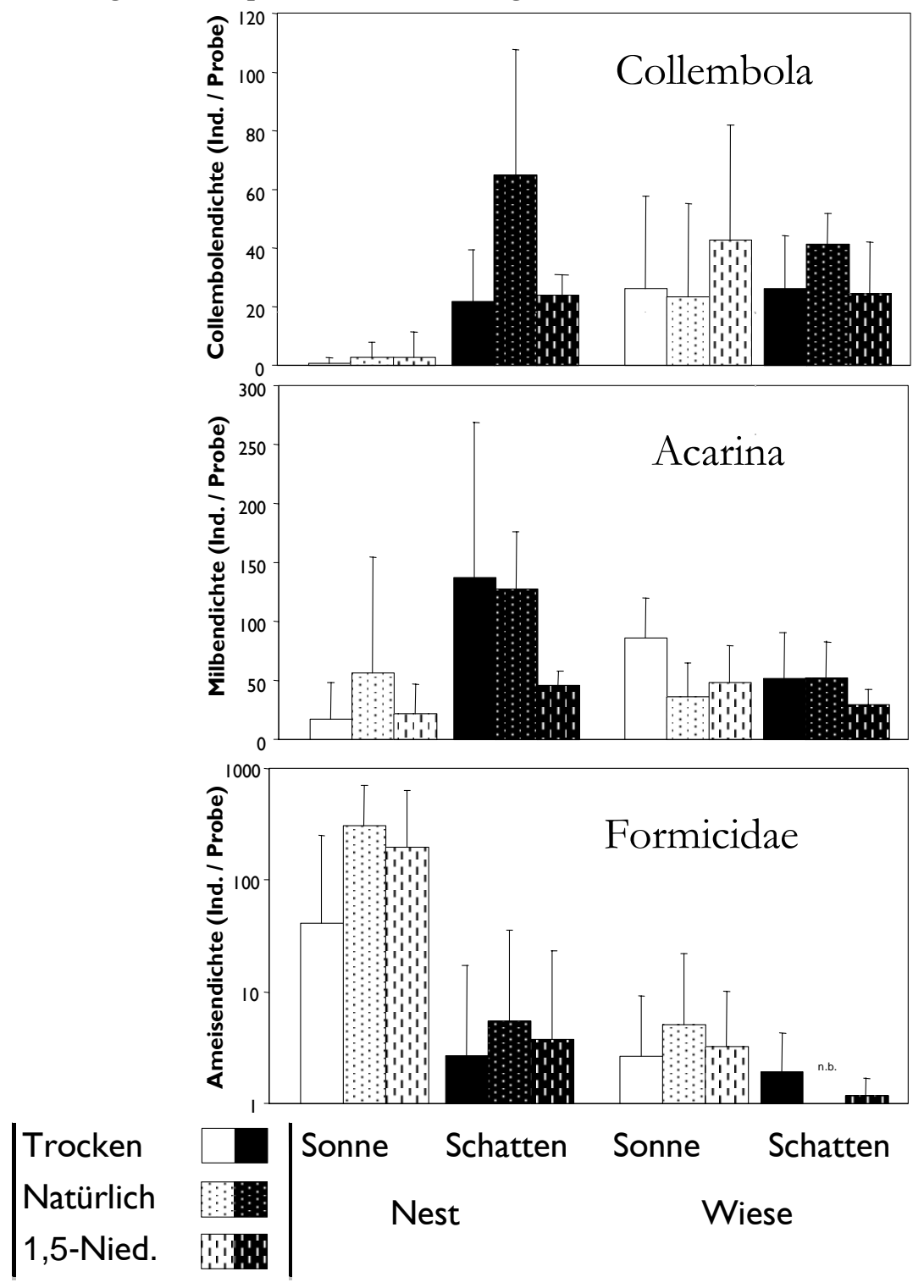


Abb. 27: Mittlere Individuenzablen der Springschwänze, Milben und Ameisen im Mai 1999 in den MacFadyen-Proben aus den Nestbügeln und von den Wiesenparzellen in den je 6 Varianten der Klimamanipulation mit Standardabweichungen; Skala der Ameisendichte logarithmisch.

\section{Saprophage und Pantophage}

Für die Dichten der Regenwürmer (Lumbricidae, v.a. Lumbricus castaneus und Octolasion lacteum) war der Faktor Regen signifikant (Tab. 12), sie traten im Sommer fast nur auf den feuchteren Flächen auf und erreichten dort im Frühjahr etwa viermal höhere Dichten als auf den trockenen Parzellen (Abb. 28).

Für alle anderen vorwiegend saprophagen Tiergruppen, die untersucht wurden, hatten beide manipulierte Faktoren, Regen und Sonne, einen signifikanten Einfluss auf die Individuendichten. Schnellkäferlarven (Elateridae) hatten die höchsten Dichten auf den besonnten, trockenen Flächen; Asseln (Isopoda, v.a. Philoscia muscorum, Hyloniscus riparius, Haplophthalmus mengii und Armadillidium spec.), Tausendfüßer (Diplopoda) und Schnecken (Gastropoda) hatten zu Beginn auf den jeweils feuchteren und schattigeren Flächen die höhere Dichten, nach zwei Jahren aber auf den feuchten und besonnten.
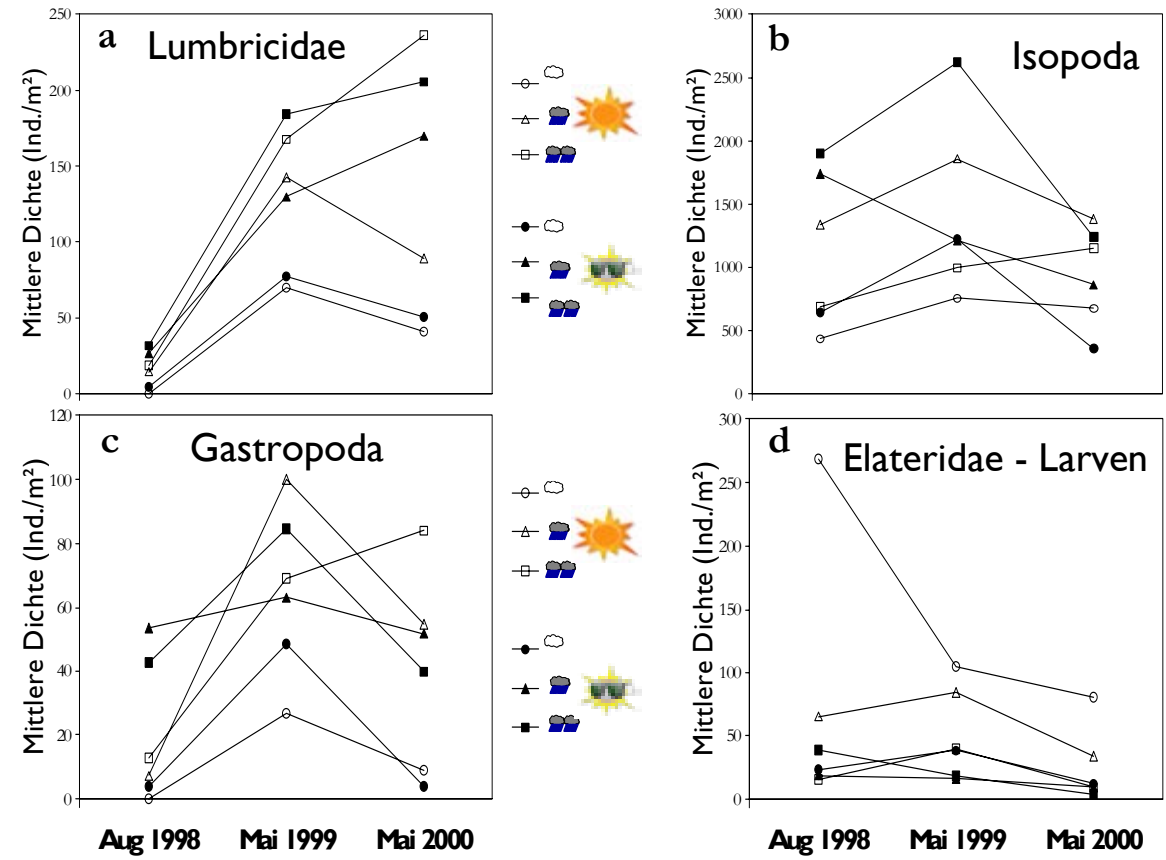

Aug 1998 Mai $1999 \quad$ Mai 2000

Abb. 28: Mittlere Siedlungsdichten in 0-5 cm Bodentiefe von Regenwürmern (a), Asseln (b), Schnecken (c) und Schnellkeäfer-Larven (d) auf den Wiesenparzellen 1/4, 1 und 2 Jahre nach Beginn der Mikroklimamanipulation; Symbole sind in Abb. 24 erklärt. 
Tabelle 12: F-Werte und Wabrscheinlichkeiten für $\alpha$-Fehler (p) der Behandlungs-, Block- und Termineffekte und deren Interaktionen aus zweifaktoriellen Varianzanalysen für Messwiederholungen der Individuendichten der Sapro- und Pantophaga von den Wiesenparzellen nach der Mikroklimamanipulation. FG: Freiheitsgrade (Effekt; Fehler); innerhalb der Behandlungsstufen FWerte des multivariaten Pillai trace Tests; $p<0,05: F$-Werte fett gesetst; $p$-Werte angegeben, wenn $p<0,3 ; \sqrt{ }$ : Werte Quadratwurzel-transformiert $(\sqrt{ }(X+3 / 8))$.

\section{a) repeated measurement}

\begin{tabular}{|c|c|c|c|c|c|c|c|c|c|}
\hline \multirow[t]{2}{*}{ 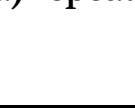 } & \multirow[b]{2}{*}{$\mathrm{FG}$} & \multicolumn{2}{|c|}{ Lumbricidae $^{\sqrt{ }}$} & \multicolumn{2}{|c|}{ Isopoda $^{\sqrt{ }}$} & \multicolumn{2}{|c|}{ Gastropoda $^{\sqrt{ }}$} & \multicolumn{2}{|c|}{ Elateridae $^{\sqrt{ }}$} \\
\hline & & $\mathrm{F}$ & $\mathrm{p}$ & $\mathrm{F}$ & $\mathrm{p}$ & $\mathrm{F}$ & $\mathrm{p}$ & $\mathrm{F}$ & $\mathrm{p}$ \\
\hline Regen & $2 ; 20$ & 29,51 & $<0,0001$ & 10,72 & 0,0007 & 10,16 & 0,0009 & 5,01 & 0,0173 \\
\hline Sonne & $1 ; 20$ & 1,79 & 0,1956 & 2,34 & 0,1421 & 0,46 & & 12,19 & 0,0023 \\
\hline $\mathrm{R} \times \mathrm{S}$ & 2,20 & 0,39 & & 4,33 & 0,0274 & 0,03 & & 3,70 & 0,0428 \\
\hline Block & $1 ; 20$ & 0,59 & & 8,71 & 0,0003 & 0,89 & & 1,66 & 0,1984 \\
\hline Zeit & $2 ; 19$ & 32,87 & $<0,0001$ & 7,42 & 0,0041 & 18,17 & $<0,0001$ & 3,59 & 0,0475 \\
\hline $\mathrm{Z} \times \mathrm{R}$ & $4 ; 40$ & 1,666 & 0,1792 & 1,16 & & 0,42 & & 0,52 & \\
\hline $\mathrm{Z} \times \mathrm{S}$ & $2 ; 19$ & 0,19 & & 4,83 & 0,0202 & 5,48 & 0,0132 & 0,29 & \\
\hline $\mathrm{Z} \times \mathrm{R} \times \mathrm{S}$ & $4 ; 40$ & 0,73 & & 1,56 & 0,2046 & 1,71 & 0,1669 & 1,60 & 0,1929 \\
\hline $\mathrm{Z} \times \mathrm{Bl}$ & $8 ; 40$ & 0,65 & & 0,99 & & 1,45 & 0,2060 & 0,75 & \\
\hline
\end{tabular}

b) ANOVA August 1998

\begin{tabular}{|c|c|c|c|c|c|c|c|c|c|}
\hline Modell & $9 ; 20$ & 1,29 & & 10,90 & $<0,0001$ & 4,96 & 0,0014 & 2,98 & 0,0201 \\
\hline Regen & $2 ; 20$ & 3,62 & 0,0455 & 15,16 & $<0,0001$ & 7,24 & 0,0043 & 3,67 & 0,0439 \\
\hline Sonne & $1 ; 20$ & 1,38 & 0,2536 & 13,10 & 0,0017 & 13,07 & 0,0017 & 6,12 & 0,0224 \\
\hline $\mathrm{R} \times \mathrm{S}$ & 2,20 & 0,04 & & 2,84 & 0,0820 & 2,14 & 0,1440 & 5,06 & 0,0167 \\
\hline Block & $4 ; 20$ & 0,72 & & 12,24 & $<0,0001$ & 3,20 & 0,0348 & 0,81 & \\
\hline \multicolumn{10}{|c|}{ c) ANOVA Mai 1999} \\
\hline Modell & $9 ; 20$ & 1,23 & & 4,90 & 0,0015 & 1,16 & & 2,29 & 0,0594 \\
\hline Regen & $2 ; 20$ & 4,43 & 0,0256 & 3,21 & 0,0619 & 1,94 & 0,1698 & 1,88 & 0,1793 \\
\hline Sonne & $1 ; 20$ & 0,02 & & 3,33 & 0,0831 & 0,02 & & 8,95 & 0,0072 \\
\hline $\mathrm{R} \times \mathrm{S}$ & 2,20 & 0,08 & & 5,60 & 0,0117 & 0,76 & & 0,59 & \\
\hline Block & $4 ; 20$ & 0,50 & & 5,78 & 0,0029 & 1,25 & & 1,67 & 0,1958 \\
\hline \multicolumn{10}{|c|}{ d) ANOVA Mai 2000} \\
\hline Modell & $9 ; 20$ & 4,12 & 0,0040 & 1,94 & 0,1047 & 1,92 & 0,1069 & 1,48 & 0,2230 \\
\hline Regen & $2 ; 20$ & 15,59 & $<0,0001$ & 4,24 & 0,0291 & 7,25 & 0,0043 & 1,59 & 0,2286 \\
\hline Sonne & $1 ; 20$ & 0,81 & & 1,49 & 0,2360 & 1,27 & 0,2722 & 3,28 & 0,0852 \\
\hline $\mathrm{R} \times \mathrm{S}$ & 2,20 & 1,33 & 0,2859 & 0,63 & & 0,56 & & 0,74 & \\
\hline Block & $4 ; 20$ & 0,62 & & 1,55 & 0,2268 & 0,10 & & 1,34 & 0,2898 \\
\hline
\end{tabular}


Phytophage und Pflanzen

Auf den besonnten, trockenen Flächen traten deutlich mehr Pflanzenläuse (Sternorrhyncha, v.a. Röhrenschildläuse (Ortheziidae)) und im August auch Fransenflügler (Thysanoptera) auf als auf den feuchteren und beschatteten Flächen (Abb. 29, Platner et al. 1999). Für die Dichte der Fransenflügler, Pflanzenläuse und Zikaden war dabei der Faktor Sonne signifikant, für die Dichte der Ortheziidae zunächst die Niederschlagsmenge und später auch die Sonneneinstrahlung (Tab. 13).

Im Laufe des Experiments reagierte die Vegetation sehr deutlich auf die experimentellen Veränderungen (Tab. 14, Abb. 29 d): Nach Austrocknung konnte fast keine oberirdische Biomasse mehr gebildet werden, nach zusätzlicher Beregnung konnte mit weniger Wurzeln mehr Sprossmasse aufgebaut werden. Auf beschatteten Parzellen war die Erdoberfläche nach zwei Jahren nur noch von Moos und bei zusätzlicher Austrocknung auch von Streu bedeckt. In den feuchteren Schattenparzellen war auch die Streuauflage fast vollständig verschwunden, so dass hier große Anteile des Bodens blank lagen.
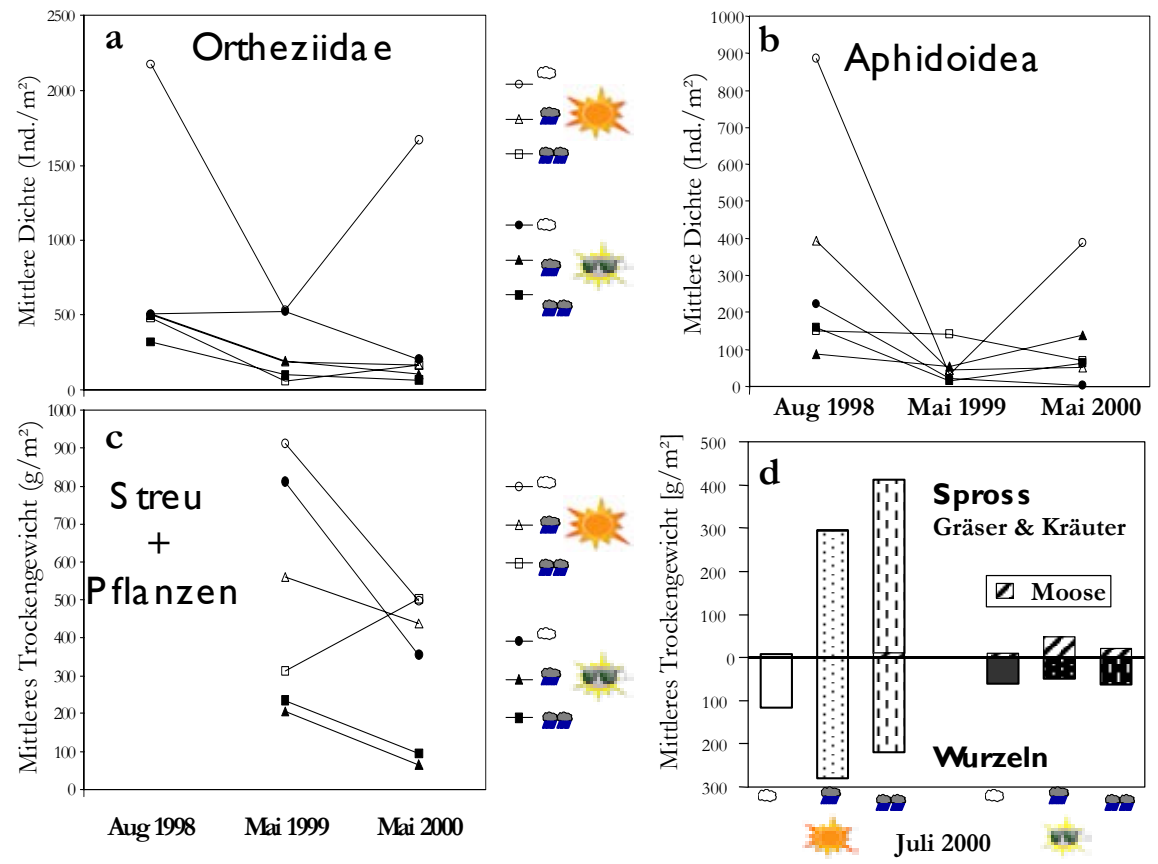

Abb. 29: Mittlere Siedlungsdichten von Röhrenschildläusen (a) und Blattläusen (b) in 0-5 cm Bodentiefe, Trockengewichte von Streu und Pflanzen (c, d) auf den Wiesenparzellen im zeitlichen Verlauf (a, b, c: 1/4, 1 und 2 Jahre nach Beginn der Mikroklimamanipulation) bzw. am Ende des Experiments (d: ober- und unterirdische Biomassen von Gefäßpflanzen und Moosen); Symbole vgl. Abb. 24 \& 27. 
Tabelle 13: F-Werte und Wabrscheinlichkeiten für $\alpha$-Fehler (p) der Behandlungs-, Block- und Termineffekte und deren Interaktionen aus zweifaktoriellen Varianzanalysen für Messwiederholungen der Individuendichten der Phytophaga und der Trockengewichte von Streu und Pflanzen auf den Wiesenparzellen nach der Mikroklimamanipulation. FG: Freiheitsgrade (Effekt; Febler); innerbalb der Behandlungsstufen F-Werte des multivariaten Pillai trace Tests; $p<0,05: F$-Werte fett gesetzt; $p>0,3$ nicht angegeben; ${ }^{V}$ : Werte Quadratwurzel-transformiert $(\sqrt{ }(X+3 / 8))$.

a) repeated measurement

\begin{tabular}{|c|c|c|c|c|c|c|c|c|c|}
\hline & \multirow[b]{2}{*}{$\mathrm{FG}$} & \multicolumn{2}{|c|}{ Ortheziidae $^{\sqrt{ }}$} & \multicolumn{2}{|c|}{ Aphoidea $^{\sqrt{ }}$} & \multicolumn{2}{|c|}{$\begin{array}{l}\text { Auchenor- } \\
\text { rhyncha }\end{array}$} & \multicolumn{2}{|c|}{$\begin{array}{c}\text { Streu }+ \\
\text { Pflanzen }^{\sqrt{ }}\end{array}$} \\
\hline & & $\mathrm{F}$ & $\mathrm{p}$ & $\mathrm{F}$ & $\mathrm{p}$ & $\mathrm{F}$ & $\mathrm{p}$ & $\mathrm{F}_{\mathrm{FG}}$ & $\mathrm{p}$ \\
\hline Regen & $2 ; 20$ & 9,99 & 0,0010 & 1,47 & 0,2537 & 1,15 & & $\mathbf{1 6}, \mathbf{3 5 _ { 2 ; 2 0 }}$ & $<0,0001$ \\
\hline Sonne & $1 ; 20$ & 5,09 & 0,0354 & 8,38 & 0,0090 & 6,36 & 0,0203 & $\mathbf{2 5 ,} \mathbf{6 5}_{1 ; 20}$ & $<0,0001$ \\
\hline $\mathrm{R} \times \mathrm{S}$ & 2,20 & 3,09 & 0,0678 & 2,76 & 0,0874 & 0,73 & & $3,11_{2 ; 20}$ & 0,0669 \\
\hline Block & $4 ; 20$ & 1,83 & 0,1628 & 1,20 & & 1,32 & 0,2950 & $\mathbf{3}, \mathbf{3 9}_{4 ; 20}$ & 0,0285 \\
\hline Zeit & $2 ; 19$ & 11,42 & 0,0006 & 15,60 & $<0,0001$ & 0,14 & & $\mathbf{1 4 , 6 5 _ { 1 ; 2 0 }}$ & 0,0011 \\
\hline $\mathrm{Z} \times \mathrm{R}$ & $4 ; 40$ & 0,97 & & 2,38 & 0,0680 & 1,42 & 0,2458 & $\mathbf{4 , 0 9 _ { 2 ; 2 0 }}$ & 0,0324 \\
\hline $\mathrm{Z} \times \mathrm{S}$ & $2 ; 19$ & 10,26 & 0,0010 & 1,38 & 0,2763 & 5,68 & 0,0116 & $4,78_{1 ; 20}$ & 0,0408 \\
\hline $\mathrm{Z} \times \mathrm{R} \times \mathrm{S}$ & $4 ; 40$ & 3,07 & 0,0269 & 3,94 & 0,0086 & 2,35 & 0,0706 & $1,23_{2 ; 20}$ & \\
\hline $\mathrm{Z} \times \mathrm{Bl}$ & $8 ; 40$ & 1,65 & 0,1418 & 1,43 & 0,2150 & 0,57 & & $1,57_{4 ; 20}$ & 0,2202 \\
\hline \multicolumn{10}{|c|}{ b) ANOVA August 1998} \\
\hline Modell & $9 ; 20$ & 2,49 & 0,0423 & 2,06 & 0,0862 & 1,00 & & & \\
\hline Regen & $2 ; 20$ & 3,36 & 0,0550 & 2,92 & 0,0774 & 0,57 & & & \\
\hline Sonne & $1 ; 20$ & 3,59 & 0,0727 & 5,61 & 0,0280 & 0,00 & & & \\
\hline $\mathrm{R} \times \mathrm{S}$ & 2,20 & 2,35 & 0,1213 & 1,66 & 0,2153 & 3,16 & 0,0643 & & \\
\hline Block & $4 ; 20$ & 1,85 & 0,1592 & 0,93 & & 0,40 & & & \\
\hline \multicolumn{10}{|c|}{ c) ANOVA Mai 1999} \\
\hline Modell & $9 ; 20$ & 2,78 & 0,0272 & 0,81 & & 0,71 & & 5,57 & 0,0007 \\
\hline Regen & $2 ; 20$ & 6,77 & 0,0057 & 0,61 & & 0,64 & & 17,23 & $<0,0001$ \\
\hline Sonne & $1 ; 20$ & 0,04 & & 1,80 & 0,1943 & 1,75 & 0,2007 & 5,54 & 0,0289 \\
\hline $\mathrm{R} \times \mathrm{S}$ & 2,20 & 0,04 & & 1,56 & 0,2343 & 1,45 & 0,2576 & 1,67 & 0,2135 \\
\hline Block & $4 ; 20$ & 2,85 & 0,0511 & 0,29 & & 0,11 & & 1,69 & 0,1919 \\
\hline \multicolumn{10}{|c|}{ d) ANOVA Mai 2000} \\
\hline Modell & $9 ; 20$ & 7,36 & 0,0001 & 2,02 & 0,0919 & 5,97 & 0,0004 & 6,59 & 0,0002 \\
\hline Regen & $2 ; 20$ & 14,98 & 0,0001 & 0,39 & & 4,56 & 0,0234 & 4,24 & 0,0292 \\
\hline Sonne & $1 ; 20$ & 15,64 & 0,0008 & 1,89 & 0,1845 & 29,66 & $<0,0001$ & 129,93 & $<0,0001$ \\
\hline $\mathrm{R} \times \mathrm{S}$ & 2,20 & 7,23 & 0,0043 & 4,43 & 0,0256 & 0,46 & & 3,07 & 0,0690 \\
\hline Block & $4 ; 20$ & 1,55 & 0,2267 & 1,66 & 0,1997 & 3,51 & 0,0252 & 3,70 & 0,0206 \\
\hline
\end{tabular}


Tabelle 14: F-Werte und Wabrscheinlichkeiten für $\alpha$-Febler (p) der Behandlungs- und Blockeffekte und deren Interaktionen aus zweifaktoriellen Varianzanalysen der Trockengewichte von Moosen und der ober- und unterirdischen Teile von Gräsern und Kräutern auf den Wiesenparzellen im Mai 2000 nach zweijähriger Mikroklimamanipulation. FG: Freiheitsgrade (Effekt; Febler);

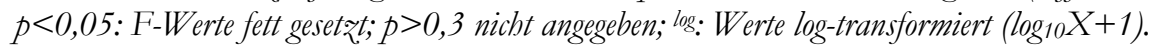

\begin{tabular}{|c|c|c|c|c|c|c|c|}
\hline & \multirow[b]{2}{*}{ FG } & \multicolumn{2}{|c|}{ Spross ${ }^{\log }$} & \multicolumn{2}{|c|}{ Wurzeln $n^{\log }$} & \multicolumn{2}{|c|}{ Moos $^{\log }$} \\
\hline & & $\mathrm{F}$ & $p$ & $\mathrm{~F}$ & $p$ & $\mathrm{~F}$ & $p$ \\
\hline Modell & $9 ; 20$ & 37,83 & $<0,0001$ & 1,75 & 0,1421 & 3,82 & 0,0060 \\
\hline Regen & $2 ; 20$ & 26,94 & $<0,0001$ & 0,40 & & 4,46 & 0,0250 \\
\hline Sonne & $1 ; 20$ & 213,57 & $<0,0001$ & 11,63 & 0,0028 & 10,36 & 0,0043 \\
\hline $\mathrm{R} \times \mathrm{S}$ & 2,20 & 34,89 & $<0,0001$ & 0,76 & & 1,60 & 0,2258 \\
\hline Block & $4 ; 20$ & 0,80 & & 0,45 & & 2,98 & 0,0441 \\
\hline
\end{tabular}

\section{Zoophage}

Die hochdominanten Hundertfüßer (Chilopoda, mittlere Dichte: 650 Ind./m²) zeigten zunächst keine deutlichen Unterschiede in der flächenbezogenen Zahl der Individuen, traten nach zwei Jahren aber auf den trockeneren Flächen signifikant häufiger auf. Auch für die Spinnen (Araneida), deren mittlere Dichte nach drei Monaten zwar auf den besonnten trockenen Flächen mit 970 Ind./ $\mathrm{m}^{2}$ mehr als doppelt so hoch wie auf den zusätzlich begossenen Flächen war, konnte anfangs kein signifikanter Einfluss nachgewiesen werden. Mit der Veränderung der Vegetationsstruktur aber war ihre Dichte auf den besonnten Flächen signifikant höher (Tab. 15).

Auf die Dichten der teilweise auch pantophagen Ameisen (Formicidae, va. Lasius flavus, L. niger und Myrmica rubra) und Weberknechte (Opilionida, überwiegend Fadenkanker (Nemastomatidae)) hatte die Beschattung jedoch einen hochsignifikanten Einfluss: Ameisen traten auf den besonnten Flächen, die Weberknechte dagegen auf den beschatteten und feuchteren Flächen in jeweils deutlich höheren Dichten auf. Für die Weberknechte kehrte sich der Effekt der Beschattung im Versuchsverlauf jedoch um (signifikante Interaktion Sonne $\times$ Zeit); im Mai 2000 hatten sie wie die Spinnen und Ameisen auf den besonnten Parzellen mit strukturreicher Kraut- und Streuschicht die höchsten Dichten, sie mieden allerdings weiterhin die trockenen Parzellen. 
Tabelle 15: F-Werte und Wabrscheinlichkeiten für $\alpha$-Fehler (p) der Behandlungs-, Block- und Termineffekte und deren Interaktionen aus zweifaktoriellen Varianzanalysen für Messwiederholungen der Individuendichten der Zoophaga von den Wiesenparzellen nach der Mikroklimamanipulation. FG: Freiheitsgrade (Effekt; Fehler); innerhalb der Behandlungsstufen F-Werte des multivariaten Pillai trace Tests; $p<0,05$ : F-Werte fett gesetzt; $p>0,3$ nicht angegeben; $V$ : Werte

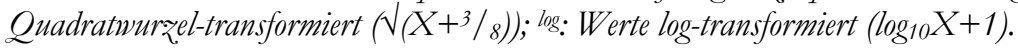

a) repeated measurement

\begin{tabular}{|c|c|c|c|c|c|c|c|c|c|}
\hline & \multirow[b]{2}{*}{$\mathrm{FG}$} & \multicolumn{2}{|c|}{ Formicidae $^{\log }$} & \multicolumn{2}{|c|}{ Chilopoda $^{\sqrt{ }}$} & \multicolumn{2}{|c|}{ Araneida $^{\sqrt{ }}$} & \multicolumn{2}{|c|}{ Opilonida $^{\sqrt{ }}$} \\
\hline & & $\mathrm{F}$ & $\mathrm{p}$ & $\mathrm{F}$ & $\mathrm{p}$ & $\mathrm{F}$ & $\mathrm{p}$ & $\mathrm{F}$ & $\mathrm{p}$ \\
\hline Regen & $2 ; 20$ & 0,31 & & 9,35 & 0,0014 & 1,52 & 0,2427 & 11,49 & 0,0005 \\
\hline Sonne & $1 ; 20$ & 27,91 & $<0,0001$ & 3,00 & 0,0984 & 8,66 & 0,0080 & 0,33 & \\
\hline $\mathrm{R} \times \mathrm{S}$ & 2,20 & 1,04 & & 2,25 & 0,1318 & 0,94 & & 4,72 & 0,0210 \\
\hline Block & $1 ; 20$ & 2,43 & 0,0814 & 1,56 & 0,2228 & 1,55 & 0,2259 & 1,89 & 0,1518 \\
\hline Zeit & $2 ; 19$ & 1,26 & & 6,24 & 0,0083 & 20,90 & $<0,0001$ & 6,60 & 0,0067 \\
\hline $\mathrm{Z} \times \mathrm{R}$ & $4 ; 40$ & 1,62 & 0,1879 & 2,40 & 0,0657 & 0,97 & & 2,16 & 0,0916 \\
\hline $\mathrm{Z} \times \mathrm{S}$ & $2 ; 19$ & 0,31 & & 2,57 & 0,1032 & 1,28 & & 27,20 & $<0,0001$ \\
\hline $\mathrm{Z} \times \mathrm{R} \times \mathrm{S}$ & $4 ; 40$ & 1,64 & 0,1823 & 0,42 & & 0,96 & & 4,29 & 0,0055 \\
\hline $\mathrm{Z} \times \mathrm{Bl}$ & $8 ; 40$ & 0,75 & & 1,87 & 0,0925 & 0,97 & & 3,07 & 0,0087 \\
\hline \multicolumn{10}{|c|}{ b) ANOVA August 1998} \\
\hline Modell & $9 ; 20$ & 2,55 & 0,0390 & 1,59 & 0,1839 & 1,78 & 0,1357 & 14,26 & $<0,0001$ \\
\hline Regen & $2 ; 20$ & 0,13 & & 0,05 & & 2,29 & 0,1269 & 11,96 & 0,0004 \\
\hline Sonne & $1 ; 20$ & 13,74 & 0,0014 & 0,13 & & 0,88 & & 47,02 & $<0,0001$ \\
\hline $\mathrm{R} \times \mathrm{S}$ & 2,20 & 0,24 & & 1,79 & 0,1923 & 0,93 & & 17,49 & $<0,0001$ \\
\hline Block & $4 ; 20$ & 2,11 & 0,1167 & 2,64 & 0,0645 & 2,17 & 0,1092 & 5,60 & 0,0034 \\
\hline \multicolumn{10}{|c|}{ c) ANOVA Mai 1999} \\
\hline Modell & $9 ; 20$ & 2,48 & 0,0439 & 2,09 & 0,0813 & 1,27 & & 0,94 & \\
\hline Regen & $2 ; 20$ & 0,18 & & 5,85 & 0,0100 & 0,13 & & 0,82 & \\
\hline Sonne & $1 ; 20$ & 8,77 & 0,0077 & 0,07 & & 2,96 & 0,1006 & 0,70 & \\
\hline $\mathrm{R} \times \mathrm{S}$ & 2,20 & 5,21 & 0,0151 & 0,75 & & 2,32 & 0,1241 & 0,43 & \\
\hline Block & $4 ; 20$ & 0,68 & & 1,39 & 0,2721 & 0,88 & & 1,31 & 0,2985 \\
\hline \multicolumn{10}{|c|}{ d) ANOVA Mai 2000} \\
\hline Modell & $9 ; 20$ & 2,32 & 0,0562 & 5,12 & 0,0012 & 1,49 & 0,2175 & 3,37 & 0,0113 \\
\hline Regen & $2 ; 20$ & 3,16 & 0,0643 & 13,82 & 0,0002 & 0,20 & & 5,84 & 0,0101 \\
\hline Sonne & $1 ; 20$ & 9,73 & 0,0054 & 11,35 & 0,0031 & 12,19 & 0,0023 & 8,34 & 0,0091 \\
\hline $\mathrm{R} \times \mathrm{S}$ & 2,20 & 0,03 & & 1,03 & & 0,27 & & 1,05 & \\
\hline Block & $4 ; 20$ & 1,19 & & 1,25 & & 0,07 & & 2,06 & 0,1246 \\
\hline
\end{tabular}




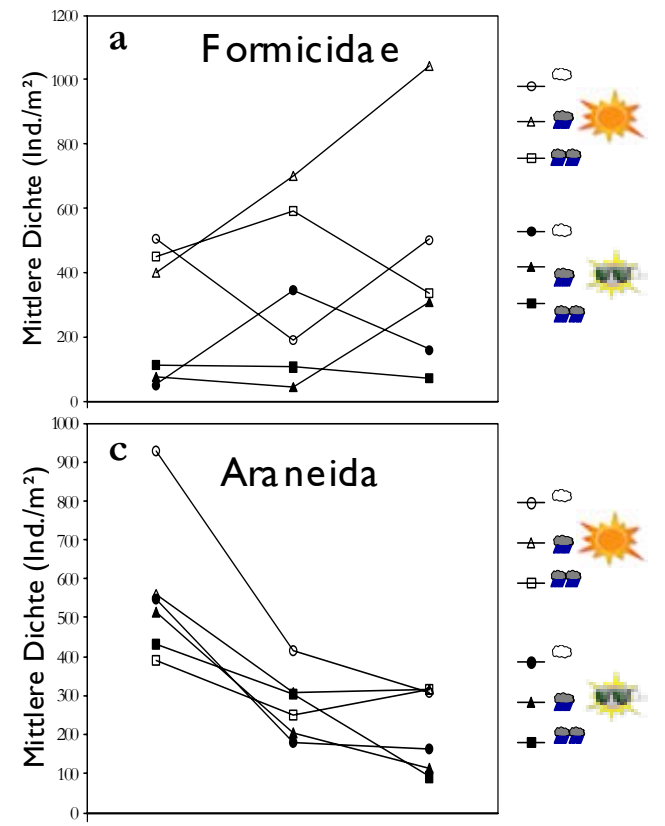

Aug 1998 Mai $1999 \quad$ Mai 2000
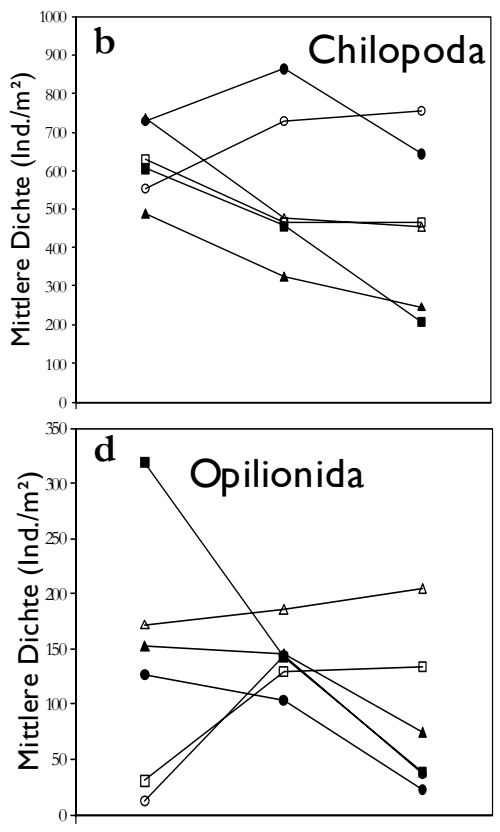

Aug 1998 Mai $1999 \quad$ Mai 2000

Abb. 30: Mittlere Siedlungsdichten in 0-5 cm Bodentiefe von Ameisen (a), Hundertfüßern (b), Spinnen (c) und Weberknechten (d) auf den Wiesenparzellen 1/4, 1 und 2 Jahre nach Beginn der Mikroklimamanipulation; Symbole sind in Abb. 24 erklärt.

\subsubsection{Zeitliche Unterschiede in der Wirkung auf die Bodentiergemein- schaft}

Vor Beginn der experimentellen Veränderungen war die Tiergemeinschaft auf den Teilbereichen der Glatthaferwiese ähnlich zusammengesetzt, ein schwacher Gradient trennt zwischen den waldrandnahen Parzellen und den im Winterhalbjahr kurzzeitig beschatteten Parzellen im unteren Hangbereich vor der Hecke (Abb. 31 a). Die Umweltparameter korrelieren nur sehr schwach mit den Tierdaten, der Eigenwert der ersten Achse sinkt von 0,206 der PCA auf 0,071 der RDA.

Nach Beginn der Manipulation verstärken sich die Unterschiede in der Verteilung der Tiere in den ersten drei Monaten nur wenig, der Eigenwert der ersten Achse einer PCA steigt auf 0,296. Allerdings korrelieren die Tierdaten erheblich besser mit den analysierten Umweltparametern, der Eigenwert bleibt in der RDA bei 0,217. Die erste Achse kennzeichnet jetzt den Unterschied zwischen den besonnten, trockenen Flächen mit Ameisen, Schnellkäferlarven, Thripsen und Röhrenschildläusen und den feuchteren, beschatteten mit Regenwürmern, Doppelfüßlern, Weberknechten, Asseln und Schnecken. Der Zentroid der WaldrandParzellen liegt immer noch getrennt von den anderen Blöcken, nun allerdings durch die zweite Achse (Abb. 31 b). 


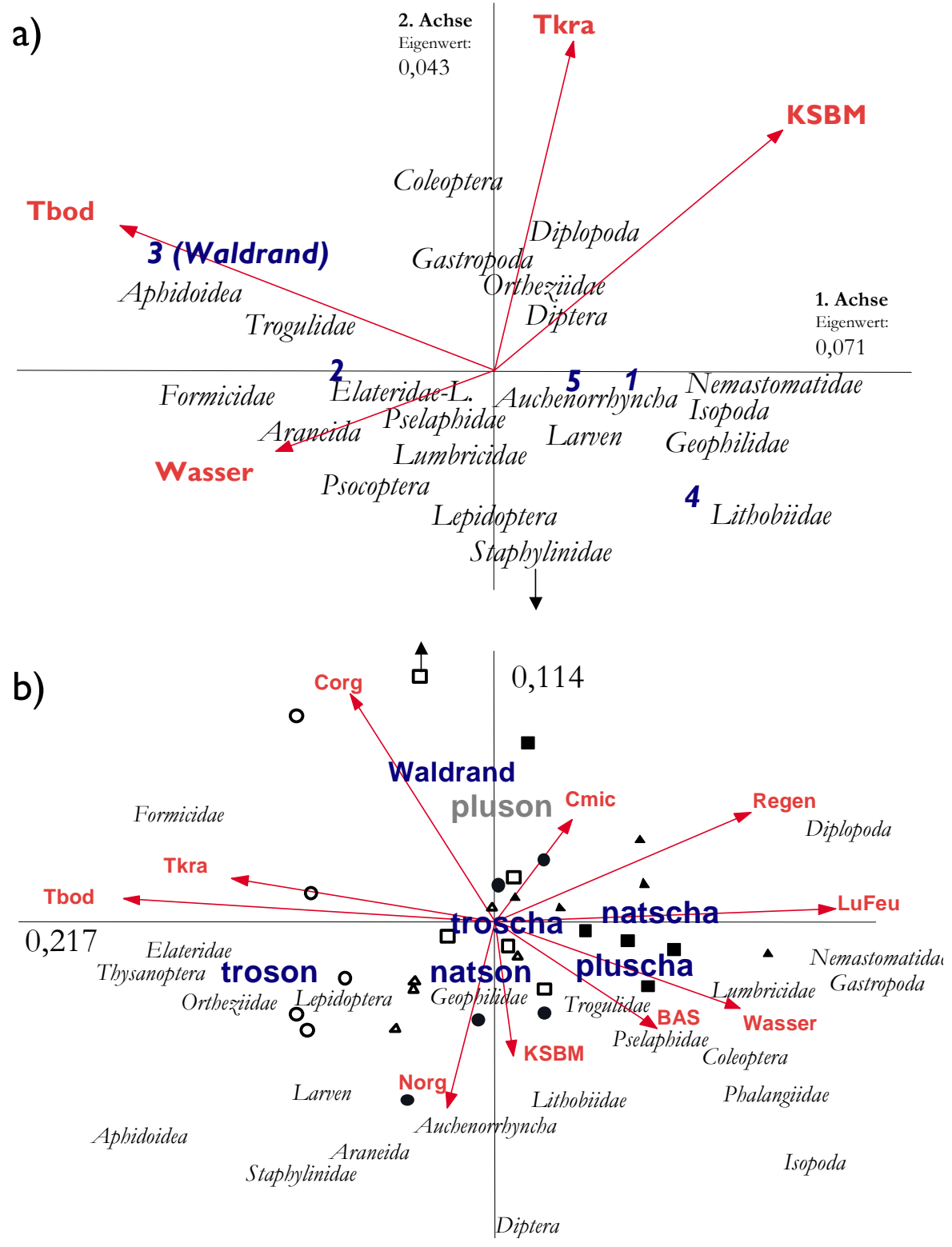

Abb. 31: Ordination der Siedlungsdichte verschiedener Tiertaxa im April 1998 (a) und im August 1998 (b), vor bzw. drei Monate nach Beginn der Mikroklimamanipulation, entlang der beiden ersten Achsen einer Redundanzanalyse (RDA). Die verschiedenen Blöcke (a) bzw. Behandlungen (b) sind als passive Variablen aufgetragen; tro-: trocken, nat-: natürlicher Niederschlag, plus-: zusätzlich beregnet; -son: besonnt, -scha: beschattet; Legende der Symbole für die Parzellenpunkte in Abb. 24; Coleoptera: weitere, überwiegend saprophage Käferfamilien; Elateridae: Schnellkäfer-Larven; Larven: Larven anderer Käferfamilien; Lepidoptera: Schmetterlingsraupen; Abkürzungen der Umweltvariablen in Abb. 32. 
a)

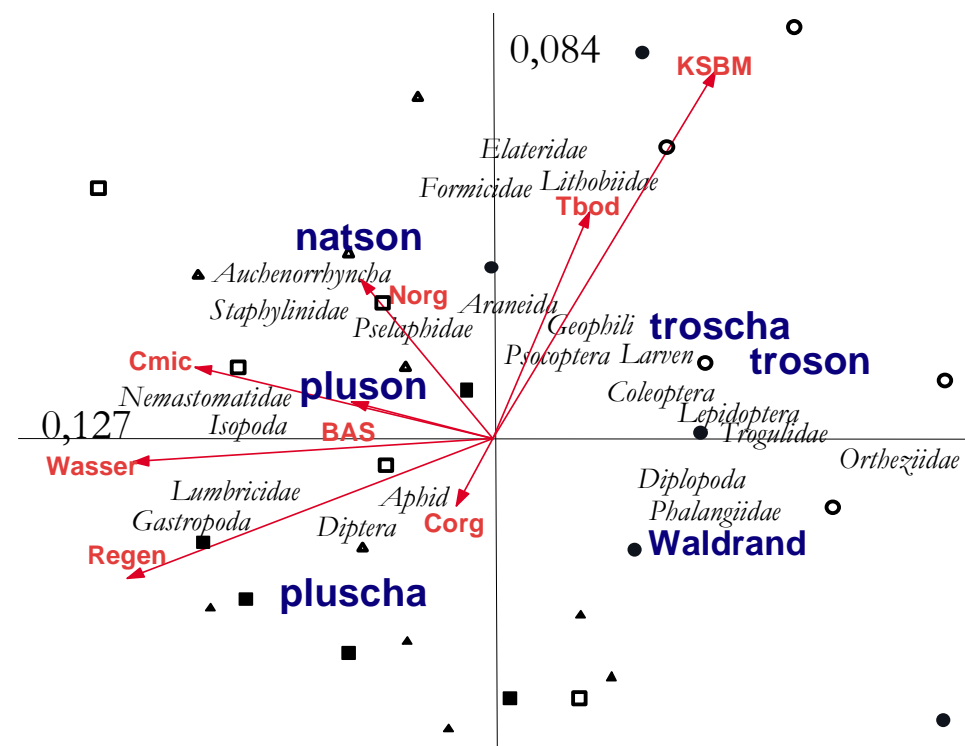

\section{natscha}

b)

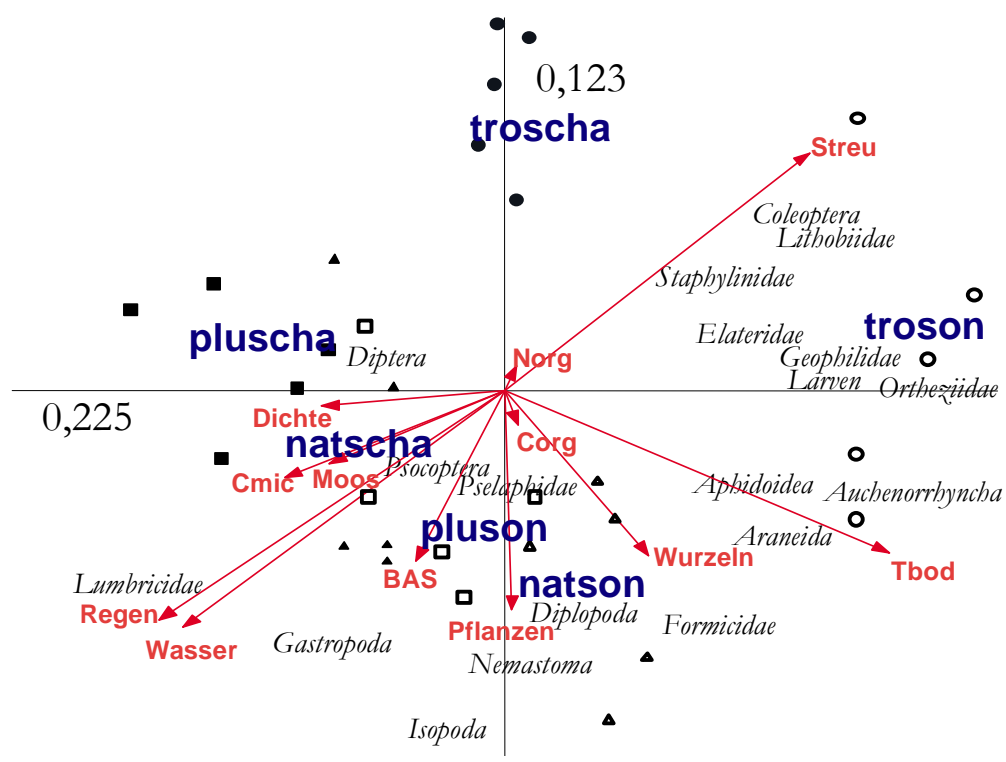

Abb. 32: Ordination der Siedlungsdichte verschiedener Tiertaxa im Mai 1999 (a) und Mai 2000 (b), 12 bzw. 24 Monate nach Beginn des Experiments, entlang der beiden ersten Achsen einer Redundanzanalyse (RDA). Die verschiedenen Behandlungen sind als passive Variablen aufgetragen; Legende der Symbole für die Parzellenpunkte in Abb. 24; Abkürzungen der Tiergruppen und Bebandlungen in Abb. 31; T: Mittlere Temperatur in $5 \mathrm{~cm}$ Bodentiefe (Tbod) brw. in der Krautschicbt (Tkra); Corg/Norg: Gesamtgehalt an Koblen-bzw. Stickstoff im Boden; Cmic: Mikrobielle Biomasse; BAS: Basaltmung; LuFeu: rel. Luffenchte; KSBM: Biomasse der Krautschicht bzw. der lebenden „Pflanzen", „W urzeln" und des „Moos"es; Regen: rel. Menge an Niederscblag. 
Nach einem Jahr verwischen sich die Differenzen zwischen den Tiergruppen etwas, der Eigenwert der ersten Achse einer PCA sinkt auf 0,205, eine Redundanzanalyse erklärt mit der ersten Achse nur noch 12,7 \% der Varianz (Abb. 32 a). Diese trennt jetzt in erster Linie die Parzellen mit vermindertem Niederschlag und die am Waldrand liegenden von den zusätzlich beregneten, feuchteren Parzellen und damit Doppelfüßler, Röhrenschildläuse und die Larven von Käfern und Schmetterlingen von den Regenwürmern, Schnecken und Asseln. Die zweite Achse korreliert mit dem Temperatur- und Streuauflage-Gradienten. Vor allem Ameisen, Hundertfüßler und Schnellkäferlarven haben bei höherer Bodentemperatur auch höhere Siedlungsdichten.

Nach zwei Jahren haben sich die Tiergemeinschaften auf den Versuchsparzellen wieder stärker (Eig. PCA: 0,259), aber deutlich anders als kurz nach Versuchsbeginn, differenziert. Die erste Achse trennt jetzt die trockenen, besonnten von den beregneten, beschatteten Parzellen und erklärt 22,5 \% der Varianz. Die zweite Achse trennt die trockenen, beschatteten von den unmanipulierten Parzellen und erklärt weitere 12,3 \% der Varianz. Die offenen, aber zusätzlich beregneten Parzellen werden zwischen die Kontroll- und die feuchteren Schatten-Parzellen ordiniert. Die verschiedenen Behandlungen bilden in der Auftragung sehr distinkte Cluster. Ameisen, Spinnen und Pflanzensaftsauger erreichen auf den besonnten Flächen mit hoher Bodentemperatur und Wurzelbiomasse ihre höchsten Siedlungsdichten, Asseln, Schnecken und Weber- knechte auf den Parzellen „ohne Dach“ mit den höchsten Pflanzenbiomassen, Regenwürmer auf den beregneten mit hohem Wassergehalt und mikrobieller Biomasse im Boden und die Steinläufer und verschiedene Käfer auf den trockenen Flächen mit hohem Streuanteil (Abb. 32 b). Die Blockunterschiede einschließlich des Waldrandeffekts traten jetzt nicht mehr so deutlich auf.

\subsubsection{Auswirkungen auf die Ameisengemeinschaft}

Die Ameisenarten unterschieden sich deutlich in ihren Siedlungsdichten auf den Versuchsparzellen, die erste Achse einer Korrelationsanalyse (DCA) hat einen Eigenwert von 0,464, der nach Hinzunahme der Umweltvariablen (CCA) auf 0,323 sinkt. Lasius flavus und Myrmica rubra hatten ihre höchsten Dichten an Arbeiterinnen auf den Parzellen mit den höchsten ober- und unterirdischen Pflanzenbiomassen. Stenamma debile und Myrmecina graminicola waren bevorzugt auf Flächen mit hohem Streuanteil aber niedriger Wurzelbiomasse $\mathrm{zu}$ finden. Lasius niger siedelte auch öfter auf Parzellen mit wenigen lebenden Pflanzen, aber eher in den dichteren, moosbewachsenen Bereichen.

Von den 15 beschatteten Nesthügeln von Lasius flavus wurden 11 verlassen und drei von Myrmica rubra besiedelt, von den besonnten Nestern nur in den ausgetrockneten und zusätzlich beregneten Parzellen jeweils ein Nest verlassen und eins von Lasius niger bzw. von Myrmica rubra besiedelt - alle anderen wurden noch von ihren Erbauerinnen bewohnt. Im Schatten konnte eine deutliche Abnahme des Volumens aller Nesthügel, abgeschätzt als ein Halbellipsoid, beobachtet werden. 


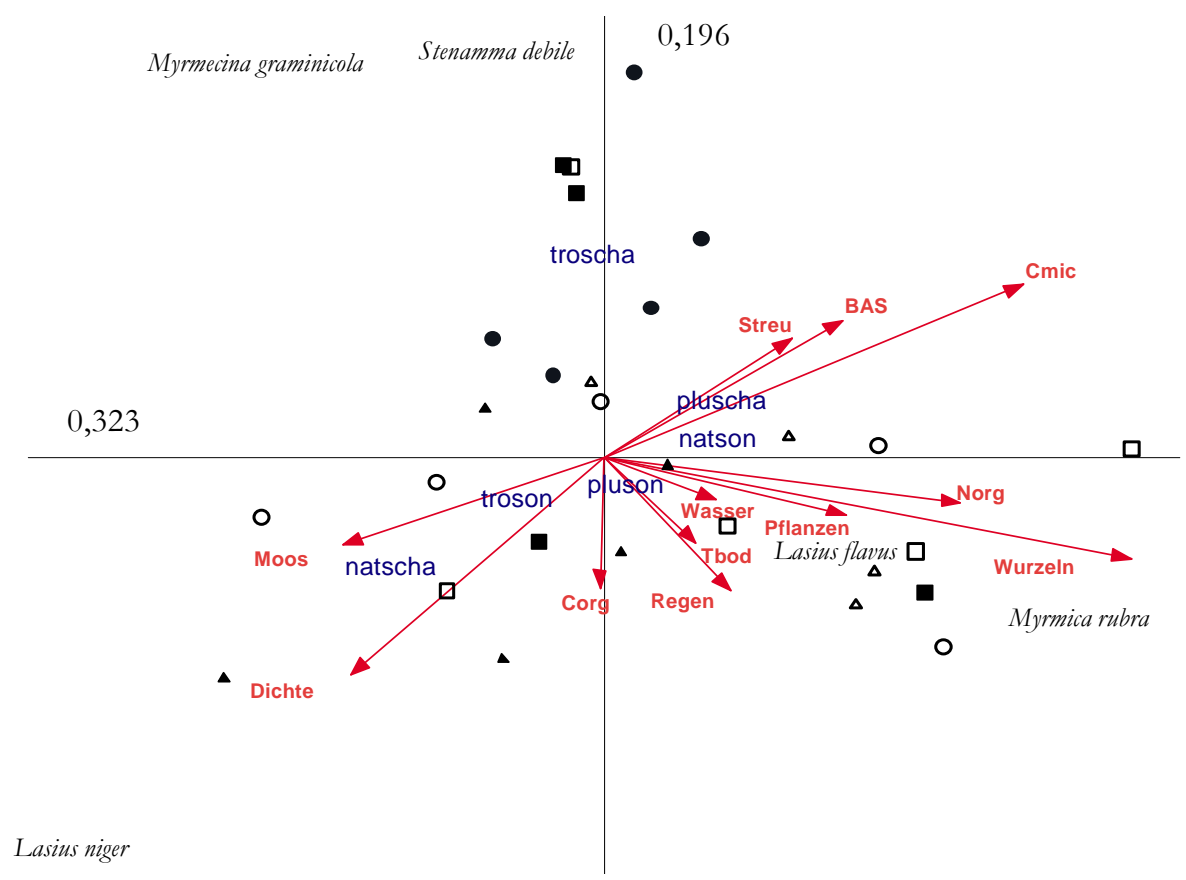

Abb. 33: Ordination der log-transformierten Arbeiterinnendichte der 5 bäufigsten Ameisenarten im Wiesenboden im Mai 2000, 24 Monate nach Beginn des Experiments, entlang der beiden ersten Achsen einer kanonischen Korrelationsanalyse (CCA). Die verschiedenen Behandlungen sind als passive Variablen aufgetragen (Abkürzungen bei Abb. 31 erlärt); Legende der Symbole für die Parzellenpunkte in Abb. 24, der Abkürzungen der Umweltvariablen in Abb. 32.

\subsection{Diskussion}

Offenlandbiotope mit ihren klimatischen Besonderheiten sind in Mitteleuropa typische Ameisenlebensräume. Sie hängen in ihrer Existenz oft von anthropogener Tätigkeit ab; Bewirtschaftung oder Brache mit anschließender natürlicher Sukzession sowie regionale Klimaverschiebungen können das Mikroklima z.T. erheblich verändern.

Die Boden-Mesofauna (Harte et al. 1996, Webb et al. 1998) und durch Dichteunabhängige Faktoren limitierte Phytophagen-Populationen (Whittaker \& Tribe 1998) können kurzfristig stark positiv auf eine experimentelle Temperaturerhöhung reagieren. Perfecto und Vandermeer (1996) testeten auf einer KaffeePlantage in Costa Rica die Auswirkungen von Schatten und Laubstreu auf die Ameisenfauna in einem zwei-faktoriellen Experiment: Die Abundanz der in unbeschatteten Anbausystemen dominanten Feuer-Ameise Solenopsis geminata nahm durch die Beschattung stark ab, die von anderen (Wald-) Ameisen-Arten stieg deutlich an. 
Frouz (2000) stellte beim Vergleich von verschiedenen Formica-Kolonien im Schatten fest, dass diejenigen der beschatteten Nester mit höherer Bodenfeuchte auch ausgeglichenere Temperaturverläufe und eine bessere Kolonieentwicklung erreichten. Den ausgeglicheneren Temperaturverlauf der zusätzlich beregneten und beschatteten Kolonien konnte ich auch beobachten. Aber wenn zusätzlich beregnet wurde, war die Ameisendichte nur kurzfristig tendenziell erhöht, mittelfristig hingegen sowohl inner- als auch außerhalb der Nester niedriger (Abb. 30 a). Bei Beregnung erreichten zwar die Boden-Temperaturen die größten Maxima, doch im Mittel lagen sie unter denen bei natürlichem Niederschlag. Die mikrobielle Biomasse stieg in besonnten Nestern nur unmittelbar nach der zusätzlichen Beregnung auf die höchsten Werte, sank dann aber zusammen mit dem Wassergehalt schnell wieder ab. Im Schatten war sie trotz des signifikant höheren Wassergehalts in der Tendenz niedriger als in besonnten Nestern. Mittel- und langfristig hatte also die zusätzliche Beregnung weder auf die Temperaturverhältnisse noch auf die Ameisen eine positive Wirkung.

Viele der beschatteten Erdhügel wurden von ihren Erbauerinnen verlassen und manche von ihnen von Myrmica rubra besiedelt, die offensichtlich besser mit der Beschattung leben kann (Elmes \& Wardlaw 1982). Werden alle acht als Arbeiterinnen nachgewiesenen Arten untransformiert mit einer DCA analysiert, ergibt sich für die erste Achse ein Eigenwert von 0,97, sie trennt v.a. Lasius alienus von den anderen Arten. Allerdings beeinflussen hier die Arten von wenigen Proben durch ihre hohen Abundanzen die multivariate Analyse viel zu stark, weshalb die logarithmierten Daten nur der konstanter auftretenden Arten belastbarere Aussagen zulassen. Die Schlüsselart auch außerhalb der Nesthügel ist die unterirdisch lebende Lasius flavus. Sie hängt trotz ihres Erdhügels sehr stark von genügender Sonneneinstrahlung ab. Wenn sie aufgrund einer lokalen Auskühlung, z.B. in Folge von Beschattung durch heranwachsende Gehölze, ihre Nester und unterirdischen Gänge verlassen muss, nutzen einige andere Ameisenarten und die Mesofauna bevorzugt diese Strukturen. Doch für die meisten Ameisen scheint das nur in eingeschränktem Maße möglich zu sein, viele Nesthügel werden ganz verlassen, wie auch die Untersuchung der verbuschten Bereiche auf Wiese 1 zeigte (Kap. 4).

Die experimentell veränderten Faktoren Niederschlagsmenge und Sonneneinstrahlung hatten auf die meisten der untersuchten Bodentiergruppen und wichtige biotische und abiotische Faktoren ihres Lebensraumes deutliche Auswirkungen. Die Tiere reagierten entsprechend ihrer Lebens- und Ernährungsweise unterschiedlich auf die Veränderungen des Mikroklimas: Die Pflanzensaft-saugenden Tiere (Phytophage) und die Larven der Elateridae hatten ihre höchsten Dichten auf den trockenen, besonnten Flächen, wohingegen die überwiegend saprophagen Tiere generell auf den beschatteten und stärker beregneten Flächen höhere Dichten hatten als auf den jeweils sonnigeren und trockeneren Flächen. Für die Schaumzikade Neophilaenus lineatus beispielsweise wurde eine starke positive Temperaturabhängigkeit ihrer Populationsdichten gefunden und experimentell bestätigt (Whittaker \& Tribe 1998). 
Auf Luzernefeldern hat Beschattung eine deutliche negative Wirkung auf die Phytophagen, sie war in einem Versuch der maßgebliche Umweltfaktor, der mehr als alle trophischen Kaskaden die Ökosystemprozesse veränderte (Dyer \& Stireman III 2003). In weniger intensiv bearbeiteten und damit auch diverseren Feldern wurde bei der Wiederholung des Versuchs die Nährstoffversorgung als Schlüsselfaktor festgestellt; allerdings wurden hier nur stark und schwach beschattete Varianten verglichen; da die offenen, besonnten Varianten nicht mehr angelegt waren.

Auch in einem Labor-Mikrokosmos-Experiment hatte Beschattung eine deutlichere Wirkung auf ober- und unterirdische Ökosystem-Komponenten als die zweimalige Entfernung der Hälfte aller Blätter von Nothofagus-Sämlingen (Mikola et al. 2000). Unterirdische Phytophage wie Ortheziidae auf den besonnten und Pflanzenparasitische Nematoden auf den beschatteten Flächen reagierten in unserem Versuch allerdings auch deutlich positiv auf die Niederschlagsminimierung.

In Agrarsystemen sind die ökologischen Bedingungen aber nur sehr eingeschränkt mit denen eines naturnahen Lebensraums vergleichbar. Auf einem Erbsenfeld stellten Frampton et al. (2000) in einem Versuch eine negative Wirkung von Austrocknung bzw. eine positive von Beregnung im Frühjahr auf alle Tiertaxa, unabhängig von ihrer Gildenzugehörigkeit fest, die bis in den Sommer hinein fortwirkte. Ameisen und Wurzelläuse, die in meinem Versuch positiv auf Austrocknung reagierten, kommen auf regelmäßig gepflügten Äckern beispielsweise nicht oder kaum vor.

Die Beutegreifer (Zoophage) zeigten unterschiedliche Muster in ihren Abundanzen: Chilopoda bevorzugten trockenere Bereiche und Araneida sonnigere; Formicidae (z.T. auch in Trophobiose mit Sternorryncha) reagierten ebenfalls negativ auf die Beschattung, Opilionida (z.T. auch saprophag) anfangs positiv. Im Laufe der zwei Jahre nahm die Dichte der Weberknechte in den beschatteten Parzellen jedoch deutlich ab, so dass sie mittelfristig nur noch in den beiden Behandlungen ohne Dächer, bei denen auch die Vegetation bessere Bedingungen hatte, Dichten von mehr als 100 Individuen pro Quadratmeter hatten. Im Unterschied zu den Spinnen, Ameisen und Hundertfüßern mieden die Weberknechte beide Austrocknungsvarianten.

Die Wassergehalte des Bodens veränderten sich erwartungsgemäß entsprechend der Behandlung. Die mikrobielle Biomasse wurde von der Niederschlagsmenge, dem $\mathrm{C}$ - und $\mathrm{H}_{2} \mathrm{O}-$ Gehalt positiv beeinflusst. Die C- und N-Gehalte unterliegen deutlich räumlichen Gradienten, wobei die Beschattung den C-Gehalt signifikant erniedrigte, vermutlich durch verringertes Pflanzenwurzelwachstum, was auch ein wichtiger Aspekt bei der Diskussion um $\mathrm{CO}_{2}-$ Senken sein kann (z.B. Clark 2004).

Die Saprophagen reagierten in diesem Experiment besonders auf zunehmende Feuchte positiv. Im Sommer drei Monate nach Beginn hatte auch die Beschattung einen positiven Effekt. Nach zwei Jahren traten alle saprophagen Taxa eher auf den besonnten Flächen häufiger auf. Das könnte zu einen an höheren Temperatur gelegen haben, die im Frühjahr in der besonnten Streu- und oberen Bodenschicht näher an das Optimum kommt (Wever et al. 2001), zum anderen an den 
Pflanzen und der Streuschicht, die auf den beschatteten, feuchten Parzellen nahezu komplett verschwunden war. Auf den beschatteten Parzellen ließ sich die Wirkung der Saprophagen sehr eindrucksvoll beobachten: auf denjenigen mit Niederschlag lag nach zwei Jahren der Boden bis auf etwas Moos blank, auf denjenigen ohne Niederschlag hatte sich eine mächtige Streuschicht akkumuliert, obwohl keine Pflanzen mehr wuchsen.

Wie in der deskriptiven Untersuchung (Kap. 4) hatten Spinnen, Ameisen und die Schnabelkerfe auch bei experimenteller Veränderung der Umweltbedingungen sehr ähnliche Muster. Sie repräsentieren ein typisches Phytophagen-basiertes Nahrungsnetz. Unter anderen mikroklimatischen Bedingungen, wie sie oft auch in Wäldern zu finden sind, dominiert ein deutlich stärker Saprophagen-basiertes Nahrungsnetz. In Folge der Beschattung wird eine Gruppe Ökosystemingenieure, die Ameisen, durch eine andere, die Regenwürmer, ersetzt. Das gesamte Nahrungsnetz gestaltet sich in Folge des Klimawandels stark um, was auch deutlich sichtbare Konsequenzen für alle Ökosystemprozesse hat. Da das Mikroklima als Komplex auf alle Glieder des Interaktionsnetzes einwirkt (Abb. 34), ist eine Trennung der einzelnen Wirkungspfade nicht möglich; der Gesamteinfluss des Mikroklimas auf das System ist aber in jedem Falle enorm.

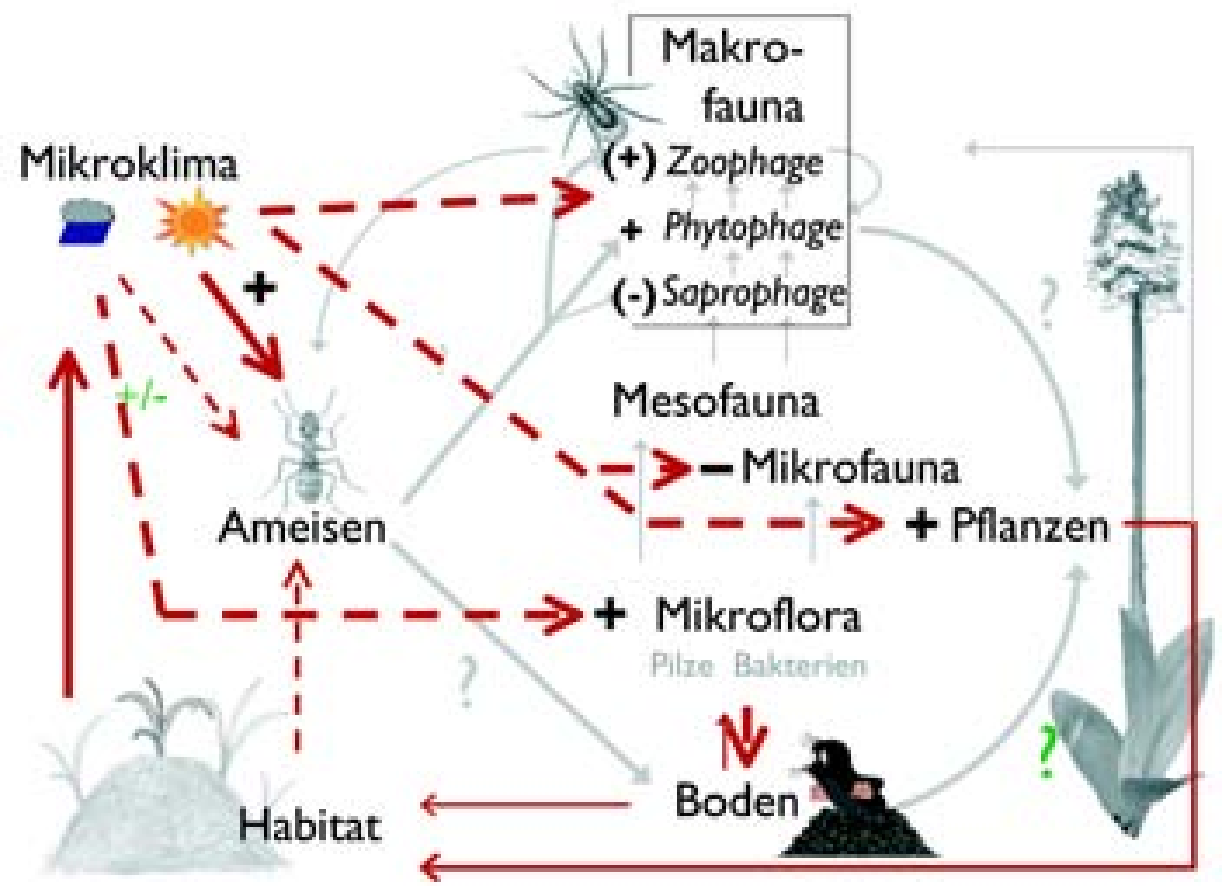

Abb. 34: Nachgewiesene Wirkungen des Mikroklimas: Sonneneinstrablung hat signifikante Auswirkungen auf Ameisen, die über deren Wechselwirkungen indirekt auch im Interaktionsnetz, weiterwirken, wirkt aber auch direkt positiv auf Pflanzen, Phytophage und einige Zoophage (Spinnen) sowie negativ auf Nematoden; Niederschlag wirkt positiv auf Mikroflora und Saprophage (gestrichelte Pfeile). 


\section{Themenbereich II \\ Stellung und Funktion der Ameisen im Nahrungsnetz}

\section{Nahrungsbiologie und Ermittlung der trophi- schen Ebene durch Analyse von stabilen Isotopen}

\subsection{Einleitung}

Ameisen als supraspezifisches Taxon mit mehr oder weniger omnivoren Vertretern sind nicht problemlos einer bestimmten trophischen Ebene zuzuordnen. Die Ernährungsgewohnheiten der wichtigen Arten auf Wiesen und Trockenrasen können sich relativ stark voneinander unterscheiden.

Für die unterirdisch lebende Art Lasius flavus wird angenommen, dass sie sich vorwiegend vom Honigtau der Wurzelläuse im Nestbereich ernährt (Czerwiński et al. 1971, Dauber \& Wolters 2000); die Tiere sind aber unterirdisch auch bis etwa 1 m im Umkreis stark aktiv (Pontin 1961, Waloff \& Blackith 1962) und können im Sommer die komplette Überproduktion der Wurzelläuse (mindestens 3000 erste Larvenstadien am Tag pro Nest) erbeuten und sie an ihre Larven verfüttern (Pontin 1978, Seifert 1996).

Die semi-prädatorische Art Lasius niger fouragiert sowohl an Wurzelläusen als auch stark in der Vegetationsschicht und trägt neben Honigtau und tierischer Beute auch Pflanzensamen ein (Czerwiński et al. 1971, Seifert 1996); zu Beginn der Wachstumssaison soll sie sich stärker von tierischer Kost ernähren und später überwiegend zu Honigtau wechseln (Pętal 1980, Elmes 1991).

Die stark prädatorischen Myrmica-Arten behalten hingegen das ganze Jahr über einen hohen Anteil an tierischer Kost (Czerwiński et al. 1971, Pętal 1980); das Verhältnis von Zoophagen zu Phytophagen in der Beute schwankt jährlich und ist 
dabei abhängig vom jeweiligen Beuteangebot - allerdings waren auf polnischen Wiesen Spinnen in der tatsächlichen Beute im Vergleich zur potentiellen Beute meist unter- und Zikaden meist überrepräsentiert (Pętal et al. 1971).

Auch die größeren Formica-Arten ernähren sich hauptsächlich zoophag und trophobiotisch (Seifert 1996), als größere Ameisen überwältigen sie auch größere Beute, während sie die kleinere Beute kleinerer Arten oft weitgehend ignorieren (Way \& Khoo 1992).

Die meisten heimischen Ameisenarten sind ausgesprochen omnivor, sie erbeuten große Mengen an Beutetieren, decken ihren hohen Energiebedarf aber oft weitgehend von zuckerhaltigen Flüssigkeiten. Das können Pflanzensäfte, Nektar und in hohem Maße der von Pflanzensaftsaugern ausgeschiedene Honigtau sein.

Bei steigender Versorgung durch Honigtau steigt auch der Bedarf an tierischer Kost, um die Nährstoff-Balance aufrechtzuerhalten, wie Pontin (1978) bei Lasius flavus nachwies; so wird die Populationsdichte und Alterstruktur der Läuse durch die Ameisen auf einem relativ konstanten Niveau gehalten.

Auch ein Freilandexperiment von Fraser \& Grime (1998), in dem eine positive top-down-Wirkung von Marienkäfern auf Gräser mit Blattlausbefall nur bei guter Nährstoffversorgung nachgewiesen werden konnte, macht deutlich, dass einzelne Wirkungen in komplexen Systemen immer auch von anderen Interaktionen abhängen. Doch gerade bei der Untersuchung der Interaktionen Pflanze-LausAmeise werden meist nur top-down-Wirkungen berücksichtigt (Buckley 1997a \& b, Morales 2000), auch die direkten Wirkungen der Ameisen auf die Trophobionten (Kosten und Nutzen) sind erst in letzter Zeit experimentell untersucht worden (Cushman \& Addicott 1989, Stadler \& Dixon 1998, Yao et al. 2000 und Morales 2000).

Nach dem Fretwell-Oksanen-Modell (Fretwell 1977, Oksanen et al. 1981) sind in Ökosystemen mittlerer Produktivität drei trophische Ebenen zu erwarten; die Zoophagen nutzen ihre Beute, die Phytophagen, dabei so stark, dass diese nicht mehr das Pflanzenwachstum durch Fraßdruck limitieren. Die Produzenten und Sekundärkonsumenten wären danach bottom-up-limitiert, die Primärkonsumenten hingegen top-down-kontrolliert.

Nach der Nährstoffbalance-Hypothese sollte eine zusätzliche Versorgung von Ameisen mit Kohlenhydraten zu stärkerem Fraßdruck auf die Blattläuse führen, deren Honigtau sie sonst stärker genutzt hätten. Nach dem Fretwell-OksanenModell würde eine zusätzliche Versorgung der Pflanzen positive Wirkungen auf die ganze Nahrungskette haben, zumindest solange keine Spitzenräuber dazu kommen. Wenn Pflanze und Läuse keine zusätzliche Ressourcen erhalten, sollte sich eine Zusatz-Fütterung von Ameisen solange neutral bis positiv auf die Läuse auswirken, bis sich die Ameisen durch die bessere Versorgung stärker vermehren und in der Folge einen stärkeren Fraßdruck ausüben können.

Eine experimentelle Überprüfung im tritrophischen System Pflanze-LausAmeise unter Berücksichtigung sowohl der bottom-up- als auch der top-downWirkungen erscheint hier besonders vielversprechend. Um die Relation beider 
Wirkungspfade bewerten zu können, müssen beide Wirkungen in einem gemeinsamen Experiment getestet werden.

Dazu mussten geeignete Versuchsgefäße entwickelt werden, die so flexibel gestaltet sein sollten, dass später auch andere Effekte, wie direkte bottom-up-Effekte durch die Ameisen selbst in ihrer Wirkung auf das tritrophische System getestet werden können.

Die Verhältnisse von stabilen Isotopen in Organismen können zur Analyse trophischer Beziehungen sowohl für Einzelorganismen als auch innerhalb ganzer Nahrungsnetze in terrestrischen und aquatischen Systemen benutzt werden (Rundel et al. 1989, Lajtha \& Michener 1994). In den meisten Fällen ist die Abweichung des ${ }^{13} \mathrm{C} /{ }^{12} \mathrm{C}$-Verhältnisses zwischen Tieren und ihrer Nahrung gering (DeNiro \& Epstein 1978); obwohl durch den Lipidstoffwechsel geringe Verschiebungen auftreten (Petelle et al. 1979), kann das ${ }^{13} \mathrm{C} /{ }^{12} \mathrm{C}$-Verhältnis doch oft noch die Kohlenstoffquelle widerspiegeln, insbesondere bei deutlichen Unterschieden, wie sie z.B. zwischen C-3- und C-4-Pflanzen auftreten (Peterson \& Fry 1987). Das ${ }^{15} \mathrm{~N} /{ }^{14} \mathrm{~N}-$ Verhältnis hingegen wird im Tier durchschnittlich um einen relativ konstanten Wert größer als das seiner Nahrung (im Mittel 3 bis $4 \%$ höhere $\delta^{15} \mathrm{~N}$ Werte), d.h. es kommt zu einer Anreicherung des schwereren Stickstoff-Isotops entlang von Nahrungsketten (DeNiro \& Epstein 1981, Minagawa \& Wada 1984), was eine Einordnung einzelner Tiere und Arten auf ein trophisches Niveau zulässt. Tayasu et al. (1997) konnten auf diese Weise die Ernährungsgewohnheiten von Termiten eingrenzen und Neilson et al. (1998) einige trophische Beziehungen im Boden einer Brachwiese klären. Ganze Boden-Nahrungsnetze wurden erst in den letzten Jahren in verschiedenen Wäldern umfassender untersucht (Ponsard \& Arditi 2000, Scheu \& Falca 2000), die ${ }^{15} \mathrm{~N} /{ }^{14} \mathrm{~N}-$ Verhältnisse reflektierten dabei adäquat die trophischen Strukturen der Gemeinschaften.

Die Analyse der natürlichen Verhältnisse stabiler Isotope scheint daher auch für Ameisengemeinschaften eine vielversprechende Methode zur Einordnung in trophische Ebenen zu sein, auch wenn sie bislang bei Ameisen nur in tropischen Regionen zur Charakterisierung von Stoffflüssen bei mutualistischen Beziehungen zwischen Ameisen und Ameisenpflanzen angewandt wurde (Fisher et al. 1990, Treseder et al. 1995, Sagers et al. 2000). Zudem erlaubt sie eine gezielte Beobachtung der Stoffflüsse in einem Experiment durch Zufütterung der Ameisen mit natürlich „markiertem“ Futter, also Futterquellen auf C4-Pflanzen-Basis.

\subsection{Methodik}

\subsubsection{Nahrungsnetzanalyse}

Die natürlichen Verhältnisse stabiler Stickstoff- und Kohlenstoff-Isotope von vier funktionellen Ameisengruppen mit unterschiedlicher Lebensweise (Lasius flavus, L. niger / alienus, Myrmica spec. und Formica spec.) wurden an verschiedenen Terminen vergleichend bestimmt. Dazu wurde im September 2002 mit verschiedenen Methoden (Bodenfallen, Saugapparat, Hand- und Kescherfänge) versucht, auf Wiese 
1 ein möglichst breites Spektrum des ganzen Nahrungsnetzes zu erfassen. Die Ameisenarbeiterinnen wurden dann zusammen mit Pflanzenproben und mit Arten anderer Tiergruppen, deren trophische Ebene z.T. bekannt ist, analysiert, was eine Einordnung in die trophischen Ebenen des Nahrungsnetzes ermöglicht. Als Basis des Nahrungsnetzes wurden die Pflanzen aus drei Bodenproben in Gräser, Kräuter und Moose sortiert, getrocknet, gemüllert und in mehreren Parallelen analysiert.

Zur Feststellung von Unterschieden der einzelnen funktionellen Gruppen und ihrer Entwicklungsstadien im Jahreslauf wurden am 10. April, 9. Juni und 4. August 2000 aus drei Nestern jeder Art von Wiese 1 und den Teilflächen 1b und 1c Mischproben an Arbeiterinnen und Eiern, Larven, Puppen bzw. Geschlechtstieren entnommen und direkt tiefgefroren.

Zur methodischen Verfeinerung wurden ergänzend Ameisen der Art Lasius niger, die auf einem benachbarten Wildacker leben, auf dem schon seit zehn Jahren Mais als C-4-Pflanze und Klee als Stickstofffixierer angebaut werden, zusammen mit anderen Tieren, Pflanzen- und Bodenproben analysiert.

Die genaue Behandlung des Tiermaterials und das Verfahren der Analyse der stabilen Isotope wurde in Kapitel 3 ausführlich beschrieben.

\subsubsection{Laborversuch}

Im Sommer 2003 wurde in eigens entwickelten Gefäßen (Abb. 35) je eine Buschbohne (Phaseolus vulgaris, Sorte Solido aus biol. Anbau, Hof Bohnhorst, Natendorf-Oldendorf) in 2,2 kg TG durch Hitzeextraktion defaunierten und gesiebten Boden gepflanzt, mit Schwarzen Bohnenläusen (Aphis fabae aus Zucht des Instituts für Pflanzenpathologie, Universität Göttingen) besetzt und in ihrem durch Gaze abgetrennten Wurzelraum ein Teil (50 Arbeiterinnen, 25 Puppen und 25 Larven) von im Freiland gesammelten Kolonien der Schwarzgrauen Wegameise (Lasius niger) angesiedelt. Da Lasius niger monogyn ist, standen nicht genügend Königinnen zur Verfügung, um dazugegeben zu werden.

Auf der Südseite jedes Topfes wurde ein flacher Kalkstein als Wärmespeicher auf die Erdoberfläche gelegt, auf der Nordseite wurde ein kleiner Tontopf mit Wattebausch eingegraben, der beim Bewässern Erosion verminderte und als Wasserreservoir einen Feuchtigkeitsgradienten im Erdboden aufbauen und halten konnte. Die Bodentemperatur wurde in Kontrollgefäßen ohne Ameisenkolonie regelmäßig geprüft. Das Optimum liegt für Lasius niger bei etwa $25{ }^{\circ} \mathrm{C}$ (Seifert 1986, Czechowski \& Pisarski 1992). Durch je einen Schlauch erhielten die Ameisen Zugang zum oberirdischen Teil jedes Kosmos mit den Läusen und zu einer separaten Futterkammer, in der ihnen in Schälchen mit Watte

(1) nur Wasser

(2) Wasser + Rohrzuckerlösung

(3) Wasser + Mehlwürmer

(4) Wasser + Rohrzuckerlösung + Mehlwürmer

angeboten wurden. Die Mehlwürmer (Tenebrio molitor) wurden zuvor über mehrere Generationen in Maismehl und Maisgries angezogen. 


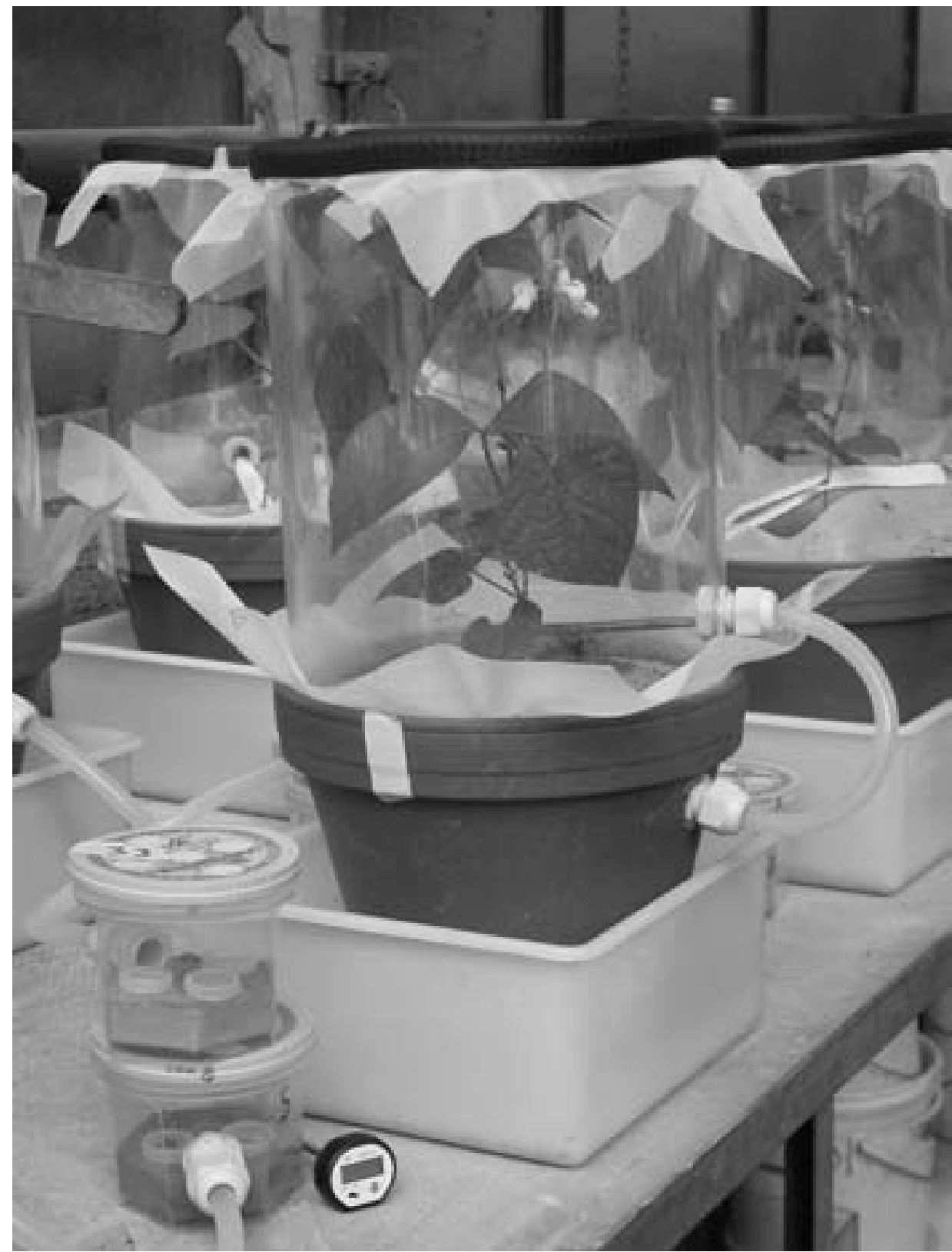

Abb. 35: Mikrokosmos mit Buschbohne, Blattläusen und Ameisenkolonie im Wurzelraum. Oberirdischer und unterirdischer Teil sind durch Gaze voneinander und von der Umgebung getrennt. Durch einen Schlauch (links im Bild) baben die Ameisen Zugang zum Spross mit den Blattläusen (über eine „Brücke“" erleichtert, weil der Wurzelhals mit Silikonfett in die Gaze eingepasst ist), durch einen zweiten (binten links) haben sie Zugang zu einer Futterkammer mit Aktivkoble/GipsGemisch und drei Futterschälchen (vorne links). 
Jeder Kosmos wurde zu Beginn mit $220 \mathrm{mg} \mathrm{KH}_{2} \mathrm{PO}_{4}$ gedüngt, um einen Phosphatgehalt von etwa $26 \mathrm{~kg} \mathrm{P} /$ ha zu erreichen und mit einer Suspension aus Wurzel-Knöllchen von zuvor angezogenen Buschbohnen geimpft, um die $\mathrm{N}_{2}$ Fixierung zu erhöhen. Jeweils die Hälfte von 8 Versuchseinheiten mit gleicher Fütterung wurde zusätzlich mit Stickstoff $\left(2 \times 80 \mathrm{mg} \mathrm{N}=2 \times 228,6 \mathrm{mg} \mathrm{NH}_{4} \mathrm{NO}_{3}\right.$ $=$ ca. $45 \mathrm{~kg} \mathrm{~N} / \mathrm{ha}$ ) gedüngt.

Vier Parallelen der acht Varianten wurden in Blöcken im Gewächshaus der Zoologie in Göttingen angezogen. Die Verteilung innerhalb der Blöcke geschah rein zufällig (Kartenziehen). Nach zwei Monaten wurden Spross, Wurzel, Früchte und die Blattläuse getrocknet und gewogen und die Ameisen und ihre z.T. verbliebenen Larven gezählt. Aus jedem Kosmos wurde Tier- und Pflanzenmaterial gefriergetrocknet und die Gehalte an Stickstoff und Kohlenstoff sowie die jeweiligen Isotopenverhältnisse analysiert. Eine genauere Versuchsbeschreibung wird bei Schumacher (2004) gegeben.

\subsection{Ergebnisse}

\subsubsection{Nahrungsnetzanalyse}

Einordnung der Ameisen in das Nahrungsnetz.

Die Pflanzen aus den drei funktionellen Gruppen unterschieden sich deutlich: Moose hatten mit -5 den niedrigsten $\delta^{15} \mathrm{~N}$-Wert, Kräuter mit $-3,5$ den höchsten; die Gräser lagen intermediär, unterschieden sich aber durch höhere ${ }^{13} \mathrm{C}$-Gehalte (Abb. 36. ; Sanders 2004, Sanders \& Platner, unveröffentlicht).

Die Pflanzensaftsauger hatten meist sehr ähnliche Werte wie ihre Nahrungsquelle. Die meisten analysierten Tierarten zeigten gegenüber der Basis eine Anreicherung des schwereren Stickstoff-Isotops ${ }^{15} \mathrm{~N}$, die bis zu $10 \%$ höheren DeltaWerten reichten. Durchschnittlich wurde das schwerere Kohlenstoff-Isotop ${ }^{13} \mathrm{C}$ dabei ebenfalls um etwa $1 \% 0 \delta^{13} \mathrm{C}$ pro $3 \% \delta^{15} \mathrm{~N}$ angereichert. Einige Saprophage hatten deutlich höhere $\delta^{13} \mathrm{C}$-Werte, die sich unter den Zoophagen z.B. bei Lasius flavus und Atypus wiederfinden. Einige Spinnen (wie Clubiona, Meioneta, Tibellus und juvenile Pisaura) haben kaum höhere $\delta^{15} \mathrm{~N}$-Werte als epigäische Collembola. Andere Spinnen, wie adulte Arctosa, Mangora oder Pardosa stehen mit $\delta^{15} \mathrm{~N}$-Werten von etwa $+4 \%$ an der Spitze des untersuchten Nahrungsnetzes. Mit $\delta^{15} \mathrm{~N}-$ Differenzen von etwa 4-8 \%o zu den Pflanzen gehören die Ameisen eindeutig zu den Prädatoren. Ponera coarctata und Formica cunicularia hatten etwa $2 \%$ höhere $\delta^{15} \mathrm{~N}$-Werte als die beiden Lasius-Arten. 


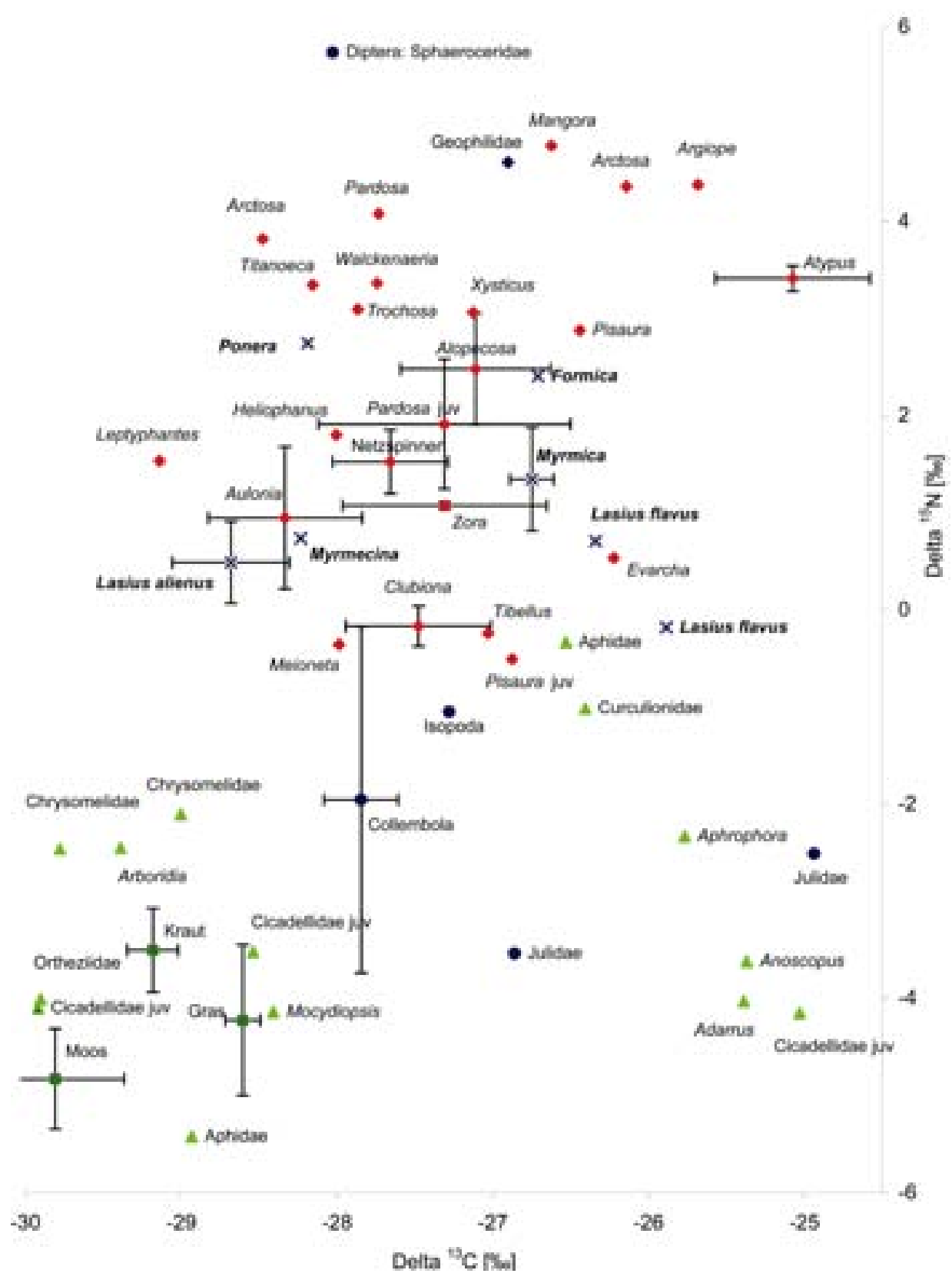

Abb. 36: Mittlere Gehalte der stabilen Isotope ${ }^{13} \mathrm{C}$ und ${ }^{15} \mathrm{~N}$ von Pflanzen (ロ), Ameisen (囚),

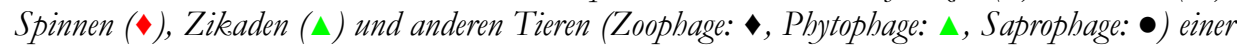
Brachwiese im September 2002, angegeben in Delta-Werten (relative Unterschiede zu Standards, vgl. Kap. 3.5). Bei mehr als vier analysierten Proben mit Standardabweichungen (Sanders, Rachor \& Platner, unveröffentlicht). 
Vergleich verschiedener Ameisenarten und ihrer Entwicklungsstadien

Schon die einzelnen Körperteile derselben Individuen unterscheiden sich deutlich in den $\delta^{13} \mathrm{C}$ - und $\delta^{15} \mathrm{~N}$-Werten. Der Gaster von Formica pratensis hat etwa 0,5\%o niedrigere $\delta^{15} \mathrm{~N}$-Werte als Kopf und Thorax. Dieser Unterschied war bei allen analysierten Individuen aus zwei verschiedenen Nestern ähnlich ausgeprägt (Abb. 37).

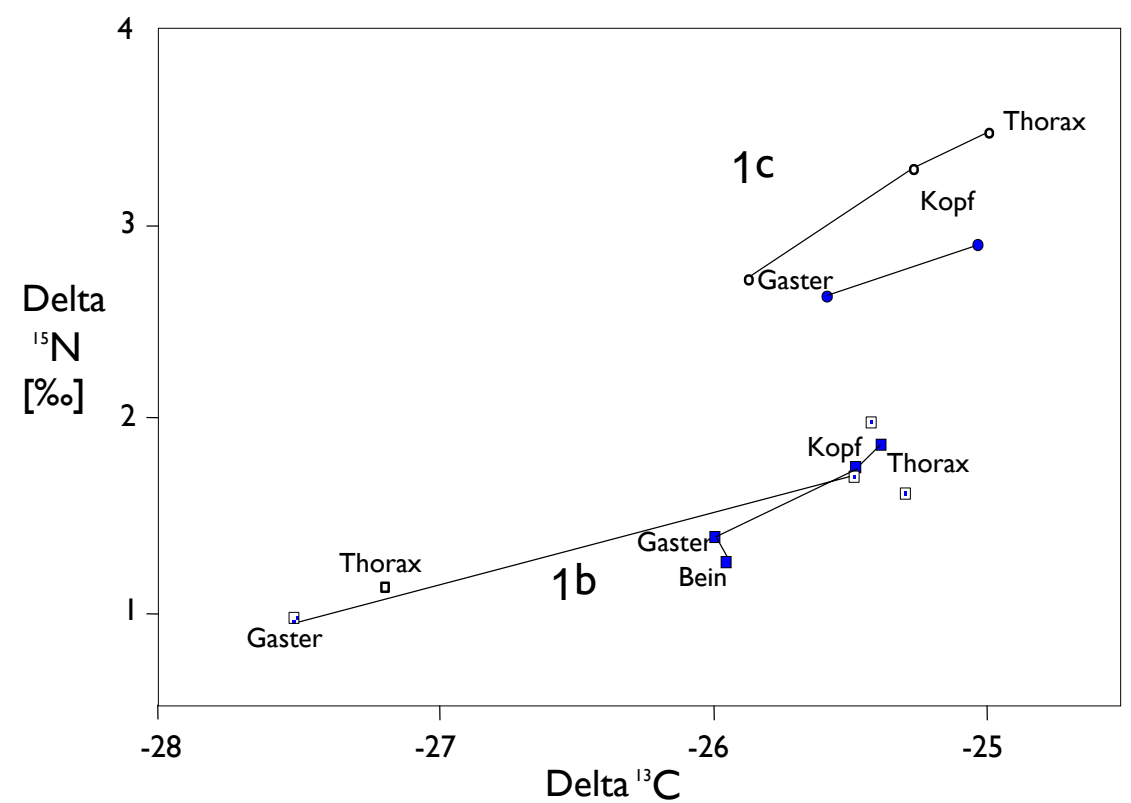

Abb. 37: Mittlere Gebalte der stabilen Isotope ${ }^{13} \mathrm{C}$ und ${ }^{15} \mathrm{~N}$ in verschiedenen Körperteilen von Arbeiterinnen der Ameise Formica pratensis in Delta-Werten (relative Unterschiede zu Standards, vgl. Kap. 3.5). Proben von denselben Individuen sind miteinander verbunden; Quadrate: Nesthügel auf Teilfläche 1b, Kreise: Nesthügel auf Teilfläche 1c, Juni 2000.

Auf Wiese 1 hatte das Gras $\delta^{15} \mathrm{~N}$-Werte von -4,9\%o und $\delta^{13} \mathrm{C}$-Werte von -29,6\%o, so dass die pflanzliche Basis des Nahrungsnetzes im Sommer 2000 vergleichbare Werten wie im Sommer 2002 hatte. Die Blattläuse hatten auch im Juni 2000 Werte ähnlich denen der Pflanzen $\left(\delta^{15} \mathrm{~N}:-4,1 \%\right.$ und $\left.\delta^{13} \mathrm{C}:-29,1 \% 0\right)$. Mit etwa 5\%o über der Basis liegenden $\delta^{15} \mathrm{~N}$-Werten hatte Lasius alienus auch im Sommer 2000 unter den Ameisen die niedrigsten Gehalte an ${ }^{15} \mathrm{~N}$. Die Arbeiterinnen aus der Lasius flavus-Kolonie hatten etwas höhere Gehalte des schwereren Stickstoff-Isotops, aber fast die gleichen $\delta^{13} \mathrm{C}$-Werte wie ihre Verwandten aus der gleichen Gattung. Die Arbeiterinnen der Gattungen Myrmica und Formica wiesen unter den Ameisen die größten Differenzen in den ${ }^{15} \mathrm{~N}-$ Gehalten zur Basis auf. Die maximalen Gehalte konnte ich bei den Sklavenjägern Polyergus rufescens und ihren versklavten Formica cunicularia Arbeiterinnen finden; die Arbeiterinnen der zweiten versklavten Art im Nest, Formica fusca, hatten deutlich niedrigere $\delta^{15} \mathrm{~N}$-Werte. 


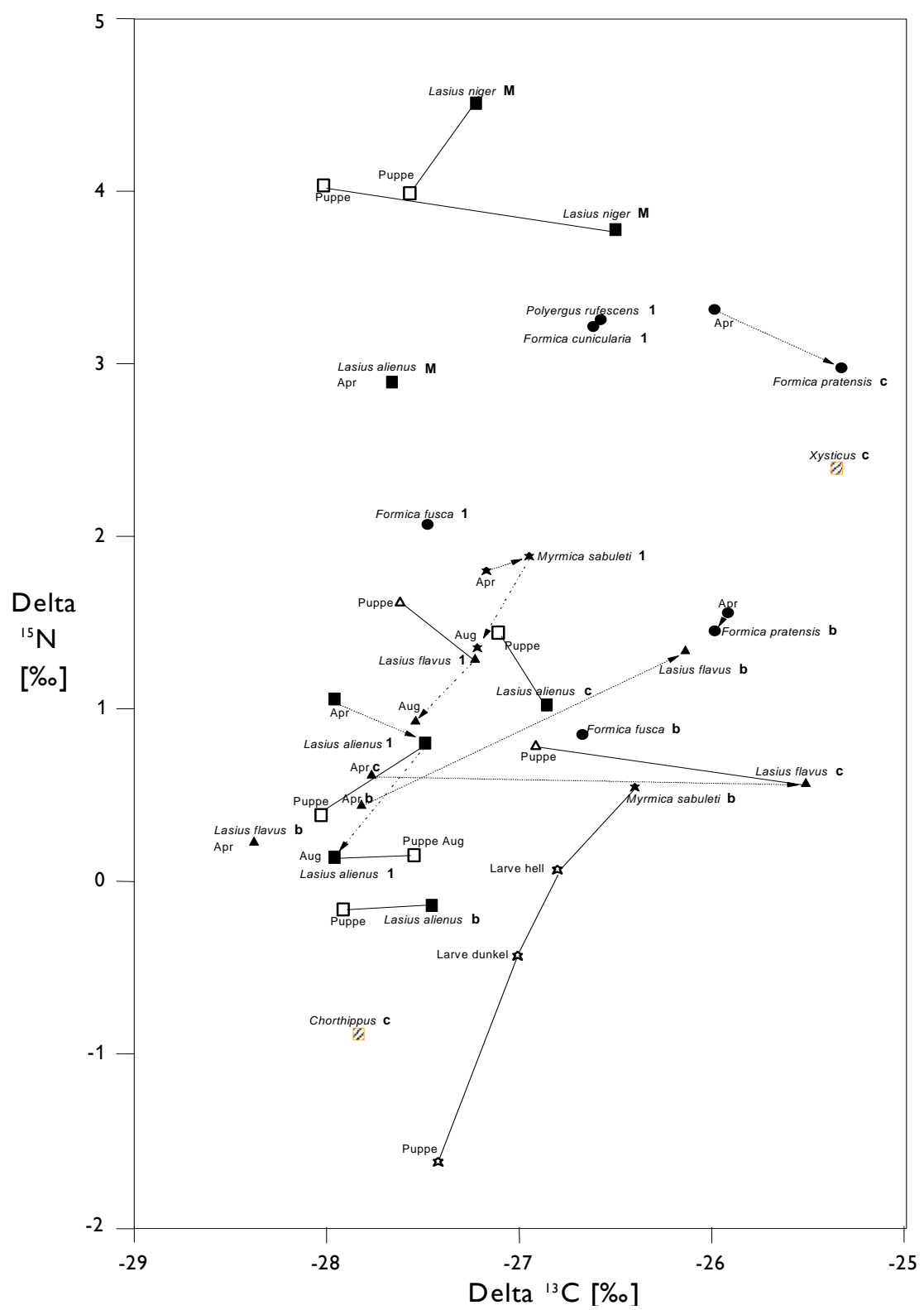

Abb. 38: Mittlere Gehalte der stabilen Isotope ${ }^{13} \mathrm{C}$ und ${ }^{15} \mathrm{~N}$ von Arbeiterinnen (ausgefüllte Symbole), Puppen und Larven (jeweils offene Symbole) der Ameisen Lasius alienus / L. niger (Quadrate), L. flavus (Dreiecke), Formica spec. (Kreise) und Myrmica sabuleti (Sterne) in DeltaWerten (relative Unterschiede zu Standards, vgl. Kap. 3.5). Proben aus denselben Kolonien sind miteinander verbunden, Pfeile markieren den zeitlichen Verlauf; Apr: April, Aug: August, obne Angabe: Juni 2000. $1=$ Kolonie auf Wiese1; $\boldsymbol{b}, \boldsymbol{c}=$ Kolonie auf Teilfläche $1 b$ brw. $1 c ; M=$ Kolonie auf „Maisfeld" (Grenze Mais-/Kleefeld). Zum Vergleich sind die Messwerte eines Phytophagen (Chorthippus) und eines Zoophagen (Xysticus) aufgetragen. 
Auf der benachbarten Streuobstwiese (1b) war das relative Muster der Arten zueinander sehr ähnlich, allerdings mit durchweg etwas geringeren $\delta^{15} \mathrm{~N}-$ Werten. Arbeiterinnen von Lasius flavus hatten hier von April bis Juni eine so hohe weitere Anreicherung, dass sie das Niveau der großen Formica pratensis erreichten.

Die beiden Lasius-Arten auf der Teilfläche 1c lagen auf einer trophischen Ebene genau zwischen der phytophagen Feldheuschrecke Chorthippus und der rein zoophagen Krabbenspinne Xysticus. Auch hier hat Lasius flavus von April bis Juni vor allem das Isotop ${ }^{13} \mathrm{C}$ in hohe Maße angereichert.

Die Lasius niger und L. alienus Kolonien am Rande des Maisackers hatten die größten Anteile an ${ }^{15} \mathrm{~N}$ aller untersuchten Tiere angereichert, zeigten jedoch keine Unterschiede in den ${ }^{13} \mathrm{C}-$ Gehalten zu den Ameisen von den anderen Flächen, nur gegenüber den Puppen der jeweils eigenen Kolonie hatten die Arbeiterinnen leicht erhöhte Anteile des schwereren Isotops ${ }^{13} \mathrm{C}$ in ihrer Körpersubstanz.

Für die Entwicklungsstadien lässt sich keine einheitliche Tendenz erkennen, die $\delta^{15} \mathrm{~N}-$ Werte der Puppen der Lasius-Arten unterscheiden sich im Mittel nur um etwa 0,2\% von denen der adulten Arbeiterinnen. Nur bei der Knotenameise Myrmica sabuleti von der Fläche 1b hatten die Puppen einen um $2 \%$ niedrigeren Wert als die Arbeiterinnen; die Larven lagen intermediär.

\subsubsection{Laborversuch}

Boden

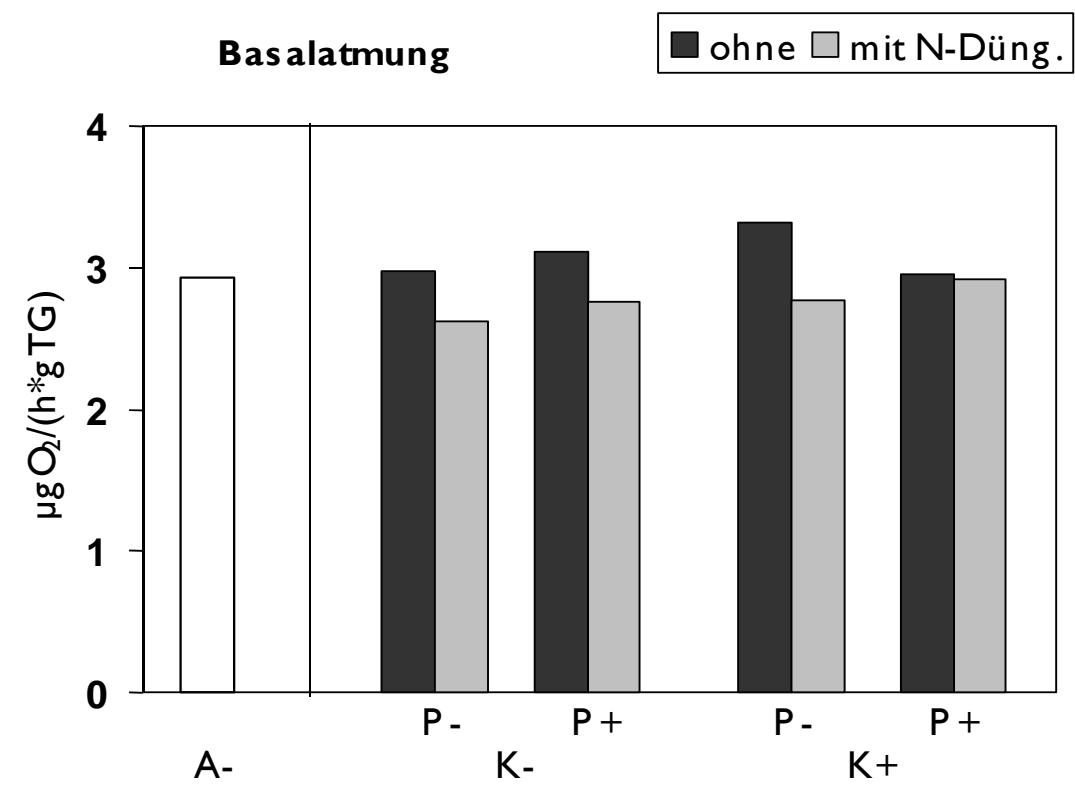

Abb. 39: Mittlere Basalatmung in Bodenproben ( $n=4)$ aus den Versuchsgefäßen nach Düngung bzw. zusätzlicher Fütterung $(K+, P+)$ der Ameisenkolonien. A-: Kontrollvariante obne Ameisen, K: Koblenhydrat (Robrzucker), P: Protein (Tenebrio-Larven); Schumacher \& Platner, unveröff. 
Weder Fütterung der Ameisen noch Düngung des Bodens veränderten die Wassergehalte des Bodens signifikant - allerdings führte die Anwesenheit von Ameisen zu einer leichten Erhöhung, wie der Vergleich zur Kontrolle ohne Ameisen zeigt $\left(24,3 \%, 25,5 \%\right.$ bzw. 27,4\% $\mathrm{H}_{2} \mathrm{O}$ vom Frischgewicht des Bodens in den ungedüngten Varianten ohne Ameisen, mit Ameisen bzw. mit Ameisen und Zufütterung). Zusätzliche Fütterung der Ameisen verstärkte diese Tendenz. Die Basalatmung wurde durch Düngung signifikant herabgesetzt, die mikrobielle Biomasse hingegen eher durch zusätzliche Proteinversorgung der Ameisen erhöht, wenn auch nicht signifikant, so dass die spezifische Respiration in den gedüngten Varianten mit Proteinfütterung der Ameisen deutlich geringer war.

\section{Buschbohnen}
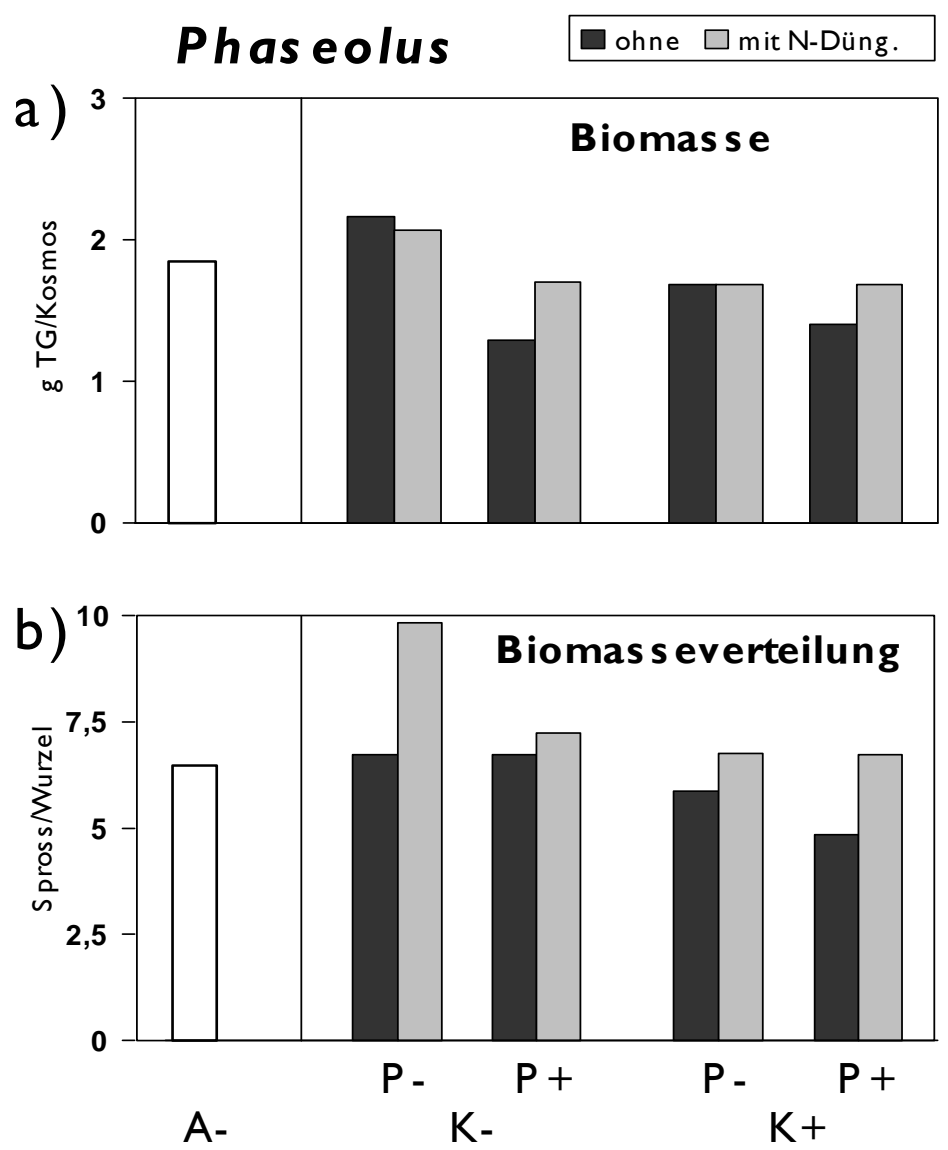

Abb. 40: Mittlere Pflanzen-Biomasse (a) und deren Verbältnis des oberirdischen Anteils zum unterirdischen Anteil (b) in den Versuchsgefäßen (n=4) nach Düngung bzw. zusätzlicher Fütterung $(\mathrm{K}+, \mathrm{P}+)$ der Ameisenkolonien. A-: Kontrollvariante obne Ameisen, K: Koblenhydrat Rohrzucker), P: Protein (Tenebrio-Larven); Schumacher \& Platner, unveröffentlicht. 
In den gedüngten Varianten bildeten die Buschbohnen im Durchschnitt weniger Wurzelknöllchen, ebenso in den Varianten mit zusätzlicher Proteinversorgung der Ameisen durch Mehlkäferlarven. Diese Unterschiede waren allerdings nicht signifikant $(\mathrm{p}<0,05$; F-Werte und Datengrundlage sind bei Schumacher (2004) angegeben). Ebenso veränderte sich weder die Biomasse der gesamten Pflanze noch die der Bohnen signifikant durch die unterschiedlichen Behandlungen. Die Trockengewichte der Sprosse unterschieden sich signifikant nur zwischen den Blöcken. Proteinzufütterung der Ameisen verringerte tendenziell die Phytobiomasse in den ungedüngten Varianten (Abb. 40 a); der Stickstoffgehalt des Sprosses wurde sowohl durch Düngung als auch durch Fütterung der Ameisen mit Mehlwürmern signifikant erhöht.

Das Verhältnis von ober- zu unterirdischer Biomasse wurde durch Düngung signifikant erhöht und durch zusätzliche Kohlenhydratversorgung der Ameisen signifikant verringert (Abb. 40 b, Tab. 16).

Tabelle 16: F-Werte und Wahrscheinlichkeiten für $\alpha$-Fehler (p) der Fütterungs-, Düngungs- und Blockeffekte und deren Interaktionen aus dreifaktoriellen Varianzanalysen der Ameisenaktivität und-abundanz, der Biomassen von Blattläusen und der Biomasseverteilung von Buschbohnen. FG: Freibeitsgrade (Effekt; Fehler); $p<0,1:$-Werte fett gesetzt; K: Koblenhydrat-Fütterung der Ameisen (Rohrzucker), P: Protein-Fütterung der Ameisen (Meblkäferlarven), N: Stickstoff-Düngung der Pflanzen.

\begin{tabular}{|c|c|c|c|c|c|c|c|c|c|}
\hline & \multirow[b]{2}{*}{$\mathrm{FG}$} & \multicolumn{2}{|c|}{$\begin{array}{c}\text { Lasius niger } \\
\text { Aktivität }\end{array}$} & \multicolumn{2}{|c|}{$\begin{array}{c}\text { Lasius niger } \\
\text { Individuen- } \\
\text { zahl }\end{array}$} & \multicolumn{2}{|c|}{$\begin{array}{l}\text { Aphis fabae } \\
\text { Biomasse }\end{array}$} & \multicolumn{2}{|c|}{$\begin{array}{c}\text { Phaseolus } v \text {. } \\
\text { Spross / } \\
\text { Wurzel }\end{array}$} \\
\hline & & $\bar{F}$ & $\bar{p}$ & $\bar{F}$ & $\bar{p}$ & $\bar{F}$ & $\bar{p}$ & $\bar{F}$ & $\bar{p}$ \\
\hline $\bar{K}$ & $1 ; 20$ & 24,60 & $<0,0001$ & 26,87 & $<0,0001$ & 0,01 & 0,9392 & 8,76 & 0,0075 \\
\hline P & $1 ; 20$ & 0,01 & 0,9421 & 0,19 & 0,6679 & 0,23 & 0,6459 & 3,54 & 0,0739 \\
\hline $\mathrm{N}$ & $1 ; 20$ & 0,11 & 0,7442 & 2,32 & 0,1424 & 4,69 & 0,0421 & 5,05 & 0,0356 \\
\hline $\mathrm{K} \times \mathrm{P}$ & $1 ; 20$ & 0,91 & 0,3504 & 0,00 & 0,9455 & 0,08 & 0,7831 & 0,06 & 0,8037 \\
\hline $\mathrm{K} \times \mathrm{N}$ & $1 ; 20$ & 0,11 & 0,7442 & 0,18 & 0,6719 & 0,41 & 0,5278 & 0,52 & 0,4791 \\
\hline $\mathrm{P} \times \mathrm{N}$ & $1 ; 20$ & 0,65 & 0,4280 & 2,66 & 0,1179 & 3,42 & 0,0786 & 1,02 & 0,3245 \\
\hline $\mathrm{K} \times \mathrm{P} \times \mathrm{N}$ & $1 ; 20$ & 0,13 & 0,7170 & 0,14 & 0,7146 & 0,68 & 0,4194 & 1,21 & 0,2829 \\
\hline Block & $3 ; 20$ & 1,38 & 0,2764 & 0,19 & 0,9054 & 3,30 & 0,0402 & 7,03 & 0,0019 \\
\hline
\end{tabular}




\section{Blattläuse}

Die zusätzliche Zuckerversorgung der Ameisen schien in der Anfangsphase negativ und später positiv auf die Blattläuse zu wirken, wie unsere Beobachtungen zeigten. Am Ende waren jedenfalls keine Unterschiede mehr feststellbar. Durch Düngung wurde die Gesamtbiomasse der Schwarzen Bohnenlaus signifikant erhöht. In den ungedüngten Varianten wirkte die zusätzliche Proteinquelle für die Ameisen positiv auf die Biomasse der Läuse; wenn zusätzlich gedüngt wurde, kehrte sich dieser Effekt um (signifikante Interaktion Dünger x Protein, Tab. 16). Die Blattläuse lagen in den Verhältnissen ihrer stabilen C- und N-Isotope sehr eng bei den Pflanzen. Der durch Düngung verringerte $\delta^{15} \mathrm{~N}$-Wert fand sich auch in den Blattläusen wieder.
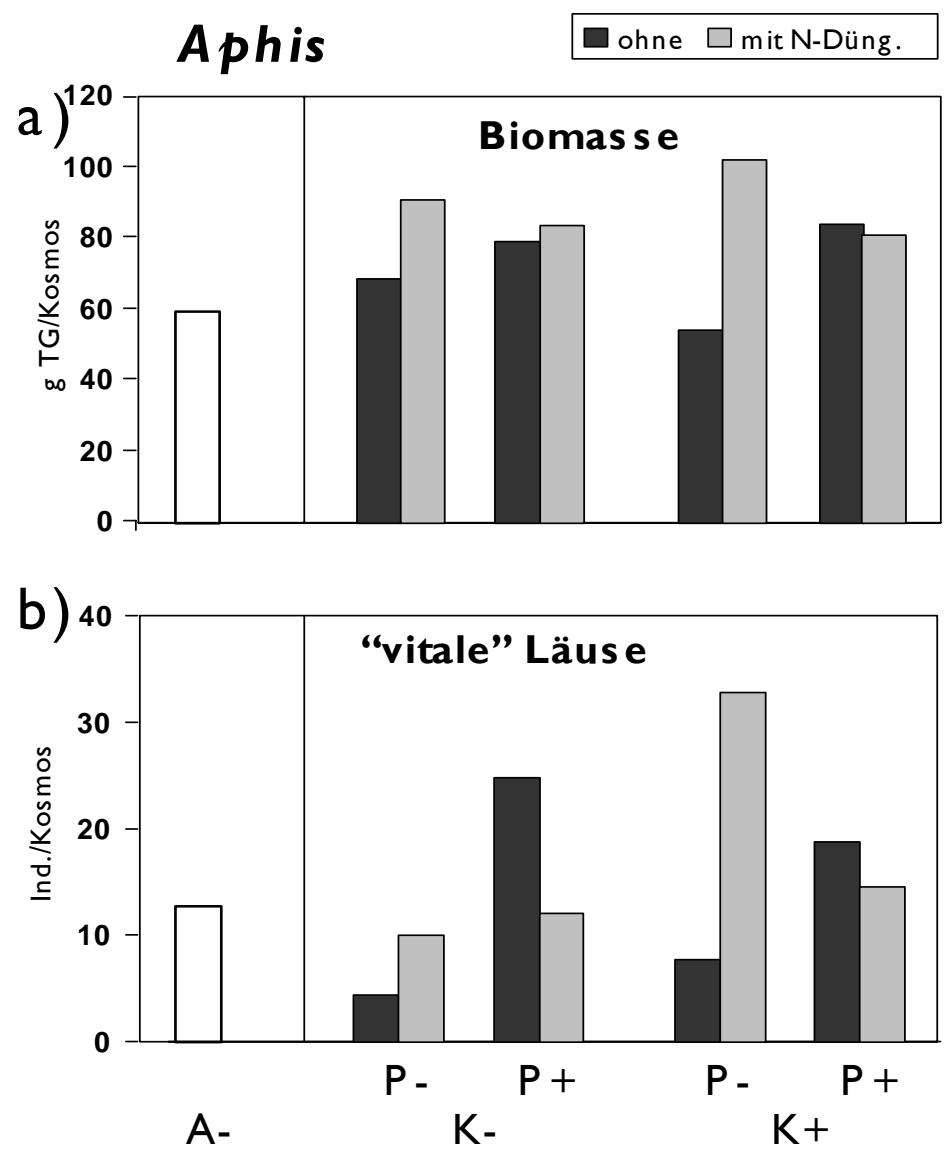

Abb. 41: Mittlere Blattlaus-Biomassen (a) und Anzabl „vitaler" Läuse (= lebende Läuse, die nach 48 h in einem Photoeklektor die abgeschnittene Pflanze verlassen hatten, $b$ ) in den Versuchsgefäßen nach Düngung bzw. zusätzlicher Fütterung der Ameisenkolonien. A-: Kontrollvariante obne Ameisen, K: Koblenhydrat (Robrzucker), P: Protein (Tenebrio-Larven), +: zusätəliches Angebot in der Futterkammer; $n=4$ je V ariante; Schumacher \& Platner, unveröffentlicht. 
Ameisen
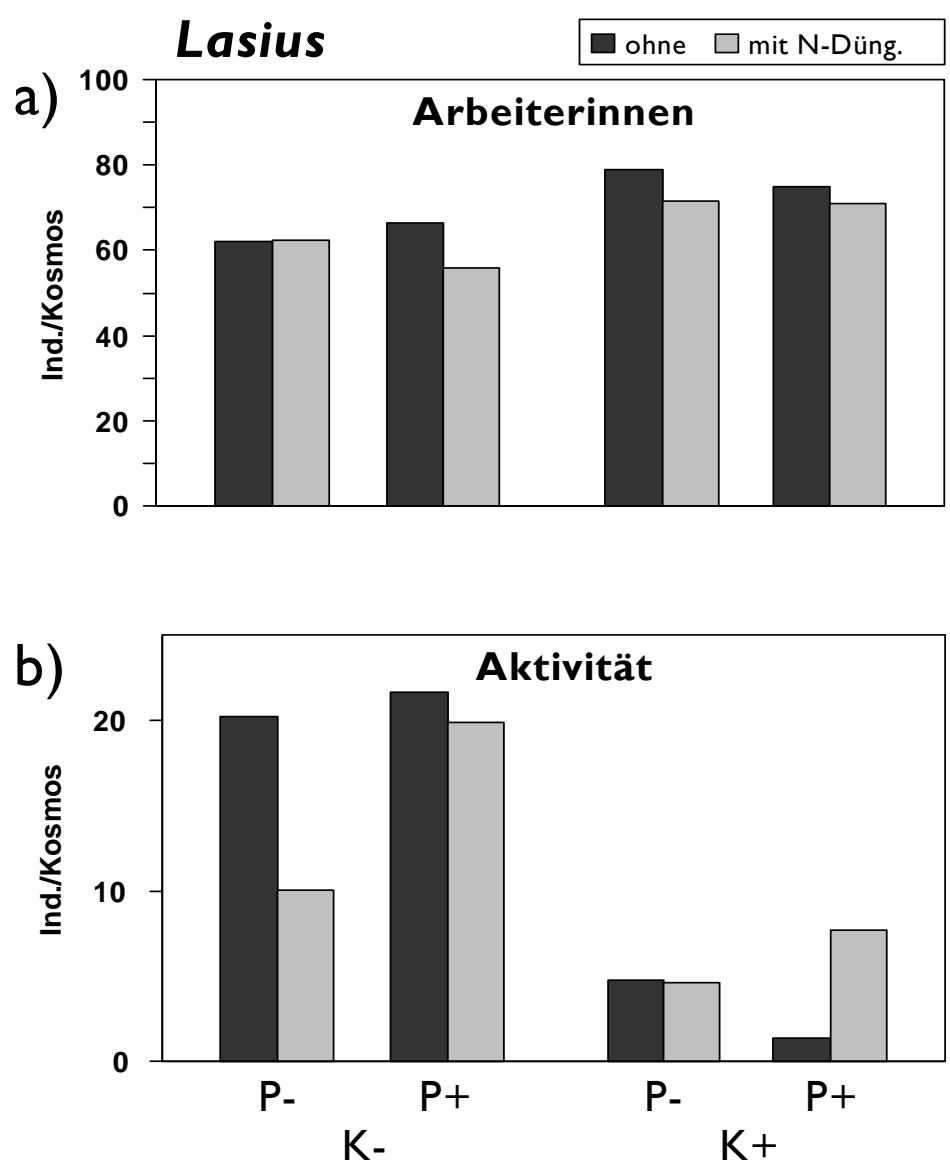

Abb. 42: Mittlere Anzabl lebender Arbeiterinnen zu Versuchsende (a) und deren Aktivität während des Versuchs (Anzabl beobachteter Arbeiterinnen, $\boldsymbol{b}$ ) in den Versuchsgefäßen nach Düngung bzw. zusätzlicher Fütterung $(K+, P+)$ der Ameisenkolonien. K: Koblenbydrat (Robrzucker), P: Protein (Tenebrio-Larven); $n=4$ je V ariante; Schumacher \& Platner, unveröffentlicht.

Zusätzliche Fütterung mit Rohrzuckerlösung wirkte positiv auf die Ameisen, am Versuchende waren in den Versuchsgefäßen mehr Arbeiterinnen als ohne zusätzliche Kohlenhydratquelle (Abb. 42 a, Tab. 16). Wenn in der Futterkammer Zuckerwasser angeboten wurde, ließ die Aktivität im oberirdischen Teil der Versuchsgefäße deutlich nach (Abb. 42 b). Die zusätzliche Fütterung mit TenebrioLarven wirkte sich hingegen nicht auf die Zahl der Arbeiterinnen aus. In den gleichzeitig gedüngten Varianten nahm die oberirdische Aktivität durch diese zusätzliche Proteinquelle zu. Düngung mit Ammoniumnitrat führte zu einer signifikant geringeren Larvenzahl mit gleichzeitig weniger überlebenden Arbeiterinnen. 
Beide Arten der Zufütterung der Ameisen wirkten sich auf die Gehalte der stabilen Stickstoff- und Kohlenstoffisotope aus. Die Gehalte der Arbeiterinnen an ${ }^{15 \mathrm{~N}}$ nahmen sowohl durch Düngung als auch durch Fütterung mit Rohrzuckerlösung signifikant ab, bei Angebot von Tenebrio erhöhten sie sich. Die $\delta^{13} \mathrm{C}$-Werte wurden durch die Nahrungsquellen auf C-4-Pflanzenbasis signifikant höher. Bei Fütterung mit Rohrzucker haben die Ameisen durchschnittlich etwa die Hälfte des Kohlenstoffs in ihrem Körper aus den zusätzlichen Futterquellen aufgebaut.

\section{Differenz zum Ausgangswert der Ameisen}

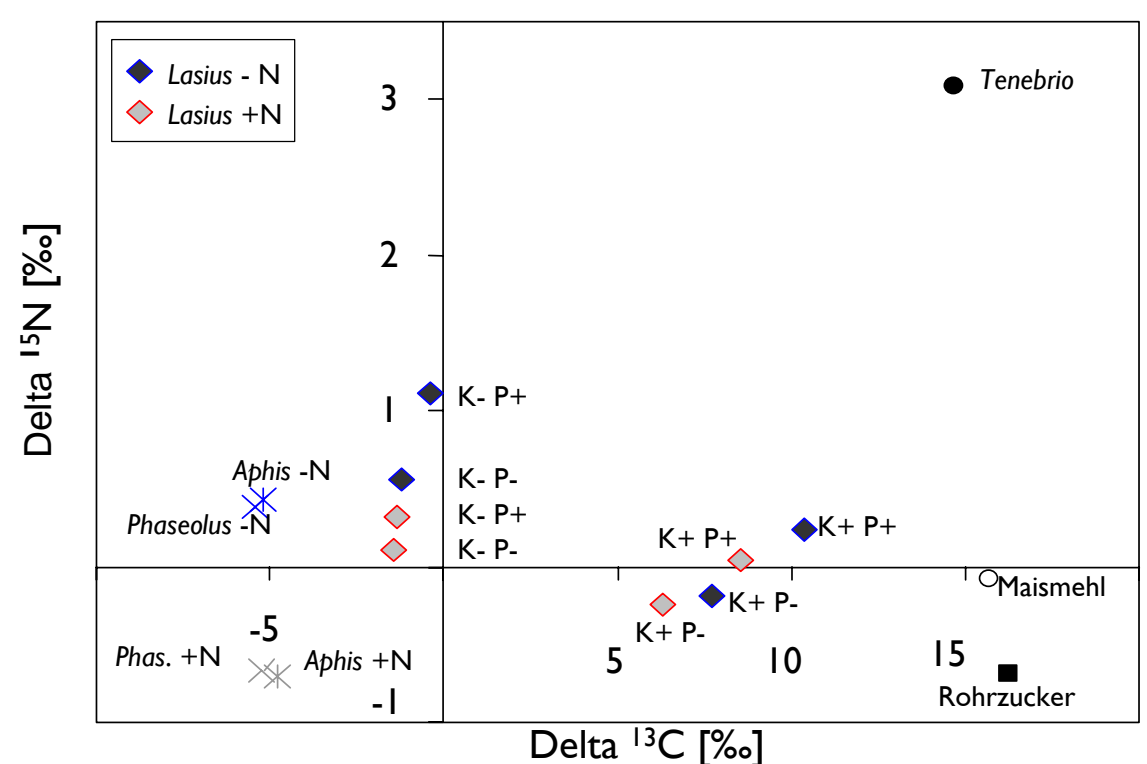

Abb. 43: Mittlere Verschiebung der Gehalte der stabilen Isotope ${ }^{13} \mathrm{C}$ und ${ }^{15} \mathrm{~N}$ von Arbeiterinnen der Ameise Lasius niger (Rauten) vom Wert ibrer Kolonie zu Beginn des Experients in den Fütterungsund Düngungsvarianten. Mittlere Differenz der Gehalte der stabilen Isotope ${ }^{13} \mathrm{C}$ und ${ }^{15} \mathrm{~N}$ von Phaseolus vulgaris (Kreuze), Aphis fabae (Sterne), Rohrzucker (Quadrat), Tenebrio melitor und Maismebl (Kreise) zu den mittleren Gehalten der Ausgangs-Ameisenkolonien ( $\delta^{13} \mathrm{C}:-27,01 \%$; $\delta^{15} \mathrm{~N}: 4,19 \%$ ) in Delta-Werten (relative Unterschiede zu Standards, vgl. Kap. 3.5). N: Amoniumnitrat ( ${ }^{15} \mathrm{~N}$-Differenz. zur Ausgangskolonie: -6,11\%o), K: Koblenhydrat (Rohrzucker), P: Protein (Tenebrio-Larven), +: zusätzliches Angebot in der Futterkammer bzw. Düngung mit N; $n=4$ je Variante; Schumacher \& Platner, unveröffentlicht.

\subsection{Diskussion}

Das Nahrungsnetz der Brachwiese ist nur unvollständig erfasst, da einige wichtige Taxa nicht auf Artniveau ausgewertet wurden. Dennoch erlaubt die vielfach replizierte Analyse der Ameisen und Spinnen eine detaillierte trophische Einordnung dieser wichtigen generalistischen Prädatoren. 
Es wird oft von einer Anreicherung des ${ }^{15} \mathrm{~N} /{ }^{14} \mathrm{~N}$-Verhältnisses von der Nahrung zum Konsumenten um 3 bis $4 \%$ ausgegangen (,trophic shift“; DeNiro \& Epstein 1978, Peterson \& Fry 1987). Doch es handelt sich um einen empirisch gefundenen Mittelwert; im Einzelfall kann die Anreicherung ganz andere Werte erreichen, je nach Tierart, Ernährungsstatus und Nahrung (Albers 2001, Vanderklift \& Ponsard 2003, Gaye-Siessegger et al. 2004). Auch die Anreicherung der Kohlenstoffisotope kann im Bereich einzelner Promille extrem variabel sein. Allerdings hat sich unter ,normalen“ Freilandbedingungen die Annahme von etwa 3 $\%$ Anreicherung des $\delta^{15} \mathrm{~N}$-Wertes pro trophischer Ebene als realistisch erwiesen (Scheu \& Falca 2000, Albers 2001, Oelbermann \& Scheu 2002). Im hier untersuchten Nahrungsnetz findet sich ein solcher Anreicherungswert beispielsweise zwischen Collembolen und juvenilen Netzspinnen, für die ein nachgewiesenes Räuber-Beute-Verhältnis besteht.

Die Ameisen hatten einen etwa 5 bis $8 \%$ höheren $\delta^{15} \mathrm{~N}$-Wert als die Basis des untersuchten Netzes. Die meisten juvenilen Spinnen lagen etwa 4 bis $7 \%$ über der Basis, die adulten bis zu $9 \%$. Man kann also etwa von drei trophischen Ebenen oberhalb der Basis der Produzenten ausgehen. Adulte Wolfsspinnen, große Netzspinnen, die großen Formica-Arten und die hypogäische, rein zoophage Ponera coarctata gehören zu den Spitzenräubern unter den Wirbellosen. Lasius, Myrmica, Myrmecina und die Jungspinnen wären Prädatoren der direkt an der Basis lebenden Phytophagen und Saprophagen und zum Teil gleichzeitig Beute der größeren Spinnen und Ameisen. Die etwa jeweils eine halbe trophische Ebene unter den Spinnen liegenden Werte der Ameisen wären sehr gut mit der teilweisen Versorgung aus Honigtau erklärbar. Für einige Arten liegen die $\delta^{13} \mathrm{C}$-Werte allerdings weit über der Basis und damit wesentlich höher als die durchschnittlich angenommen 1-2\%o Anreicherung.

Offenbar existieren für die Bodentiere ganz andere Nahrungskanäle; die Haupt-Ressource, Streu und Mikroflora, konnte hier nicht hinreichend untersucht werden, es gibt aber zahlreiche Hinweise, dass z.B. Pilze das schwerere Kohlenstoff-Isotop anreichern (Albers 2001). Innerhalb der Ameisen lassen sich einige Trends erkennen: Lasius flavus könnte stärker am Boden-Saprophagen-System hängen, Formica steht deutlich etwas höher als die anderen Gattungen, vermutlich überwältigt sie aufgrund ihrer Größe öfter andere Prädatoren. Große, adulte Spinnen stehen meist auch bis zu einer ganzen trophischen Ebene über den kleineren, juvenilen Spinnen, was die häufig beschriebene Intragilden-Prädation (Wise 1993) bestätigen könnte. Allerdings könnte auch einfach durch häufigere Metabolismuszyklen mit steigendem Lebensalter eine Anreicherung des $\delta^{15} \mathrm{~N}$-Wertes stattfinden (Oelbermann \& Scheu 2002).

Auch beim Vergleich der Ameisen untereinander bestätigt sich die etwas basalere Einordnung der Lasius-Arten und die der offensichtlich näher an der Spitze stehenden Formica-Arten. Zwischen Puppen und Arbeiterinnen gibt es keine deutlichen Unterschiede, bis auf die Ausnahme in einem Myrmica-Nest. Jedenfalls konnte eine möglicherweise höhere Trophie-Ebene der Larven aufgrund ihres erhöhten Protein-Bedarfs nicht bestätigt werden. Innerhalb eines Nestes sind die 
Schwankungen der Verhältnisse der stabilen Isotope in den meisten Fällen sehr niedrig, selbst Polyergus rufescens hat die gleichen $\delta^{13} \mathrm{C}$ - und $\delta^{15} \mathrm{~N}$-Werte wie eine ihrer Sklaven-Arten. Durch Trophallaxis ist ein ständiger Nahrungsaustausch zwischen den Nestgenossinnen möglich.

Der Kohlenstoff aus dem Mais geht wenig in die von den Ameisen genutzten Kohlenstoffflüsse ein, hingegen scheint der von den Symbionten der Leguminosen fixierte Stickstoff schnell Eingang in die Nahrungsressourcen von Lasius niger zu finden. Stickstoffreiche Pflanzen sind für Blattläuse besonders attraktiv (Nentwig et al. 2004), die Ameisen scheinen ihre Nahrung auch bevorzugt dem auf Klee basierendem Nahrungsnetz zu entnehmen.

Im Laborversuch konnte festgestellt werden, dass der von den Ameisen inkorporierte Kohlenstoff überwiegend aus zuckerhaltiger Nahrung stammt. Zusätzliche Fütterung mit Kohlenhydraten wirkte positiv auf die Ameisen, ihre oberirdische Aktivität bei der Blattlauskolonie verringerte sich. Etwa die Hälfte des körpereigenen Kohlenstoffs wurde in 2 Monaten aus dem Rohrzucker aufgebaut.

Die Fütterung mit Proteinen wirkte sich nicht so deutlich aus, hatte aber bei allen gemessenen abhängigen Größen eine Tendenz entsprechend den aufgestellten Hypothesen: eine positive Wirkung auf die Blattläuse und eine indirekte negative Wirkung auf die Pflanzen. Bei Kolonien mit Königin könnte diese Tendenz über einen längeren Zeitraum auch signifikante Effekte haben, da va. der Nachwuchs Proteine benötigt und erst bei einem ständigen hohen Bedarf die NährstoffBalance-Hypothese größere Bedeutung erlangen kann.

Entsprechend hatte im Versuch - ohne neu hinzukommende Larven - auch nur die Zuckerfütterung signifikante indirekte top-down-Effekte, die Pflanzen bildeten weniger oberirdische Biomasse im Verhältnis zu den Wurzeln aus. Die Biomasse der Läuse zeigte allerdings keine Unterschiede, so dass bei der Wirkung auf die Pflanzen evtl. auch direkt die ober- und unterirdische Aktivität der Ameisenkolonie eine Rolle gespielt haben kann. Bei Zufütterung von Zuckerwasser waren deutlich weniger Arbeiterinnen im oberen Teil der Mikrokosmen aktiv, so dass dort auch weniger Honigtau abgesammelt wurde, was in der zweiten Versuchshälfte zu einer stärkeren Verpilzung der Blattlauskolonien und damit auch der Laubblätter geführt hat; allerdings wurde die Verpilzung nur optisch geschätzt und nicht quantitativ gemessen.

Das zusätzliche Angebot an Zucker hat zu einer signifikant erhöhten Überlebensrate der Ameisen geführt, was insofern verwundert, als dass der Honigtau der Blattläuse vermutlich nicht zu knapp war, da von Beginn an eine starke Verpilzung auf der Gaze unterhalb der Lauskolonien zu beobachten war und Honigtau wegen seiner Zusammensetzung aus Aminosäuren und verschiedenen Kohlenhydraten für Ameisen attraktiver sein sollte als reines Zuckerwasser. Der Honigtau der fakultativ myrmecophilen Art Aphis fabae ist allerdings quantitativ und qualitativ dem anderer Arten unterlegen, da er in vergleichsweise geringen Mengen anfällt und keine für Ameisen hochattraktiven Trisaccharide enthält (Völkl et al. 1999).

Zucker stellt für Ameisen die Hauptenergiequelle dar (Carroll \& Janzen 1973), die letztlich auch für den Beutefang benötigt wird, weshalb eine bessere Versor- 
gung mit Honigtau oft zu einer verstärkten Prädation führt, die sowohl andere Blattläuse als auch die Honigtaulieferanten selbst betreffen kann (Sakata 1995, 1999, Fischer et al. 2001, Offenberg 2001). Zum einen bedeutet das Konkurrenz um die Betreuung durch Ameisen (Völkl et al. 1999, Fischer et al. 2001) zum anderen sind für die betreuten Blattläuse eindeutige Kosten auf individueller sowie Populations-Ebene damit verbunden, die sogar die Vorteile des Schutzes vor anderen Prädatoren ausgleichen können (Stadler 2001). Durch zusätzliches Angebot von Zucker in der Futterkammer wurden auch in unserem Experiment die ebenfalls dort angebotenen Mehlwürmer verstärkt eingetragen.

Das angeborene Verhalten der Ameisen führt primär zu einer Sicherstellung der Energieversorgung der Kolonie und erlaubt es erst dann einer größeren Zahl an Arbeiterinnen, gezielt Jagd auf Beutetiere zur Versorgung der Larven zu machen. Die individuellen Verhaltensweisen hängen letztlich von der Bereitschaft ab, eine Instinkthandlung auszuführen, wodurch das Verhalten der ganzen Kolonie flexibel auf die (durch Stärke bestimmter Schlüsselreize vermittelten) Bedürfnisse reagieren kann (Otto 2003). Die entwicklungsabhängigen individuellen Tätigkeitsprofile der einzelnen Arbeiterinnen waren wohl auch der Grund dafür, dass die aus Nestern entnommenen Arbeiterinnen in den Versuchsgefäßen oft erst nach einigen Tagen die Futterquellen und die Wege dorthin erkundeten und entsprechend nutzten. Dadurch hatten die Blattläuse etwas mehr Zeit, sich im feindfreien Raum ungestört zu vermehren, was eine spätere signifikante Kontrolle der Population durch die 50 Arbeiterinnen erschwert haben könnte. Insgesamt haben sich die Versuchsgefäße aber bewährt und bieten attraktive Möglichkeiten der weiteren Untersuchung der ober- und unterirdischen Wirkungen von Ameisen.

Stickstoffdüngung wirkt positiv auf Pflanzen und Läuse, die Buschbohne bildet weniger Wurzelknöllchen, was sich auch deutlich auf den ${ }^{15} \mathrm{~N}-$ Gehalt auswirkt. Dieser Effekt zeigt seine Wirkung bis in die dritte trophische Ebene bei den Ameisen. Die Mikroflora scheint effektiver zu werden, da weniger geatmet werden muss, um die gleiche Biomasse aufbauen zu können. Allerdings war keine Steigerung der Produktivität der Buschbohne durch Düngung festzustellen. Die Biomasse der Blattläuse wurde bei Düngung nicht durch den vermuteten stärkeren Prädationsdruck der Ameisenkolonien ohne Protein-Zufütterung vermindert, so wie es ohne Düngung der Fall war. Die positive Wirkung der Düngung auf die Läuse könnte also eine evtl. positive Wirkung auf die Pflanze durch negative Rückkoppelung wieder ausgeglichen haben.

Auch die Wirkung der Ameisen im Nahrungsnetz hängt stark von der Ressourcenverfügbarkeit ab; letztlich unterliegen auch die top-down-Wirkungen generalistischer Prädatoren einer bottom-up-Kontolle, so dass für eine Synthese unbedingt beide Wirkungsrichtungen gemeinsam betrachtet werden müssen (Walker \& Jones 2001). Neben der zentralen Abhängigkeit von der Sonneneinstrahlung (Kap. 5) besteht für Ameisen eine weitere in Form ausreichender Verfügbarkeit von verwertbaren Kohlenhydraten. Sind diese beide Hauptbedingungen erfüllt, können Ameisen eine zentrale Stellung im Nahrungsnetz einnehmen. 


\section{Interaktionen mit Spinnen und Phytophagen auf Halbtrockenrasen: Intragilden-Prädation, Konkur- renz und Trophobiose}

\subsection{Einleitung}

Ameisen können als soziale Insekten und durch die Versorgung mit Honigtau lokal sehr hohe Populationsdichten erreichen und konstant erhalten, was sie, in Verbindung mit ihrer hohen Aktivität auf den Wirts-Pflanzen der TrophobiosePartner, dazu befähigt, andererseits viele Fraßfeinde der Pflanzen zu kontrollieren (z.B. Messina 1981, Bristow 1984).

Gerade bei Formica-Waldameisen ist eine mögliche indirekt positive Wirkung der Ameisen und damit auch der Trophobiose auf die Pflanzen intensiv erforscht (Whittaker 1991, Way \& Khoo 1992). Die "grünen Inseln", die gelegentlich bei Schädlingskalamitäten rings um Waldameisennester entstehen, können mindestens teilweise auf diese top-down-Wirkung der Ameisen zurückgeführt werden (Laine \& Niemelä 1980, Karhu \& Neuvonen 1998, Karhu 1998).

Neben den Ameisen sind in vielen Lebensräumen Spinnen eine der wichtigsten Zoophagen-Gruppen (Wise 1993). In einem Graslandlebensraum in den Nordkarpaten erbeuteten nach einer Berechnung von Kajak et al. (1972) Ameisen der Gattung Myrmica mehr als $40 \%$ der Zikaden, mehr als $30 \%$ der Diptera und etwa $49 \%$ der vaganten Spinnen, die im gleichen Jahr produziert wurden. Obwohl die Ameisen meist eine etwas geringere Biomasse als die Spinnen hatten, war ihre 
Konsumption deutlich größer, sie können dabei auf einer naturnahen Wiese bis zum 200-fachen ihrer Biomasse an Beute konsumieren (Kajak et al. 1971); das Maximum der Prädation durch Ameisen lag im Frühsommer zur Zeit der maximalen Produktion und damit deutlich vor dem der Spinnen.

Die meisten korrelativen Beobachtungen und experimentellen Nachweise zur Struktur der Interaktionen von Ameisen und anderen dominanten Prädatoren stammen allerdings aus anderen Klimazonen oder Waldlebensräumen. Innerhalb einer Super-Kolonie von Formica lugubris fanden Cherix \& Bourne (1980) in Bodenfallen deutlich niedrigere Zahlen an anderen Ameisenarten und vaganten Wolfsspinnen (Lycosidae) als außerhalb. Brüning (1991) konnte jedoch keine signifikanten Unterschiede in der Spinnenfauna, die sich allerdings vorwiegend aus netzbauenden Arten zusammensetzte, innerhalb und außerhalb des Einflussbereiches einer Formica polyctena-Kolonie finden; in beiden Untersuchungen machten Spinnen weniger als $5 \%$ der Ameisen-Beute aus. In den Nesthügeln und in Mesokosmen direkt auf den Ameisenstraßen von Formica aquilonia war die Biomasse von epigäischen Raubarthropoden, in erster Linie Spinnen, deutlich niedriger als in der umgebenden Streu, bzw. in Mesokosmen abseits der Ameisenstraßen (Laakso \& Setälä 1998, Laakso 1999).

In einfachen Systemen konnte oftmals ein deutlicher Einfluss der generalistischen Spinnen auf phytophage Insekten nachgewiesen werden (z.B. Riechert \& Bishop 1990, Finke \& Denno 2003), doch in komplexen, naturnahen Systemen wie Halbtrockenrasen sind die Einflüsse generalistischer Prädatoren bisher weitgehend ungeklärt.

In einem faktoriell angelegten Ausschlussversuch wurden zwei dominierende Prädator-Gruppen des Halbtrockenrasens, Ameisen [v.a. Myrmica spec.] und Spinnen [in erster Linie Lycosidae als dominante vagante Jäger], in ihrer Wirkung aufeinander und auf die restliche Fauna untersucht. Durch selektive Dichtereduktion bzw. -erhöhung der beiden Taxa konnten

- ihre Einflüsse einzeln und in Interaktion auf die übrige Arthropodengemeinschaft und

- ihre Wechselwirkungen untereinander, wie z.B. die Rolle von ,intraguild predation"،

untersucht werden.

\subsection{Methodik}

\section{Versuchsaufbau}

Im Brachwiesenbereich von Wiese 1 und seinem Übergangsbereich zum Halbtrockenrasen wurden insgesamt 20 Versuchsparzellen eingerichtet. Dazu wurden mit Kunststoffzäunen (5-10 cm tief eingegrabene PE-Bahnen zu Kreisen geschlossen, ca. 25-30 cm Höhe, Verhinderung des Überkrabbelns durch einen Streifen Silikonfett und Kürzen der berührenden Vegetation) zwanzig je $2 \mathrm{~m}^{2}$ große Flächen im Freiland abgegrenzt. Oberflächenaktive Ameisen und vagante Spinnen (vorwie- 
gend Lycosidae) wurden selektiv von diesen Flächen ausgeschlossen. Die beiden Faktoren wurden in jeweils zwei Stufen (leicht erhöhte und deutlich reduzierte Dichte) etabliert, die sich ergebenden vier Varianten (Ameisendichte reduziert, Spinnendichte reduziert, die Dichten beider Gruppen reduziert und beide Gruppen in leicht erhöhter Dichte) wurden in je fünf Parallelen eingerichtet. Der Einfluss des Zauns wurde durch Vergleich mit Proben aus nicht abgegrenzten Bereichen abgeschätzt.

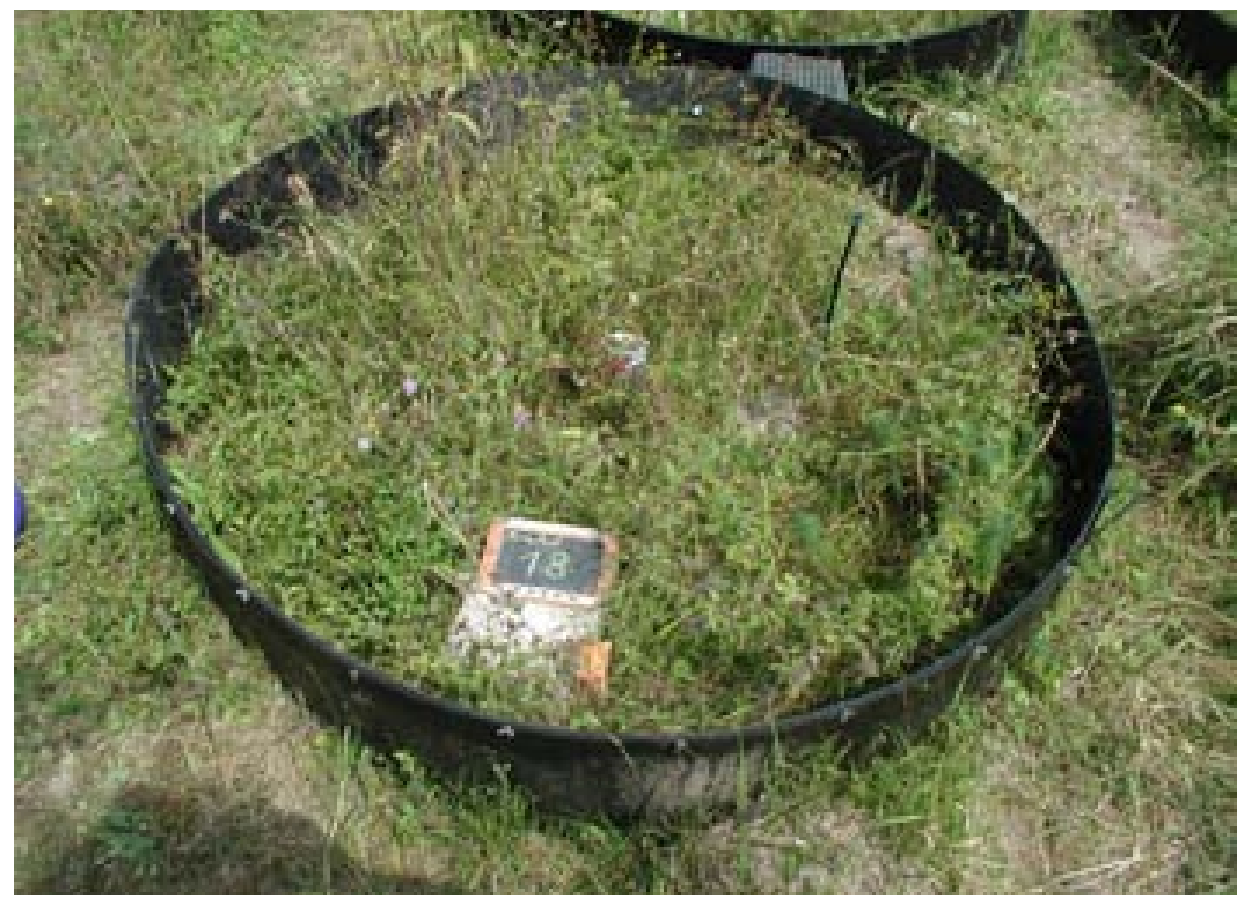

Abb. 44: Parzelle 18 des Ausscblussversuchs (bobe Ameisendichte, reduzierte Spinnendicbte). Im Zentrum stebt auf einer verschlossenen Lebendbodenfalle ein Datalogger für Temperatur- und Luftfeucbtigkeitsmessungen.

Auf Wiese 1 wurde der mittlere Brachwiesen-Bereich in fünf Hangabschnitte mit relativ einheitlicher Vegetation unterteilt, die einen Gradienten vom Halbtrockenrasen bis zur trockenen Glatthaferwiese abdeckten (vgl. Tab. 1, Kap. 2.4). Diese Abschnitte wurden in der Versuchsanlage statistisch als Blöcke behandelt. Auf jedem dieser Blöcke wurden mit Hilfe von Köderschalen mit Honigwasser (Abb. 20, S. 52) je zwei Bereiche mit Ameisennestern und hoher Arbeiterinnenaktivität an der Oberfläche festgestellt, in denen die zwei Parzellen mit hoher Ameisendichte eingerichtet wurden. Zwei weitere Parzellen wurden in Bereichen mit niedriger Ameisendichte eingerichtet. Jeweils einer der Parzellen mit hoher bzw. niedriger Ameisenaktivität wurde zufällig eine von zwei Behandlungsstufen des Faktors „Spinne“ zugeordnet, so dass jeder Block aus vier Parzellen mit den vier Behandlungsvarianten bestand. 
Durch ein Monitoring mit den Köderschalen wurde die Ameisenaktivität weiterhin überprüft und es wurden so viele Nester wie möglich ausfindig gemacht. Wurde ein Nest in Parzellen mit der Behandlungsstufe niedriger Ameisendichte gefunden, wurde es mit einem Bodenbohrer ausgestochen und gegen einen Bohrkern aus einer Parzelle mit hoher Aktivität im gleichen Block getauscht.

Die Umgrenzungen von Parzellen mit zugeloster Behandlung ,niedrige Spinnendichte" wurden außen mit Silikonfett eingeschmiert, um Einwanderung einzuschränken und es Spinnen zu ermöglichen, die Parzelle durch einen Sprung oder Abseilen von der Oberkante zu verlassen. Die übrigen Parzellen wurden innen eingeschmiert, um andersherum eine zusätzliche Einwanderung zu ermöglichen. Der Streifen Silikonfett war wetterbeständig und hinderte sowohl Spinnen als auch Ameisen bei normaler Aktivität daran, die Umgrenzung zu überklettern. In Fluchtsituationen konnten aber zumindest größere Spinnen den Fettstreifen problemlos überwinden, was einen Falleneffekt verhinderte. Die offenen Parzellen ermöglichten zudem eine ständige Wiederbesiedlung durch Aeronauten. An regelmäßigen Terminen in jeder Woche während der gesamten Versuchsdauer vom 30.04.2002 bis zu 09.09.2002 wurden deshalb alle Spinnen, die in einem jeweils gleichlangen Zeitintervall von Hand oder mit Hilfe eines Exhaustors in einer Parzelle gefangen werden konnten, abgesammelt und nach Bestimmung auf Gattung oder Familie in Parzellen mit hoher Spinnendichte innerhalb des gleichen Blocks eingesetzt. Am 26. Juni und 6. August wurden im Frühtau alle Spinnennetze gezählt und in den entsprechenden Parzellen entfernt.

Lebendbodenfallen in der Mitte jeder Parzelle ohne Fangflüssigkeit und mit einigen Laubblättern als Versteckmöglichkeit vor mitgefangenen Prädatoren erwiesen sich als ungeeignet zur Dichtereduktion von Spinnen, da innerhalb eines Öffnungs-Zeitraums von ein bis zwei Tagen nur einzelne Individuen erfasst und gegebenenfalls umgesetzt werden konnten.

Im August wurden sechs zusätzliche $1 \mathrm{~m}^{2}$ große Parzellen in den Blöcken 1, 3 und 5 eingerichtet, mit einem Sauger komplett abgesaugt und die Spinnen selektiv in je eine Parzelle auf jedem Block zurückgesetzt. Anschließend wurden die Parzellen mit engmaschiger Metallgaze verschlossen.

\section{Beprobung}

Neben dem regelmäßigen Monitoring der Spinnen und Ameisen wurde die Makrofauna an drei Terminen möglichst komplett mit Saug- und Bodenproben erfasst. Am 5. Juni wurde der $21 \mathrm{~cm} \varnothing$ Bodenbohrer einmal in jeder Parzelle und in jedem Block außerhalb der Umgrenzungen aufgesetzt und mit dem umgebauten Sauger (Kap. 3.1) ausgesaugt. Anschließend wurde der Bohrer eingeschlagen und die übrigen Tiere aus der Bodensäule durch Hitzeextraktion ausgetrieben. Dies ermöglichte einen direkten Vergleich der Effizienz jeder Methode und ließ durch Aufsummieren für die meisten Tiergruppen auch den Vergleich mit nichtabgesaugten Bodenproben zu. Der Flächenbezug der Saugmethode wird durch den Bohrzylinder wesentlich exakter, da ein Seitensog keine Rolle mehr spielt. Die Bohrlöcher wurden mit Holzbrettchen abgedeckt, was Verhaltensbeobachtungen 
an sich sonnenden Wolfsspinnen und das gezielte Absammeln sich tagsüber unter dem Brettchen versteckt haltender Spinnen (v.a. Clubionidae, Gnaphosidae, Aulonia) zuließ.

Am 15. August und 9. September wurde in jeder Parzelle und jedem Block eine Mischprobe durch dreimaliges Aufsetzen und Aussaugen des Bohrers genommen. Am 9. September wurde jeweils noch eine zuvor nicht abgesaugte KempsonProbe genommen, die am 4. September im Zentrum jeder Parzelle angebrachte Bodenfalle mit gesättigter Kochsalzlösung entnommen und die sechs zusätzlichen Käfige durch je zwei gleichartige Saug-Mischproben und einen Bohrereinstich mit anschließender Hitzeaustreibung beprobt.

\section{Auswertung}

Da die effektive Dichte der Ameisen in den Parzellen von einzelnen Proben nur ungenügend beschrieben werden konnte - die Saugfänge erfassten nur etwa 2 - 12 $\%$ der Arbeiterinnen, die Kempsonproben waren durch teilweise Nestanstiche extrem inhomogen (Sanders 2004) - habe ich alle Nachweise für epigäisch aktive Arten zusammengefasst. Dabei wurden alle Individuen, die in den einzelnen Parzellen durch Saug- und Bodenproben, beim regelmäßigen Monitoring mit Köderschalen und an den Brettchen und in den Bodenfallen gezählt wurden, aufaddiert; Nestnachweise wurden vorsichtig mit nur 100 Individuen (entsprechend der mittleren Zahl der in den Bodenproben nach Nestanstich gefundenen Arbeiterinnen) veranschlagt, umgesetzte Nester wurden entsprechend dem Datum der Umsetzung (Anteil des Zeitraums bis zur Umsetzung am gesamten Versuchszeitraum) teilweise der Quellparzelle und teilweise der Zielparzelle zugeordnet. Die verschiedenen Nachweis-Methoden sind zwar teilweise nur schlecht direkt quantitativ vergleichbar, ergeben aber durch die Gleichbehandlung aller Parzellen in der Summe ein brauchbares relatives Maß. Für jede Art wurde die Biomasse aus der Individuenzahl durch Multiplikation mit der mittleren Trockenmasse berechnet, die beim Einwiegen für die Analyse der stabilen Isotope bestimmt wurde.

Die Individuenzahlen aus den drei Saugfängen und den beiden Bodenproben wurden mit einer Varianzanalyse für Messwiederholungen (repeated measurement ANOVA) statistisch für die Effekte der Faktoren Spinne, Ameise, Block und die Interaktion von Spinne und Ameise analysiert, die aufsummierten Biomassen der einzelnen Arten mit einer multivariaten ANOVA und die Summe der epi- bzw. hypogäischen Ameisen mit einer univariaten ANOVA.

\section{Ameisenausschluss auf Bäumen und Sträuchern}

Am 20. Juni 2002 wurden auf den benachbarten Flächen 1a und 1b an Waldkiefern (Pinus sylvestris), Süßkirschen (Prunus avium) und Rosen (Rosa canina agg.) jeweils 12 etwa gleichgroße Zweige ausgewählt, von denen jeweils 6 mit grünen Raupenleimringen (Neudorf) belegt wurden, um den Zugang für Ameisen zu verhindern. Nach 2 Monaten, am 22. August, wurden alle 36 Äste mit Klopfschirm und Sauger in Kombination beprobt und die Tiere tiefgefroren. 


\subsection{Ergebnisse}

\section{Ameisen}

Die Biomasse der epi- und hypergäischen Ameisen war im Experiment in den Varianten mit reduzierter Ameisendichte signifikant verringert. Der größte Teil der Biomasse wurde von der sich hauptsächlich zoophag ernährenden Knotenameise Myrmica sabuleti ausgemacht, deren Dichte insgesamt signifikant erhöht bzw. reduziert werden konnte. An den einzelnen Probenahmeterminen waren die Unterschiede nicht signifikant, da die Zahl der Arbeiterinnen, die in jeder Parzelle erfasst werden konnten, zu stark schwankte. Meist waren in den Proben mit geringer Myrmica-Individuenzahl mehr Tiere der nächst häufigeren Art Lasius alienus vertreten. Lasius alienus war zu Beginn des Experiments in den Parzellen mit reduzierter Spinnendichte häufiger, hatte aber im Laufe des Sommers auf den Flächen mit höherer Spinnendichte und reduzierter Ameisendichte ihr Dichtemaximum in der Summe war auch diese Art in der Variante mit erhöhter Ameisendichte, in der die Spinnen abgesammelt wurden, am häufigsten.

Die großen Formica-Arten nisteten nur in einer Parzelle (Formica cunicularia in Parzelle 3), konnten aber vereinzelt auch in den anderen regelmäßig beobachtet werden, da sie offensichtlich deutlich leichter den Silikonfett-Streifen überwinden konnten. Im August war ihre Aktivität durch die experimentell erhöhte Spinnendichte signifikant negativ beeinflusst; in der Variante mit gleichzeitig reduzierter Ameisendichte waren auch in der Summe über die Termine und Methoden fast keine Arbeiterinnen aktiv (Abb. 45, Tab. 17). Die hypogäischen Arten Lasius flavus und Ponera coarctata wurden bei der Nestersuche nicht berücksichtigt und ihre Dichte entsprechend auch nicht manipuliert.

Tabelle 17: F-Werte und Wabrscheinlichkeiten für $\alpha$-Febler (p) der Behandlungs- und Blockeffekte und deren Interaktionen aus zweifaktoriellen Varianzanalysen der Trockengewichte von epigäischen Ameisen auf den Versuchsparzellen wäbrend des gesamten Zeitraums, von vaganten Spinnen im Juni-Saugfang und von netzbauenden Spinnen im September-Saugfang. FG: Freibeitsgrade (Effekt; Febler); $p<0,05:$ F-Werte fett gesetzt; $p>0,3$ nicht angegeben; log: Werte logtransformiert $\left(\log _{10} X+1\right)$. Spinnendaten aus Sanders (2004).

\begin{tabular}{|c|c|c|c|c|c|c|c|}
\hline & \multirow[b]{2}{*}{$\mathrm{FG}$} & \multicolumn{2}{|c|}{ Ameisen $^{\log }$} & \multicolumn{2}{|c|}{$\begin{array}{l}\text { vagante } \\
\text { Spinnen } \\
\text { (Juni) }\end{array}$} & \multicolumn{2}{|c|}{$\begin{array}{l}\text { Netzspinnen } \\
(\text { Sept. })^{\log }\end{array}$} \\
\hline & & $\mathrm{F}$ & $\mathrm{p}$ & $\mathrm{F}$ & $\mathrm{p}$ & $\mathrm{F}$ & $\mathrm{p}$ \\
\hline Modell & $7 ; 12$ & 4,83 & 0,0085 & 3,70 & $<0,05$ & 2,80 & $<0,10$ \\
\hline Ameise & $1 ; 12$ & 19,03 & 0,0009 & 1,06 & & 5,50 & 0,0370 \\
\hline Spinne & $1 ; 12$ & 0,003 & & 16,76 & 0,0015 & 0,08 & \\
\hline$A \times S$ & 1,12 & 3,87 & 0,0727 & 1,20 & & 0,11 & \\
\hline Block & $4 ; 12$ & 2,73 & 0,0792 & 1,72 & & 3,47 & $<0,05$ \\
\hline
\end{tabular}




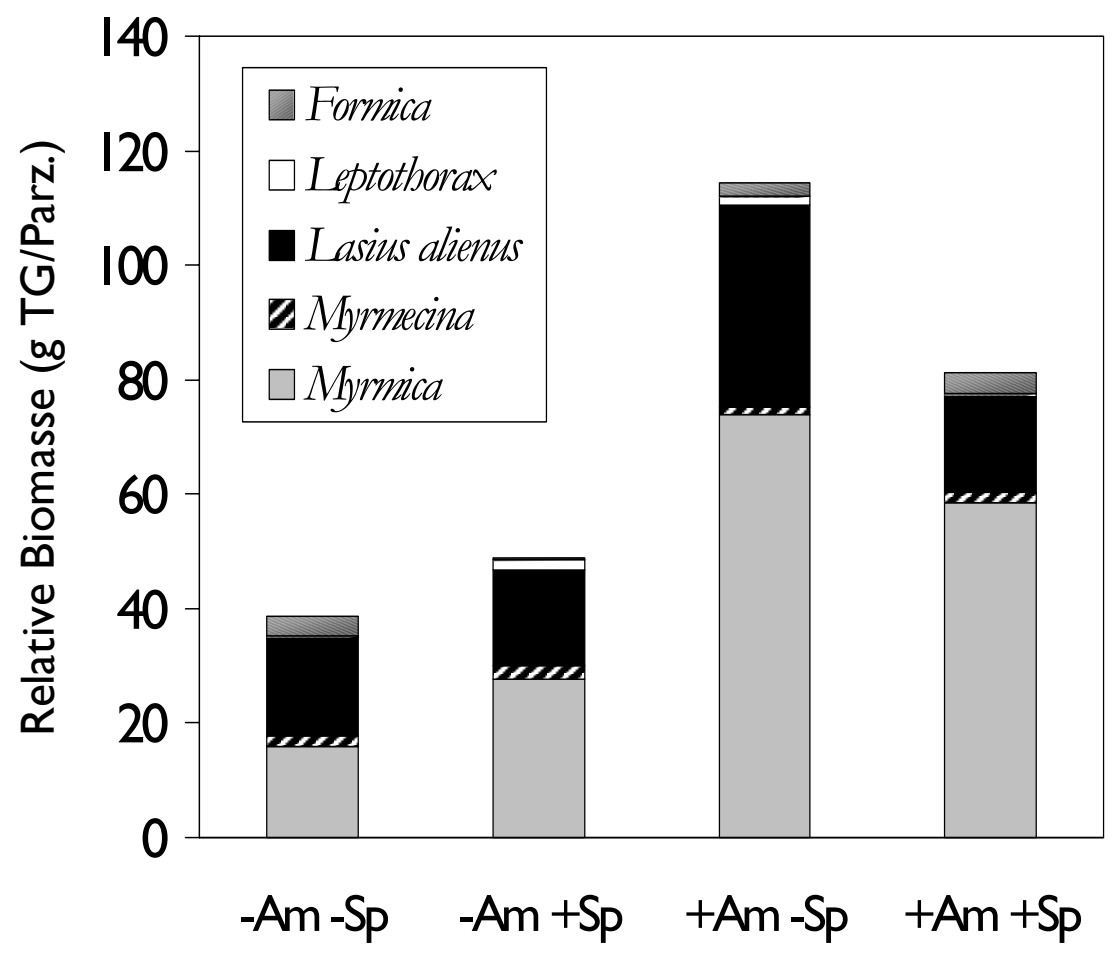

Abb. 45: Trockengewicht der epigäischen Ameisenarten Formica cunicularia, Leptothorax interruptus, Lasius alienus, Myrmecina graminicola und Myrmica sabuleti im Versuch mit experimentell veränderter Spinnen- bzw. Ameisendichte. Mittelwerte der Summen aller Beobachtungen und Proben aus jeder Parzelle. Am= Dichte von Ameisen reduqiert (-) bzw. erböbt (+); Sp= Dichte von Spinnen reduziert (-) bzw. erhöht (+).

\section{Spinnen}

Durch das Absammeln konnten vor allem die Dichte der vaganten Spinnen und auch ihre Biomassen signifikant reduziert werden (Abb. 46). Im Juni konnte die Biomasse der durch Saugfänge erfassten vaganten Spinnen in der entsprechenden Hälfte der Parzellen deutlich über das durchschnittliche Niveau der Umgebung hinaus erhöht werden; von August bis September reduzierte sich die Biomasse innerhalb der Parzellen wieder etwas, während sie außerhalb weiterhin anstieg (Abb. 46). Die Biomasse der vaganten Spinnen in den Bodenproben war durch die bessere Erfassung der Streuschicht höher als in den Saugfängen; die experimentelle Reduktion der Biomassen gelang für Vegetations- und Streuschicht zusammen nicht so deutlich. Etwa ein Fünftel der Biomasse aller vaganter Spinnen machte die Wolfsspinne Aulonia albimana aus. Ihre Biomasse konnte nur in der Variante mit gleichzeitig erhöhter Ameisendichte deutlich reduziert werden. 

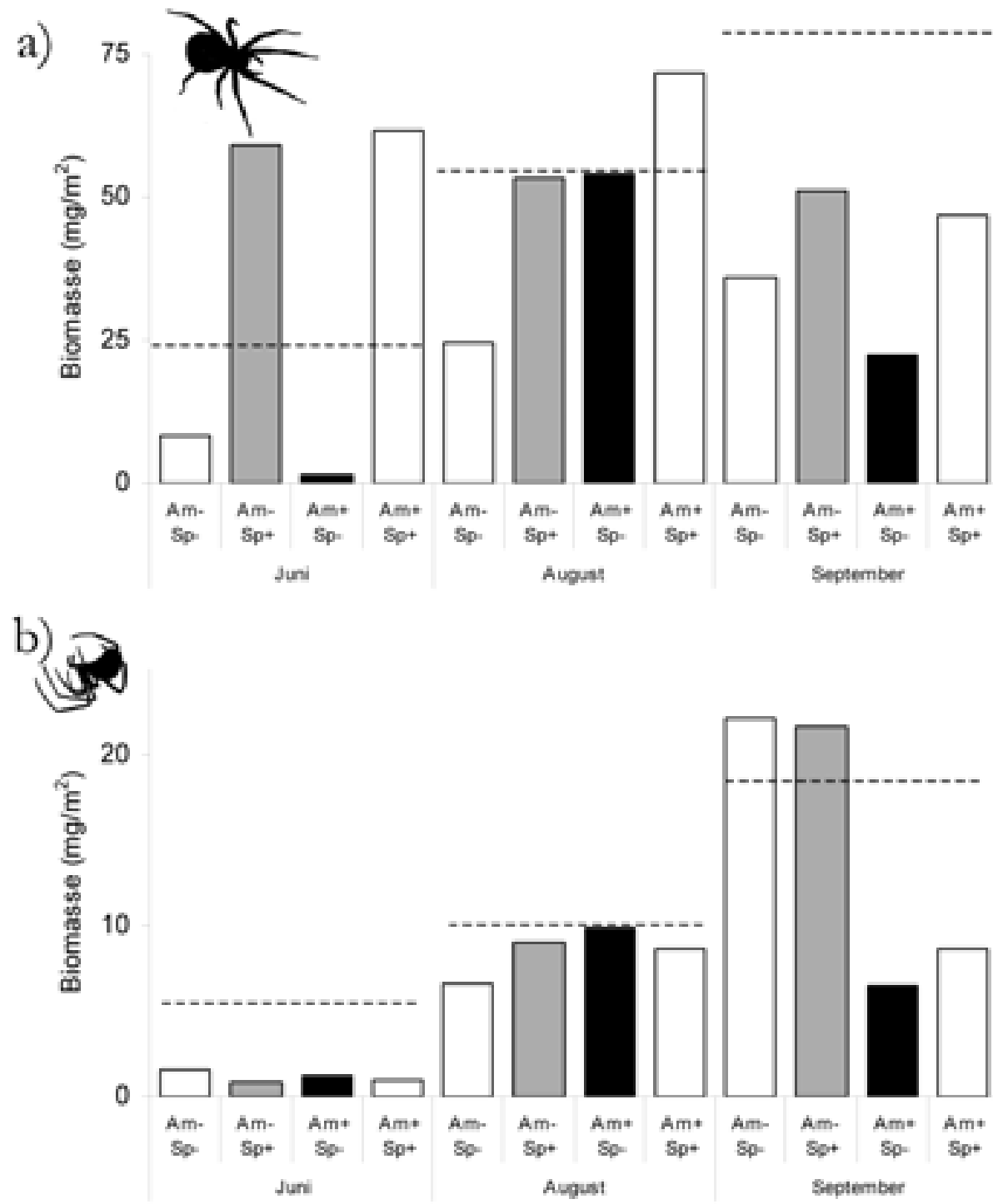

Abb. 46: Geometrische Mittelwerte der Biomassen der freijagenden (a) und netzbauenden (b) Spinnen der Vegetationsschicht im Versuch mit experimentell veränderter Spinnen- bzw. Ameisendichte aus den Saugfängen an drei Terminen im Sommer 2002. Am= Dichte von Ameisen reduziert (-) bzw. erhöbt (+); Sp= Dichte von Spinnen reduziert (-) bzw. erböht (+). ---: Spinnendichte außerbalb der Parzellen. Graphik verändert nach Sanders (2004).

Die Netzspinnen konnten in ihrer Dichte und Biomasse durch das Absammeln nicht signifikant beeinflusst werden. Die Individuenzahl der netzbauenden Spinnen nahm mit zunehmender Vegetationsdichte entlang des Hangs deutlich zu (vgl. Kap. 4). Die erhöhte Ameisenaktivität im Experiment hatte eine signifikante Re- 
duktion der Biomasse der Netzspinnen zur Folge, das Absammeln mit Reduktion der Biomasse der vaganten Spinnen führte zu einer signifikant höheren Individuendichte der juvenilen Spinnen aus den netzbauenden Familien in den Parzellen mit gleichzeitig reduzierter Zahl an Ameisennestern. In den nicht abgesammelten Parzellen mit reduzierter Ameisenaktivität hatten diese Spinnenarten die gleiche Biomasse bei deutlich niedrigerer Individuenzahl. Der negative Effekt der Ameisen auf die Netzspinnen wirkte sich auch auf die Zahl der Spinnennetze in den Parzellen aus: bei reduzierter Ameisendichte war die Zahl der Netze zum Beutefang in jeder Parzelle signifikant höher, in der Variante ohne gleichzeitiges Absammeln der Spinnen gab es etwa dreimal so viele Netze wie in den Varianten mit hoher Ameisenaktivität. Insbesondere die Baldachinnetzspinnen (Linyphiidae) reagierten mit geringeren Zahlen an Individuen und Netzen deutlich negativ auf höhere Ameisenbiomassen. Einige vagante Spinnen reagierten hingegen auf die Reduktion der Ameisendichte negativ. Springspinnen (Salticidae) und die Wolfsspinnengattungen Pardosa und Arctosa hatten in der Variante mit erhöhter Ameisenbiomasse ohne Absammeln der Spinnen das Maximum ihrer Dichte.

\section{Reaktion anderer Arthropodengruppen}

Deutliche Unterschiede in den Dichten von Isopoda, Diplopoda, Chilopoda, Collembola, Thysanoptera, Coleoptera, Diptera, Heteroptera, Auchenorrhyncha und Aphidoidea gab es meist nur zwischen den Blöcken. Alle Hemiptera und die Asseln hatten auf der Glatthaferwiese höhere Dichten als im Übergangsbereich zum Halbtrockenrasen. Die Lithobiidae hatten wie die vaganten Spinnen in der Mitte des Gradienten ihre höchsten Dichten.

Die unterschiedlichen Behandlungen der dominanten Prädatorengruppen hatten keine deutlichen Auswirkungen auf die übrige Fauna. Verschiedene Insektenlarven, hauptsächlich Zweiflügler-, Weichkäfer- und Schmetterlingslarven, hatten bei erhöhter Ameisenbiomasse geringere Dichten. Röhrenschildläuse (Ortheziidae), Fransenflügler (Thysanoptera) und Blattläuse (Aphidoidea) hatten an einzelnen Terminen signifikant höhere Dichten in den Parzellen mit höherer Ameisendichte. Springschwänze und Zikaden hatten bei reduzierter Spinnendichte höhere Dichten; dieser Effekt war aber nur für Collembola über $1 \mathrm{~mm}$ Körpergröße im Juni signifikant (Sanders 2004, Sanders \& Platner, unveröffentlicht).

Die Zikaden korrelierten mit der Netzspinnendichte und der Dichte von Lasius alienus an manchen Terminen positiv, mit der Dichte von Myrmica, Leptothorax und Formica eher negativ. Für die deutlichen Unterschiede in der Artengemeinschaft der Zikaden (Tab. 7 a, S. 48) ließ sich in einer multivariaten Ordination neben der Vegetationsdecke die Manipulation der Spinnendichte als strukturierender Faktor erkennen. Eine höhere Dichte epigäisch aktiver Ameisen mildert diesen Effekt vaganter Spinnen etwas ab. Mit höheren Spinnendichten waren die Schaumzikade Aphrophora alni und die Beilblattzikade Arboridia parvula assoziiert, mit geringeren die Erdzikade Aphrodes bicincta und die Spornzikaden Asiraca clavicornis, Stenocranus minutus und Hyledelphax elegantula (Rachor 2004). Wegen der stark 
geklumpten Verteilung der Zikaden ließen sich diese korrelativen Tendenzen allerdings nicht statistisch absichern.

\section{Geschlossene Parzellen}

In den nach dem Absaugen mit Gaze verschlossenen Parzellen konnten im Unterschied zu den offenen Parzellen mit regelmäßigem Absammeln schon nach vier Wochen keine signifikanten Unterschiede in der Spinnendichte mehr festgestellt werden (Sanders 2004). Die Dichte der Ameisen, Chrysomelidae, aller Spinnen und von Aulonia albimana einzeln war zwar tendenziell in den Parzellen mit anfangs erhöhter Spinnendichte höher, doch nur für die Blattläuse mit fünffach höherer Individuenzahl in den „Spinnenparzellen“ war dieser Unterschied signifikant. Zikaden und Schnurfüßer hatten tendenziell bei der anfangs vergröBerten Spinnenpopulation niedrigere Dichten. Die mittleren, maximalen und minimalen Temperaturen und die Luftfeuchtigkeit $5 \mathrm{~cm}$ über dem Boden zeigten ebenfalls keine Unterschiede zwischen den Parzellen mit und ohne Gazeabdeckung, allerdings deutliche zwischen den verschiedenen Hangabschnitten.

\section{Ameisenausschluss auf Bäumen und Sträuchern}

Der Leimring hat nach meinen Beobachtungen die Ameisen effektiv ausgeschlossen, vereinzelt auf solche Zweige gelangte Formica pratensis-Arbeiterinnen, die versuchten, wieder zurück zum Stamm zu gelangen, waren augenscheinlich nicht in der Lage, den Ring zu überwinden, so dass sie vermutlich von höher gelegenen Ästen auf die Äste mit dem Leimring gefallen waren. Zum Zeitpunkt der Probenahme war allerdings die Ameisenaktivität auf den Zweigen so gering, dass keine quantitativen Aussagen über sie gemacht werden können. Alle Bäume und Sträucher lagen im Aktivitätsgebiet einer Formica pratensis-Kolonie auf Fläche 1a (vgl. „Rasterarm“, Kap. 4) und wurden von Arbeiterinnen der Gattung Formica, von Lasius alienus und Myrmica sabuleti belaufen.

Fast alle untersuchten Tiergruppen hatten auf den Kiefernzweigen höhere Individuenzahlen als auf den Kirsch- oder Rosenzweigen (Abb. 47). Für die Spinnen, Blattläuse, Wanzen, Schmetterlingsraupen, Springschwänze und Milben war dieser Unterschied signifikant (Tab. 18).

Netzbauende Spinnen, Blattläuse und Blattflöhe, Käfer und Milben hatten auf den Ästen ohne Ameisenzugang höhere Dichten. Die Dichten der vaganten Spinnen, der Rindenläuse, Springschwänze, Schnecken, Rüssel- und Marienkäfer waren auf den Ästen mit Leimring niedriger (Abb. 47). Diese Unterschiede waren für Collembola und Psocoptera signifikant und traten bei den vaganten Araneida nur auf Kiefernzweigen auf (signifikante Interaktion). 

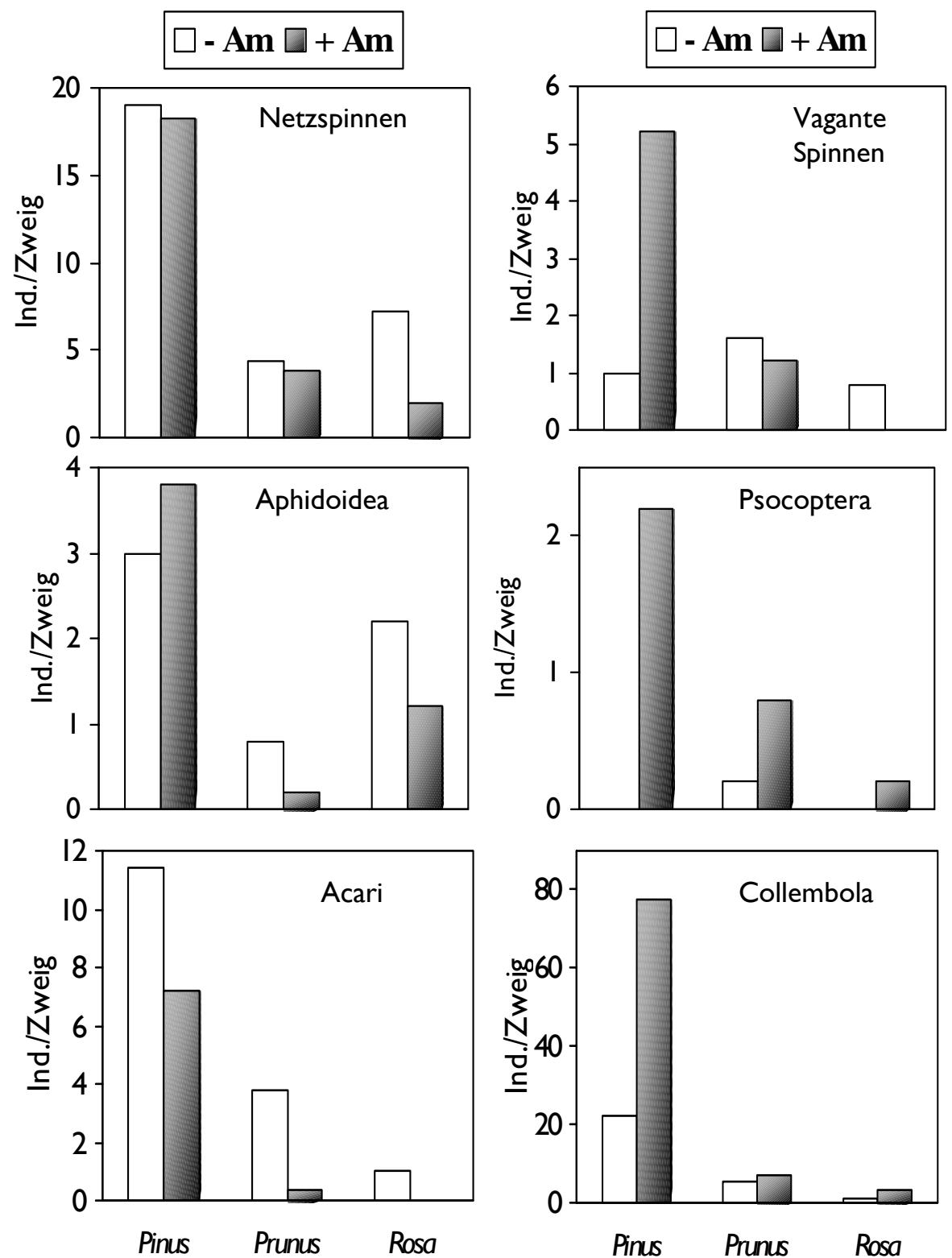

Gehölzart / Ameisenzugang

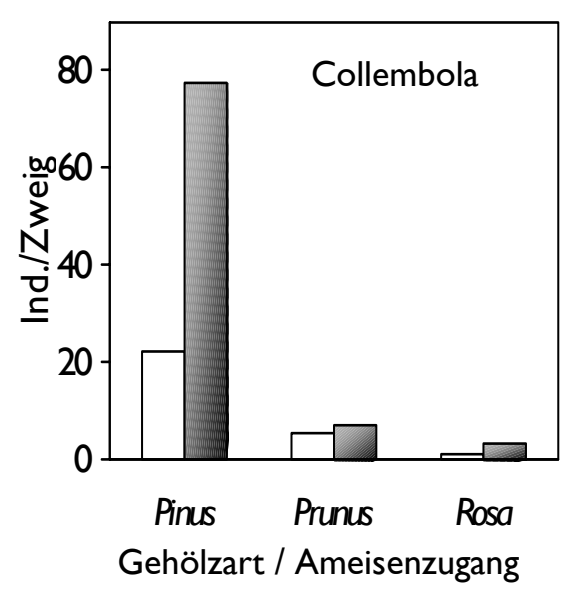

$A b b$. 47: Mittlere Individuenzablen der netzbauenden und freijagenden Spinnen, der Blatt- und Rindenläuse und der Mesofauna auf Zweigen mit experimentell verändertem Ameisenzugang von Kiefern (Pinus sylvestris), Süßkirschen (Prunus avium) und Hundsrosen (Rosa canina agg.) aus kombinierten Saug- und Klopffängen im August 2002. Am = Dichte von Ameisen reduziert (-) bəw. erböht (+). 
Tabelle 18: F-Werte (mit Freiheitsgraden [Effekt; Febler] als Index) und Wabrscheinlichkeiten für $\alpha$-Febler (p) der Effekte von experimentell verändertem Ameisenzugang und Gebölzart und deren Interaktionen aus zweifaktoriellen Varianzanalysen der Abundanzen von Spinnen, Pflanzenläusen und der Mesofauna auf Ästen gleicher Länge. $p<0,1$ : $p$-Werte fett gesetzt; log. Werte logtransformiert $\left(\log _{10} X+1\right)$.

\begin{tabular}{lcccccc} 
& \multicolumn{2}{c}{ Ameisen } & \multicolumn{2}{c}{ Gehölzart } & \multicolumn{2}{c}{$\mathrm{A} \times \mathrm{G}$} \\
& $\mathrm{F}_{1 ; 18}$ & $\mathrm{p}$ & $\mathrm{F}_{2 ; 18}$ & $\mathrm{p}$ & $\mathrm{F}_{2 ; 18}$ & $\mathrm{p}$ \\
\hline Netzspinnen & 0,99 & 0,3336 & 15,46 & $\mathbf{0 , 0 0 0 1}$ & 0,51 & 0,6098 \\
Vagante & 4,86 & $\mathbf{0 , 0 4 0 7}$ & 11,18 & $\mathbf{0 , 0 0 0 7}$ & 10,61 & $\mathbf{0 , 0 0 0 9}$ \\
Spinnen & & & & & & \\
Aphidoidea $^{\log }$ & 0,41 & 0,5289 & 5,15 & $\mathbf{0 , 0 1 7 0}$ & 0,00 & 0,9954 \\
Psocoptera $^{\log }$ & 4,68 & $\mathbf{0 , 0 4 0 7}$ & 1,31 & 0,2895 & 1,63 & 0,2162 \\
Acarina & 1,06 & 0,3129 & 3,79 & $\mathbf{0 , 0 3 7 2}$ & 0,12 & 0,8879 \\
Collembola & 3,82 & $\mathbf{0 , 0 6 2 3}$ & 9,05 & $\mathbf{0 , 0 0 1 2}$ & 3,12 & $\mathbf{0 , 0 6 2 3}$ \\
\hline
\end{tabular}

\subsection{Diskussion}

Die Ameisen hatten einen deutlichen negativen Einfluss auf die Netzspinnen und bei gleichzeitigem Absammeln der Spinnen auch auf die relativ sessile Wolfsspinne Aulonia albimana. Hingegen zeigten die Ameisen keine deutlichen Unterschiede in ihrer Dichte oder Aktivität zwischen den verschiedenen Behandlungen der Spinnendichte; nur die Arbeiterinnen der Arten Formica fusca und F. cunicularia waren in den Saugfängen aus Parzellen mit erhöhter Spinnendichte signifikant seltener vertreten als aus den Parzellen mit reduzierter Spinnendichte. Diese Arten sind sehr schnell und können sich sehr gut optisch orientieren, im Zusammentreffen mit anderen Arten verhalten sie sich auffallend rezessiv. Vermutlich bewirkt häufigeres Zusammentreffen mit größeren vaganten Spinnen auch häufigere Störung, so dass die Aufenthaltszeit der fouragierenden Arbeiterinnen und damit ihre Fangwahrscheinlichkeit in Parzellen mit hoher Spinnendichte entsprechend geringer ist.

In den Kronen von Douglasien, von denen Ameisen experimentell ausgeschlossen waren, wiesen Halaji et al. (1997) eine erhöhte Biomasse an Rindenläusen und einen Abundanz-Anstieg der vaganten Spinnen nach. Die Autoren vermuten, dass die verminderte Spinnendichte auf den Kontrollbäumen mit Ameisenzugang stärker durch direkte Interferenz-Konkurrenz als durch Prädation begründet ist. In diesem Versuch wurden gerade vagante Spinnen und Rindenläuse zusammen mit Springschwänzen häufiger auf den Zweigen gefunden, zu denen die Ameisen - und auch die Spinnen, Springschwänze und Rindenläuse selbst ungehinderten Zugang hatten. Von den vaganten Spinnen waren aber nur die Thomisidae auf den Kiefernzweigen deutlich häufiger; auf Kirsch- und Rosenzweigen hatten alle freijagenden Spinnen geringere Individuendichten. Von daher ist es wahrscheinlicher, dass der positive Effekt auf die Krabbenspinnen auf den 
Kiefernzweigen auf dem indirekten Einfluss der Ameisen beruht, als auf der direkten Wirkung des Leimrings, denn der gleiche Leimring war auch an den Kirschund Rosenzweigen angebracht. Bei Konkurrenz oder Intragildenprädation zwischen Netzspinnen und vaganten Jägern könnte der negative Effekt der Ameisen auf die Netzspinnen, der auch in der Streu- und Krautschicht nachgewiesen wurde, für einen positiven Effekt auf die Krabbenspinnen verantwortlich gewesen sein. Insbesondere Linyphiidae hatten bei Ameisenzugang niedrigere Dichten, Araneidae reagierten deutlich weniger; eine Dichtereduzierung von kleineren Krabbenspinnen oder auch Rindenläusen und Springschwänzen durch die Baldachinnetzspinnen ist nicht unwahrscheinlich. Die gleichsinnige Reaktion von Rindenläusen und vaganten Spinnen auf Ameisenausschluß in Nadelbäumen, die sowohl Halaji et al. (1997) als auch ich finden konnten, legt auch eine gewisse Ressourcenlimitierung der vaganten Spinnen auf Zweigen höherer Bäume nahe, die eine Vorbedingung für die Konkurrenz mit Netzspinnen durch Exploitation wäre. Diese Vermutung könnte durch Analyse der stabilen Isotope oder noch besser durch gezielte Experimente erhärtet werden.

Die Milben auf den Zweigen gehörten überwiegend prädatorischen Taxa an, so dass auch ein negativer Effekt der Ameisen auf die vermutlich leichter zu erbeutenden Milben für den positiven Effekt auf die Collembola und Psocoptera eine Rolle gespielt haben könnte.

Zwischen verschiedenen Ameisenarten erscheint Intragildenprädation eher unwahrscheinlich. Ameisen sind nicht nur für Spinnen sondern auch für unspezialisierte andere Ameisenarten keine leichte Beute - die hochgradig spezialisierten Amazonenameisen sind hier eine Ausnahme. Auch der in Ausschlussexperimenten nachgewiesene negative Effekt der dominanten Art Iridomyrmex sanguineus auf die diverse Ameisengemeinschaft in der australischen Savanne (Andersen \& Patel 1994) und der indirekte positive Effekt von Ameisen auf einen neotropischen Baum mit extrafloralen Nektarien (de la Fuente \& Marquis 1999) beruhen vermutlich eher auf permanenter Störung der subdominanten Ameisenarten, bzw. der Phytophagen, als auf Fraßdruck. Der Einfluß der ebenfalls dominanten Art Iridomyrmex purpurens ist beispielsweise auch nur auf ökologisch ähnliche Arten der gleichen Gattung beschränkt (Gibb \& Hochuli 2004). 


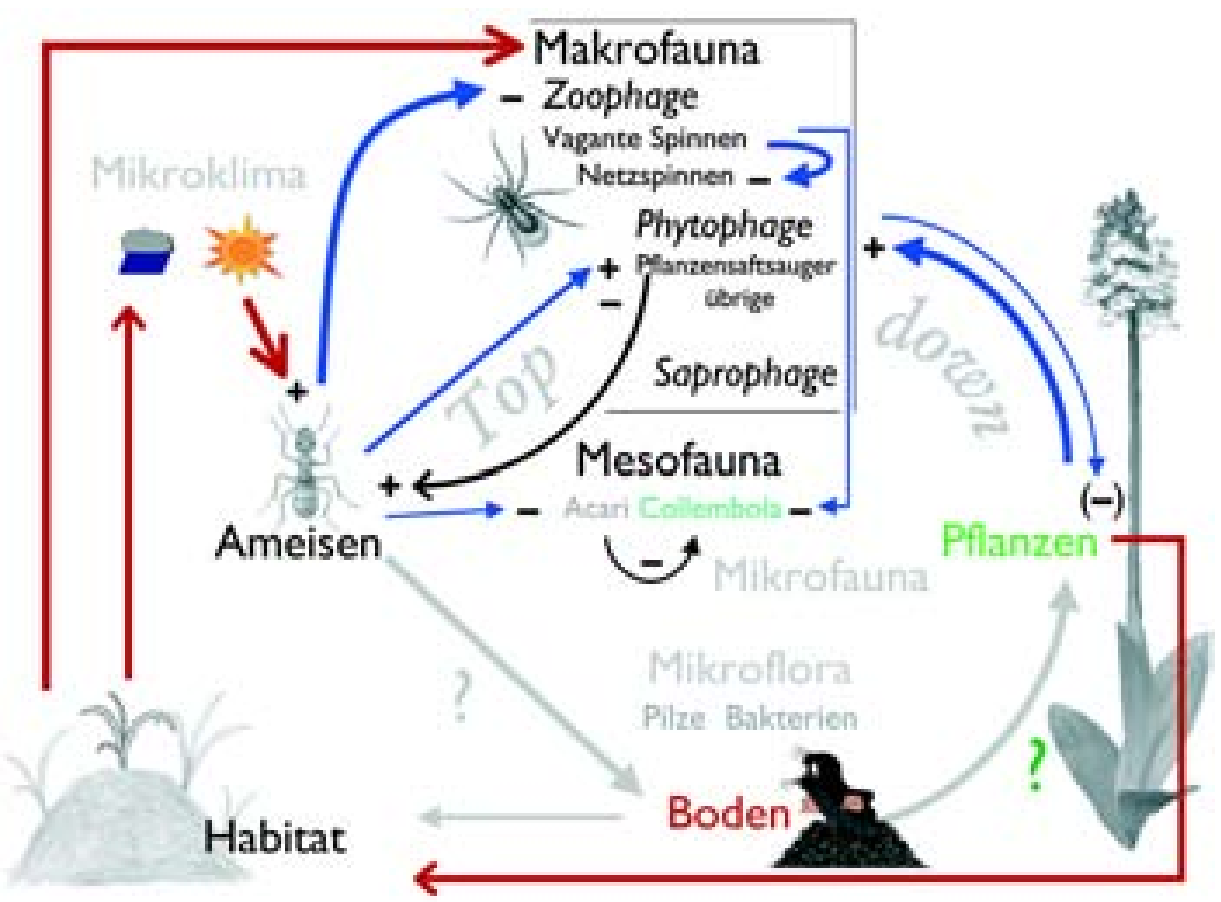

Abb. 48: Oberirdische Wechselwirkungen zwischen Ameisen, Pflanzen, Makro- und Mesofauna, wie sie im Labor- und Freilandversuch nachgewiesen wurden. 


\section{Themenbereich III. \\ Beeinflussung des Bodens \\ und Wirkungen im Ökosystem}

\section{Biotische und abiotische Bodenveränderungen und deren bottom-up Wirkung auf das Wachstum verschiedener Pflanzen}

\subsection{Einleitung}

Ameisen können die Ressourcenverfügbarkeit für andere Organismen durch direkte und indirekte Veränderungen der physischen, chemischen und biologischen Umwelt im Boden wirksam modulieren. Die vorwiegend unterirdisch lebende, hügelbauende gelbe Wiesenameise Lasius flavus hat in vielen offenen Lebensräumen durch ihre großen Biomassen (bis zu $150 \mathrm{~kg}$ Lebendgewicht/ha) und intensive Grabtätigkeiten (jährlich bis zu mehreren Tonnen Boden pro Hektar) einen starken Einfluss auf die Bodenbedingungen und auch auf Bodentiere und mikroorganismen (Waloff \& Blackith 1962, Nielsen et al. 1976, Dlussky 1981, Seifert 1996). Auf älteren Brachflächen kann die gesamte obere Bodenschicht durch Ameisen umgewandelt worden sein (Woodell \& King 1991). Der Boden aus Nestern von Lasius flavus weist im Vergleich zum umgebenden Boden deutliche Unterschiede in Wasserhaushalt, Bodenchemie und der mikrobiellen Gemeinschaft auf (Pętal 1978, Beattie 1989, Lobry de Bruyn \& Conacher 1994, Dean et al. 1997, Dauber \& Wolters 2000).

Diese wichtige Funktion der Ameisen hat verglichen mit Regenwürmern oder Termiten bisher relativ wenig Beachtung in der ökologischen Forschung gefunden, insbesondere die Einflüsse auf die Bodenfauna wurden erst in letzter Zeit und 
detaillierter nur bei Ernte- und Waldameisen untersucht (Wagner et al. 1997, Laakso \& Setälä 1998). Blomqvist et al. (2000) konnten in Kolonien von Lasius flavus einen deutlich niedrigeren Anteil an pflanzenparasitischen Nematoden als im umgebenden Boden einer leicht sauren, nährstoffarmen, feucht-sandigen Wiese beobachten.

Diese teilweise drastischen Änderungen in der Bodenchemie sollten auch die Ressourcen-Verfügbarkeit für Pflanzen ändern und damit direkten Einfluss auf die Wuchsleistungen verschiedener Pflanzen haben. Dieser bottom-up-Einfluss der Ameisen wurde im Labor mit einem Mikrokosmosexperiment genauer analysiert.

\subsection{Methodik}

\section{Freilanduntersuchungen}

Im Werratal bei Witzenhausen-Freudenthal wurden auf drei benachbarten, brachliegenden Glatthaferwiesen auf Muschelkalk an fünf Terminen in wöchentlichem Abstand im Sommer 1999 insgesamt 30 Hügelnester von Lasius flavus und der umgebende Wiesenboden in je $1 \mathrm{~m}$ Abstand in einem balancierten Blockdesign beprobt.

Wiese 1, Wiese 2 und Wiese 3 wurden vorher in jeweils zwei Teilflächen unterteilt:

- Wiese 1 in das Glatthaferwiesenstück direkt vorm Feldahorn-Hain (vgl. Kap. 4) und das etwas oberhalb davon liegende Stück, das langsam in den Halbtrockenrasen übergeht, auf dem drei Jahre später auch der Prädatoren-Ausschluss-Versuch durchgeführt wurde (Kap. 7);

- Wiese 2 in Block 5 des Klimamanipulationsversuchs (Kap. 5), auf dem noch genügend unmanipulierte Nesthügel vergleichbarer Größe waren, und einen südlich daran angrenzenden Teil der benachbarten Streuobstwiese (vgl. Abb. 2);

- Wiese 3 in die westliche und östliche Hälfte, die durch einige alte Süßkirschen und ein kleineres Schlehengebüsch getrennt sind.

An jedem Termin wurden auf jeder dieser sechs Teilflächen jeweils ein anderer zufällig ausgewählter Nesthügel und eine Kontrollfläche beprobt. Dazu wurden mit einem $10 \mathrm{~cm} \varnothing$-Stechzylinder Bodenproben (0-20 cm Tiefe) entnommen, vor Ort gesiebt $(4 \mathrm{~mm})$ und durchmischt. Im Labor wurden die Wassergehalte gravimetrisch (Trocknung bei $105^{\circ} \mathrm{C}, 24 \mathrm{~h}$ ), Kohlenstoff- und Stickstoffgehalte gaschromatographisch, Ammonium- und Nitratgehalte durch KjeldahlDestillation, Phosphatgehalte photometrisch und die $\mathrm{pH}$-Werte potentiometrisch bestimmt. Die Basalatmung und die mikrobielle Biomasse wurden durch SubstratInduzierte Respiration (vgl. Kap. 3.3.) gemessen. Die Nematoden wurden mit einer modifizierten Baermann-Nassextraktion (Alphei 1995) ausgetrieben.

Mit einer Varianzanalyse wurde der Einfluss der unabhängigen Faktoren „Ameisennest“ und „Termin“ statistisch überprüft. Da man bei einem Meter Ab- 
stand schon eher von zwei unabhängigen Teilproben ausgehen kann (vgl. Kap. 5) und die Verbundenheit erst auf einem Skalenniveau höher wieder stärker wird (vgl. Flächen- bzw. Blockeffekte in Kap. 4, 5 und 7), habe ich die durch unterschiedliche Umgebung hervorgerufene Varianz durch einen Block- und einen darin geschachtelten Sub-Block-Effekt berücksichtigt.

\section{Laborversuch}

Drei Pflanzenarten aus verschiedenen funktionellen Gruppen (Gras, Kraut, Leguminose) wurden in einer Klimakammer einzeln und in Konkurrenz miteinander in Baumaterial aus Lasius flavus - Nestern und im Vergleich in Kontrollboden aus dem Untersuchungsgebiet angezogen (Abb. 49). Der entnommene Boden wurde nach einer Austreibung der Tiere durch Hitzeextraktion gesiebt (4 mm), gemischt und anschließend in je 48 Labor-Mikrokosmen gefüllt. In je 6 dieser Mikrokosmen wurden Weißklee (Trifolium repens), Glatthafer (Arrhenatherum elatius) und WiesenLabkraut (Galium mollugo) einzeln und in faktorieller Kombination gepflanzt.

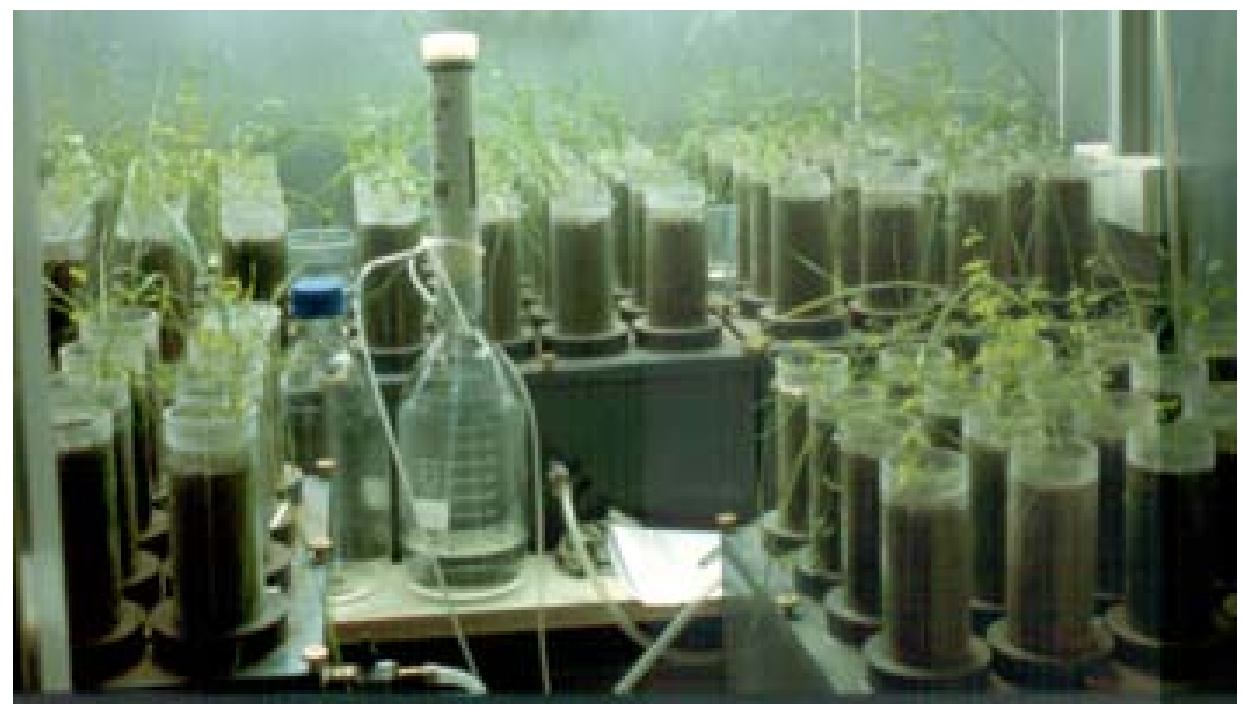

Abb. 49: Mikerokosmen mit Weißklee, Glatthafer und Wiesenlabkeraut einzeln und in Konkurrenz in Boden aus Nestern von Lasius flavus und von der umgebenden Wiese.

Die ober- und unterirdischen Biomassen der einzelnen Pflanzen wurden nach Waschen der Wurzeln und Trocknung $\left(24 \mathrm{~h}, 105^{\circ} \mathrm{C}\right)$ gewogen. Die Kohlenstoffund Stickstoffgehalte des Bodens, der einzelnen Pflanzenteile und des Eluats wurden gaschromatographisch, Ammonium- und Nitratgehalte durch KJELDAHLDestillation, Phosphatgehalte photometrisch und die $\mathrm{pH}$-Werte potentiometrisch gemessen.

Der Einfluss der unabhängigen Faktoren „Ameisennest“, „Gras“, „Kraut“ und „Leguminose“ wurde mit einer Varianzanalyse statistisch überprüft. 


\subsection{Ergebnisse}

\section{Bodenveränderungen}

Nach zweiwöchiger Trockenheit zu Beginn der Messperiode hatte der Boden der Nesthügel einen deutlich geringeren Wassergehalt als der umgebende Boden. Nach kräftigen Niederschlägen am 5. August stiegen beide Werte an; nach einer weiteren Woche mit z.T. weiteren kräftigen Regengüssen wurde der Boden aus den Nestern sogar deutlich feuchter als der Kontrollboden (Abb. 50). Der Effekt der Ameisen auf den Wassergehalt des Bodens war folglich stark terminabhängig (signifikante Interaktion der Faktoren) und führte insgesamt zu stärkeren Schwankungen mit größeren Extremen.

Die $\mathrm{pH}$-Werte der drei Wiesen unterschieden sich signifikant, die Ameisen hatten aber offensichtlich keinen Einfluss darauf. Auch die Kohlenstoff- und Stickstoffgehalte unterschieden sich signifikant zwischen den drei Wiesen, unterlagen aber auch einem deutlichen Einfluss der Ameisennester: in den Nestern waren die Stickstoffgehalte signifikant höher und die Kohlenstoffgehalte signifikant niedriger als im umgebenden Boden. Die Kohlenstoffgehalte stiegen sowohl im Nestals auch im Kontrollboden nach den Niederschlägen deutlich an. Entsprechend haben die Faktoren „Ameisennest“, „Termin“ und „Block“ auch einen signifikanten Einfluss auf das C/N-Verhältnis, das in den Nestern deutlich niedriger war.

Die Gehalte an löslichen Phosphat-, Nitrat- und Ammoniumionen waren im Boden der Nesthügel gegenüber dem umgebenden Boden signifikant sehr stark erhöht (Abb. 50).

Auch in den verschiedenen Mikroklimavarianten des Zusatzversuches zur Temperaturmessung (vgl. S. 59) waren zwei Wochen nach den letzten Niederschlägen die Nesthügel signifikant trockener als der umgebende Wiesenboden, allerdings nur in den oberen Zentimetern (Tab. 19 und 20). Die Basalatmung war entsprechend auch nur im oberen Horizont des Wiesenbodens höher als im Nesthügel. Die mikrobielle Biomasse war insgesamt auch in den Nestern mit verändertem Mikroklima signifikant erhöht; dieser Effekt beruhte aber nur auf dem erheblichen Unterschied in 12 bis $15 \mathrm{~cm}$ Tiefe (hochsignifikante Interaktion Horizont x Nest, Tab. 20). Zusätzliche Beregnung hat zwar den Wassergehalt in der Sonne kurzzeitig (vgl. Abb. 26), im Schatten auch länger erhöht; auf die Basalatmung hatte aber v.a. die Austrocknung eine negative Wirkung. Die mikrobielle Biomasse wurde durch Beschattung insbesondere im oberflächlichen Ameisennestboden erhöht.

Die Ergosterolgehalte als Maß der pilzlichen Biomasse waren in beiden Bodenhorizonten in den Ameisennestern deutlich höher als im umgebenden Wiesenboden (Tab. 19 und 20). Zusätzliche Beregnung hat das Ergosterol im Boden signifikant verringert. Der hohe Ergosterolgehalt und die niedrige spezifische Respiration im oberflächlichen Boden vom Ameisennest weist trotz stärkerer zeitlicher Schwankungen (Abb. 51) auf insgesamt bessere Bedingungen für die Mikroflora hin als im Wiesenboden. 

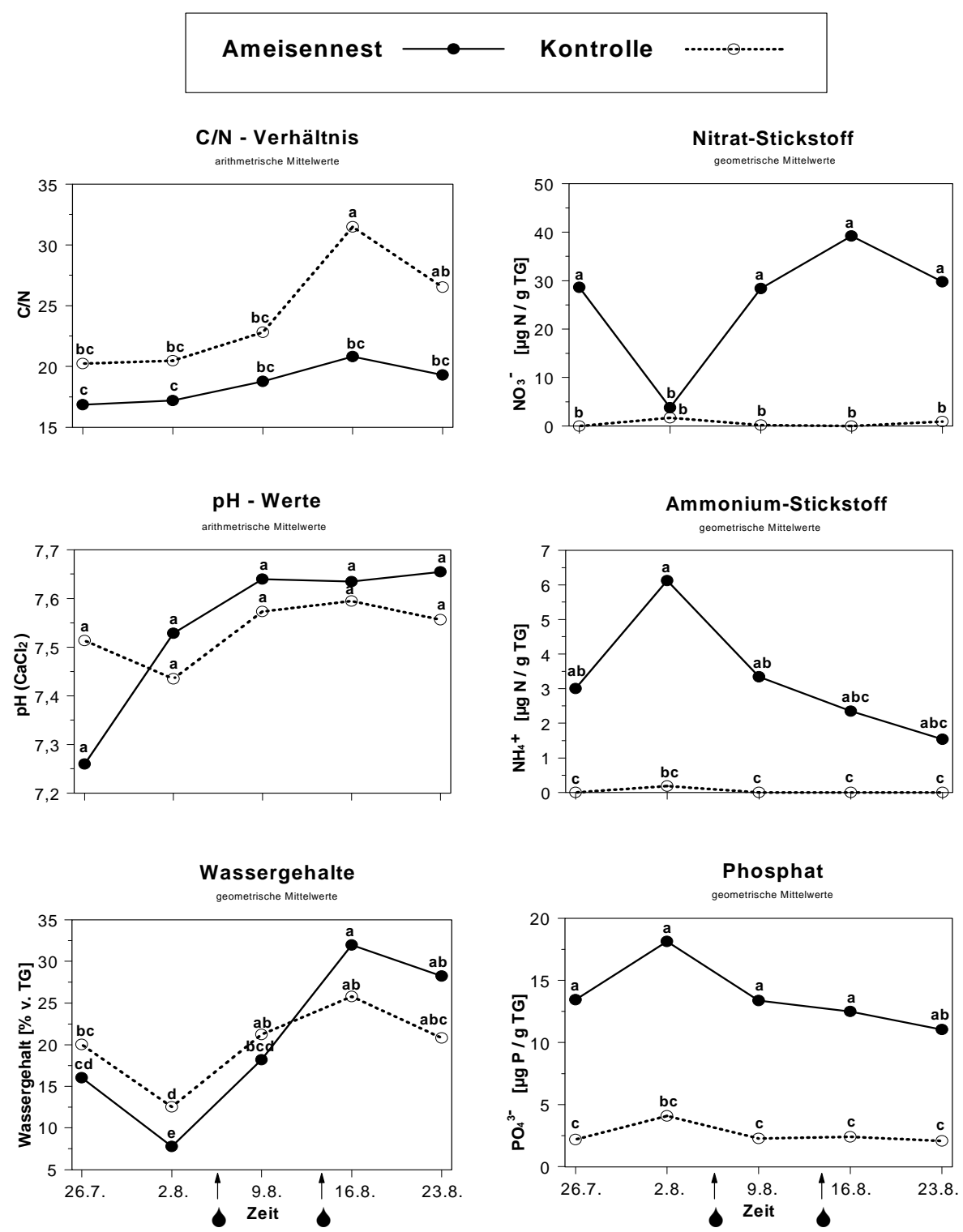

Abb. 50: Mittlere C/N-Verbältnisse, Wasser-, Protonen- und Nährstoffgehalte der Bodenproben aus den Nestern von Lasius flavus (•) und dem umgebenden Wiesenboden (O) an den fünf Messterminen.

$\boldsymbol{\vartheta}=$ Niederschläge über $25 \mathrm{~mm}$. Unterschiedliche Buchstaben zeigen signifikant unterschiedliche Mittelwerte an (Tukey-Test). 
Auswirkungen auf andere Bodenbewohner

Die Basalatmung und die mikrobielle Biomasse waren im Ameisennest-Boden signifikant über den gesamten Untersuchungszeitraum stark erhöht; sie zeigten dabei aber auch entsprechend den Schwankungen des Wassergehalts eine deutliche zeitliche Dynamik. Das Verhältnis der mikrobiellen Biomasse zum Gesamtkohlenstoff (Cmic/Corg-Verhältnis) im Boden aus den Ameisennestern war im Mittel mit 0,0138 höchst-signifikant größer als im Kontrollboden mit 0,0058; die spezifische Respiration war besonders zu Beginn im Nestboden deutlich niedriger, näherte sich aber durch die deutlich erhöhte Basalatmung nach den Niederschlägen derjenigen vom Wiesenboden an (Abb. 51). Die Atmungskurven der Bodenproben aus den einzelnen Nestern zeigten nach Glucose-Zugabe grundlegende Unterschiede. Neben einfach erhöhten Kurven ohne erkennbare Wachstumsphase traten sowohl eingipfelige als auch zweigipfelige Wachstumskurven auf. Trotz deutlicher Fluktuation war die Dichte der Fadenwürmer (Nematoda) in den Ameisennestern im Mittel über alle Termine signifikant höher (signifikanter Ameisenund Termineffekt).

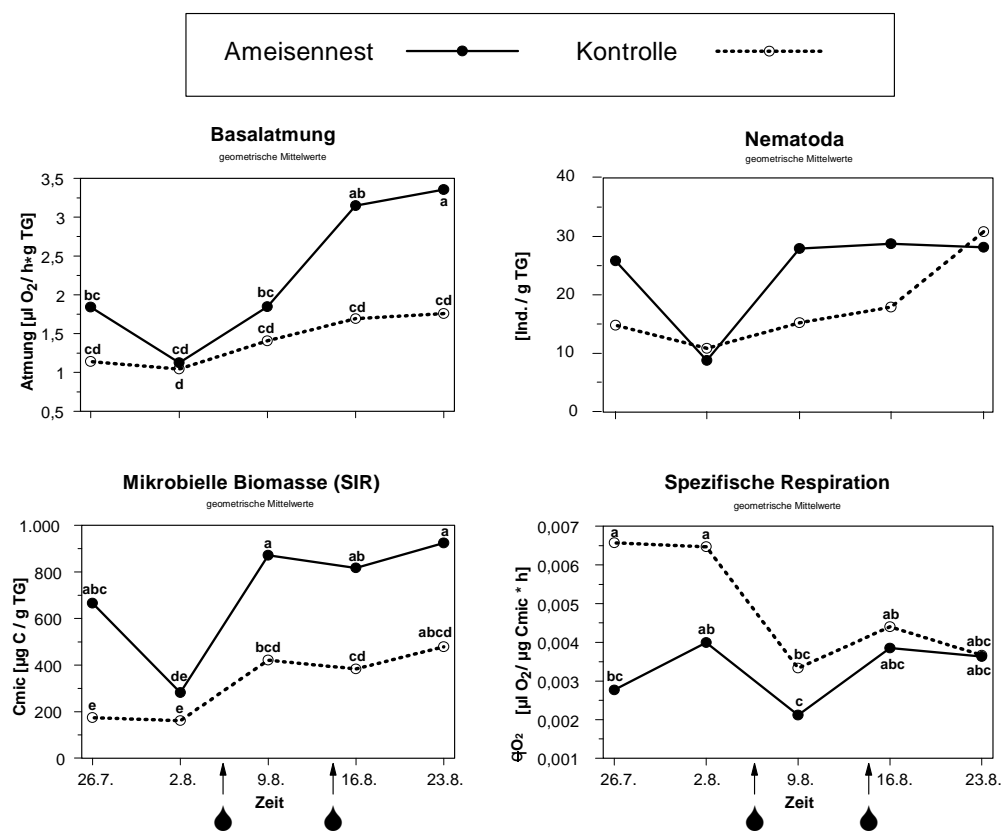

Abb. 51: Mittlere Basalatmung, mikrobielle Biomasse, sperifische Respiration und Nematodendichte der Bodenproben aus den Nestern von Lasius flavus (•) und dem umgebenden Wiesenboden (O) an den fünf Messterminen. = Niederschläge über $25 \mathrm{~mm}$. Unterschiedliche Buchstaben zeigen signifikant unterschiedliche Mittelwerte an (Tukey-Test). 
Tabelle 19: Wassergehalte, Basalrespiration, mikrobielle Biomasse und Ergosterolgehalte von Bodenproben aus Wiesenboden und Nestern von Lasius flavus vom 4.9.2002, jeweils aus 0 bis 3 $\mathrm{cm}$ und 12 bis $15 \mathrm{~cm}$ Tiefe. Mittelwerte $\pm 1 S E$ über sechs verschiedene Mikeroklimavarianten und zwei Blöcke (vgl. Kap. 5 \& Abb. 26); Interaktionen vgl. Tab. 20.

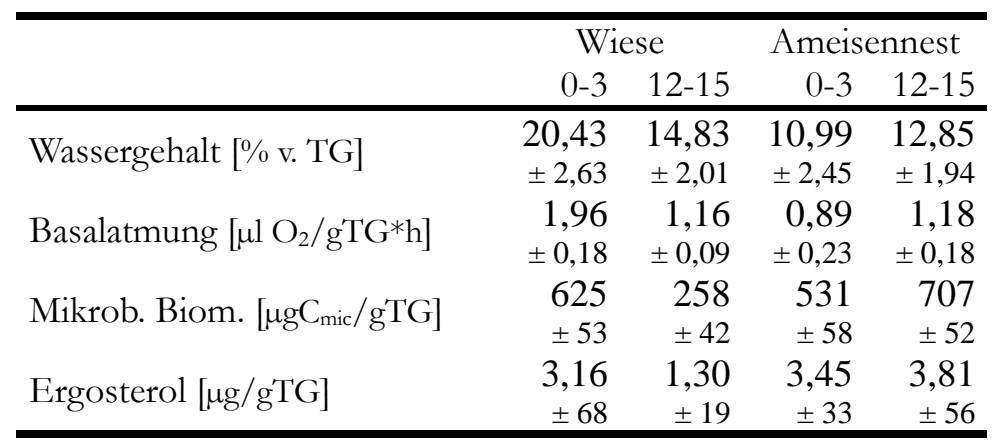

Tabelle 20: F-Werte und Wabrscheinlichkeiten für $\alpha$-Fehler (p) der Ameisennest-, Mikroklima-, Block- und Horizonteffekte und deren Interaktionen aus dreifaktoriellen Varianzanalysen für Messwiederholungen der Wassergehalte, Basalatmungen, mikrobiellen Biomassen (Cmic) und Ergosterolgehalte ein Vierteljahr nach Beginn der Mikroklimamanipulation. FG: Freiheitsgrade (Effekt; Fehler); Horizonteffekte: F-Werte des multivariaten Pillai trace Tests; $p<0,05:$ F-Werte fett

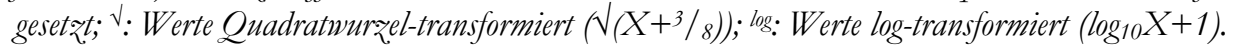

\begin{tabular}{|c|c|c|c|c|c|c|c|c|c|}
\hline & \multirow[b]{2}{*}{$\mathrm{FG}$} & \multicolumn{2}{|c|}{ Wassergeh. $^{\vee}$} & \multicolumn{2}{|c|}{ Basalresp. $\log$} & \multicolumn{2}{|c|}{$\mathrm{Cmic}^{\sqrt{ }}$} & \multicolumn{2}{|c|}{ Ergosterollog } \\
\hline & & $\bar{F}$ & $p$ & $\bar{F}$ & $p$ & $\mathrm{~F}$ & $p$ & $\mathrm{~F}_{\mathrm{FG}}$ & $\bar{p}$ \\
\hline Regen & $2 ; 11$ & 8,49 & 0,0059 & 34,66 & $<, 0001$ & 0,07 & 0,9369 & 4,33 & 0,0410 \\
\hline Sonne & $1 ; 11$ & 34,24 & 0,0001 & 34,37 & 0,0001 & 3,86 & 0,0752 & 3,26 & 0,0986 \\
\hline Nest & $1 ; 11$ & 14,96 & 0,0026 & 60,42 & $<, 0001$ & 9,13 & 0,0116 & 39,07 & $<, 0001$ \\
\hline $\mathrm{R} \times \mathrm{S}$ & $2 ; 11$ & 2,88 & 0,0988 & 1,90 & 0,1957 & 0,52 & 0,6061 & 3,35 & 0,0729 \\
\hline $\mathrm{R} \times \mathrm{N}$ & $2 ; 11$ & 0,49 & 0,6228 & 16,62 & 0,0005 & 0,46 & 0,6435 & 0,30 & 0,7483 \\
\hline $\mathrm{S} \times \mathrm{N}$ & $1 ; 11$ & 1,18 & 0,3007 & 19,38 & 0,0011 & 0,09 & 0,7653 & 0,20 & 0,6599 \\
\hline $\mathrm{R} \times \mathrm{S} \times \mathrm{N}$ & $2 ; 11$ & 0,76 & 0,4920 & 2,96 & 0,0933 & 3,00 & 0,0911 & 0,46 & 0,6413 \\
\hline Block & $1 ; 11$ & 4,87 & 0,0495 & 0,18 & 0,6799 & 0,29 & 0,6033 & 13,96 & 0,0033 \\
\hline Horizont & $1 ; 11$ & 1,12 & 0,3122 & 0,01 & 0,9436 & 15,87 & 0,0021 & 40,61 & $<, 0001$ \\
\hline $\mathrm{H} \times \mathrm{R}$ & $2 ; 11$ & 2,01 & 0,1805 & 9,04 & 0,0048 & 0,13 & 0,8803 & 1,23 & 0,3286 \\
\hline $\mathrm{H} \times \mathrm{S}$ & $1 ; 11$ & 1,17 & 0,3017 & 10,11 & 0,0088 & 4,62 & 0,0547 & 0,45 & 0,5161 \\
\hline $\mathrm{H} \times \mathrm{N}$ & $1 ; 11$ & 20,78 & 0,0008 & 113,78 & $<, 0001$ & 91,64 & $<, 0001$ & 51,25 & $<, 0001$ \\
\hline $\mathrm{H} \times \mathrm{R} \times \mathrm{S}$ & $2 ; 11$ & 0,46 & 0,6420 & 2,58 & 0,1203 & 0,35 & 0,7097 & 4,29 & 0,0419 \\
\hline $\mathrm{H} \times \mathrm{R} \times \mathrm{N}$ & $2 ; 11$ & 1,12 & 0,3609 & 0,14 & 0,8668 & 2,32 & 0,1445 & 2,49 & 0,1283 \\
\hline $\mathrm{H} \times \mathrm{S} \times \mathrm{N}$ & $1 ; 11$ & 3,43 & 0,0912 & 0,02 & 0,8881 & 9,79 & 0,0096 & 1,91 & 0,1944 \\
\hline $\mathrm{H} \times \mathrm{R} \times \mathrm{S} \times \mathrm{N}$ & $2 ; 11$ & 1,31 & 0,3094 & 4,77 & 0,0322 & 0,27 & 0,7716 & 1,53 & 0,2587 \\
\hline $\mathrm{H} \times \mathrm{Bl}$ & $1 ; 11$ & 5,65 & 0,0367 & 0,86 & 0,3731 & 3,44 & 0,0907 & 1,63 & 0,2282 \\
\hline
\end{tabular}



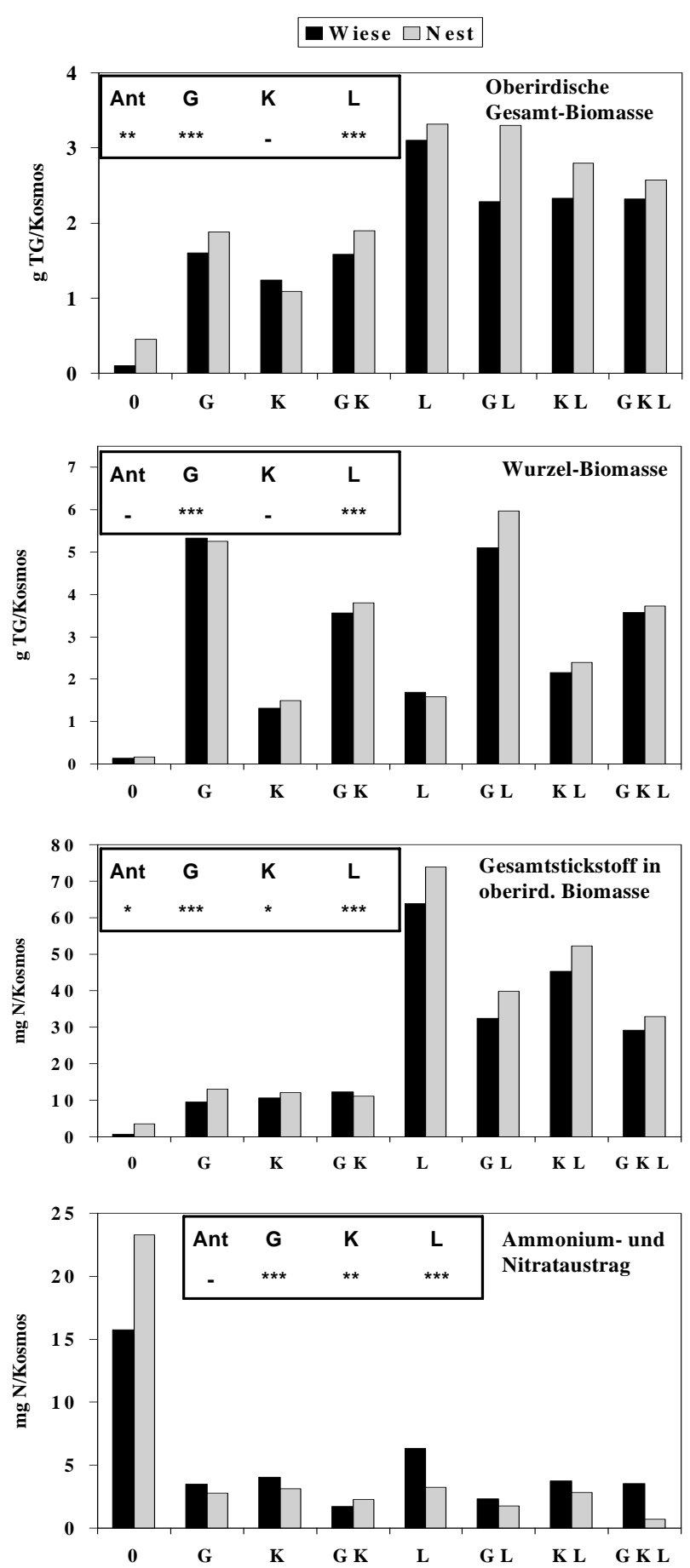

Die jeweiligen Pflanzenarten hatten unterschiedliche Effekte auf die untersuchten Parameter; steigende Pflanzendiversität führte dabei durchschnittlich zu höheren Gesamt-Wuchsleistungen. In den Varianten mit Boden aus Ameisennestern war die oberirdische Phytobiomasse signifikant im Vergleich zu denen mit umgebenden Wiesenboden erhöht (Abb. 52).

Zwischen den Bepflanzungsvarianten und der Herkunft des Bodens zeigten sich deutliche Interaktionen, besonders in den Stickstoffbilanzen der einzelnen Systeme. In den Varianten mit Kleebepflanzung wurde eine größere Stickstoffmenge in der oberirdischen Biomasse angelegt, was bei Boden aus Ameisennestern noch verstärkt wurde.

$A b b$. 52: Pflanzliche oberund unterirdische Gesamtbiomassen, Gesamt-Stickstoffmengen in den oberirdischen Pflanzenteilen und im Eluat $\left(\mathrm{NH}_{4}{ }^{+}, \mathrm{NO}_{3}{ }^{-}\right)$; jenveils mit Signifikanzniveaus für die getesteten Faktoren (ANOVA: *: $\quad p<0,05$; **: $p<0,01$; ***: $p<0,001$; 6 Parallelen). 0: Kontrolle, G: Gras, K: Kraut, L: Leguminose, Ant: Ameisennest. 
Der Stickstoffaustrag wurde durch alle drei Pflanzenarten signifikant reduziert; aus den Mikrokosmen mit Ameisennestboden wurde ohne Bepflanzung mehr, mit Bepflanzung jedoch weniger Stickstoff ausgewaschen als aus denen mit Vergleichsboden (Abb. 52).

Die Nitratgehalte des Bodens waren in den Mikrokosmen mit Ameisennestboden nach dem Experiment signifikant niedriger, die Phosphatgehalte blieben trotz signifikant stärkerer Auswaschung höher als im Wiesenboden. Der Gesamt-Stickstoffgehalt des Bodens erhöhte sich im Ameisennestboden während des Experiments, im Wiesenboden hingegen sank er.

\subsection{Diskussion}

Die Ameisen riefen sowohl klare abiotische als auch deutliche biotische Bodenveränderungen hervor. Die Erde aus den Bauten hatte signifikant höhere Gehalte an verfügbarem Stickstoff und Phosphor; der Gesamt-Stickstoffgehalt war signifikant erhöht, während der Gesamt-Kohlenstoffgehalt niedriger war als im Vergleichsboden. Dean et al. (1997) fanden im Boden aus Nesthügeln von Lasius flavus- Kolonien hingegen niedrigere Gesamt-Stickstoffgehalte als im Vergleichsboden. Die auch nach restloser Entkalkung noch deutlich niedrigeren Kohlenstoffgehalte deuten auf eine erhöhte C-Mineralisationsrate hin, wie sie Dauber und Wolters (2000) für Lasius flavus Nester dokumentierten. Der Anstieg der Kohlenstoffgehalte nach den Niederschlägen könnte durch verstärktes Pflanzenwurzelwachstum (vgl. Platner et al. 1999) hervorgerufen worden sein.

Die chemischen und mikrobiellen Analysen belegen für den Boden aus den Ameisennestern eine deutlich bessere Ressourcenverfügbarkeit und -nutzung durch Mikroorganismen; die Atmungskurven geben einen deutlichen Hinweis auf Unterschiede in der Diversität der mikrobiellen Gemeinschaft sowohl innerhalb der Nester als auch zwischen ihnen.

Ameisennester sind generell wichtige Mikrohabitate für die Mesofauna. Viele Milben scheinen eher allgemein, Springschwänze eher partiell günstigere Bedingungen als im umgebenden Boden vorzufinden (Kap. 9; Tittel, Platner \& Migge, unveröffentlicht). Wagner et al. (1997) fanden in nordamerikanischen Nestern von Pogonomyrmex barbatus 5-fach erhöhte Protozoen- und 30-fach erhöhte MesofaunaDichten, Laakso \& Setälä stellten in finnischen Nestern von Formica aquilonia 7fach höhere Regenwurm-Biomassen (1997) und eine deutlich veränderte Zusammensetzung des gesamten Boden-Nahrungsnetzes (1998) fest, jeweils im Vergleich zum umgebenden Boden. Auch Nematoden sind in den Nesthügeln, zumindest im Wald und auf trockenen Brachen, häufiger - die funktionellen Gruppen scheinen dabei deutlich unterschiedlich zu reagieren: Bakterienfresser scheinen sehr positiv, Pilzfresser und Pflanzenparasiten negativ beeinflusst zu werden (Laakso \& Setälä 1998, Blomqvist et al. 2000).

Ameisen verändern die Bodenbedingungen innerhalb ihres Nestareals durch Bautätigkeiten, starke Akkumulation von Nahrungsbestandteilen und hohe Stoff- 
wechselraten schnell und nachhaltig (Pętal 1978, Woodell \& King 1991, Elmes 1991). Bodenporosität und Wasser-Infiltration nehmen zu (Lobry de Bruyn \& Conacher 1994), die Bodentemperatur, der $\mathrm{pH}-$ Wert und die Gehalte an Phosphat, Kalium und Mikronährstoffen sind im Nestboden im Vergleich zum umgebenden Boden generell erhöht, der Wassergehalt erniedrigt (Czerwiński et al. 1971, Jakubczyk et al. 1972, King 1977a, Beattie 1989, Dean et al. 1997). Schon Czerwiński et al. (1971) weisen darauf hin, dass diese Effekte sowohl von der Lebensweise der Ameisenart als auch von den jeweiligen Umweltbedingungen abhängen. King (1977a) und Dean et al. (1997) fanden im Boden aus Nesthügeln von Lasius flavus- und teilweise Lasius alienus- und Formica rufibarbis-Kolonien niedrigere Gesamt-Stickstoffgehalte als im Vergleichsboden. Pętal (1998) stellte bei vier Myrmica-Arten hingegen höhere Gehalte an verfügbarem Stickstoff fest, die allerdings von steigender Koloniegröße und Bodenfeuchtigkeit negativ beeinflusst wurden. Die Zusammensetzung der Huminstoff-Fraktionen aus Nestern von Lasius niger unterscheidet sich deutlich von der aus Myrmica-Nestern und dem umgebenden Boden (Pętal \& Kusinska 1994).

Das polnische Forscher(innen)-Team Zbigniew Czerwiński, Halina Jakubczyk und Joanna Pętal (Czerwiński et al. 1971, Jakubczyk et al. 1972, Pętal 1978, 1980) konnte im Vergleich zur Kontrollbodenprobe aus dem Nestboden verschiedener Ameisenarten z.T. extrem erhöhte Anzahlen an Bakterien und Pilzen plattieren. Dabei zeigten die drei unterschiedenen Gruppen von stärker prädatorischen $M y r$ mica-Arten mit kleineren Nestern, stärker omnivoren, teilweise hügelbauenden, monogynen Lasius niger-Kolonien und den großen, volkreichen, oft polygynen, vorwiegend im Nesthügel und unterirdisch lebenden Lasius flavus-Kolonien deutliche Unterschiede: Proben aus Nestern der Myrmica-Arten und besonders von Lasius niger enthielten sehr viel mehr ausplattierbare Bakterien und Pilze, Proben aus Lasius flavus-Nestern hingegen deutlich mehr Actinomyceten, deren Zahl bei den beiden anderen Gruppen sogar im Vergleich zum Kontrollboden niedriger war. Dauber und Wolters (2000) stellten bei Myrmica scabrinodis und den beiden Lasius-Arten erhöhte C-Mineralisationsraten fest; in Myrmica scabrinodis- und Lasius niger-Nestern war diese Steigerung der mikrobiellen Aktivität mit einer Erhöhung der mit der Biolog-Methode bestimmten funktionellen Diversität der mikrobiellen Gemeinschaft verbunden, im Nestboden von Lasius flavus scheint es sich hingegen um weniger und spezialisiertere Gruppen zu handeln.

Die klaren abiotischen und biotischen Bodenveränderungen durch die Ameisen beeinflussten in dem durchgeführten Laborversuch ohne direkte Ameiseneinwirkung deutlich sowohl das Wachstum verschiedener Pflanzenarten als auch die Nährstoffbilanzen. Die Pflanzenarten aus drei verschiedenen funktionellen Gruppen beeinflussten sich dabei gegenseitig deutlich und veränderten die Nährstoff-Dynamik, insbesondere für Stickstoff, teilweise in unterschiedlichen Interaktionen mit den indirekten bottom-up-Wirkungen der Ameisen.

Die meist endogäisch lebende Ameise Lasius flavus spielt zusammenfassend eine bedeutende Rolle als Ökosystemingenieur und moduliert folglich auch in hohem Maße bottom-up-Wirkungen für das gesamte Nahrungsnetz. 


\section{Interaktionen mit der Bodenfauna und Effekte im unterirdischen Nahrungsnetz}

\subsection{Einleitung}

Ameisen beeinflussen einerseits die Ressourcenverfügbarkeit der Pflanzen durch die deutlichen Veränderungen der Bodenchemie und -biologie (Kap. 8). Andererseits können hügelbauende Arten auch die physikalischen Bedingungen direkt auf ihrem Nesthügel drastisch verändern. Die veränderte Topographie führt zu einer stärkeren Exposition gegenüber Sonne, Wind, Erosion, Austrocknung und Verbiss durch Pflanzenfresser, wie z.B. Schafe oder Kaninchen (Woodell \& King 1991). Dazu kommen permanente Störungen durch Viehtritt und grabende Tiere: die Ameisen selbst, Mäuse, Wildschweine, Kaninchen, Rebhühner und andere (Woodell \& King 1991, Dean et al. 1997). Pflanzenökologen konnten signifikante Unterschiede zwischen der Vegetation auf den Nesthügeln von Lasius flavus und der umgebenden Vegetation in unterschiedlichen Biotopen von Trockenrasen (King 1977a) bis hin zu Salzwiesen (Woodell 1974) beobachten. Lasius flavus kann durch starke Grabtätigkeit in Verbindung mit der Anlage von Hügeln ganze Landschaften kleinräumig gestalten ("antscapes", Elmes 1991) und durch starke "patchiness" bereichern (Woodell \& King 1991, Dean et al. 1997).

Die Auswirkungen der veränderten Nährstoffgehalte des Nestbodens auf das Pflanzenwachstum testeten Dean et al. (1997), die in einem Laborversuch eine signifikante Wachstumssteigerung von Radieschen im Boden aus Ameisennesthügeln gegenüber dem Vergleichsboden feststellten. Die Autoren vermuten, dass 
trotz geringerer Gesamtstickstoff-Gehalte im Nestboden durch den erhöhten pHWert eine bessere Nährstoff-Verfügbarkeit als im sauren Heide-Boden gegeben ist.

Aus den bodenanalytischen Ergebnissen und denen eines Laborversuchs mit sterilisierter und unsterilisierter Erde aus Nestern und Kontrollboden führen Blomqvist et al. (2000) die unterschiedliche Vegetation auf den Nesthügeln auf die Lebensraum-modulierende Wirkung der Ameisen und die indirekte Wirkung über die veränderte Bodenbiota (v.a. pflanzenparasitische Nematoden) zurück.

Auch im Rahmen dieser Studien konnte eine deutliche Auswirkung der veränderten Bodenchemie auf das Pflanzenwachstum im Labor nachgewiesen werden (Kap. 8). Der im Experiment eingesetzte Boden aus den Nestern unterlag vor der Entnahme jahrelangem Ameiseneinfluss und während des Versuches keinen direkten Wirkungen der Ameisen mehr. Die Effekte von Ameisen auf den Boden und die Vegetation sind zwar vergleichsweise gut untersucht, jedoch nur selten auch im natürlichen Lebensraum durch Experimente abgesichert worden. Über die Bodenfauna in Ameisennestern ist dabei ebenfalls noch recht wenig bekannt. In dieser Untersuchung sollten deshalb einige der gut begründeten Hypothesen mit einem Mikrokosmos-Versuch im Freiland mit dominanten und auch wirtschaftlich bedeutenden Pflanzenarten getestet werden. Dazu wurden die Pflanzenarten aus verschiedenen funktionellen Gruppen, deren Wachstum schon im Laborversuch untersucht wurde, in Mikrokosmen mit gleichem Boden, die ober- und unterirdischen Zugang von Ameisen erlaubten, angezogen und entweder in Hügelnester der gelben Wiesenameise oder in umgebenden Wiesenboden gesetzt. Die Zusammensetzung der Bodenmakro- und Bodenmesofauna und die Wuchsleistungen der verschiedenen Pflanzen wurden nach einem Jahr analysiert.

\subsection{Methodik}

Im Mai und Juni 1999 wurden 60 zylindrische Mikrokosmen $(10 \mathrm{~cm}$ Durchmesser, $20 \mathrm{~cm}$ hoch, mit vier $5 \times 10 \mathrm{~cm}$ großen Seitenöffnungen) seitlich und unten mit Gaze verschlossen (Seite: $5 \mathrm{~mm}$, unten: 1,5 mm Maschenweite) und mit Erde aus dem Untersuchungsgebiet gefüllt, die zuvor durch Hitzeextraktion defauniert, getrocknet, gesiebt $(4 \mathrm{~mm})$ und gemischt wurde. In jeweils zwölf Mikrokosmen wurde eine von vier Pflanzenarten gesät: Trifolium repens, Arrhenatherum elatius, Galium mollugo und Thymus praecox (Saatgut: Conrad Appel GmbH, Darmstadt); in die verbliebenen zwölf eine Mischung aus allen vier Arten. Nachdem die Pflanzen im Freien in Witzenhausen (schneckensicher auf einem Garagendach) keimen und anwachsen konnten, wurden die Gefäße auf den Wiesen 1-3 in die Nesthügel von Lasius flavus bzw. in den umgebenden Wiesenboden eingegraben. Dazu wurden mit einem $10 \mathrm{~cm} \varnothing$ Bohrer Bodensäulen entnommen und die Mikrokosmen in die Löcher eingesetzt (Abb. 53). Die Pflanzen in den Mikrokosmen wurden mit einem Drahtkäfig oberirdisch vor Wildverbiss geschützt, die entnommene Erde gesiebt und nach Entnahme einer genauer analysierten Teilprobe (vgl. Kap. 8), rings um die Mikrokosmen verteilt, um Ameisen und anderen Bodentieren Gelegenheit zu 
geben, sich

wieder in ihr

Nest bzw.

ihren Lebens-

raum zurück-

zuziehen.

Damit alle

Pflanzen bei

Ausbringung

etwa gleich

weit entwickelt

waren, wurden

die einzelnen

Bepflanzungs-

varianten vom

26. Juli bis

zum 23. Au-

gust im wö-

chentlichen

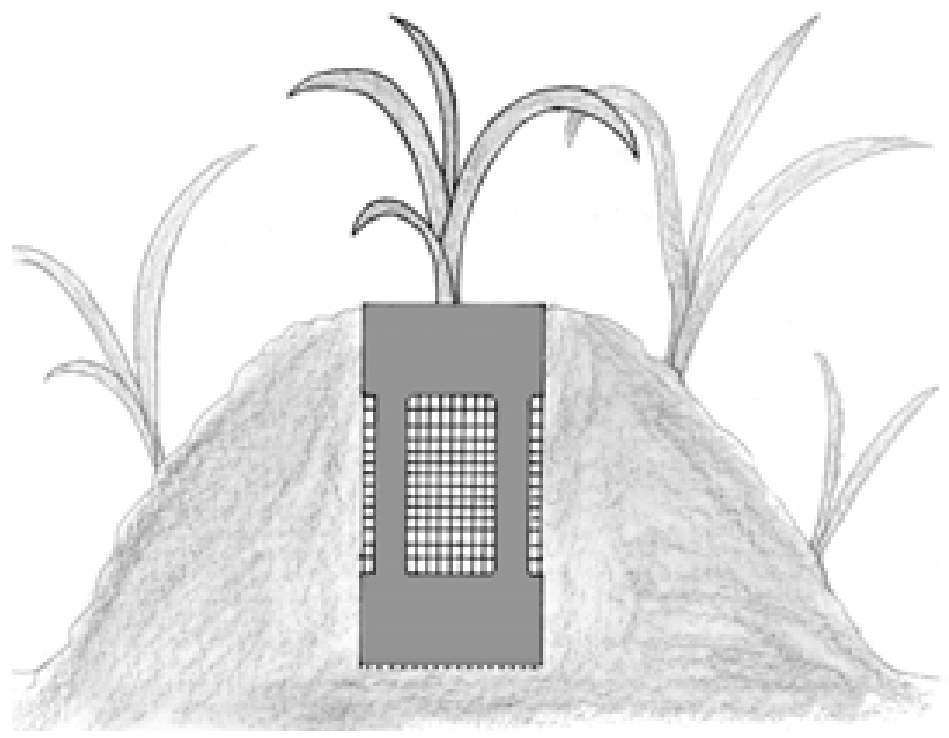

Abstand auf die Untersuchungsflächen gebracht. Die drei Wiesen wurden in jeweils zwei homogene Abschnitte mit fünf möglichst ähnlichen Nesthügeln unterteilt. Jeweils die gleiche Bepflanzungsvariante wurde in eines der Nester und $1 \mathrm{~m}$ südlich jedes Nesthügels eingesetzt, so dass die 60 Mikrokosmen auf 10 experimentelle Varianten und sechs Parallelen in Unterblöcken verteilt wurden, die in drei Blöcken - den Wiesen 1-3 - geschachtelt angelegt waren.

Die Pflanzen wurden den ersten Monat bei Bedarf gewässert und ein Jahr lang regelmäßig kontrolliert und ihre Entwicklung protokolliert. Am 7. und 8. September 2000 wurden alle Mikrokosmen entnommen (Abb. 54), die maximale Sprosslänge der Pflanzenarten gemessen und ihre Blüten gezählt und in separaten Plastiktüten ins Labor gebracht. Dort wurde die Bodensäule in vier Scheiben geschnitten: 0-5 cm, 5-6 cm, 6-12 cm und 12-18 (20) cm. Die Scheibe aus 5-6 cm Bodentiefe wurde gesiebt, der Wasser- Kohlenstoff- und Stickstoffgehalt bestimmt und die basale und substratinduzierte Atmung gemessen. Die drei anderen Horizonte wurden gemeinsam mit den oberirdischen Pflanzenteilen einer Hitzeextraktion unterzogen. Die ausgetriebenen Tiere wurden in gesättigter Kochsalzlösung aufgefangen und anschließend tiefgefroren. Bei der Mischbepflanzungsvariante wurden diese drei Tiefenzonen getrennt behandelt und es wurden zusätzlich in 11 und $17 \mathrm{~cm}$ Tiefe Bodenproben zur Analyse entnommen. Nach der Extraktion wurden die Wurzeln unter fließendem Wasser über einer Siebkaskade von der Erde getrennt und wie die oberirdischen Pflanzenteile getrocknet $\left(105^{\circ} \mathrm{C}\right)$, gewogen, gemahlen und ihre C- und N-Gehalte gemessen. 


\subsection{Ergebnisse}

Die Mikrokosmen in den Nesthügeln wurden von den Ameisen in das Nest integriert, die Bodensäulen waren von Nestkammern und Galerien durchzogen (Abb. 55) und enthielten bei der Entnahme im Mittel etwa die 100-fache Zahl an Arbeiterinnen wie die Kontrollen aus dem umgebenden Wiesenboden (Abb. 56).

Dieser enorme Unterschied in der Ameisenaktivität hatte eine hochsignifikante

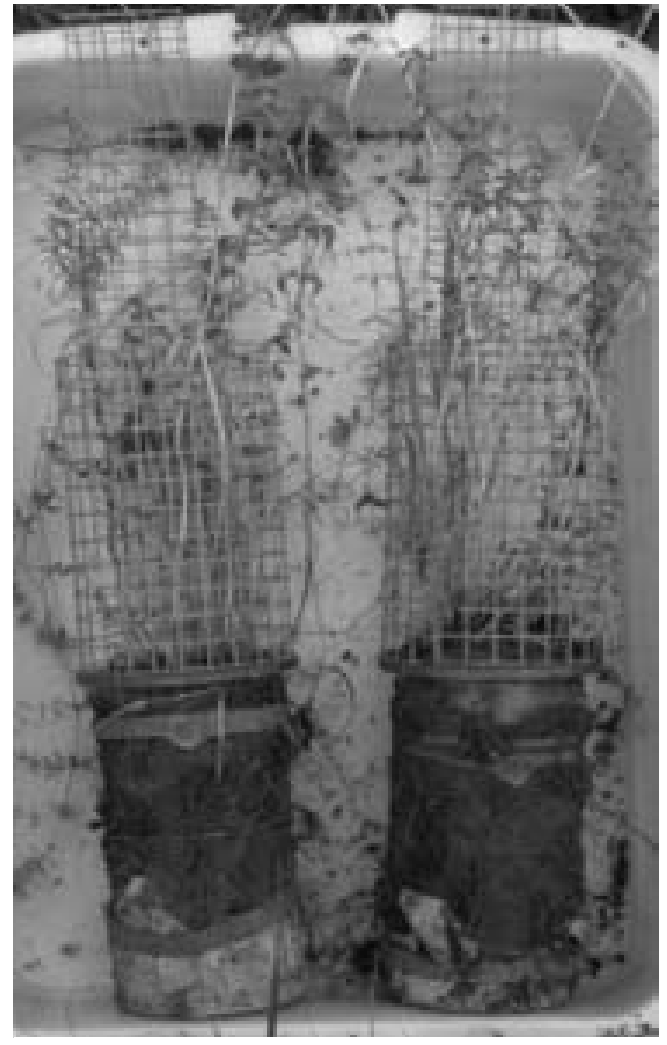

Abb. 54: Zwei Freiland-Mikrokosmen von der Wiese (links) und aus dem Nesthügel von Lasius flavus (rechts) mit Misch-Bepflanzung direkt nach Entnahme aus dem Boden im September 2000.

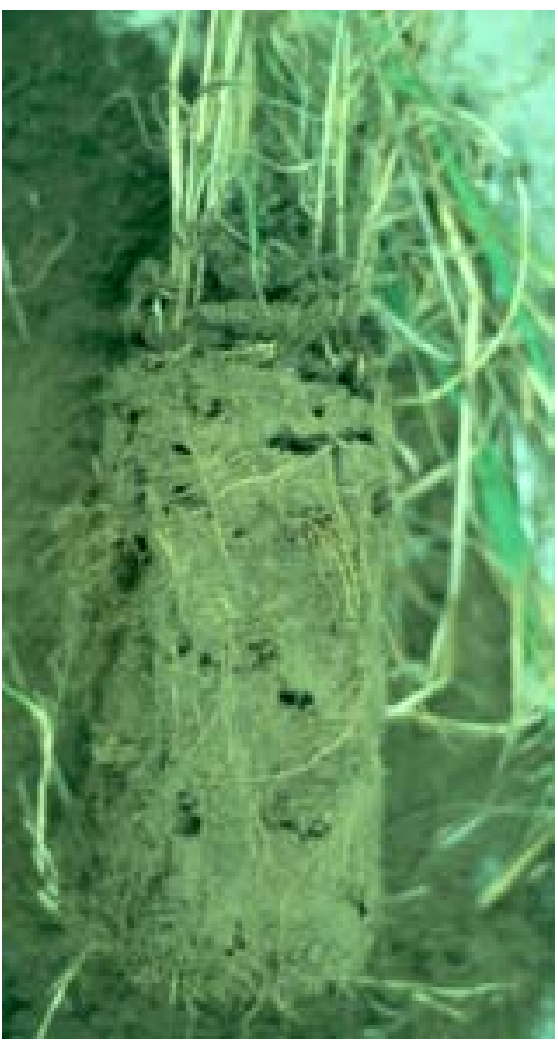

Abb. 55: Bodensäule eines Mikrokosmos aus dem Nesthügel von Lasius flavus mit Glatthafer-Bepflanzung.

Erhöhung der mikrobiellen Biomasse im Boden der Gefäße innerhalb der Ameisennester zur Folge (Tab. 21). Ebenso war das Verhältnis von mikrobiellem zu organischem Kohlenstoff deutlich höher. Die Gesamt-Stickstoffgehalte im Boden waren deutlich gegenüber dem Ausgangsmaterial erhöht, aber in den Ameisennestern signifikant geringer als im Vergleichsboden, die Unterschiede zwischen den Blöcken waren noch deutlicher. 

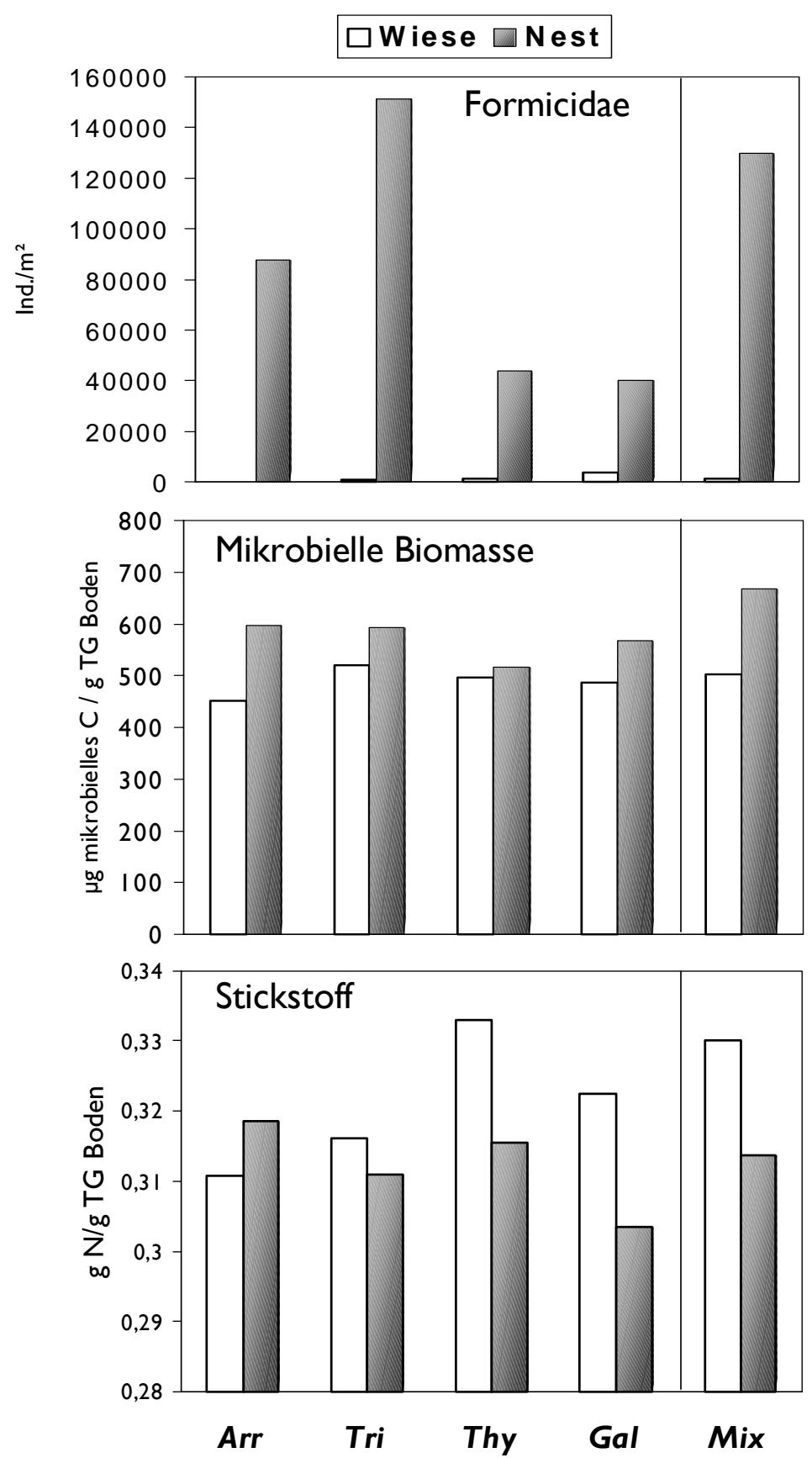

Abb. 56: Mittlere Dichte der Ameisen, mikrobielle Biomasse und Gesamt-Stickstoffgehalte des Bodens in den Mikrokosmen, die ein Jahr in Nestern von Lasius flavus oder im umgebenden Wiesenboden inkubiert wurden. Arr = Arrhenatherum elatius, Tri= Trifolium repens, Thy= Thymus praecox, Gal= Galium mollugo, Mix= Mischung aller vier Pflanzenarten . 

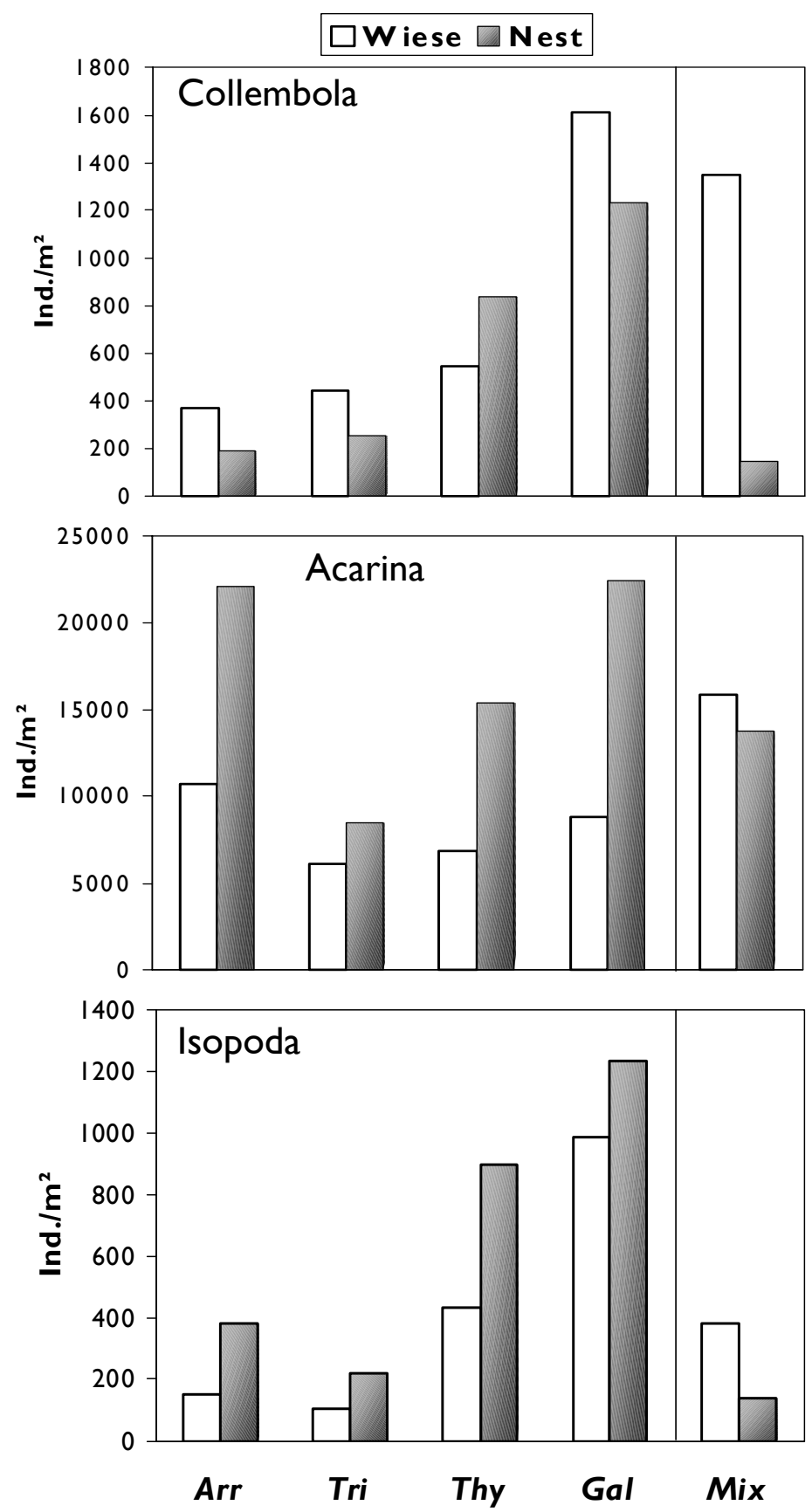

Abb. 57: Mittlere Dichte der Springschwänze, Milben und Asseln in Mikrokosmen, die ein Jahr in Nestern von Lasius flavus oder im umgebenden Wiesenboden inkubiert wurden. Arr= Arrhenatherum elatius, $T r i=$ Trifolium repens, Thy $=$ Thymus praecox, Gal= Galium mollugo, Mix = Mischung aller vier Pflanzenarten. 
Die Dichte der Collembola war tendenziell in den mit Wiesen-Labkraut (Galium mollugo) bepflanzten Varianten außerhalb der Nesthügel am höchsten; nur in der Thymian-Variante waren in den Mikrokosmen innerhalb der Nester mehr Tiere als im Wiesenboden. Doch die Varianzen innerhalb der gleichen Behandlungsstufen waren sehr hoch, so dass keiner dieser Unterschiede auf signifikanten Effekten beruhte (Tab. 21), die Unterschiede zwischen den drei Wiesen waren in jedem Falle deutlicher.

Die Milben insgesamt hingegen waren in den Gefäßen innerhalb der Nester der Gelben Wiesenameise signifikant häufiger (Abb. 57). Hornmilben (Oribatida) hatten auf zwei der drei Blöcke innerhalb der Nesthügel deutlich niedrigere Dichten. Insbesondere zoophage Milbengruppen bevorzugten solche Mikrokosmen, die stark von Ameisen besiedelt waren. Auch die saprophagen Isopoda hatten in den Ein-Arten-Gefäßen in den Ameisennestern höhere Dichten, besonders in solchen mit Thymian und Galium. Für die Asseln war neben dem Unterschied zwischen den Pflanzen auch der Einfluss der Wiese signifikant. Sowohl die Mesofauna als auch die Asseln als Vertreter der saprophagen Makrofauna hatten auf Wiese 3 höhere Dichten als auf Wiese 2; auf Wiese 1 hingegen hatten Isopoda ihr Dichteminimum und Collembola ihr Dichtemaximum.

Die zoophage Makrofauna reagierte ganz anders auf die Unterschiede in der Ameisendichte als die prädatorischen Milben: sowohl Spinnen als auch Steinläufer haben in den Nestern die Mikrokosmen in signifikant niedrigeren Dichten besiedelt als auf der Wiese (Abb. 58, Tab. 21). Die phytophagen Blattläuse besiedelten ihre beiden in diesem Versuch bevorzugten Futterpflanzen, Glatthafer und Wiesenlabkraut, in höheren Dichten, wenn sie in Nesthügeln der hypogäischen Ameisen wuchsen. Die Pflanzenmischung hingegen hatte in den Nestern geringere Blattlausdichten als auf der Wiese.

Die Pflanzenarten reagierten sehr unterschiedlich auf die anderen Wuchsbedingungen in den Nestern im Vergleich zum Wiesenboden: Galium hatte auf den Nesthügeln sowohl einzeln als auch in Mischung wachsend höhere oberirdische Biomassen, Trifolium nur in Monokultur - in Konkurrenz zu den anderen Arten wurde der Klee auf den Nesthügeln komplett verdrängt (Abb. 59). Thymus und Arrhenatherum hatten auf den Nesthügeln sowohl einzeln als auch in Mischung niedrigere Sprosstrockengewichte. Blütenzahl und Sprosshöhe unterschieden sich zum Teil sehr deutlich, ließen aber keine Unterschiede zwischen den Nesthügeln und der umgebenden Wiese erkennen. Unterirdisch hatten die drei krautartigen Pflanzenarten signifikant höhere Wurzelmassen in den Mikrokosmen innerhalb der Nesthügel, nur das Gras hatte auf der Wiese tendenziell etwas schwerere Wurzeln. Die Stickstoffmengen in den Wurzeln waren bei allen vier Arten in den Ameisennestern signifikant höher (Abb. 59, Tab. 21). 

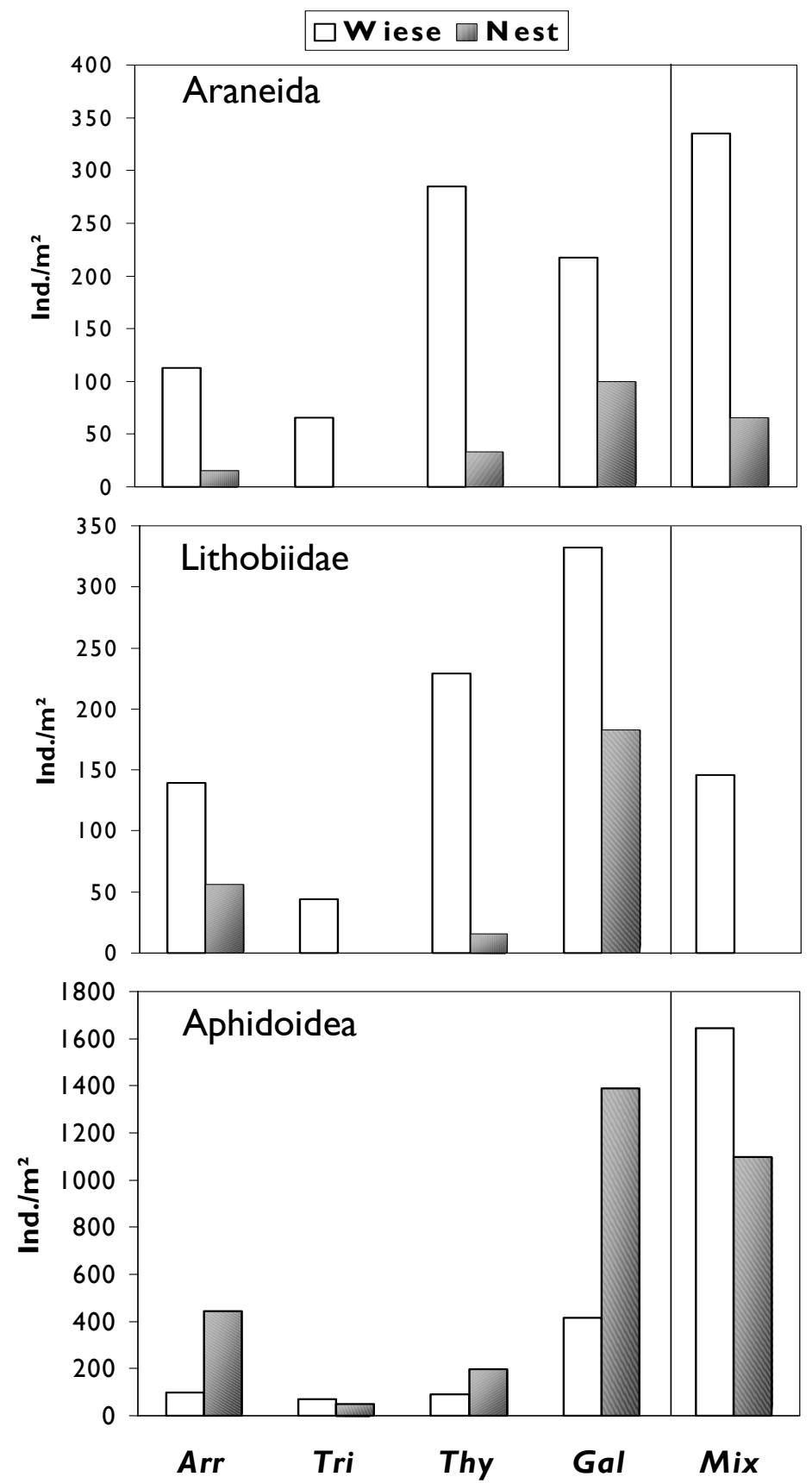

Abb. 58: Mittlere Dichte der Webspinnen, Steinläufer und Blattläuse in Mikrokosmen, die ein Jahr in Nestern von Lasius flavus oder im umgebenden Wiesenboden inkubiert wurden. Arr= Arrhenatherum elatius, Tri $=$ Trifolium repens, Thy $=$ Thymus praecox, Gal $=$ Galium mollugo, Mix = Mischung aller vier Pflanzenarten. 

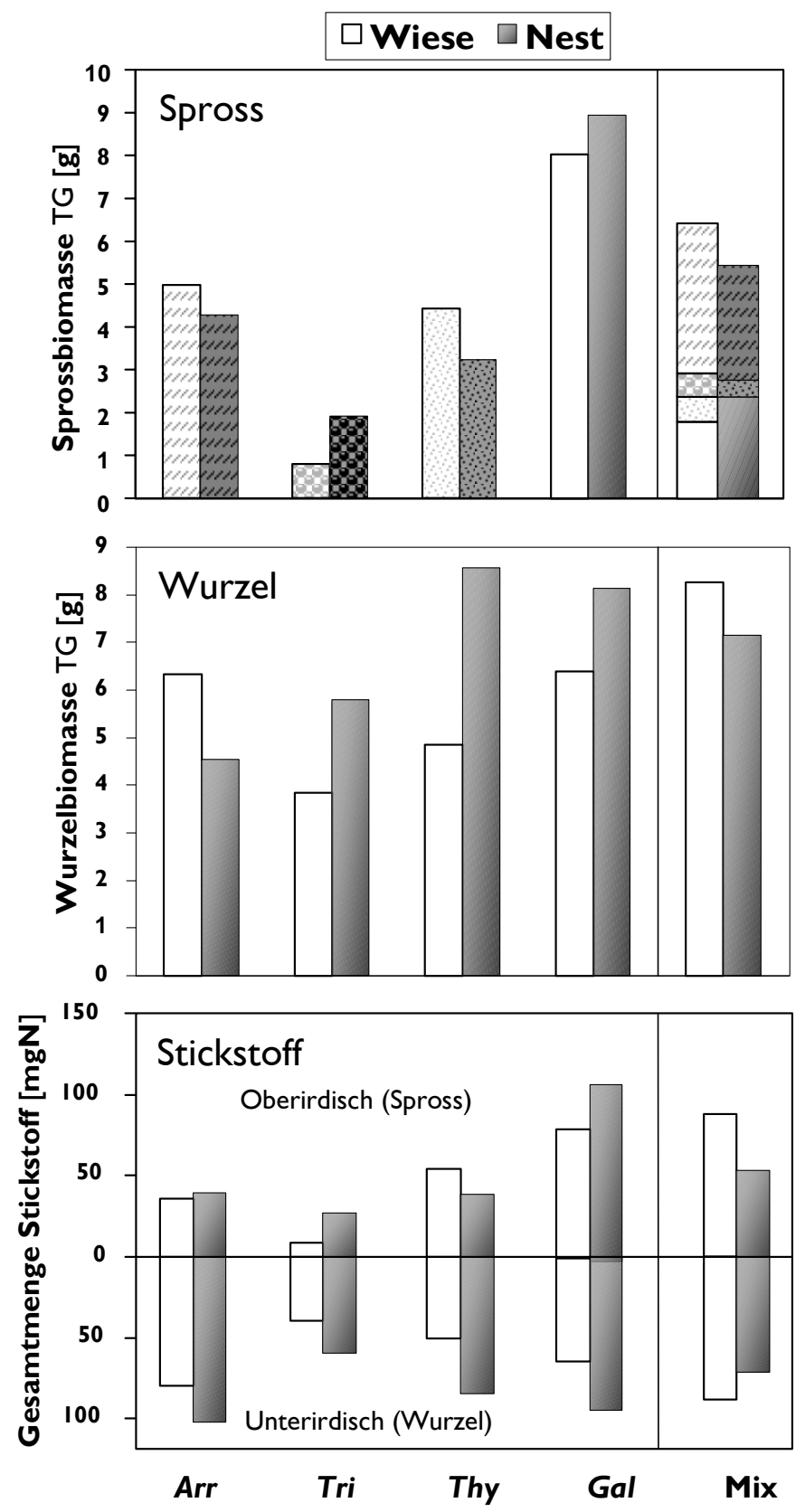

Abb. 59: Mittlere Biomassen der ober- und unterirdischen Pflanzenteile und die darin enthaltenen Gesamtmengen an Stickstoff in Mikrokosmen, die ein Jahr in Nestern von Lasius flavus oder im umgebenden Wiesenboden inkubiert wurden. Arr = Arrhenatherum elatius, Tri $=$ Trifolium repens, Thy $=$ Thymus praecox, Gal $=$ Galium mollugo, Mix $=$ Mischung aller vier Pflanzenarten. 
Tabelle 21: F-Werte (mit Freiheitsgraden [Effekt; Febler] als Index) und Wabrscheinlichkeiten für $\alpha$-Febler (p) der Effekte von Inkubation im Ameisennest bzw. Wiesenboden der Bepflanzungsvariante, deren Interaktionen und der Blöcke aus zweifaketoriellen Varianzanalysen im Blockedesign mit den Abundanzen von Ameisen, Mikrophytophagen, Saprophagen, Zoophagen und Phytophagen sowie den Biomassen von Pflanzenteilen und Mikroflora und den Stickstoffgehalte von Pflanzenteilen

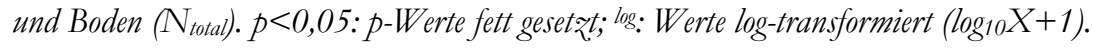

\begin{tabular}{|c|c|c|c|c|c|c|c|c|}
\hline & \multicolumn{2}{|c|}{ Ameisennest } & \multicolumn{2}{|c|}{ Pflanzenart } & \multicolumn{2}{|c|}{$A \times P$} & \multicolumn{2}{|c|}{ Block } \\
\hline & $\mathrm{F}_{1 ; 48}$ & $\mathrm{p}$ & $\mathrm{F}_{4 ; 48}$ & $\mathrm{p}$ & $\mathrm{F}_{4 ; 48}$ & $\mathrm{p}$ & $\mathrm{F}_{2 ; 48}$ & $\mathrm{p}$ \\
\hline Formicidae ${ }^{\log }$ & 107,36 & $<0,0001$ & 0,73 & 0,5730 & 2,05 & 0,1024 & 1,38 & 0,2616 \\
\hline $\mathrm{C}_{\text {mic }}$ & 17,80 & 0,0001 & 1,44 & 0,2351 & 1,30 & 0,2821 & 2,27 & 0,1146 \\
\hline $\mathrm{N}_{\text {total }}$ & 5,54 & 0,0227 & 1,20 & 0,3237 & 1,40 & 0,2471 & 12,26 & $<0,0001$ \\
\hline Collembola & 0,01 & 0,9215 & 0,56 & 0,6913 & 0,72 & 0,5794 & 1,03 & 0,3661 \\
\hline Acarina & 6,45 & 0,0144 & 0,88 & 0,4806 & 0,60 & 0,6623 & 0,40 & 0,6744 \\
\hline Isopoda & 1,79 & 0,1878 & 2,69 & 0,0421 & 1,10 & 0,3693 & 4,39 & 0,0177 \\
\hline Araneida & 13,12 & 0,0007 & 2,19 & 0,0840 & 1,07 & 0,3816 & 2,99 & 0,0595 \\
\hline Lithobiidae & 9,11 & 0,0041 & 2,67 & 0,0432 & 0,64 & 0,6356 & 0,42 & 0,6603 \\
\hline Aphidoidealog & 1,92 & 0,1723 & 8,05 & $<0,0001$ & 1,03 & 0,3996 & 0,08 & 0,9243 \\
\hline Spross & 0,17 & 0,6853 & 8,23 & $<0,0001$ & 0,51 & 0,7285 & 0,12 & 0,8872 \\
\hline Wurzel & 5,51 & 0,0230 & 6,56 & 0,0003 & 1,13 & 0,3544 & 1,25 & 0,2945 \\
\hline Spross- $\mathrm{N}_{\text {total }}$ & 0,00 & 0,9878 & 7,82 & $<0,0001$ & 1,45 & 0,2326 & 0,14 & 0,8661 \\
\hline Wurzel- $\mathrm{N}_{\text {total }}$ & 5,77 & 0,0202 & 3,37 & 0,0165 & 1,48 & 0,2243 & 0,96 & 0,3904 \\
\hline
\end{tabular}

\subsection{Diskussion}

Ameisen beeinflussen Pflanzen nicht nur als Ökosystemingenieure durch indirekte bottom-up-Effekte sondern auch trophisch direkt durch Blatt- oder Samenfraß und indirekt durch Trophobiose mit Pflanzensaftsaugern und die Vertilgung anderer Pflanzenfresser. Direkte Phytophagie ist bei Ameisen jedoch eher selten, oft sind die direkten Einwirkungen auf Pflanzen auf Samenverbreitung oder Blütenbestäubung beschränkt (Huxley \& Cutler 1991).

Bestimmte Pflanzenarten wie z.B. das Quendel-Sandkraut (Arenaria serpyllifolia) oder einige Thymiane (Thymus spec.) zeigten eine auffällige Affinität zu Ameisenhaufen (King 1977a). Die Nester werden entweder durch Ausläufer aus der direkten Nestumgebung oder durch schnelle, kurzlebige Arten mit hoher Samenproduktion besiedelt (King 1977b). King (1977c) schloss weiterhin aus Versuchen auf eine relativ gute Keimfähigkeit auf der Spitze der Nesthügel, kombiniert mit einer hohen, dichte-unabhängigen Mortalität, was die Neuansiedlung von r-Strategen begünstige.

Auch bei Myrmica-Arten, die allenfalls kleine Erhebungen anlegen, konnte im Nestbereich eine signifikant verschiedene Vegetation gefunden werden, die durch das gezielte Aufsuchen von Stellen mit niedriger Pflanzendecke durch die Ameisen 
bei der Koloniegründung oder -umsiedlung erklärt werden kann (Elmes \& Wardlaw 1982).

Bei meinen Untersuchungen konnte ich auf einigen Nesthügeln deutlich veränderte Vegetation feststellen, auf anderen hingegen eher nur eine stärkere Dominanz der beiden auch in der Wiese häufigsten Pflanzenarten, Arrhenatherum elatius und Galium album. Auf Wiese 1 waren einige Hügel von Lasius flavus im Übergangsbereich zum Halbtrockenrasen komplett mit einer dichten Decke von Thymian bedeckt. Einige stärker beschattete Nesthügel waren verstärkt von einer dichten Moosschicht bedeckt und alle Erdhügel hatten keine verfilzte Streuauflage, die auf der gesamten übrigen Fläche kennzeichnend ist, und vielen Tieren wichtige Strukturen und mikroklimatisch besondere Kleinsträume bietet. So ist beispielsweise die größere Spinnendichte auf der Glatthaferwiese, die v.a. von kleinen Jung- und Zwergspinnen ausgemacht wird, vermutlich in der geeigneteren Habitatstruktur begründet, wie auch der Vergleich verschiedener Lebensräume (Kap. 4) und die Entwicklung im Verlauf des Klimaexperiments (Kap. 5) deutlich machen. Gleichzeitig haben Spinnen und Steinläufer - nach den Ameisen die beiden wichtigsten räuberischen Taxa - auch direkte negative Interaktionen durch Störung und gegenseitigen Fraß (vgl. Kap. 7), da Ameisen sowohl in der Vegetation, der Streu als auch im Boden aktiv sind. Direkt auf ihren Nesthügeln ist Lasius flavus auch zumindest insofern aktiv, als dass sie durch ihre starke Grabaktivität die Oberfläche ständig stört und umgestaltet.

Für die hier ausgewählten Pflanzenarten sind positive, neutrale und negative Einflüsse von hügelbauenden Ameisen beschrieben worden: Thymian ist auf den Nesthügeln häufiger, Klee seltener, Glatthafer und Wiesenlabkraut können durch ihre Ausläuferbildung gut mit den veränderten Bedingungen auf und in den Nestern zurechtkommen, zeigten aber keine deutlichen Präferenzen (King 1977a). In diesem Versuch reagierten jedoch gerade Klee und Thymian umgekehrt auf die veränderten Ameisendichten innerhalb und abiotischen Bedingungen außerhalb der Mikrokosmen. Trifolium repens hatte auf allen Wiesen schlechte Bedingungen, da seine Laubblätter auffallend oft von Schnecken abgefressen wurden. Die Spross- und Wurzel-Biomassen waren dennoch auf den Nesthügeln höher, wenn Klee allein im Gefäß wachsen konnte. In Konkurrenz mit den anderen Arten waren vom Klee nur auf den Wiesen-Mikrokosmen noch oberirdische Pflanzenteile vorhanden. Auch im Laborversuch konnte Klee mit vergleichsweise geringen Wurzelbiomassen in Boden sowohl vom Nest als auch von der Wiese hohe Biomassen aufbauen (Kap. 8). Ein Konkurrenzeffekt war zwar auch hier deutlich erkennbar, er beeinflusste aber nicht die positive Wirkung des Nestbodens. Bei besserer Stickstoffversorgung machen sich die hohen Energiekosten der Stickstoff-fixierenden Symbionten bei den Leguminosen nachteilig bemerkbar; so besiedelt Klee in erster Linie solche Boden-Flecken, die geringe Stickstoffgehalte haben, solange bis dieser auch dort durch die eigenen Symbionten erhöht ist und der Klee mit seinen Ausläufern quasi auf andere Flecken ,auswandern“ muss (Begon et al. 1996). Thymian hatte wie der Weißklee trotz der großen Störungen durch die Kammern und Gänge grabenden Ameisen in den Nestern ein höheres 
Wurzeltrockengewicht. Die artspezifischen Wurzelmassen ließen sich in den MixVarianten wegen der hohen Verfilzung nicht bestimmen.

Wiesenlabkraut konnte in den Ameisennestern trotz etwa dreimal höherer Blattlausbesiedlung immer noch etwas höhere Spross- und Wurzelbiomassen ausbilden. Glatthafer hatte zwar als einzige Art in den Nestern auch unterirdisch geringere Biomassen, doch wegen der deutlich höheren Stickstoffgehalte war in den Glatthaferwurzeln eine größere Gesamtmenge an Stickstoff enthalten, wenn diese in den Nesthügeln wuchsen.

Insgesamt überwiegen für die Ameise Lasius flavus offensichtlich die bottomup-Wirkungen auf Ökosystemprozesse durch Veränderung der Ressourcenverfügbarkeit gegenüber den trophischen Interaktionen. Mikrobielle Biomasse und Nährstoffgehalte sind in den Nesthügeln signifikant erhöht, deren Wirkung auf das Pflanzenwachstum ist ohne Ameisenanwesenheit deutlicher. 


\section{Diskussion \& Schlussfolgerungen}

„Geh hin zur Ameise, ..., sieh ihr Tun an, damit Du schlau werdest“ (Salomo, 965 - 928 v. Chr.) - offensichtlich wurden die Ameisen schon vor 3000 Jahren als Modellorganismen für das tiefere Verständnis ökologischer Zusammenhänge erkannt. Am Beispiel der Ameisen als Schlüsseltiergruppe im Grasland habe ich das Zusammenspiel trophischer und nicht-trophischer Interaktionen untersucht. Diese Studie erstreckte sich von der Erfassung der Ameisenfauna in Bezug zur übrigen Tiergemeinschaft in einem Mosaik verschiedener biotischer und abiotischer Umwelteinflüsse über die experimentellen Nachweise der Bedürfnisse der Tiere an Mikroklima und Nahrung bis hin zu ihren synchronen Auswirkungen als hochabundante Prädatoren und Gestalter des ökologischen Systems Boden.

Die gleichzeitige Wirkung über Feinddruck auf andere Tieren und Modulation der Produktivität sollte eine Schlüsselstellung der Ameisen im System Halbtrockenrasen bzw. Glatthaferwiese bedingen. Zur Überprüfung der Rolle verschiedener Ameisen im Ökosystem wurden die Einflüsse ihrer gestaltenden Tätigkeiten und trophischen Interaktionen auf das Wachstum verschiedener Pflanzenarten getestet.

In dieser Studie wurden die Analysen in folgenden Schritten zusammengefasst und diskutiert:

1. Ökologische Bedingungen für verschiedene Ameisenarten auf Populations- und Gemeinschaftsebene.

2. Einfluss der Ameisen auf die Ausformung eines Nahrungsnetzes.

3. Beteiligung an Ökosystemprozessen und den sie steuernden Vorgängen. 
Den Fragestellungen, die auf diesen drei Ebenen bearbeitet wurden, lagen zwei zentrale Hypothesen zugrunde, zum einen dass die Ameisen selbst vor allem von Klima und Konkurrenz abhängen, zum anderen dass sie über zwei grundsätzlich verschiedene Wirkpfade - als Beutegreifer und Ökosystemingenieure - ihre Umwelt verändern und gestalten. Die Schlussfolgerungen aus den einzelnen Untersuchungsteilen können aber nur in gemeinsamer Betrachtung der drei Ebenen gezogen werden. Komplexe Systeme bedürfen komplexen Analysen - dennoch soll versucht werden, die wichtigsten Zusammenhänge in einem Interaktionsschema zusammenzufassen, das um die Ameisen herum aufgebaut wurde.

Bei der Untersuchung der Ameisenbesiedlung auf einer Fläche an der Schnittstelle verschiedener Lebensräume (Wiese 1, Kap. 4) wurden in allen Habitattypen vom Trockenrasen über Streuobstwiesen und Buschwerk bis in den Mischwald Ameisen gefunden, es gab kein Stück Boden, das frei von Ameisen war. Dennoch hatten die Ameisen sowohl die größten Biomassen als auch die höchste Diversität in den wiesenartigen Lebensräumen. Nur aufgrund der Verteilungsanalysen konnten keine Anhaltspunkte für Konkurrenz weder mit der zweiten wichtigen Zoophagengruppe, den Spinnen, noch innerhalb der Ameisen gefunden werden. Auch die wichtigsten Phytophagen, die Homoptera, korrelierten positiv mit Spinnen und Ameisen. Das lässt entweder den Schluss auf eine RessourcenLimitierung (bottom-up) der Zoophagen oder einfach gleiche Habitatansprüche zu. Interspezifische Konkurrenz tritt bei Ameisen vorwiegend zwischen ökologisch ähnlichen Arten auf (z.B. Elmes \& Wardlaw 1982, Seifert 1996); in einer aktuellen experimentellen Studie konnte für die in einem Hierarchiemodell dominante australische Art Iridomyrmex purpureus auch nur auf ökologisch ähnliche Arten der gleichen Gattung ein starker Einfluss nachgewiesen werden (Gibb \& Hochuli 2004). Auf den von mir untersuchten Flächen kamen solche Schwesterarten-Paarungen nicht vor, zwischen unterschiedlicheren Ameisenarten konnte ich eher Konflikt-Vermeidungs-Strategien beobachten, da sich die Arbeiterinnen überwiegend aus dem Weg gingen.

Für die Ameisen lassen sich Abhängigkeiten von der Bodentemperatur erkennen, die durch den experimentellen Nachweis der Bedeutung der Sonneneinstrahlung (Kap. 5) gestützt werden. Habitatstrukturen waren dabei weniger bedeutend, zumal auf den untersuchten Wiesen wenige flache Steine lagen, die von vielen Ameisenarten als Ort für die Nestanlage bevorzugt werden. Von den Arten mit hohen Individuendichten und Biomassen sind besonders die Lasius-Arten durch die Anlage eines Nesthügels von der Habitatstruktur auf der Mikroskala relativ unabhängig. Nur weniger abundante Arten wie Stenamma debile zeigten eine Abhängigkeit von z.B. der Streuauflage. Neben der starken Abhängigkeit von klimatischen Bedingungen konnten im Laborversuch zumindest für Lasius niger auch deutliche trophische Bedürfnisse festgestellt werden (Kap. 6), so dass ein funktioneller Zusammenhang zwischen den hohen Dichten der Homoptera und denen der Ameisen sehr wahrscheinlich ist. Ohne ausreichendes Angebot an Honigtau könnten die individuenreichen Ameisenvölker ihre energieaufwendige Lebenswei- 
se nicht verwirklichen. Erst bei genügend Sonneneinstrahlung und Kohlenhydratquellen können die Ameisen auch als Zoophage einen Einfluss ausüben.

Für die Spinnen hat sich auch in diesen Untersuchungen mehrfach die deutliche Abhängigkeit von der Habitatstruktur bestätigt: sowohl am Boden (Kap. 4 \& 5) als auch auf Büschen und Bäumen (Kap. 7) hatten strukturreiche Elemente die höchsten Spinnendichten, was sehr gut mit Ergebnissen aus der Waldforschung übereinstimmt (Halaj et al. 2000, Platner et al. 2001, Schaefer 2002, Scheu et al. 2003). Positive Korrelationen zwischen Ameisen und Spinnen lassen sich oft durch Überschneidungen der jeweiligen Habitatansprüche erklären. Viele vagante Spinnen bevorzugen wie die Ameisen offene, sonnige Bereiche; Netzspinnen finden in diverser Vegetation mehr Netzbauplätze, Ameisen finden dort mehr Phytophage. Das könnte auch die widersprüchlichen Ergebnisse verschiedener deskriptiver Arbeiten erklären: Floren \& Otto (2002) konnten aus von Formica polyctena belaufenen Eichenkronen durch Benebelung mehr Spinnenarten und -individuen herausholen als aus Vergleichsbäumen ohne Ameisen; andere Arbeiten hingegen fanden im Einzugsbereich von Waldameisennestern weniger Spinnen und andere Zoophage und konnten im Labor die negativen Interaktionen zwischen Ameisen und Spinnen bestätigen (Cherix \& Bourne 1980, Gridina 1990, 1994). Die Kronen der ameisenbelaufenen Eichen könnten auch für Spinnen ein besseres Mikroklima oder auch Nahrungsangebot geboten haben; bei experimentellem Ausschluß von Ameisen in Baumkronen konnten Halaji et al. (1997) negative Wirkungen auf vagante Spinnen feststellen. Auch in meinen Untersuchungen konnte ich experimentell eine direkte negative Wirkung von Ameisen insbesondere auf Baldachinnetzspinnen sowohl am Boden als auch in der höheren Vegetation feststellen. Krabbenspinnen hatten auf Kiefernzweigen ohne Ameisenzugang geringere Individuendichten, allerdings auch ein geringeres Nahrungsangebot und mehr Konkurrenten (Kap. 7).

Die geringe top-down-Wirkung der Ameisen und vaganten Spinnen im Ausschlussversuch könnte zum Teil auf der negativen Wechselwirkung mit netzbauenden Spinnen beruhen. Für Argiope bruennichi konnte kürzlich eine deutliche negative Wirkung auf Zikaden nachgewiesen werden (Taraschewski et al. 2005, Sanders, Grützner, Nickel \& Platner, unveröffentlicht); im naturnahen Graslandökosystem könnte Intragilden-Interaktion die top-down Wirkungen reduzieren. 


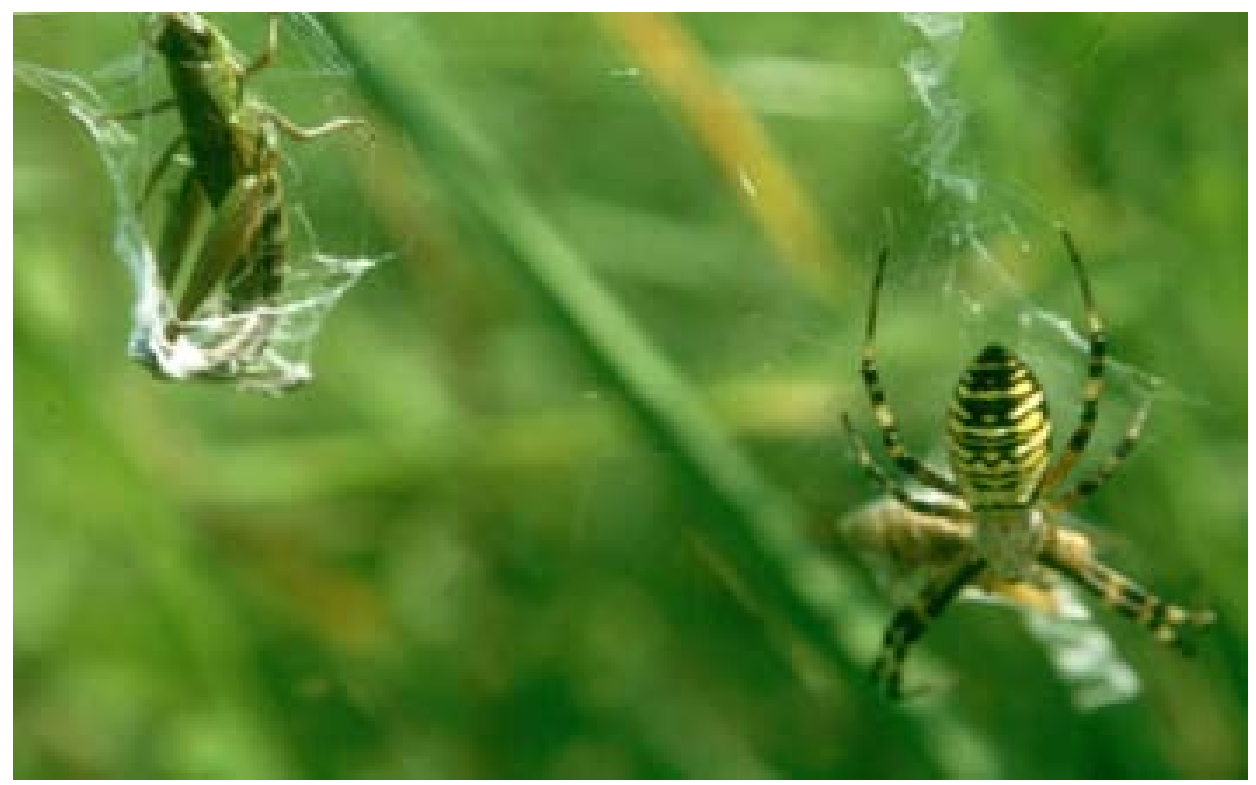

Abb. 60: Trophische Interaktionen im „Nahrungsnetz".

Ameisen fangen regelmäßig Spinnen und tragen sie in ihr Nest ein, doch stellen Spinnen meist nur einen kleinen Teil der Beute dar (Cherix \& Bourne 1990, Halaj et al. 1997); die Analyse der stabilen Isotope stützt diese Annahme auch für den Halbtrockenrasen, da die Ameisen bei stärkerem Verzehr von Spinnen eine höhere trophische Ebene erreichen sollten. Nur die größeren Arbeiterinnen der Gattung Formica und die rein zoophagen der Gattung Ponera sind mit den vaganten Spinnen auf der gleichen trophischen Ebene. Jungspinnen kommen sowohl für die Ameisen als auch für die größeren Spinnen als wichtiger Nahrungsbestandteil in Betracht. Eine Bestimmung der Nahrungsressourcen nur durch die Analyse stabiler Isotope ist aber gerade für Ameisen schwierig, da die meisten Arten erhebliche Mengen Honigtau konsumieren, der auch Aminosäuren enthält und die Ameisen so in der Summe auf ein mittleres trophisches Niveau kommen. Kirchner (1990) beschreibt den eher seltenen Fang von Ameisenarbeiterinnen durch Theridiidae und Agelenidae und den von Geschlechtstieren durch Linyphiidae und Araneidae. Vagante Spinnen könnten die wehrhaften Ameisen als Beute eher meiden - am Honigtopf konnte ich einmal beobachten, wie ein Alopecosa-Weibchen eine Lasius alienus-Arbeiterin fing, etwa zwei Zentimeter weit trug und dann wieder fallen ließ, woraufhin sich beide Prädatoren in entgegengesetzte Richtungen entfernten. Von der trophischen Ebene der Spinnen her kommen eher Lasius und Myrmica als gelegentliche Beute der größeren Spinnen in Frage - im Ausschlussversuch hatten die Spinnen aber nur eine schwache, nicht signifikante Wirkung auf die Dichte der Ameisen.

Bei der experimentellen Manipulation des Mikroklimas reagierten die Spinnen wie die Ameisen negativ auf die Beschattung, allerdings wurde diese Tendenz für 
die Spinnen erst im Verlauf des Experiments signifikant, was eine stärkere indirekte Wirkung der Vegetation vermuten lässt. Die Weberknechte hatten anfangs ihre geringsten Dichten in den Varianten mit den höchsten Ameisendichten; nach zwei Jahren stellte sich für alle Zoophagen ein deutlicher Einfluss der Vegetation bzw. Habitatstruktur heraus. Für alle häufigeren saprophagen Tiergruppen war der Faktor Niederschlag signifikant. Regenwürmer hatten auf ausreichend feuchten und besonnten Parzellen zusammen mit den Ameisen hohe Dichten, zumindest im Frühjahr waren sie auch nicht selten innerhalb der Nesthügel von Lasius flavus zu finden, Schleim könnte sie dabei vor Prädation schützen (Laakso \& Setälä 1997). Wurde jedoch das Mikroklima verändert, reagierten die beiden Gruppen der Bodenwühler mit den größten Biomassen entgegengesetzt: bei Beschattung gab es deutlich weniger Ameisen, bei Austrocknung deutlich weniger Regenwürmer. Ein Klimawandel mit wärmeren, trockeneren Sommern könnte zu einer Verschiebung in Richtung tropenähnliche Verhältnisse führen, wo die Lumbricidae keine Rolle mehr spielen, Ameisen dafür umso mehr. Eine Sukzession mit starker Beschattung hätte einen schnellen Wandel in waldähnliche Bedingungen mit vielen Regenwürmern und wenig Ameisen zur Folge. So können klimatische Veränderungen die steuernde Wirkung auf Ökosystemprozesse deutlich verändern. Doch zur Überprüfung und Spezifizierung solcher Hypothesen bedarf es weiterer Experimente, die am besten Gradientenuntersuchungen miteinschließen (Dunne et al. 2004).

Klimatische Faktoren wirken aber meist auf einer großen Skala, Interaktionen im Nahrungsnetz hingegen auf einer kleinen (Bardgett 2002). Auf einer mittleren Skala wirken die größten Ökosystemingenieure, die Rinder, die durch einen anderen Ingenieur, den Menschen, durch Dezimierung ihrer Feinde und Schaffung größerer Weideflächen von ihren top-down und bottom-up Kontrollen befreit wurden (Wardle \& Bardgett 2004). In frühen Sukzessionsstadien können die groBen Pflanzenfresser durch Verbiss des Aufwuchses auch für viele Ameisenarten die Lebensgrundlage erhalten. Doch Rinderbeweidung wirkt nicht nur oberirdisch, sondern durch Veränderung der pflanzlichen Artenzusammensetzung, Streuqualität und des Wurzelwachstums auch unterirdisch (Bardgett et al. 1998), dabei kann die mikrobielle Biomasse deutlich reduziert werden (Sankaran \& Augustine 2004). Auch bei Besonnung und Austrocknung trat eine Reduktion der Mikroflora und der Saprophagen ein, die Beweidung könnte also auch indirekt über die reduzierte Abdeckung der Bodenoberfläche durch die dichte, verfilzte Vegetation wirken. 


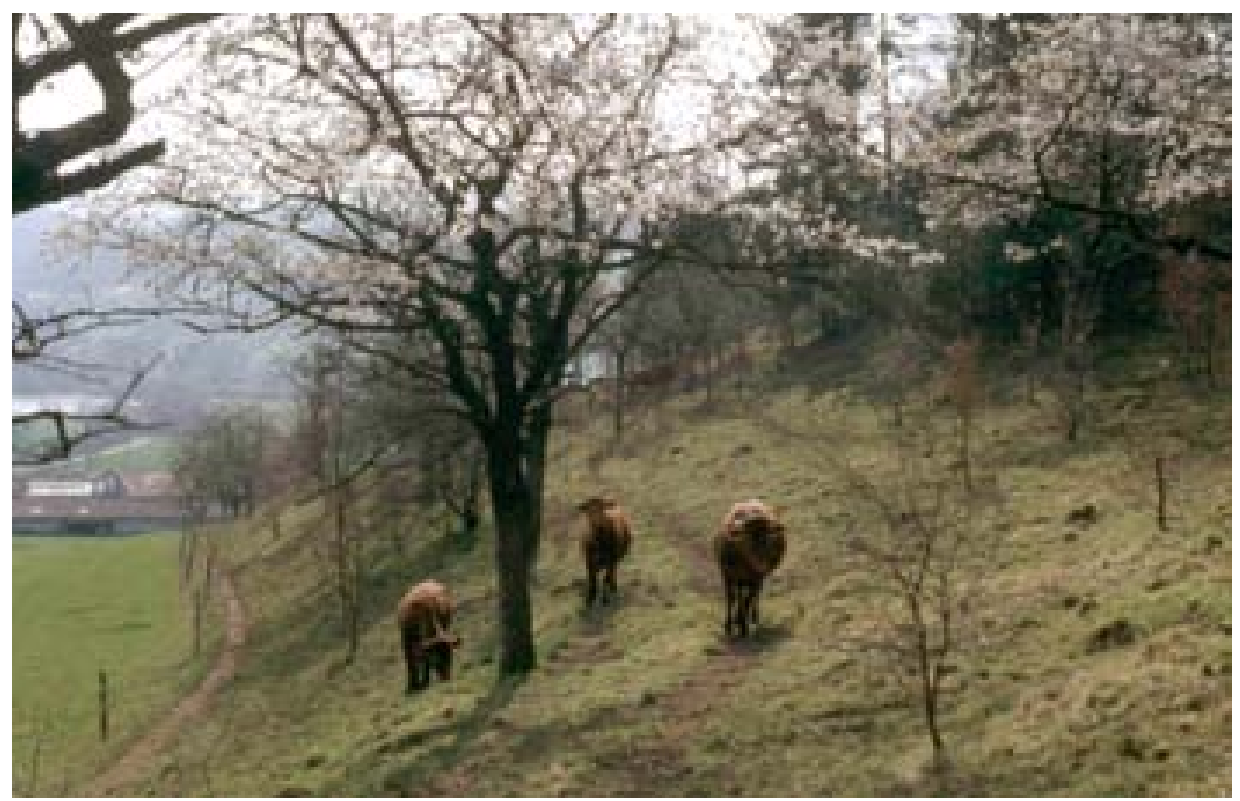

Abb. 61: Große Phytophage als große Ökosystemingenieure (Wiese 3).

Die Verbindung von ober- und unterirdischen Nahrungsnetzen ist nicht nur durch den Streufall gegeben, sondern durch die Wirkungen der Bodenbiota auf das Pflanzenwachstum und das ganze Nahrungsnetz sehr vielfältig. Collembolen können über eine Erhöhung der Stickstoffkonzentration in Gräsern die Blattlausentwicklung fördern (Scheu et al. 1999) aber auch als zusätzliche Nahrungsressource für generalistische Prädatoren in Zeiten geringer oberirdischer Phytophagendichte dienen (Scheu \& Setälä 2002).

Einen solchen Wechsel von Pflanzen-basierten zu Streu- bzw. Boden-basierten Nahrungsquellen konnte ich auch für die Ameisen durch die Analyse der stabilen Isotope bestätigen. Von April bis zum Juni sind die $\delta^{13} \mathrm{C}$-Werte der analysierten Ameisen angestiegen, im September näherten sie sich wieder den Werten der Pflanzen bei gleichzeitigem leichtem Absinken der ${ }^{15} \mathrm{~N}-$ Gehalte (Abb. 38, Kap. 6.3.1). Besonders deutlich war dieser Effekt bei Lasius flawns. Bodentiere hatten meist höhere ${ }^{13} \mathrm{C}$-Gehalte, was durch eine Anreicherung in verschiedenen Pilzen verursacht worden sein kann (Albers 2001). Während im Frühjahr noch nicht genügend Honigtau zur Verfügung steht und die Geschlechtstier-Larven einen hohen Proteinbedarf haben, scheinen die Ameisen verstärkt auf den Nahrungskanal des Streu-basierten Bodennahrungsnetzes zu wechseln; im Sommer wechseln dann viele Arten zu einer hauptsächlichen Honigtauernährung. Besonders Lasius alienus hat im Sommer sehr ähnliche Werte wie die oberirdischen Pflanzenteile. Myrmica sabuleti scheint sich immer noch zu einem etwas größeren Teil zoophag zu ernähren. Die Bodenbiota erhält nun aber gleichzeitig durch die Ameisen bessere Ressourcen bzw. Bedingungen, so dass sich im FreilandMikrokosmos-Versuch kein einheitlicher negativer Effekt auf die Springschwänze 
feststellen ließ. Die Blattläuse profitieren noch direkter von den Ameisen, da häufige Anwesenheit der Ameisen Parasitoide, andere Räuber und Konkurrenten abhält. Durch die Ameisen kann der positive Effekt der Collembola über die Pflanze auf die Blattläuse auch wieder positiv rückwirken, denn wenn die Ameisen zuckerhaltige Säfte in den Boden tragen, wachsen Pilze und Bakterien besser.

Die Auswirkungen der Ameisen auf das Pflanzenwachstum waren jedenfalls über die verbesserte Ressourcenverfügbarkeit (Kap. 8) wesentlich deutlicher als über die top-down-Wirkungen. Ebenso wirken die Ameisen auf die Bodenfauna offensichtlich eher über direkte Habitatveränderung oder verbesserte Nahrungsgrundlagen als über ihren räuberischen Effekt (Kap. 9). Prinzipiell gelten Saprophage als bottom-up-kontrolliert (Scheu \& Schaefer 1998), das scheint auch in Ameisennestern nicht anders zu sein.

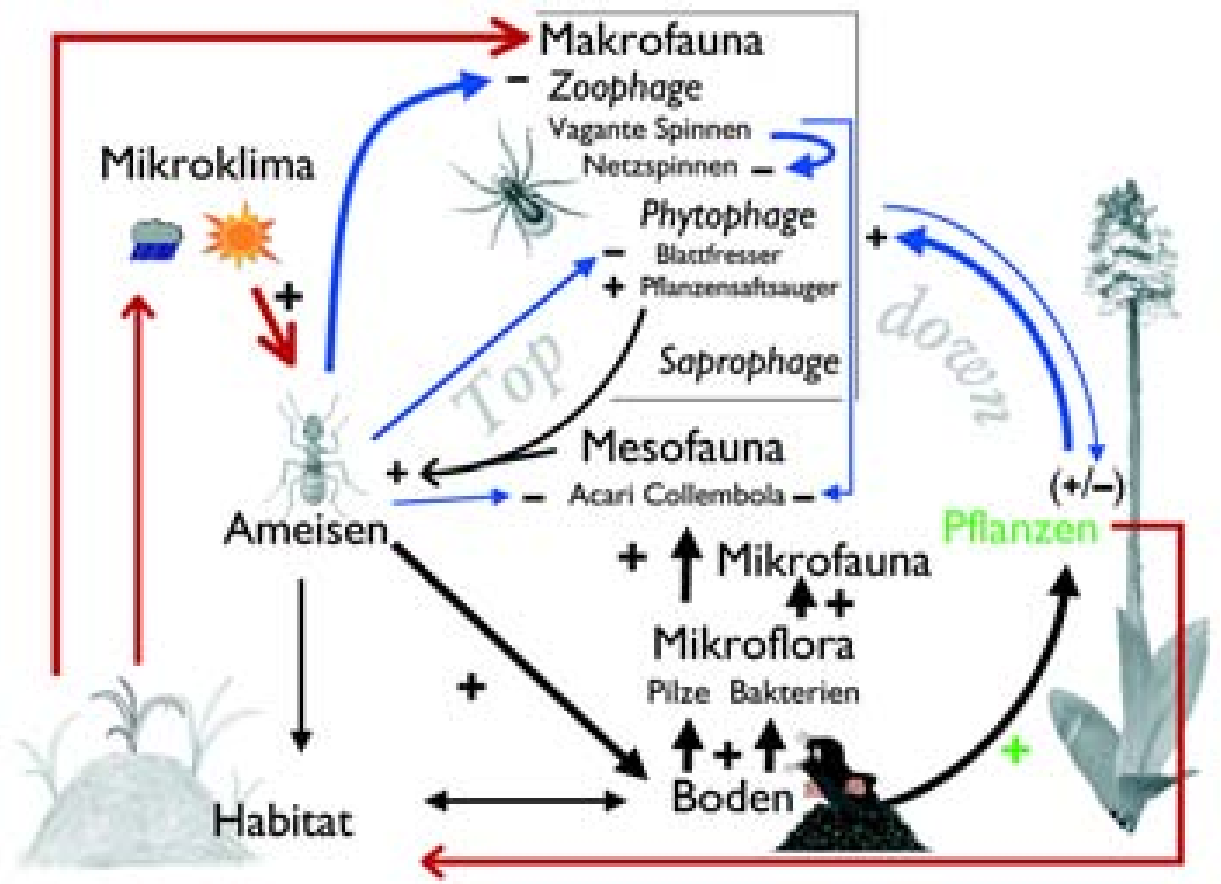

Abb. 62: Wechselwirkungen zwischen Ameisen, Pflanzen, Boden und Fauna, wie sie bei den Untersuchungen festgestellt und im Labor- und Freilandversuch bestätigt wurden.

Bottom-up Wirkungen im Nahrungsnetz lassen sich das ganze Jahr über nachweisen, im Frühjahr könnten dabei Nährstoffe, im Sommer die Wasserverfügbarkeit eine stärkere Rolle spielen. In der amerikanischen Prärie konnten Boyer et al. (2003) einen positiven Effekt der Beregnung im Sommer auch auf Phytophage feststellen, Spinnenreduktion hatte nur im Frühjahr und nur auf kauende Pflanzenfresser eine Auswirkung. Auf der Glatthaferwiese hingegen hatte Beregnung eher einen negativen Effekt auf die Phytophagen. Das Absammeln von Spinnen hatte auch im Sommer auf Blattkäfer einen leichten positiven Effekt. Auch die 
Tendenzen bei den Collembola und Auchenorrhyncha deuten auf eine top-downWirkung der Spinnen hin, wie sie auch für einige andere terrestrische Lebensräume beschrieben wurde (Halaj \& Wise 2001). Allerdings „tröpfeln“ die trophischen Kaskaden in terrestrischen Systemen eher. Intragildenprädation kann die topdown-Effekte ,verwischen“ (Wise et al. 1999, Snyder \& Wise 2001).

Auch im zweifaktoriellen Ausschlussversuch von Spinnen und Ameisen konnten keine deutlichen top-down Wirkungen sichtbar werden, weil durch die positive Entwicklung der Netzspinnen bei Ausschluss der vaganten Spinnen und Ameisen noch immer eine wichtige Prädatorengruppe in hohen Biomassen vorhanden war. Dadurch dass Ameisen und Spinnen Generalisten sind, entsteht ein hoher Grad an Redundanz. Allerdings sind komplexe Systeme stabiler (Polis 1998), so dass die einzelnen Taxa nicht funktionslos für den Erhalt der Ökosystemleistungen sind, wie ja gerade die drei Prädatorengilden zeigen: nur bei einer genügenden Populationsdichte und Artenvielfalt an Netzspinnen kann das Entfernen der beiden anderen Gruppen durch erhöhte Biomassezuwächse ausgeglichen werden. Im Unterschied $\mathrm{zu}$ manchen aquatischen Systemen kann also die top-downRegulierung nicht auf eine Schlüsselart reduziert betrachtet werden, sondern es gibt mehrere potentielle Schlüsselgruppen, die bei für sie günstigen Bedingungen diese Funktion übernehmen können. Im störungsfreien Zustand regulieren sich die Beutegreifer gegenseitig, so dass eine Übernutzung der teilweise gemeinsamen Ressourcen durch Selbstregulation verhindert wird.

Das Modellbild einer Kaskade ist anderseits insofern irreführend, weil die Stoffflüsse, anders als bei beispielsweise einer Hormonkaskade im Körper, immer von unteren trophischen Ebenen „,nach oben“ fließen - jedenfalls den möglichen top-down-Wirkungen entgegen. Für die steuernde Funktion im Ökosystem kann letztlich neben dem an Stoffflüsse gebundenen Nahrungsnetz ein Interaktionsnetz konstruiert werden, das neben den Stoff- und Energieflüssen auch Informationsflüsse (Schaefer 1996a) mit einbezieht. Jeder Organismus ist von seiner Nahrung gleichzeitig abhängig, so dass die regulierende Wirkung allein durch Entnahme dieser Ressourcen immer nur begrenzt sein kann. Viel stärker können steuernde Wirkungen durch den Aufbau oder die Veränderung von Strukturen sein, von denen der jeweilige Organismus selbst profitiert, da dann eine positive Rückkoppelung entsteht. Strukturen prägen Prozesse, Prozesse verändern Strukturen. Eine Steigerung der Zirkulation und ein ,gemeinsamer“ Aufbau von Strukturen zum gegenseitigen Nutzen auf Basis mehr oder weniger fester Symbiosen sind somit oft wichtigere Steuerungsgrößen als die Konkurrenz. 


\section{Zusammenfassung}

Ameisen können als boden- und streubewohnende eusoziale Prädatoren durch ihre zentrale Stellung im Nabrungsnetz das Gefüge der Interaktionen sowohl von der Basis her durch Modulation der Ressourcenverfügbarkeit ('bottom-up'-Wirkungen) als auch von der Spitze her durch Fraßdruck auf ihre Beutetiere ('top-down'Kontrolle) beeinflussen. Hügelbauende Arten können sich zu einem gewissen Maße von den Umweltbedingungen emanzipieren, indem sie sich eine günstigere Umgebung selbst gestalten; doch selbst solche Ökosystem-Ingenieure brauchen bestimmte mikroklimatische Bedingungen. Drei Bereiche dieser Rolle von Ameisen als Schlüsseltierarten im Grasland-Ökosystem wurden deskriptiv und experimentell untersucht:

\section{Umwelteinflïsse und Tiergemeinschaften}

Auf einem Halbtrockenrasen wurden die Verteilungsmuster der Ameisenarten und weiterer wichtiger Bodentiere in einem Gradientengefüge verschiedener Sukzessionsstufen und kleinräumiger Habitatvariationen in Abhängigkeit von den jeweiligen Umweltbedingungen raumbezogen erfasst und mit multivariaten Analyse- und Ordinationsverfahren ausgewertet. Auf einem xerothermen Hang auf Kalkstein im Werratal bei Witzenhausen wurde ein hexagonales $5 \mathrm{~m}$-Raster angelegt, das vom Buchen-Kiefern-Mischwald über Waldrand-, Jungwuchs- und Gebüschstrukturen bis auf einen Halbtrockenrasen reichte, der hangabwärts in eine trockene Glatthaferwiese mit altem Streuobstbestand und hainartigen Hecken übergeht. Die Makrofauna wurde überwiegend aus Bodensäulen ausgetrieben 
(Kempson) und aus den quantitativen Proben die Diversität und trophische Struktur der Tiergemeinschaft bestimmt (Kapitel 4).

Ameisen, Spinnen und Schnabelkerfe hatten ihre höchsten Dichten auf der Glatthaferwiese. Steinläufer, Schnurfüßer, saprophage Käferlarven und Pseudoskorpione waren hingegen in den Waldbereichen deutlich häufiger. Direkte Gradientenanalysen (RDA) und Kanonische Korrespondenzanalysen (CCA) konnten diesen Gradienten klar abbilden und dabei Hinweise auf die wesentlichsten strukturierenden Kräfte geben. Für die Ameisenarten waren die maximale Bodentemperatur und die Beschattung die wichtigsten verteilungsprägenden Umweltparameter - in den Bodenproben aus dem Wald dominierten beispielsweise Leptothorax-Arten, in denen aus der Wiese Myrmica sabuleti, Lasius alienus und Lasius flavus.

Der Einfluss des Mikroklimas auf die Ameisen und die Bodentiergemeinschaft wurde auf einer benachbarten Wiese durch experimentelle Manipulation der Faktoren Sonneneinstrahlung und Niederschlag untersucht. Mit transparenten Plexiglas-Dächern bzw. regendurchlässigen Schattendächern wurden Sonne und Regen zwei Jahre lang unabhängig voneinander reduziert und durch regelmäßiges Bewässern zusätzlicher Niederschlag simuliert (Kapitel 5).

Die Veränderungen des Mikroklimas hatten einen starken Einfluss auf die meisten Bodentiergruppen und veränderten kurz- und mittelfristig die physikalischen, chemischen und mikrobiologischen Charakteristika des Bodens. Ameisen und Schnabelkerfe hatten in den beschatteten Varianten deutlich niedrigere Dichten, für Spinnen und Weberknechte spielte die Habitatstruktur eine große Rolle: Weberknechte reagierten zunächst positiv auf die Beschattung, nahmen aber mittelfristig mit der schwindenden Vegetation in ihren Dichten deutlich ab. Saprophage waren auf den beregneten Parzellen signifikant häufiger, was mit einem deutlich stärkeren Streuschwund einherging. Die ober- und unterirdische Pflanzen-Biomasse nahm wie der Gesamt-Kohlenstoffgehalt des Bodens und die Diversität der Ameisen nach Beschattung sehr stark ab.

\section{Stellung und Funktion der Ameisen im Nahrungsnetz.}

Eine vergleichende Einordnung verschiedener dominanter Ameisen-, Spinnen und Zikadenarten in trophische Ebenen des Nahrungsnetzes eines Halbtrockenrasens ist durch Analyse der natürlichen Gehalte der stabilen Isotope ${ }^{13} \mathrm{C}$ und ${ }^{15} \mathrm{~N}$ möglich. Die Ermittlung der Stellung der Ameisen im Nahrungsnetz diente als Grundlage für die Untersuchung ihrer Funktion als Regulatoren von Pflanzenwachstum. Multitrophische Interaktionen wurden im Labor am Modellsystem Pflanze Pflanzenlaus - Ameise (Phaseolus vulgaris, Aphis fabae, Lasius niger) in neu entwickelten Mesokosmen untersucht (Kapitel 6).

Spinnen und Ameisen zeigten eine große Varianz und gegenseitige Überlappung ihrer ${ }^{15} \mathrm{~N} /{ }^{14} \mathrm{~N}-$ Verhältnisse. Lasius flavus und Lasius alienus hatten niedrigere $\delta$ ${ }_{15} \mathrm{~N}-$ Werte als Formica cunicularia, vermutlich wegen eines höheren Anteils an Trophobiose in ihrer Ernährung. Die vorwiegend räuberischen Arten Myrmica sabuleti und Myrmecina graminicola hatten das schwerere Isotop ${ }^{15} \mathrm{~N}$ stärker angerei- 
chert. Auch die Adulttiere der vaganten Spinnen hatten höhere ${ }^{15} \mathrm{~N} /{ }^{14} \mathrm{~N}$ Verhältnisse als die Jungtiere, was auf einen höheren trophischen Level hindeutet. Im Frühjahr schient die Bodenfauna eine wichtige Rolle als Nahrungsgrundlage für die Prädatoren zu spielen, Ameisen wechseln im Sommer verstärkt auf Honigtau der Blattläuse um; im Laborversuch hatte Zuckerzufütterung die deutlichsten positiven Auswirkungen auf die Ameisen.

Durch ein faktorielles Ausschlussexperiment von Ameisen und Spinnen wurde die Funktion wichtiger Prädatoren im Nahrungsnetz erforscht. In einem zweifaktoriellen Ausschlussversuch wurden die Dichten von vaganten Spinnen und Ameisen reduziert bzw. leicht erhöht. Die epi- und hypergäische Fauna wurde an drei Terminen von den eingezäunten Versuchsparzellen mit Saugfängen und Kempsonproben erfasst (Kapitel 7).

Vagante Spinnen und Ameisen reduzieren v.a. die Dichte der netzbauenden Spinnen, insbesondere die der Linyphiidae. Der negative Effekt der Ameisen auf die Dichte der Netzspinnen war zu den Hauptaktivitätszeiten im Juni und September am deutlichsten: Im Juni war in den Parzellen mit reduzierter Ameisenaktivität die Dichte der Fallenstellerinnen 2,5-fach und im September 3-fach so hoch wie in den Parzellen mit hoher Aktivität an epigäischen Ameisen. Neben der starken Intragilden-Interaktion wurden nur schwache top-down-Effekte nachgewiesen. Höhere Dichten an vaganten Spinnen senkten die Dichten der Zikaden und Springschwänze. Ortheziidae und Thysanoptera korrelierten stark positiv mit den Ameisendichten, was eine mutualistische Interaktion wahrscheinlich macht.

\section{Beeinflussung des Bodens und Wirkungen im Ökosystem}

Der bottom-up Einfluss von Ameisen durch Bodenveränderungen in der Rhizosphäre auf die Diversität der übrigen Bodenfauna und die Wuchsleistungen verschiedener Pflanzenarten wurde durch Untersuchung der biotischen und abiotischen Parameter und mit einem Mikrokosmosexperiment im Labor genauer analysiert. Der Boden aus Hügelnestern der Gelben Wiesenameise Lasius flavus wurde in einem faktoriellen Design im Frühjahr und Sommer an mehreren Terminen mit Boden von der umgebenden Wiese verglichen (Kapitel 8).

Die Nährstoffverhältnisse und die Bedingungen für die Mikroflora waren in den Nesthügeln signifikant günstiger als auf der umgebenden Wiese: die Gehalte an verfügbarem Stickstoff und Phosphor und Gesamt-Stickstoff waren wie die Mikrobielle Biomasse und Aktivität und die Dichte der Nematoden in den Nesthügeln deutlich erhöht. Der Wassergehalt schwankte in den Nesthügeln deutlich stärker, unterschied sich im Mittel aber, ebenso wie der $\mathrm{pH}-$ Wert, nicht von dem des Wiesenbodens.

Diese markanten Unterschiede führten auch in defaunierter Erde im Labor zu besserer Pflanzenentwicklung in Erde aus den Hügelnestern und stärkerem Nährstoffaustrag aus diesen Mikrokosmen. Dieser Effekt ließ sich an verschiedenen Pflanzen (Arrhenatherum elatius, Galium album, Trifolium repens) aus verschiedenen funktionellen Gruppen in fast allen faktoriellen Mischungen beobachten. 
Die Bedeutung der trophischen top-down Effekte über Herbivore in Relation zu den bottom-up Wirkungen durch „ecosystem engineering“ wurde durch ein Mikrokosmosexperiment im Freiland überprüft. Freiland-Mesokosmen mit Pflanzen verschiedener funktioneller Gruppen (Gras, Kräuter, Leguminose) und defaunierter Erde, die für Ameisen und andere Bodentiere durchlässig waren, wurden für ein Jahr innerhalb bzw. außerhalb von Nesthügeln von Lasius flavus inkubiert (Kapitel 9).

Die 100-fache Ameisendichte in den Nestern beeinflusste sowohl das Wurzelwachstum als auch die Struktur der Tiergemeinschaft deutlich: die Dichte von Spinnen und Oribatida war in den Kosmen in Nesthügeln signifikant niedriger, die Dichte aller Milben, der Pflanzenläuse und Schnellkäfer-Larven sowie die Mikrobielle Biomasse und das Trockengewicht der Wurzeln waren dort hingegen höher. Top-down-Effekte konnten nicht bestätigt werden, allerdings beeinflusst die Habitatmodifikation durch die Ameisen signifikant das Bodennahrungsnetz und damit auch wichtige Ökosystemprozesse wie die Stickstoffmineralisation.

Aus den Untersuchungen wird gefolgert (Kapitel 10), dass Ameisen eine wichtige Funktion als Ökosystemingenieure übernehmen und verglichen mit den deutlichen bottom-up-Wirkungen nur eine relativ schwache top-down-Kontrolle auf ihre Beutetiere ausüben. Neben dem Einfluss auf die Ressourcenverfügbarkeit für das ganze Nahrungsnetz beeinflussen Ameisen die übrigen Tiere direkt durch Interferenz und Störung und durch die Trophobiose mit Pflanzenläusen. Anhand des Ingenieursnetzes mit den Wechselwirkungen der Ameisen zu ihrer Umwelt wird deutlich, dass neben den trophischen Beziehungen Struktur-schaffende Wirkungen, besonders im Boden, mit ihren Interaktionen eine wesentliche Funktion in naturnahen terrestrischen Ökosystemen haben. 


\section{Literatur}

Albers, D. (2001): Nahrungsnetz und Stoffdynamik auf extensiv bewirtschafteten Ackerflächen - die Untersuchung stabiler Isotope $\left(\delta^{13} \mathrm{C}, \delta^{15} \mathrm{~N}\right)$ im ZersetzerSubsystem. Dissertation, Göttingen.

Alphei, J. (1995): Die freilebenden Nematoden von Buchenwäldern mit unterschiedlicher Humusform: Struktur der Gemeinschaften und Funktion in der Rhizosphäre der Krautvegetation. Dissertation, Göttingen (1994). (Berichte des Forschungszentrums Waldökosysteme Göttingen (A) 125: 1-165).

Alphei, J. \& H. Coenen (1999): Dachexperimente im Solling: Reaktion von Thekamöben und Fadenwürmern des Bodens. Allgemeine Forstzeitschrift 54: 64-68.

Anderson, J.P.E. \& K.H. Domsch (1978): A physiological method for the quantitative measurement of microbial biomass in soils. Soil Biology and Biochemistry 10: 215-221.

Andersen, A.N. \& A.D. Patel (1994): Meat ants as dominant members of australian ant communities - an experimental test of their influence on the foraging success and forager abundance of other species. Oecologia 98: 15-24.

Assing, V. (1986): Distribution, Densities and activity patterns of the ants (Hymenoptera: Formicidae) of Calluna Heathlands in Northwestern Germany. Entomologica Generalis 11: 183-190. 
Assing, V. (1994): Faunistische Notizen zur Ameisenfauna Südniedersachsens (Hymenoptera: Formicidae). Göttinger Naturkundliche Schriften 3: 33-40.

Bardgett, R.D. (2002): Causes and consequences of biological diversity in soil. Zoology 105: 367-374.

Bardgett, R.D., D.A. Wardle \& G.W. Yeates (1998): Linking above-ground and below-ground interactions: how plant responses to foliar herbivory influence soil organisms. Soil Biology and Biochemistry 30: 1867-1878.

Bauschmann, G. (1988): Faunistisch-ökologische Untersuchungen zur Kenntnis der Ameisen des Vogelsberges. Entomofauna 9: 69-115.

Beattie, A.J. (1989): The effects of ants on grasslands. Grassland structure and function: California annual grassland. Tasks for Vegetation Science 20: S. 105-116. Saarbrücken: Dellatinia.

Beck, T., R. G. Joergensen, E. Kandeler, F. Makeschin, E. Nuss, H. R. Oberholzer \& S. Scheu (1997): An inter-laboratory comparison of ten different ways of measuring soil microbial biomass C. Soil Biology and Biochemistry 29: 1023-1032.

Begon, M., J.L. Harper \& C.R. Townsend (1996): Ecology - Individuals, populations and communities. 3.Aufl. Oxford: Blackwell Science.

Blick, T., A. Hänggi \& K. Thaler (2002): Checkliste der Spinnentiere Deutschlands, der Schweiz, Österreichs Belgiens und der Niederlande (Arachnida: Araneae, Opiliones, Pseudoscorpiones, Scorpiones, Palpigradi). Version 1. Juni 2002. Internet: http://AraGes.de/checklisten.html

Blomqvist, M.M., H. Olff, M.B. Blaauw, T. Bongers \& W.H. van der Putten (2000): Interactions between above- and belowground biota: importance for small-scale vegetation mosaics in a grassland ecosystem. Oikos 90: 582-598.

Boyer, A.G., R.E. Swearingen, M.A. Blaha, C.T. Fortson, S.K. Gremillion, K.A. Osborn \& M.D. Moran (2003): Seasonal variation in top-down and bottomup processes in a grassland arthropod community. Oecologia 136: 309-316.

Brian, M.V. (1973): Temperature choice and its relevance to brood survival and caste determination in the ant Myrmica rubra L. Physiological Zoology 46: 245-252.

Brian, M.V. (1983): Social insects. London: Chapman and Hall.

Bristow, C.M. (1984): Differential benefits from ant attendance to two species of homoptera on New York ironweed. Journal of Animal Ecology 53: 715-726.

Brüning, A. (1991): The effect of a single colony of the red wood ant, Formica polyctena, on the spider fauna (Araneae) of a beech forest floor. Oecologia 86: 478-483.

Buckley, R.C. (1997a): Interactions involving Plants, Homoptera, and Ants. Annual Review of Ecology and Systematics 18: 11-135. 
Buckley, R.C. (1997b): Ant-Plant-Homopteran Interactions. Advances in Ecological Research 16: 53-85.

Carroll, C.R. \& D.H. Janzen (1973): Ecology of foraging by ants. Annual Review of Ecology and Systematics 4: 231-257.

Cherix, D. \& J.D. Bourne (1980): A field study on a super-colony of the Red Wood Ant Formica lugubris Zett. in relation to other predatory arthropodes (spiders, harvestmen and ants). Revue Suisse de Zoologie 87: 955-973.

Chu, H.F. (1949): The immature insects. Dubuque W.M. C. Brown Company Publishers

Clark, D.A. (2004): Tropical forests and global warming: slowing it down or speeding it up? Frontiers in Ecology and Environment 2: 73-80.

Closs, G.P., S.R. Balcombe \& M.J. Shirley (1999): Generalist Predators, Interaction Strength and Food-web Stability. Advances in Ecological Research 28: 93-126.

Crist, T.O. \& J.A. Wiens (1996): The distribution of ant colonies in a semiarid landscape - implications for community and ecosystem processes. Oikos 76: 301-311.

Curry, J.P. (1994): Grassland invertebrates : ecology, influence on soil fertility and effects on plant growth. London [u.a.] : Chapman \& Hall.

Cushman, J.H. \& J.F. Addicott (1989): Intra- and interspecific competition for mutualists: ants as a limited and limiting resource for aphids. Oecologia 79: 315-321.

Czerwiński, Z., H. Jakubczyk \& J. Pętal (1971): Influence of ant hills on the meadow soils. Pedobiologia 11: 277-285.

Czechowski, W. \& B. Pisarski (1992): Laboratory methods for rearing ants (Hymenoptera: Formicidae). Memorabilia Zoologica 45: 1-33.

Czechowski, W., A. Radschenko \& W. Czechowska (2002): The Ants (Hymenoptera, Formicidae) of Poland. Warszawa: Museum and Institute of Zoology PAS.

Dauber, J. \& V. Wolters (2000): Microbial activity and functional diversity in the mounds of three different ant species. Soil Biology and Biochemistry 32: 93 99.

de la Fuente, M.A.S. \& R.J. Marquis (1999): The role of ant-tended extrafloral nectaries in the protection and benefit of a Neotropical rainforest tree. Oecologia 118: 192-202.

Dean, W.R.J., S.J. Milton \& S. Klotz (1997): The role of ant nest-mounds in maintaining small-scale patchiness in dry grasslands in Central Germany. Biodiversity and Conservation 6: 1293-1307.

DeNiro, M.J. \& S. Epstein (1978): Influence of diet on the distribution of carbon isotopes in animals. Geochimica Et Cosmochimica Acta 42: 495-506. 
DeNiro, M.J. \& S. Epstein (1981): Influence of diet on the distribution of nitrogen isotopes in animals. Geochimica et Cosmochimica Acta 45: 341-351.

Djajakirana, G., R.G.Joergensen \& B. Meyer (1996): Ergosterol and microbial biomass relationship in soil. Biology and Fertility of Soils 22: 299-304.

Dlussky, G.M. (1981): Nester von Lasius flavus (Hymenoptera, Formicidae). Pedobiologia 21: 81-99.

Dumpert, K. (1978): Das Sozialleben der Ameisen. Berlin: Paul Parey.

Dunne, J.A., S.R. Saleska, M. Fischer \& J. Harte (2004): Integrating experimental and gradient methods in ecological cliate change research. Ecology 85: 904916.

Dyer, L.A. \& J.O. Stireman III (2003): Community-wide trophic cascades and other indirect interactions in an agricultural community. Basic and Applied Ecology 4: 423-432.

Elmes, G.W. (1974): The spatial distribution of a population of two ant species living in limestone grassland. Pedobiologia 14: 412-418.

Elmes, G.W. (1991): Ant colonies and environmental disturbance. Symposium of the Zoological Society of London 1991: 15-32. London.

Elmes, G.W. \& J.C. Wardlaw (1982): A population study of the ants Myrmica sabuleti and Myrmica scabrinodis, living at two sites in the south of England. I. A comparison of colony populations. Journal of Animal Ecology 51: 651-664.

Finke, D. \& R. Denno (2003): Intra-guild predation relaxes natural enemy impacts on herbivore populations. Ecological Entomology 28: 67-73.

Fischer, M.K., K.H. Hoffmann \& W. Völkl (2001): Competition for mutualists in ant-homopteran interaction mediated by hierarchies of ant attendance. Oikos 92: 531-541.

Fisher, B.L., L. da S.L. Sternberg \& D. Price (1990): Variation in the use of orchid extrafloral nectar by ants. Oecologia 83: 263-266.

Floren, A. \& S. Otto (2002): Beeinflusst die Anwesenheit der Waldameise Formica polyctena FOERSTER die Artenzusammensetzung und Struktur von Spinnengemeinschaften auf Eichen? Arachnologische Mitteilungen 24: 1-18.

Forster, J.R. (1778): Observations Made During a Voyage Round the World, on Physical Geography, Natural History, and Ethik Philosophy. G. Robinson, London.

Frampton, G.K., P.J. van den Brink \& P.J.L. Gould (2000): Effects of spring drougth and irrigation on farmland arthropods in southern Britain. Journal of Applied Ecology 37: 865-883.

Fraser, L.H. \& J.P. Grime (1998): Top-down control and its effect on the biomass and composition of three grasses at high and low soil fertility in outdoor microcosms. Oecologia 113: 239-246. 
Fricke, T. (2003): Agro-Climatic Data. Universität Witzenhausen, Department of Forage Production and Grassland Ecology. Internet: http://www.wiz.unikassel.de/pfb/klima/main.html

Fretwell, S.D. (1977): The regulation of plant communities by food chain exploiting them. Perspect. Biol. Med. 20: 169-185.

Frouz, J. (2000): The effect of nest moisture on daily temperature regime in the nests of Formica polyctena wood ants. Insectes Sociaux 47: 229-235

Gaye-Siessegger, J., U. Focken, S. Muetzel, H. Abel \& K. Becker (2004): Feeding level and individual metabolic rate affect $\delta^{13} \mathrm{C}$ and $\delta^{15} \mathrm{~N}$ values in carp: implications for food web studies. Oecologia 138: 175-183.

Gibb, H. \& D.F. Hochuli (2004): Removal experiment reveals limited effects of a behaviourally dominat species on ant assemblages. Ecology 85: 648-657.

Gösswald, K. (1990): Die Waldameise. Band 1 \& 2. Wiesbaden: Aula Verlag.

Graff, O. (1953): Die Regenwürmer Deutschlands. Forschungsanstalt für Landwirtschaft Braunschweig-Völkenrode Schriftenreihe Heft 7. Hannover: M. u. H. Schaper.

Gridina, T.I. (1990): Influence of Formica polyctena FOERST. (Hymenoptera, Formicidae) on the distribution of predatory arthropods in forest ecosystems. Memorabilia Zoologica 44: 21-36.

Gridina, T.I. (1994): Ethological aspects of interrelation between Formica polyctena FOERST. (Hymenoptera, Formicidae) and spiders and carabids. Memorabilia Zoologica 48: 91-97.

Gruner, H.E. (1966): Crustacea V: Isopoda, 2.Lieferung. In: DAHL, F. (Hrsg:): Die Tierwelt Deutschlands und der angrenzenden Meeresteile 53. Jena: Gustav Fischer Verlag

Halaj, J., D.W. Ross \& A.R. Moldenke (1997): Negative effects of ant foraging on spiders in Douglas-fir canopies. Oecologia 109: 313-322.

Halaj, J., D.W. Ross \& A.R. Moldenke (2000): Importance of habitat structure to the arthropod food-web in Douglas-fir canopies. Oikos 90: 139-152.

Halaj, J. \& D.H. Wise (2001): Terrestrial Trophic Cascades: How much do they trickle? The American Naturalist 157: 262-281.

Harte, J., A. Rawa \& V. Price (1996): Effects of manipulated soil microclimate on mesofaunal biomass and diversity. Soil Biology and Biochemistry 28: 313322.

Hawkins, B.A. (2004): Are we making progress towards understanding the global diversity?. Basic and Applied Ecology 5: 1-3

Heimer, S. \& W. Nentwig (1991): Spinnen Mitteleuropas. Berlin \& Hamburg: Paul Parey. 
Heitkamp, U. \& S. Schauermann (1982): Modifikationen zur Substratextraktion der Enchytraeidae mit einer Wassertauchmethode. Mitteilungen aus dem Sonderforschungsbereich 135: 33-38.

Hill, M.O. \& H.G. Gauch (1980): Detrended correspondence analysis, an improved ordination technique. Vegetatio 42: 47-58.

HLUG, (2003): Hessisches Landesamt für Umwelt und Geologie: Umweltatlas Hessen. Internet: http://atlas.umwelt.hessen.de/

HLVA (1996): Hessisches Landesvermessungsamt: Topographische Karte 1:50.000, Blatt 4724 „Witzenhausen“, Wiesbaden.

HVRKF (1999 \& 2003): Hessische Verwaltung für Kataster und Flurneuordnung: Luftbildvoransichten, Internet http://www.hkvv.hessen.de/produkte/luftbilder/index.htm

Hölldobler, B. \& C.J. Lumsden (1980): Territorial strategies in ants. Science 210: 732-739.

Hölldobler, B. \& E.O. Wilson (1990): The ants. Berlin: Springer.

Hölldobler, B. \& E.O. Wilson (1995): Ameisen - die Entdeckung einer faszinierenden Welt. Basel: Birkhäuser Verlag.

Holway, D.A., A.V. Suarez \& T.J. Case (1998): Loss of intraspecific aggression in the success of a widespread invasive social insect. Science 282: 949-952.

von Humboldt, A. (1808): Ansichten der Natur mit wissenschaftlichen Erläuterungen. J.G. Cotta, Tübingen, Germany.

Huxley, C.R. \& C.R. Cutler (1991): Ant-plant interactions. Oxford: Oxford University Press.

Jakubczyk, H., Z. Czerwiński \& J. Pętal (1972): Ants as agents of soil habitat changes. Ekologia Polska 20: 153-161.

Jeanne, R.L. (1979): A latitudinal gradient in rates of ant predation. Ecology 60: 1211-1224.

Joger, H.G. (1995): Untersuchungen zur epigäischen Fauna von Halbtrockenrasen: Anpassungen von Spinnen und Insekten an einen Extrem-Lebensraum. Dissertation, Göttingen.

Jones, C.G., J.H. Lawton \& M. Shachak (1994): Organisms as ecosystem engineers. Oikos 69: 373-386.

Kajak, A., A. Breymeyer \& J. Pêtal (1971): Productivity investigation of two types of meadows in the vistula valley. IX Predatory arthropods. Ekologia Polska 19: $223-233$.

Kajak, A., A. Breymeyer, J. Pêtal \& E. Olechowicz (1972): The influence of ants on the meadow invertebrates. Ekologia Polska 20: 163-171.

Karhu, K.J. (1998): Effects of ant exclusion during outbreaks of a defoliator and a sap-sucker on birch. Ecological Entomology 23: 185-194. 
Karhu, K.J. \& S. Neuvonen (1998): Wood ants and geometrid defoliator of birch: predation outweighs beneficial effects through the host plant. Oecologia 113: 509-516.

Kempson, D., M. Lloyd \& R. Ghelardi (1963): A new extractor for woodland litter. Pedobiologia 3: 1-21.

King, T.J. (1977a): The plant ecology of ant-hills in calcareous grasslands. I. Patterns of species in relation to ant-hills in southern England. Journal of Ecology 65: 235-256.

King, T.J. (1977b): The plant ecology of ant-hills in calcareous grasslands. II. Succession on the mounds. Journal of Ecology 65: 257-278.

King, T.J. (1977c): The plant ecology of ant-hills in calcareous grasslands. III. Factors affecting the population sizes of selected species. Journal of Ecology 65: 279-315.

King, T.J. (1981): Ant-hills and grassland history. Journal of Biogeography 8: 329334.

Kirchner, W. (1990): Die Erbeutung von Ameisen durch Netzspinnen. Zeitschrift für Angewandte Zoologie 77: 319-346.

Kneitz, G. (1970): Saisonale Veränderungen des Nestwärmehaushaltes bei Waldameisen in Abhängigkeit von der Konstitution und dem Verhalten der Arbeiterinnen als Beispiel vorteilhafter Anpassung eines Insektenstaates an das Jahreszeitenklima. Verhandlungen der Deutschen Zoologischen Gesellschaft, ed. W. Rathmeyer: S. 318-322. Stuttgart: Gustav Fischer.

Köhler, W., G. Schachtel \& P. Voleske, (2002): Biostatistik. Einführung in die Biometrie für Biologen und Agrarwissenschaftler, 3. Auflage, Berlin: Springer.

Krebs, C.J. (1985): Ecology. The experimental analysis of distribution and abundance, 3. Aufl. New York: Harper and Row.

Kronestedt, T. (1990): Seperation of two species standing as Alopecosa aculeata (Clerck) by morphological, behavioural and ecological characters, with remarks on related species in the pulverulenta group (Araneae, Lycosidae). Zoologica Scripta 19: 203-225.

Kutter, H. (1977): Hymenoptera: Formicidae. Insecta Helvetica, Fauna 6. Zürich: Schweizerische Entomologische Gesellschaft.

Kutter, H. (1978): Hymenoptera: Formicidae, Ergänzungsband. Insecta Helvetica, Fauna 6a. Zürich: Selbstverlag (Druck: Fotorotar AG).

Laakso, J. (1999): Short-term effects of wood ants (Formica aquilonia Yarr.) on soil animal community structure. Soil Biology and Biochemistry 31: 337-343.

Laakso, J. \& H. Setälä (1997): Nest mounds of red wood ants (Formica aquilonia): hot spots for litter-dwelling earthworms. Oecologia 111: 565-659. 
Laakso, J. \& H. Setälä (1998): Composition and trophic structure of detrital food web in ant nest mounds of Formica aquilonia and in the surrounding forest soil. Oikos 81: 266-278.

Laine, K.J. \& P. Niemelä (1980): The influence of ants on the survival of mountain birches during an Oporinia autumnata (Lep., Geometridae) outbreak. Oecologia 47: 39-42.

Lajtha, K. \& R.H. Michener (1994): Stable isotopes in ecology and environmental science. Oxford: Blackwell Science.

Letourneau, D.K. \& L.A. Dyer (1998): Experimental test in lowland tropical forest shows top-down effects through four trophic levels. Ecology 79: 1678-1687.

Lobry de Bruyn, L.A. \& A.J. Conacher (1994): The effect of ant biopores on water infiltration in soils in undisturbed bushland and in farmland in a semi-arid environment. Pedobiologia 38: 193-207.

Loeppert, R.H. \& D.L. Suarez (1996): Carbonate and Gypsum. In: Soil Science of America (Hrsg.): Methods of Soil Analysis - Part 3: Chemical Methods. SSSA Book Series 5: 437-474.

Ludwig, B., B. John, R. Ellerbrock, M. Kaiser \& H. Flessa (2003): Stabilization of carbon from maize in a sandy soil in a long-term experiment. European Journal of Soil Science 54: 117-126

MacFadyen, A. (1961): Improved funnel-type extractors for soil arthropods. Journal of Animal Ecology 30: 171-184.

Martens, J. (1978): Spinnentiere, Arachnida: Weberknechte, Opiliones. (Friedrich Dahl: Tierwelt Deutschlands 64). Jena: G. Fischer.

Messina, F.J. (1981): Plant protection as a consequence of an ant-membracid mutualism: interactions on goldenrod (Solidago sp.). Ecology 62: 1433-1440.

Mikola, J., G.M. Barker \& D.A. Wardle (2000): Linking above-ground and belowground effects in autotrophic microcosms: effects of shading and defoliation on plant and soil properties. Oikos 89: 577-587.

Minagawa, M. \& E. Wada (1984): Stepwise enrichment of $15-\mathrm{N}$ along food chains: Further evidence and the relation between $15-\mathrm{N}$ and animal age. Geochimica et Cosmochimica Acta 48: 1135-1140.

Morales, M.A. (2000): Survivorship of ant-tended membracid as a function of ant recruitment. Oikos 90: 469-476.

Neilson, R., D. Hamilton, J. Wishart, C.A. Marriott, B. Boag, L.L. Handley, C.M. Scrimgeour, J.W. McNicol \& D. Robinson (1998): Stable isotope natural abundances of soil, plants and soil invertebrates in an upland pasture. Soil Biology and Biochemistry 30: 1773-1782.

Nentwig, W., S. Bacher, C. Beierkuhnlein, R. Brandl \& G. Grabherr (2004): Ökologie. Heidelberg \& Berlin: Spektrum Akademischer Verlag. 
Nickel, H. \& R. Remane (2002): Artenliste der Zikaden Deutschlands, mit Angabe von Nährpflanzen, Nahrungsbreite, Lebenszyklus, Areal und Gefährdung. Beiträge zur Zikadenkunde 5: 27-64.

Nielsen, M.G., N. Skyberg \& L. Winther (1976): Studies on Lasius flavus F. (Hymenoptera, Formicidae): I. Population density, biomass, and distibution of nests. Entomologiske Meddelelser 44: 65-75.

Nkem, J.N., L.A. Lobry de Bruyn, C.D. Grant \& N.R. Hulugalle (2000): The impact of ant bioturbation and foraging activties on surrounding soil properties. Pedobiologia 44: 609-621.

Oelbermann, K. \& S. Scheu (2002): Stable isotope enrichment $\left(\delta^{15} \mathrm{~N}\right.$ and $\left.\delta^{13} \mathrm{C}\right)$ in a generalist predator (Pardosa lugubris, Araneae: Lycosidae): effects of prey quality. Oecologia 130: 337-344.

Offenberg, J. (2001): Balancing between mutualism and exploitation: the symbiotic interaction between Lasius ants and aphids. Behav. Ecol. Sociobiol. 49: 304-310.

Oksanen, L., S.D. Fretwell, J. Arruda \& P. Niemelä (1981): Exploitation ecosystems in gradients of primary productivity. The American Naturalist 118: 240 261.

Otto, D. (1967): Die Bedeutung der Formica-Völker für die Dezimierung der wichtigsten Schadinsekten - Ein Literaturbericht. Waldhygiene 7: 65-90.

Otto, D. (2003): Instinktmäßiges Verhalten und individuelle Eigenarten der Arbeiterinnen im Waldameisenvolk. Ameisenschutz aktuell 17: 65-73.

Paine, R.T. (1969): A note on trophic complexity and community stability. The American Naturalist 103: 91-93.

Perfecto, I. \& J. Vandermeer (1996): Microclimatic changes and the indirect loss of ant diversity in a tropical agroecosystem. Oecologia 108: 577-582.

Pętal, J. (1978): The role of ants in ecosystems. In: M.V. Brian (Hrsg.): Production ecology of ants and termites. Cambridge: Cambridge University Press.

Pętal, J. (1980): Ant populations, their regulation and effect on soil in meadows. Ekologia Polska 28: 297-326.

Pętal, J. (1981): Intraspecific competition as a way of adaptation to food resources in an ant population. Ekologia Polska 29: 421-430.

Pętal, J. (1998): The influence of ants on carbon and nitrogen mineralisation in drained fen soils. Applied Soil Ecology 9: 271-275.

Pętal, J., L. Andrzejewska, A. Breymeyer \& E. Olechowicz (1971): Productivity investigation of two types of meadows in the vistula valley $\mathrm{X}$ : The role of ants as predators in a habitat. Ekologia Polska 19: 213-222.

Pętal, J. \& A. Kusinska (1994): Fractional composition of organic matter in the soil of anthills and of the environment of meadows. Pedobiologia 38: 493501. 
Petelle, M., B. haines \& E. Haines (1979): Food preferences analysed using ${ }^{13} \mathrm{C} /{ }^{12} \mathrm{C}$ Ratios. Oecologia 38: 159-166.

Petersen, A. (1962): Larvae of insects: An introduction to Nearctic species. Parts I \& II. Ann Arbor: Edwards Brothers.

Peterson, B.J. \& B. Fry (1987): Stable isotopes in ecosystem studies. Annual Review of Ecology and Systematics 18: 293-320.

Pfister, V., B. Schäfer, A. Sührig \& A. Rothländer (2001): Diversity of spiders, carabid and staphylinid beetles in mixed and pure patches of beech and spruce: Small scale distribution patterns. In: G. Zotz \& C. Körner (Hrsg.): Funktionelle Bedeutung von Biodiversität. Verhandlungen der Gesellschaft für Ökologie 31: 164. Basel, Berlin: Parey.

Pimm, S., J. Lawton \& J. Cohen (1991): Food web patterns and their consequences. Nature 350: 669-674.

Pisarski, B. (1978): The role of ants in ecosystems - Comparison of various biomes. In: M.V. Brian (Hrsg.): Production ecology of ants and termites. Cambridge: Cambridge University Press.

Platen, R. (1985): Die Spinnentierfauna (Araneae, Opiliones) aus Boden- und Baumeklektoren des Staatswaldes Burgholz (MB 4708). Jahresberichte des Naturwissenschaftlichen Vereins Wuppertal 38: 75-86.

Platen, R., T. Blick, P. Bliss, R. Drogla, A. Malten, J. Martens, P. Sacher \& J. Wunderlich (1995): Verzeichnis der Spinnentiere (excl. Acarida) Deutschlands (Arachnida: Araneida, Opilionida, Pseudoscorpionida). Arachnologische Mitteilungen Sonderband 1, S. 1-55.

Platen, R., T. Blick, P. Sacher \& A. Malten (1998): Rote Liste der Webspinnen (Arachnida:Araneae). In: Rote Liste gefährdeter Tiere Deutschlands. Schriftenreihe für Landschaftspflege und Naturschutz 55: 268-275. Bonn: Bundesamt für Naturschutz.

Platner, C., S. Scheu \& M. Schaefer (1999): Kurzfristige Auswirkungen kleinräumiger Klimaveränderungen auf Ameisen (Formicidae) und andere Bodentiere einer Grünlandbrache. Mitteilungen der Deutschen Bodenkundlichen Gesellschaft 89: 281-284.

Platner, C., M. Schaefer \& S. Scheu (2001): Der Einfluss von Ameisen (Formicidae, Lasius flavus) auf den Boden und die mikrobielle Gemeinschaft trockener Brachwiesen. Mitteilungen der Deutschen Bodenkundlichen Gesellschaft 95: 84-87.

Platner, C., M. Schaefer \& S. Scheu (2001): Structural and functional relationships in pure and mixed stands of beech and spruce of different age: macrofauna community structure and litter decomposition. In: G. ZOTZ \& C. KÖRNER (Hrsg.): Funktionelle Bedeutung von Biodiversität. Verhandlungen der Gesellschaft für Ökologie 31: S. 157. Basel, Berlin: Parey. 
Polis, G.A. (1994): Food webs, trophic cascades and community structure. Australian Journal of Ecology 19: 121-136.

Polis, G.A. (1998): Stability is woven by complex webs. Nature 395: 744-755.

Polis, G.A. \& K.O. Winemiller (Hrsg.) (1996): Food webs: integration of patterns and dynamics. New York: Chapman \& Hall.

Ponsard, S. \& R. Arditi (2000): What can stable isotopes $\left(\delta^{15} \mathrm{~N}\right.$ and $\left.\delta^{13} \mathrm{C}\right)$ tell about the food web of soil macroinvertebrates. Ecology 81: 852-864.

Pontin, A.J. (1961): Population stabilization and competition between the ants Lasius flavus (F.) and L. niger (L.). Journal of Animal Ecology 30: 47-54.

Pontin, A.J. (1963): Further considerations of competition and the ecology of the ants Lasius flavus (F.) and L. niger (L.). Journal of Animal Ecology 32: 565574.

Pontin, A.J. (1978): The numbers and distribution of subterranean aphids and thier exploitation by the ant Lasius flavus (Fabr.). Ecological Entomology 3: 203-207.

Rachor, I. (2004): Nahrungsnetzanalyse und Freilandexperimente zum Einfluß von Ameisen und Spinne auf Phytophage eines Halbtrockenrasens. Diplomarbeit, Göttingen.

Reineking, A., R. Langel \& J. Schikowski (1993): 15-N, 13-C-on-line measurements with an elemental analyser (carlo erber, NA 1500), a modified trapping box and a gas isotope mass spectrometer (Finnigan, MAT 251). Isotopenpraxis Environmental Health Studies 29: 169-174.

Riechert, S.E. \& L. Bishop (1990): Prey control by an assemblage of generalist predators: spiders in garden test systems. Ecology 71: 1441-1450.

Rögener, J. \& J. Pfau (1994): Untersuchungen zur Ameisenfauna (Hym., Formicidae) eines Kalkmagerrasen-Gehölz-Komplexes des Halbesberges bei Witzenhausen (Werra-Meißner-Kreis). Braunschweiger Naturkundliche Schriften 4: 553-574.

Roberts, M.J. (1995): Spiders of Britain \& Northern Europe. Collins Field Guide. London: Harper Collins.

Rothländer, A., W. Entling \& M. Schaefer (2001): Diversity of epigeic arthropod communities in mixed and pure stands of beech and spruce I: Opilionida and Araneida. In: G. ZOTZ \& C. KÖRNER (Hrsg.): Funktionelle Bedeutung von Biodiversität. Verhandlungen der Gesellschaft für Ökologie 31: S. 158. Basel, Berlin: Parey.

Rothländer, A., Sührig, A., Entling, W., Schaefer, M. (2003): The effect of forest stand type on macrofauna abundance and diversity in old- and medium-aged mixed and pure stands of beech and spruce. In: Stadler J, I. Hensen, S. Klotz \& H. Feldmann (Hrsg.): Biodiversity - from patterns to processes. 
Verhandlungen der Gesellschaft für Ökologie 33: S. 365. Halle/Saale, Göttingen: Die Werkstatt.

Rosengren, R. (1986): Competition and coexitence in an insular ant community a manipulation experiment (Hymenoptera: Formicidae). Annales Zoologici Fennici 23: 297-302.

Rundel, P.W., J.R. Ehleringer \& K.A. Nagy (Hrsg.) (1989): Stable isotopes in ecological research. Ecological Studies 68. New York: Springer.

Sagers, C.L., S.M. Ginger \& R.D. Evans (2000): Carbon and nitrogen isotopes trace nutrient exchange in an ant-plant mutualism. Oecologia 123: 582-586.

Sakata, H. (1995): Density-Dependent Predation of the Ant Lasius niger (Hyenoptera: Formicidae) on Two Attended Aphids Lachnus tropicalis and Myzocallis kuricola (Homoptera: Aphididae). Researches on Population Ecology 37: 159-164.

Sakata, H. (1999): Indirect interactions between two aphid species in relation to ant attendance. Ecological Research 14: 329-340.

Sanders, D. (2004): Stellung und Funktion von Spinnen (Araneida) und Ameisen (Formicidae) im Nahrungsnetz eines Halbtrockenrasens - ein Freilandexperiment. Diplomarbeit, Göttingen.

Sankaran, M. \& D.J. Augustine (2004): Large herbivores suppress decomposer abundance in a semiarid grazing ecosystem. Ecology 85: 1052-1061.

Schaefer, M. (1990): The soil fauna of a beech forest on limestone: trophic structure and energy budget. Oecologia 82: 128-136.

Schaefer, M. (1991a): The animal community: diversity and resources. In: E. Röhrig \& B. Ulrich (Hrsg.): Temperate deciduous forests. S. 51-120. Amsterdam: Elsevier.

Schaefer, M. (1991b): Ecosystem processes: secondary production and decomposition. In: E. Röhrig \& B. Ulrich (Hrsg.): Temperate deciduous forests. S. 175-218. Amsterdam: Elsevier.

Schaefer, M. (1991c): Animals in European temperate deciduous forests. In: E. Röhrig \& B. Ulrich (Hrsg.): Temperate deciduous forests. S. 503-525. Amsterdam: Elsevier.

Schaefer, M. (1996a): Die Bodenfauna von Wäldern: Biodiversität in einem ökologischen System. (Akademie der Wissenschaften und der Literatur Mainz, Abhandlungen der Mathematisch-Naturwissenschaftlichen Klasse, Jahrgang 1996, Nr. 2). Stuttgart: Steiner.

Schaefer, M. (1996b): Die Artenzahl von Waldinsekten: Muster und mögliche Ursachen der Diversität. Mitteillungen der Deutschen Gesellschaft für Allgemeine und Angewandte Entomologie 10: 387-395.

Schaefer, M. (1996c): Diversity of the animal community in a beech forest - patterns and processes. In: Y. Steinberger (Hrsg.): Preservation of our world in 
the wake of change. Proceedings of the Sixth International Conference of the Israeli Society for Ecology \& Environmental Quality Sciences. Jerusalem, 1996. Jerusalem: ISEEQS Publication.

Schaefer, M. (1997a): Die Mannigfaltigkeit von Flora und Fauna - Zur ökologischen Bedeutung der Biodiversität. Jahrbuch der Akademie der Wissenschaften in Göttingen 1995: 58-79.

Schaefer, M. (1997b): The diversity of the fauna of two beech forests: some thoughts about possible mechanisms causing the observed patterns. In: A. Kratochwil (Hrsg.): Aspects of biodiversity in ecosystems - Analysis of different complexity levels in theorie and practice. S. 58-79. Dordrecht: Kluwer.

Schaefer, M. \& J. Schauermann (1990): The soil fauna of beech forests: comparison between a mull and a moder soil. Pedobiologia 34: 299-314.

Schaefer, M. (2000): BROHMER: Fauna von Deutschland. 20. Aufl., Wiebelsheim: Quelle \& Meyer.

Schaefer, M. (2002): The relationship between forest structure and animal species diversity. In: H. Spellmann (Hrsg.): Presentations of the $5^{\text {th }}$ International Workshop of the EU-LIFE-Projekt: „Demonstration of Methods to Monitor Sustainable Forestry". Göttingen: Cuvillier.

Schauermann, J. (1982): Verbesserte Extraktion der terrestrischen Bodenfauna im Vielfachgerät, modifiziert nach Kempson und MacFadyen. Kurzmitteilungen aus dem SFB135: Ökosysteme auf Kalkstein 1: 47-50.

Scheu, S. (1990): Die saprophage Makrofauna (Diplopoda, Isopoda und Lumbricidae) in Lebensräumen auf Kalkgestein: Sukzession und Stoffumsatz. Dissertation, Göttingen (1989) (Berichte des Forschungszentrums Waldökosysteme, Reihe A 57: 1-302).

Scheu, S. (1992): Automated measurement of the respiratory response of soil microcompartments: active microbial biomass in earthworm faeces. Soil Biology and Biochemistry 24: 1113-1118.

Scheu, S., D. Albers, J. Alphei, R. Buryn, U. Klages, S. Migge, C. Platner, J. Salamon (2003): The soil fauna community in pure and mixed stands of beech and spruce of different age: trophic structure and structuring forces. Oikos 101: $225-238$.

Scheu, S. \& M. Falca (2000): The soil food web of two beech forests (Fagus sylvatica) of contrasting humus form: stable isotope analysis of a macro- and mesofauna dominated community. Oecologia 123: 285-296.

Scheu, S. \& H. Setälä (2002): Multitrophic interactions in decomposer food-webs. In: T. Tschartke \& B.A. Hawkins (Hrsg.): Multitrophic Level Interactions. Cambridge: University Press. 
Scheu, S. \& M. Schaefer (1998): Bottom-up control of the soil macrofauna community in a beechwood on limestone: Manipulation of food resources. Ecology 79: 1573-1585.

Scheu, S., A. Theenhaus \& T.H. Jones (1999): Links between the detritivore and the herbivore system: effects of earthworms and collembolans on plant growth and aphid development. Oecologia 119: 541-551.

Schumacher, E. (2004): Untersuchungen zur Nahrungsbiologie von Ameisen im tritrophischen System Pflanze-Blattlaus-Ameise. Diplomarbeit, Universität Göttingen.

Seifert, B. (1982): Die Ameisenfauna (Hymenoptera, Formicidae) einer RasenWald-Catena im Leutratal bei Jena. Abhandlungen und Berichte des Naturkundemuseums Görlitz 56 (6): 1-18.

Seifert, B. (1983): The taxonomical and ecological status of Lasius myops Forel and first description of ist males. Abhandlungen und Berichte des Naturkundemuseums Görlitz 57 (6): 1-16.

Seifert, B. (1986): Vergleichende Untersuchungen zur Habitatwahl von Ameisen (Hymenoptera: Formicidae) im mittleren und südlichen Teil der DDR. Abhandlungen und Berichte des Naturkundemuseums Görlitz 59 (5): 1-124.

Seifert, B. (1988): A taxonomic revision of the Myrmica species of Europe, Asia Minor and Caucasia. Abhandlungen und Berichte des Naturkundemuseums Görlitz 62 (3): 1-75.

Seifert, B. (1991): The phenotypes of the Formica rufa complex in East Germany. Abhandlungen und Berichte des Naturkundemuseums Görlitz 65 (1): 1-27.

Seifert, B. (1993): Die freilebenden Ameisenarten Deutschland (Hymenoptera: Formicidae) und Angaben zu deren Taxonomie und Verbreitung. Abhandlungen und Berichte des Naturkundemuseums Görlitz 67 (3): 1-44.

Seifert, B. (1996): Ameisen: beobachten, bestimmen. Augsburg: NaturbuchVerlag.

Seifert, B. (1998): Rote Liste der Ameisen (Hymenoptera: Formicidae). In: Rote Liste gefährdeter Tiere Deutschlands. Schriftenreihe für Landschaftspflege und Naturschutz 55: 130-133. Bonn: Bundesamt für Naturschutz.

Sims, R.W. \& B.M. Gerard (1985): Earthworms. Synopsis of the British Fauna 31. London: Brill/Backhuys.

Snyder, W.E. \& D.H. Wise (2001): Contrasting trophic cascades generated by a community of generalist predators. Ecology 82: 1571-1583.

Sonnenburg, H. (1996): Die Ameisenfauna (Hymenoptera, Formicidae) von Streuobstwiesen bei Rastatt (Baden). Carolinea 54: 139-148.

Spedding, C.R.W. (1971): Grassland Ecology. London: Oxford University Press.

Stadler, B. \& A.F.G. Dixon (1998): Costs of ant attendance for aphids. Journal of Animal Ecology 67: 454-459. 
Stadler, B. (2001): Insektenökologie: Ein ökosystemarer Ansatz. Forstarchiv 72: 124-130.

Stadt Witzenhausen (2002): Flurstückkarte 1:1.000.

Stein, T.-M. (1996): Klimabeobachtungen in Witzenhausen für das Jahr 1995. Arbeiten und Berichte Nr. 43 des FG Kulturtechnik und Ressourcenschutz, FB 11, Universität-Gesamthochschule Kassel. Witzenhausen: Eigenverlag.

Steiner, M. \& B. C. Schlick-Steiner (2002): Einsatz von Ameisen in der naturschutzfachlichen Praxis. Naturschutz und Landschaftsplanung 34: 5-12.

Strauss, S.Y. (1987): Direct and indirect effects of host plant fertilisation on an insect community. Ecology 68: 1670-1678.

Sührig, A. (2004): Kurzflügelkäfer (Coleoptera: Staphylinidae) und Weberknechte (Arachnida: Opiliones) in Mischbeständen aus Fichte und Buche im Vergleich zu Fichten- und Buchenreinbeständen - eine Studie im Solling. Dissertation, Göttingen.

Sührig, A. \& M. Schaefer (2001): Diversity of epigeic arthropod communities in mixed and pure stands of beech and spruce II: Carabidae and Staphylinidae. In: G. Zotz \& C. Körner (Hrsg.): Funktionelle Bedeutung von Biodiversität. Verhandlungen der Gesellschaft für Ökologie 31: S. 159. Basel, Berlin: Parey.

Taraschweski, C., D. Sanders, H. Nickel \& C. Platner (2005): Effects of the waspspider, Argiope bruennichi, on plant- and leafhoppers. Beiträge zur Zikadenkunde 8: 49-58.

Tayasu, I., T. Abe, P. Eggleton \& D.E. Bignell (1997): Nitrogen and carbon isotope ratios in termites: an indicator of trophic habit along the gradient from wood-feeding to soil-feeding. Ecological Entomology 22: 343-351.

ter Braak, C.J.F. (1995): Ordination. In: R.H.G. Jongman, C.J.F. ter Braak \& O.F.R. van Tongeren (Hrsg.): Data Analysis in community and landscape ecology. S. 91-173. Cambridge, Wageningen: Cambridge University Press.

ter Braak, C.J.F. \& P. Smilauer (2002): CANOCO Reference manual and CanoDraw for Windows User's guide: Software for Canonical Community Ordination (vers. 4.5). Ithaca: Microcomputer Power.

Treseder, K.K., D.W. Davidson \& J.R. Ehleringer (1995): Absorption of antprovided carbon dioxide and nitrogen by a tropical epiphyte. Nature 375:137-139.

Vanderklift, M.A. \& S. Ponsard (2003): Sources of variation in consumer-diet $\delta^{15} \mathrm{~N}$ enrichment: a meta analysis. Oecologia 136: 169-182.

Völkl, W., J. Woodring, M. Fischer, M.W. Lorenz \& K.H. Hoffmann (1999): Antaphid mutualisms: the impact of honeydew production and honeydew sugar composition on ant preferences. Oecologia 118: 483-491. 
Wagner, D., M.J.F. Brown \& D.M. Gordon (1997): Harvester ant nests, soil biota and soil chemistry. Oecologia 112: 232-236.

Walker, M. \& T.H. Jones (2001): Relative roles of top-down and bottom-up forces in terrestrial tritrophic plant-insect herbivore-natural enemy systems. Oikos 93: $177-187$.

Waloff, N. \& R.E. Blackith (1962): The growth and distribution of the mounds of Lasius flavus (Fabricius) (Hym: Formicidae) in Silwood Park, Berkshire. Journal of Animal Ecology 31: 421-437.

Wardle, D.A. \& R.D. Bardgett (2004): Human-induced changes in large herbivorous mammal density: the consequences for decomposers. Frontiers in Ecology and the Environment 2: 145-153.

Way, M.J. (1963): Mutualism between ants and honeydew-producing homoptera. Annual Review of Entomology 8: 307-344.

Way, M.J. \& K.C. Khoo (1992): Role of ants in pest management. Annual Review of Entomology 37: 479-503.

Webb, N.R.a., S.J. Coulson, I.D. Hodkinson, W. Block, J.S. Bale \& A.T. Strathdee (1998): The effects of experimental temperature elevation on populations of cryptostigmatic mites in high Arctic soils. Pedobiologia 42: 298-308.

Wever, L.A., T.J. Lysyk \& M.J. Clapperton (2001): The influence of soil moisture and temperature on the survival, aestivation, growth and development of juvenile Aporrectodea tuberculata (Eisen) (Lumbricidae). Pedobiologia 45: 121133.

White, T.C.R. (1985): Green islands - nutrition not predation - an alternative hypothesis. Oecologia 67: 455-456.

White, T.C.R. (1998): Green islands still not explained. Oecologia 113: 517-518.

Whittaker, J.B. (1991): Effects of ants on temperate woodland trees. In: C.R. Huxley \& C.R. Cutler (Hrsg.): Ant-plant interactions. S. 67-79. Oxford: Oxford University Press.

Whittaker, J.B. \& N.P. Tribe (1998): Predicting numbers of an insect (Neophilaenus lineatus: Homoptera) in a changing climate. Journal of Animal Ecology 67: 987-991.

Wilkinson, L., G. Blank \& C. Gruber (1996): Desktop Data Analysis with Systat. New Jersey: Prentice Hall.

Wise, D.H. (1993): Spiders in ecological webs. Cambridge: Cambridge University Press.

Wise, D.H., W.E. Snyder \& P. Tuntibunpakul (1999): Spiders in decomposition food webs of agroecosystems: theory and evidence. Journal of Arachnology 27: 363-370.

Wolters, V. (1989): The influence of omnivorous elaterid larvae on the microbial carbon cycle in different soils. Oecologia 80: 405-413. 
Woltmann, L. (2002): Zur Wirkung von Sonneneinstrahlung und Niederschlag auf die Gemeinschaftsstruktur der Bodennematoden einer Glatthaferwiese - eine experimentelle Studie. Diplomarbeit, Göttingen.

Woodell, S.R.J. (1974): Ant-hill vegetation in a Norfolk salt marsh. Oecologia 16: 221-225.

Woodell, S.R.J. \& T.J. King (1991): The influence of mound-building ants on British lowland vegetation. In: C.R. Huxley \& C.R. Cutler (Hrsg.): Ant-plant interactions. S. 521-535. Oxford: Oxford University Press.

Yao, I., H. Shibao \& S. Akimoto (2000): Costs and benefits of ant attendance to the drepanosiphid aphid Tuberculatus quercicola. Oikos 89: 3-10.

Zoebelein, G. (1956a): Der Honigtau als Nahrung der Insekten. Teil I. Zeitschrift für Angewandte Entomologie 38: 369-416.

Zoebelein, G. (1956b): Der Honigtau als Nahrung der Insekten. Teil II. Zeitschrift für Angewandte Entomologie 39: 129-167. 


\section{Danksagung}

Mein herzlicher Dank gilt Prof. Dr. Matthias Schaefer für die Betreuung dieser Arbeit, die vielen Anregungen in interessanten Seminaren und Gesprächen, die Bereitstellung eines Arbeitsplatzes, die zahlreichen Möglichkeiten für mich, in Forschung und Lehre zu arbeiten und damit auch einen Teil dieser Arbeit zu finanzieren. Und nicht zuletzt für die akademischen Freiräume, die er den Mitarbeiterinnen und Mitarbeitern der Abteilung Ökologie gibt, wodurch wir in einem vielfältigen und harmonischen Arbeitsklima forschen konnten.

Prof. Dr. Stefan Scheu danke ich besonders für seine motivierende Einführung in die Bodenökologie, seine große Hilfe bei der Planung meiner Versuche und seinen stets hilfsbereiten, freundlichen, wertvollen und anregenden Rat.

Für die Übernahme des Korreferats danke ich Herrn Prof. Dr. Ulrich Ehlers.

Dr. Jürgen Schauermann stellte seine reichhaltige wissenschaftliche, praktische und organisatorische Erfahrung auch diesem Projekt hilfreich zur Verfügung.

Dirk Sanders, Evelyn Schumacher, Ingke Rachor und Lars Woltmann bereicherten das Projekt durch ihre engagierte Mitarbeit ungemein; Michael Sellner, Nicole Saverschek, Thomas Grützner, Stefan Dwillies, Anja Tittel und Acbim Rusteberg unterstützten uns durch kraftvolle Hilfe im Freiland, Gewächshaus und Labor.

Dr. Sonja Migge, Dr. Derk Albers und Alexander Sübrig danke ich besonders für das sehr gute Miteinander, die unterhaltsamen Kaffeerunden und den wissenschaftlichen Austausch im „Café Albers“. 
Judith Rothenbücher, Dr. Jörn Alphei, Axel Rothländer und Claus Döring standen bei Computer-Problemen jederzeit hilfreich zur Seite und halfen besonders durch ihre wichtigen Anregungen aus Statistik, Waldökosystem- und Biodiversitätsforschung.

Viele weitere wertvolle Hinweise erhielt ich von Prof. Dr. Alfred Buschinger, Dr. Jochen Gottwald, Dr. Jan Frour, Prof. Dr. Konrad Fiedler, Prof. Dr. David H. Wise und durch ihre besonders anregenden und hilfreichen Bücher auch von Dr. Bernhard Seifert, Prof. Dr. Edward O. Wilson und Prof. Dr. Bert Hölldobler.

Gudula Albert, Ingrid Kleinhans, Dieter Nünchert, Christel Fischer und Renate Grüneberg danke ich herzlich für ihre tatkräftige Hilfe in Labor und Werkstatt.

Danke an Dr. Herbert Nickel für die Zikadenbestimmung, Versorgung mit Brot und Wein und seine gelassene Grundeinstellung.

Familie Hain, Frau Waldheim, Herrn Müller und Herrn Graf von Berlepsch danke ich für die freundlichen Genehmigungen auf ihren Grundstücken (und Garagendächern) Freilandversuche vorzubereiten bzw. durchzuführen.

Für die massenspektrometrischen Analysen und Beratung danke ich Reinhard Langel, für die Ergosterol-Messungen Dr. Astrid Appubn und Prof. Dr. Rainer Jörgensen, für das Saatgut Rainer Bohnhorst und für die Blattläuse Dr. Bernd Ulber.

Mein besonderer Dank gebührt Geert, Margarete, Katharina und Angelika Platner für ihre Unterstützung und das Korrekturlesen.

Ganz besonders danke ich auch Ulrike Zander-Platner, Rosa Zander und Johanna Platner für ihre liebevolle Hilfe bei Tag und Nacht vom ersten Spatenstich bis zur letzten Seite.

Diese Arbeit wurde von der Deutschen Forschungsgemeinschaft gefördert, die durch ihre Sachbeihilfe das Projekt „Ameisen auf Halbtrockenrasen: Stellung im Nahrungsnetz, Einfluss auf Biodiversität im Boden und auf Pflanzenwachstum“ und damit einen Großteil meiner Arbeit ermöglichte.

Ich möchte auch allen Übrigen danken, die mich dabei unterstützt haben, diese Arbeit zu schreiben. Für mich war es eine sehr anregende, interessante und lehrreiche Zeit. 


\section{Anhang}

\section{A: Araneida}

Artenliste aller mit Bodenfallen, Bodenproben, Saugfängen und Handfängen gefangenen Spinnen.

\begin{tabular}{|c|c|}
\hline Atypidae & Atypus piceus (SULZER, 1776) \\
\hline Amaurobiidae & Callobius claustrarius (HAHN, 1833) \\
\hline Titanoecidae & Titanoeca quadriguttata (HAHN, 1833) (=T.obscura (WALCKENAER)) \\
\hline Dictynidae & Dictyna SUNDEVALL, 1833 \\
\hline Dysderidae & $\begin{array}{l}\text { Dysdera erythrina (WALCKENAER, 1802) } \\
\text { Harpactea lepida (C.L.KOCH, 1838) } \\
\text { Harpactea rubicunda (C.L.KOCH, 1838) }\end{array}$ \\
\hline Gnaphosidae & $\begin{array}{l}\text { Drassodes lapidosus (WALCKENAER, 1802) } \\
\text { Drassodes pubescens (THORELL, 1856) } \\
\text { Haplodrassus umbralitis (L. KOCH, 1866) } \\
\text { Zelotes aeneus (SIMON,1878) } \\
\text { Zelotes petrensis (C.L.KOCH, 1839) } \\
\text { Zelotes praeficus (L. KOCH 1866) } \\
\text { Zelotes subterraneus (C.L.KOCH, 1833) }\end{array}$ \\
\hline Clubionidae & Clubiona LATREILLE, 1804 \\
\hline Liocranidae & $\begin{array}{l}\text { Phrurolithus festivus (KOCH, 1835) } \\
\text { Scotina celans (BLACKWALL, 1841) }\end{array}$ \\
\hline Zoridae & Zora sylvestris KULCZYNSKI, 1897 \\
\hline $\begin{array}{l}\text { Heteropodi- } \\
\text { dae }\end{array}$ & Micrommata virescens (CLERCK, 1757) \\
\hline Thomisidae & $\begin{array}{l}\text { Osyptila atomaria (PANZER, 1801) } \\
\text { Xysticus kocbi THORELL, } 1872\end{array}$ \\
\hline $\begin{array}{l}\text { Philodromi- } \\
\text { dae }\end{array}$ & Tibellus oblongus (WALCKENAER, 1802) \\
\hline Salticidae & $\begin{array}{l}\text { Euophris aequipes (O.P.-CAMBRIDGE, 1871) } \\
\text { Euophris frontalis (WALCKENAER, 1802) } \\
\text { Evarcha falcata (CLERCK, 1757) (=E. flammata CLERCK) } \\
\text { Heliophanus dampfi SCHENKEL, 1923 } \\
\text { Heliophanus flavipes HAHN, 1832 } \\
\text { Myrmaracbne formicaria (DEGEER, 1778) } \\
\text { Neon reticulatus (BLACKWALL, 1853) } \\
\text { Pflegra fasciata (HAHN, 1826) } \\
\text { Pflegra fuscipes KULCZYNSK, 1891 } \\
\text { Salticus scenicus (CLERCK, 1797) }\end{array}$ \\
\hline
\end{tabular}




\begin{tabular}{|c|c|}
\hline Lycosidae & $\begin{array}{l}\text { Alopecosa taeniata (KOCH, 1835) } \\
\text { Alopecosa cuneata (CLERCK, 1757) } \\
\text { Alopecosa trabalis (CLERCK, 1757) } \\
\text { Arctosa lutetiana (SIMON, 1876) (= Triccia l. SIMON) } \\
\text { Arctosa personata L.KOCH, 1872 c.f. } \\
\text { Aulonia albimana (WALCKENAER, 1805) } \\
\text { Pardosa lugubris (WALCKENAER, 1802) } \\
\text { Pardosa palustris (LINNAEUS, 1758) } \\
\text { Trochosa terricola THORELL, } 1856\end{array}$ \\
\hline Pisauridae & Pisaura mirabilis (CLERCK, 1757) \\
\hline Agelenidae & $\begin{array}{l}\text { Coelotes terrestris (WIDER, 1834) } \\
\text { Coelotes inermis (L. KOCH, 1855) }\end{array}$ \\
\hline Hahnidae & Hahnia candida SIMON, 1875 \\
\hline Theridiidae & $\begin{array}{l}\text { Enoplognatha thoracica (HAHN) } \\
\text { Enoplognatha ovata (CLERCK) } \\
\text { Episinus angulatus (BLACKWALL) } \\
\text { Crustulina guttata (WIDER, 1834) } \\
\text { Theridion bimaculatum (LINNAEUS) }\end{array}$ \\
\hline $\begin{array}{l}\text { Tetragnathi- } \\
\text { dae }\end{array}$ & Tetragnatha extensa (LINNAEUS, 1758) \\
\hline Metidae & Metellina segmentata (CLERCK, 1757) \\
\hline Araneidae & $\begin{array}{l}\text { Araneus diadematus CLERCK, } 1757 \\
\text { Araniella cucurbitina (CLERCK, 1757) } \\
\text { Argiope bruennichi (SCOPOLI ,1772) } \\
\text { Mangora acalypha (WALCKENAER, 1802) }\end{array}$ \\
\hline Linyphiidae & $\begin{array}{l}\text { Centromerus sylvaticus (BLACKWALL, 1841) } \\
\text { Cnephalocotes obscurus (BLACKWALL) } \\
\text { Erigonella biemalis (BLACKWALL, 1841) } \\
\text { Gonatium rubellum (BLACKWALL, 1841) } \\
\text { Gonatium rubens (BLACKWALL, 1833) } \\
\text { Linyphia triangularis (CLERCK, 1757) } \\
\text { Meioneta affinis KULCZYNSKI, } 1898 \quad(=\quad \text { M. beata (O.P. } \\
\text { CAMBRIDGE)) } \\
\text { Meioneta rurestris (C.L.KOCH, 1836) } \\
\text { Minyriolus pusillus (WIDER, 1834) } \\
\text { Neriene peltata (WIDER, 1834) } \\
\text { Notioscopus sarcinatus (O.P. CAMBRIDGE, 1872) } \\
\text { Stemomyphantes lineatus (LINNAEUS, 1758) } \\
\text { Tenuiphantes flavipes (BLACKWALL, 1854) (=Lepthyphantes flavipes) } \\
\text { Tenuiphantes mengei KULCZYNSKI, 1887 (=Lepthyphantes mengei) } \\
\text { Tenuiphantes tenuis (BLACKWALL, 1852) (=Lepthyphantes tenuis) } \\
\text { Theonia cornix (SIMON, 1881) } \\
\text { Walckenaeria acuminata BLACKWALL, 1833 } \\
\text { Walckenaeria corniculans (O.P. CAMBRIDGE, 1875) }\end{array}$ \\
\hline
\end{tabular}




\section{B: Trockengewicht einiger Spinnenarten}

$\mathrm{w}=$ weiblich, $\mathrm{m}=$ männlich, repräsentative Einzeltiere und für Gattungen Mittelwerte aus allen Messungen. Aus SANDERS (2004)

\begin{tabular}{ll}
\hline & Gewicht in $\mathrm{mg}$ \\
\hline Atypus piceus & 117,00 \\
Trochosa terricola (w) & 26,61 \\
Alopecosa trabalis (m) & 21,70 \\
Xysticus kochi & 9,94 \\
Arctosa lutetiana (m) & 8,28 \\
Heliophanus dampfi (w) & 5,24 \\
Ozyptila atomaria & 4,26 \\
Atypus juv (Mittelwert) & 1,60 \\
Aulonia albimana (w) & 1,08 \\
Pflegra fuscipes (w) & 0,70 \\
Clubiona juvenil & 0,57 \\
Pardosa juvenil (Mittelwert) & 0,56 \\
Tenuiphantes (Mittelwert) & 0,31 \\
Linyphiinae (Mittelwert) & 0,22 \\
Walckenaeria acuminata (m) & 0,20 \\
Meioneta (Mittelwert) & 0,13 \\
Erigoninae (Mittelwert) & 0,11 \\
\hline
\end{tabular}

\section{C: Zikadenarten auf Wiese 1.}

Aus RACHOR (2004)

Deutscher Name

\section{Fulgoromorpha EVANS}

Cixiidae SPIN.

Glasflügelzikaden

(nur Larven)

Delphacidae LEACH

Asiraca clavicornis (F.)

Stenocranus minutus (F.)

Hyledelphax elegantula (BOH.)

Ribautodelphax pungens (RIB.)

\section{Cicadomorpha EvANs}

Cercopidae LEACH

Aphrophora alni (FALL.)
Spornzikaden

Schaufelspornzikade

Knaulgras-Spornzikade

Scheckenspornzikade

Zwenkenspornzikade

Schaumzikaden

Erlenschaumzikade 
Membracidae RAF.

Centrotus cornutus (L.)

Cicadellidae LATR.

Megophthalminae KIRK.

Megophthalmus scanicus (FALL.)

Agalliinae KIRK.

Anaceratagallia venosa (GEOFFR.)

Aphrodinae HPT.

Aphrodes bicincta (SCHRK.)

Anoscopus albifrons (L.)

Typhlocybinae KBM.

Emelyanoviana mollicula (BOH.)

Empoasca decipiens PAOLI

Eupteryx stachydearum (HARDY)

Eupteryx notata CURT.

Wagneripteryx germari (ZETT.)

Zygina byperici (H.-S.)

Arboridia parvula (BOH.)

Deltocephalinae FIEB.

Recilia coronifer (MARSH.)

Mocydia crocea (H.-S.)

Mocydiopsis attenuata (GERM.)

Streptanus marginatus (KBM.)

Adarrus multinotatus (BOH.)

Turrutus socialis (FL.)
Buckelzirpen

Dornzikade

Kleinzikaden

Kappenzikaden

Gemeine Kappenzikade

Dickkopfzikaden

Klee-Dickkopfzikade

Erdzikaden

Triftenerdzikade

Braune Erdzikade

Blattzikaden

Schwefelblattzikade

Gemüseblattzikade

Nördliche Ziestblattzikade

Triftenblattzikade

Kiefernblattzikade

Gemeine Johanniskrautzikade

Beilblattzikade

Zirpen

Kronengraszirpe

Safranzirpe

Westliche Märzzirpe

Schlängelschmielenzirpe

Gemeine Zwenkenzirpe

Triftengraszirpe 


\section{Lebenslauf}

Persönliche Daten:

Name: Christian Platner

Geburtsdatum: $\quad$ 09.02.1971

Geburtsort: Göttingen

Nationalität: deutsch

Familienstand: $\quad$ verheiratet seit $12.11 .1999,3$ Kinder

Schulbildung und Wissenschaftlicher Werdegang:

1977-1979 Grundschule Mittelpunktschule Hessisch-Lichtenau Walburg

1979-1983 Grundschule und Förderstufe Helfensteinschule in AhnatalWeimar

1983-1990 Gymnasium Wilhelmschule Kassel

$1990 \quad$ Abitur an der Wilhelmschule in Kassel.

1990-1997 Biologiestudium an der Georg-August-Universität Göttingen.

Titel der Diplomarbeit: „Untersuchungen zur Verteilung ausgewählter Gruppen der Makrofauna in einem Gradienten von Fichte (Picea abies) zu Buche (Fagus sylvatica) auf Buntsandstein“.

1992-2000 Chemiestudium an der Georg-August-Universität Göttingen.

Erste Staatsprüfung für das Lehramt an Gymnasien in den Fächern Biologie und Chemie.

1995-2001 Studentische und wissenschaftliche Hilfskraft am Institut für Zoologie, Universität Göttingen.

1999-2004 Promotion an der Abteilung Ökologie, Universität Göttingen, Professor Dr. M. Schaefer.

Titel der Doktorarbeit: „Ameisen als Schlüsseltiergruppe in einem Grasland - Studien zu ihrer Bedeutung für die Tiergemeinschaft, das Nahrungsnetz und das Ökosystem“.

2001-2004 Mitarbeiter im DFG-Projekt „Ameisen auf Halbtrockenrasen: Stellung im Nahrungsnetz, Einfluss auf Biodiversität im Boden und auf Pflanzenwachstum“.

Seit 2004 Wissenschaftlicher Mitarbeiter in der Abteilung Ökologie, Universität Göttingen 